\title{
Inelastic H-Atom scattering from ultra-thin films
}

\author{
Dissertation \\ zur Erlangung des mathematisch-naturwissenschaftlichen \\ Doktorgrades \\ "Doctor rerum naturalium" \\ der Georg-August-Universität Göttingen \\ im Promotionsprogramm Chemie \\ der Georg-August University School of Science (GAUSS)
}

\author{
vorgelegt von \\ Yvonne Jeannette Dorenkamp \\ aus Salzkotten
}

Göttingen, 2018 
Betreuungsausschuss:

Prof. Dr. Alec M. Wodtke, Dynamics at surfaces, Institut für Physikalische Chemie Göttingen/Max-Planck-Institut für Biophysikalische Chemie

Prof. Dr. Dirk Schwarzer, Reaction dynamics, Max-Planck-Institut für Biophysikalische Chemie

Dr. Oliver Bünermann, Atom-surface scattering dynamics, Institut für Physikalische Chemie Göttingen

Mitglieder der Prüfungskomission:

Referent: Prof. Dr. Alec M.Wodtke, Dynamics at surfaces, Institut für Physikalische Chemie Göttingen/Max-Planck-Institut für Biophysikalische Chemie

Korreferent: Prof. Dr. Dirk Schwarzer, Reaction dynamics, Max-Planck-Institut für Biophysikalische Chemie

Weitere Mitglieder der Prüfungskommission:

Prof. Dr. Jörg Behler, Theoretische Chemie, Institut für Physikalische Chemie Göttingen

Prof. Dr. Jörg Schroeder, Schroeder Group, Institut für Physikalische Chemie Göttingen

Prof. Dr. Jürgen Troe, Niedersachsen Research Professor, Institut für Physikalische Chemie Göttingen

PD Dr. Thomas Zeuch, Aggregation and Kinetics of Reactive Systems, Institut für Physikalische Chemie Göttingen

Tag der mündlichen Prüfung: 15.08.2018 


\section{Abstract}

This thesis contains experimental and theoretical fundamental studies of energy transfer processes on various surfaces via inelastic hydrogen atom scattering using the Rydberg atom tagging machine. The initial investigations of in total six late transition $f c c$-metals $(\mathrm{Au}, \mathrm{Pt}, \mathrm{Ag}, \mathrm{Pd}, \mathrm{Cu}, \mathrm{Ni}$ ) allowed to establish and optimize the experimental methodology as well as to simultaneously substantiate a theoretical model for the general description of energy-transfer processes on metal surfaces. Hence, it could be determined that the energy transfer for the investigated $f c c$-metals predominantly takes place via electron-hole-pair excitation. Despite its smaller contribution, phonon excitation was found to be essential in order to describe the experimentally determined (H/D)-isotope effects.

During the subsequently performed first hydrogen scattering experiments on insulating bulk as well as thin-layer surfaces, primarily direct inelastic scattering was observed that could be described by simple collision models. The variation of the layer thickness down to less than one monolayer of insulating aluminium oxide did not yield any significant changes of the scattering results, thus, a potential cooperative effect with the platinum substrate have been ruled out. These results as well as the scattering experiments performed on an oxygen covered $\mathrm{Pt}(111)$ surface would further suggest that scattering almost exclusively occurs on the quasi-isolated oxygen atoms on the surface.

In a concluding series of hydrogen scattering experiments of $(1 \times 1)$ oriented graphene and partially rotated graphene on $\mathrm{Ni}(111)$, it was possible to study the $\mathrm{C}-\mathrm{H}$ bond formation process between an impinging hydrogen atom and the graphene surfaces. The experimentally determined adsorption threshold significantly differs for both modification as well as with respect to prior studies of graphene on $\mathrm{Pt}(111)$, which could be explained by different interaction strengths between the graphene surface and the metal substrate. In addition, a detailed data analysis of the performed experiments further allowed to assign two new scattering components that previously had not been considered for graphene on $\operatorname{Pt}(111)$. 


\title{
List of abbreviations
}

\author{
AES Auger electron spectroscopy \\ AFM Atomic Force Microscopy \\ AIMD ab initio Molecular Dynamics \\ $\mathrm{AlO}_{\mathrm{x}} \quad$ Aluminum oxide \\ BBO Barium borate \\ BOA Born-Oppenheimer approximation \\ DFT Density Functional Theory \\ DOS Density of states \\ EDX Energy dispersive X-ray spectroscopy \\ EELS Electron energy loss spectroscopy \\ ehp Electron-hole-pair \\ EMT Effective Medium Theory \\ fcc $\quad$ Face centered cubic \\ FWHM Full width at half maximum \\ hcp Hexagonal closed packed \\ HRTEM High resolution transition electron microscopy \\ HRTOF $\mathrm{H}$ atom Rydberg tagging time-of-flight \\ KDP Potassium dideuterium phosphate \\ LEED Low-energy electron diffraction \\ MCP Micro-channel plate \\ MD Molecular Dynamics \\ Nd:YAG Neodymium-doped yttrium aluminium garnet \\ PES Potential energy surface \\ REMPI Resonance-enhanced multiphoton ionisation \\ RGA Residual gas analyser \\ TEM Transition electron microscopy \\ TOF Time-of-flight \\ UHV Ultra-high vacuum \\ UV Ultra-violet \\ VUV Vacuum-ultra-violet
}




\section{Contents}

$\begin{array}{lll}1 & \text { Introduction } & 1\end{array}$

\begin{tabular}{|lll}
2 & Fundamentals & 5
\end{tabular}

$2.1 \quad$ Scattering dynamics at surfaces $\ldots \ldots \ldots \ldots$. . . . . . . . . . . . . 5

$2.1 .1 \quad$ Simple collision models for energy transfer . . . . . . . . . . . . 6

$2.1 .2 \quad$ Born-Oppenheimer approximation (BOA) . . . . . . . . . . . . . 10

2.1.3 $\quad$ Molecular dynamics (MD) and ab initio molecular dynamics (AIMD) 12

$2.1 .4 \quad$ Failure of the Born-Oppenheimer approximation . . . . . . . . . 13

2.2 Relevant defined surfaces . . . . . . . . . . . . . . . . . . . . . . 16

2.2 .1 Metal surfaces . . . . . . . . . . . . . . . . . . . . . . 16

$2.2 .2 \quad$ Aluminum oxide surface . . . . . . . . . . . . . . . . . . . . 18

$2.2 .3 \quad$ Graphene surface . . . . . . . . . . . . . . . . . . . . . . . . . . . 19

\begin{tabular}{|lll}
\hline 3 & Experiment and applied methods & 27
\end{tabular}

3.1 Rydberg atom tagging surface scattering machine . . . . . . . . . . . . 28

3.2 Generation of well-defined $\mathrm{H} / \mathrm{D}$-atom beams . . . . . . . . . . . . . . . . . 29

3.3 Rydberg atom tagging time-of-flight $\ldots \ldots \ldots$. . . . . . . . . . . 34

3.4 Laser system . . . . . . . . . . . . . . . . . . . . . . . . . . . . . 36

$3.4 .1 \quad$ Laser set-up . . . . . . . . . . . . . . . . . . . . . . . . . . 37

3.4 .2 VUV generation . . . . . . . . . . . . . . . . . . . . . . 38

3.5 Experimental set-up, data acquisition and processing . . . . . . . . . . . . 40

3.6 Surface characterization techniques . . . . . . . . . . . . . . . . . 42

$3.6 .1 \quad$ Auger electron spectroscopy (AES) . . . . . . . . . . . . . . . . . 42

3.6 .2 Low energy electron diffraction (LEED) . . . . . . . . . . . . . . . 44

$3.7 \quad$ Surface preparation and characterization . . . . . . . . . . . . . . . . . 46

3.7 .1 Surface cleaning procedure. . . . . . . . . . . . . . . . . . 46

$3.7 .2 \quad$ Atomic oxygen covered $\mathrm{Pt}(111)$ surface . . . . . . . . . . . . . . . 48

$3.7 .3 \quad$ ALD prepared films $\ldots \ldots \ldots \ldots \ldots$. . . . . . . . . . . . 48

$3.7 .4 \quad$ Graphene on $\mathrm{Ni}(111) \ldots \ldots \ldots \ldots$. . . . . . . . . . . . 52 
$4 \quad \mathrm{H}$ and $\mathrm{D}$ atom scattering from transition metal surfaces 55

$4.1 \quad \mathrm{H}$ atoms scattering from $\mathrm{Au}(111)$ and insulating xenon surfaces . . . . . . 56

4.2 Dependence on experimental conditions _ . . . . . . . . . . . . . 59

4.3 Isotope effect in scattering experiment from $\mathrm{Au}(111)$ and $\mathrm{Ag}(111)$ surfaces 62

$4.4 \quad$ Scattering dynamics on six $f c c$ transition metal surfaces . . . . . . . . . . 65

4.4 .1 Experimental results . . . . . . . . . . . . . . 65

$4.4 .2 \quad$ Comparison to theoretical calculations . . . . . . . . . . . . 69

4.5 Discussion $\ldots \ldots \ldots \ldots \ldots$

\begin{tabular}{|lll}
5 & Ultra Thin Oxide Layer & 77
\end{tabular}

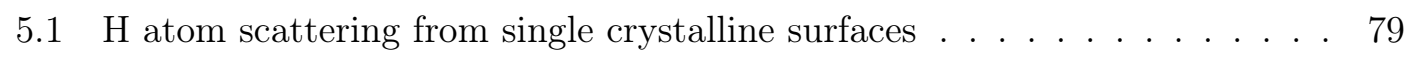

$5.2 \quad \mathrm{H}$ tom scattering from ALD prepared thin layer $\mathrm{AlO}_{\mathrm{x}}$ surfaces . . . . . . . 86

$5.3 \quad \mathrm{H}$ atom scattering from oxide monolayer on $\mathrm{Pt}(111) \ldots \ldots$

5.4 Discussion $\ldots \ldots \ldots \ldots \ldots \ldots \ldots$

\begin{tabular}{lll}
\hline 6 & Graphene on $\mathrm{Ni(111)}$ & 97
\end{tabular}

6.1 Previous studies of Graphene on $\mathrm{Pt}(111)$ and theoretical calculations . . . 99

$6.2 \quad \mathrm{H}$ atom scattering from Graphene on $\mathrm{Ni}(111)$. . . . . . . . . . . . . . 105

6.2.1 Comparison between (1 x 1) Graphene on $\mathrm{Ni}(111)$ and pristine

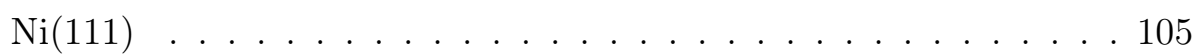

6.2 .2 2D Transaltional energy and angular distribution spectra of Graphene on $\mathrm{Ni}(111)$. . . . . . . . . . . . . . . . . . . . . . 107

6.3 Quasi-elastic scattering component . . . . . . . . . . . . . . . . . . . 111

6.4 Comparison between Graphene on $\mathrm{Ni}(111)$ and Graphene on $\operatorname{Pt}(111)$. . . 112

6.5 Comparison between (1 x 1) oriented and rotated Graphene on Ni(111). . 114

6.6 Scattering dynamics at different azimuthal orientations . . . . . . . . . . 116

6.7 Adsorption threshold determination . . . . . . . . . . . . . . . . 118

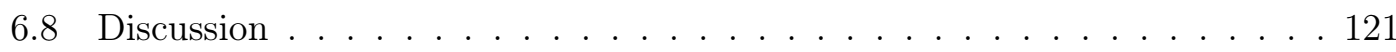

\begin{tabular}{lll}
\hline & Conclusion and Outlook & 123
\end{tabular}

\begin{tabular}{ll}
\hline Appendix & 127
\end{tabular}

\begin{tabular}{ll}
\hline Bibliography & 151
\end{tabular}

\begin{tabular}{ll}
\hline Acknowledgement & 165
\end{tabular} 


\section{Introduction}

To this day atomic and molecular processes on surfaces with their various industrial applications have essentially shaped history of mankind. On one hand, the invention of the Haber-Bosch process for the artificial ammonia synthesis 1 enabled the tremendously high human population growth to currently around 7.5 billion people during the last century. 2 On the other hand, hydrogen storage 3 and heterogeneous catalyzed processes 4 are believed to play a key role in solving the global energy problem and would thereby highly contribute to a sustainable future development of mankind. Furthermore, surface chemistry currently plays a crucial role for the industrial production of pharmaceutical, fertilizer and chemicals used in our daily life (e.g. Fischer-Tropsch), in automobile catalysts, micro- and nanotechnological applications (airbags, smart-phones), and in atmospheric and interstellar chemistry. 4

After the initial discovery and subsequently industrial application of prominent heterogeneous processes, scientists have made tremendous efforts to gain a deeper understanding of the underlying chemical and physical dynamics, which consequently allowed a continuous improvement of these processes. Moreover, the significance of the field of surface chemistry is widely recognized in the scientific community what clearly becomes apparent by the high number of Nobel prize laureates (prominent examples in Table 1.1) that dedicated themselves to the understanding of the fundamentals of surface science. 
Table 1.1: Selected list of Noble prize laureates in surface chemistry. 5 Nobel price laureate Year Contributions

\begin{tabular}{|c|c|c|}
\hline P. Sabatier & 1912 & $\begin{array}{l}\text { "for his method of hydrogenating organic com- } \\
\text { pounds in the presence of finely disintegrated } \\
\text { metals whereby the progress of organic chemistry } \\
\text { has been greatly advanced in recent years" }\end{array}$ \\
\hline F. Haber & 1918 & "for the synthesis of ammonia from its elements" \\
\hline I. Langmuir & 1932 & $\begin{array}{l}\text { "for his discoveries and investigations in surface } \\
\text { chemistry" }\end{array}$ \\
\hline $\begin{array}{l}\text { C. N. Hinshelwood, } \\
\text { N. N. Semenov }\end{array}$ & 1956 & $\begin{array}{l}\text { "for their researches into the mechanism of } \\
\text { chemical reactions" }\end{array}$ \\
\hline $\begin{array}{l}\text { D. R. Herschbach, } \\
\text { Y. T. Lee, J. C. Polanyi }\end{array}$ & 1986 & $\begin{array}{l}\text { "for their contributions concerning the dynamics } \\
\text { of chemical elementary processes" }\end{array}$ \\
\hline G. Ertl & 2007 & $\begin{array}{l}\text { "for his studies of chemical processes on solid } \\
\text { surfaces" }\end{array}$ \\
\hline
\end{tabular}

Since the foundation of surface chemistry by Sabatier and Haber at the beginning of the $20^{\text {th }}$ century, fundamental understanding of surface reactivity was subsequently established by Langmuir, Hinshelwood, and many more. The next breakthrough of this field came in the 1960s, when Ertl and others started to understand the dynamics of surface reactions on a truly atomic and molecular level.4 4

Notwithstanding the rapid developement of this field, until today still little is known about the details of energy exchange processes which are involved in gas-surface interactions, which play a crucial role in heterogeneous reactions as such interactions influence adsorption, diffusion, reaction and desorption dynamics. Understanding of these fundamental surface processes and how they affect reaction rates and selectivity can in principle lead to further improvements and better design of technology. 6

Atomic and molecular beam scattering experiments combined with laser state resolved studies are powerful tools to gain deeper insights into the underlying dynamics by investigating energy transfer processes between the atomic and molecular translational and internal degrees of freedom and the phononic and electronic degrees of freedom of the surface states. 6 6 7 .

One particular area of interest is the study of the role of energy transfer from small molecules or atoms to surfaces during the adsorption process. While at first the efficiency 
of the energy transfer (in particular the translational energy) highly affect the trapping probability of atoms or small molecules it is also of interest to understand how the transferred energy is dissipated among the energy degrees of freedom of the surface. Simplified experimental models can act as benchmark systems in order to partially explain the different energy transfer channels and to draw comparisons to theoretical predictions.

Well-defined single crystal surfaces are often used as model systems as their surface properties are well known and mathematically describable, which allows for targeted studying of the influence of various material properties in gas-surface interactions.

As simple model systems transition metal surfaces are frequently studied, not only due to their commercial availability and thermal stability but also due to their relevance in various heterogeneous catalytic processes. 6

While such simple systems are ideal for the validation of new experimental techniques and can act as benchmark for theoretical models, another growing area in the field of surface science are the artificial design of new types of surfaces such as nanostructures. For instance, thin film materials with nanoscale thickness have gained great attention in the last few decades as they can exhibit physical properties which strongly differ from the bulk material. In the literatue to date, a variety of examples have been reported in which electrical, mechanical, optical, or thermal material properties can be changed by altering solely the film thickness. $\frac{8}{6}$ For example, investigations of multilayer films of dissimilar materials such as $\mathrm{W} / \mathrm{ZrO}_{2}$ or $\mathrm{W} / \mathrm{Al}_{2} \mathrm{O}_{3}$ showed that thermal conductivity can be drastically lowered as soon as the layer thickness is reduced below the phonon mean free path.910 Other studies revealed strong effects of the electronic structure of metal-insulator-metal films with regards to the film thickness.11 12 Despite the high number of reported studies of thin film materials to the best of knowledge there have been no studies on energy transfer processes for such surfaces.

Within the area of ultra thin film materials, the atomically thin layered structure graphene represents a unique case. Graphene consists of a two-dimensional honeycomb layer of carbon atoms and stands out due to its electronic properties (extremely high electron mobility) 13 and extraordinary mechanical stability. 14 In addition, graphene is believed to play a key role in the interestellar $\mathrm{H}_{2}$ formation, 15 16 and is frequently discussed in the context of hydrogen storage, $17 \sqrt{18}$ nuclear fusion technology 19 and in electronic and spintronic devices. $20 \mid 21] 22$ It should further be noted that Geim and Novoselov were honored with the Nobel prize in physics in 20105 for the first production of free-standing graphene of micrometer dimensions. 23

Taking graphene as one of the most recent and prominent examples, it demonstrates that 
despite its long history, there still remains much to explore within the field of surface science.

Hydrogen atom scattering from well-defined surfaces in ultra-high vacuum (UHV) is one of the simplest interactions one may think of as in hydrogen atom-surface collision only the atomic translational energy can couple to the surface energy degrees of freedom, no rotations, vibrations or steric effects need to be considered. This makes it in particular attractive to investigate and compare to first principle theories. Furthermore, interaction processes of hydrogen atoms with solid surfaces are highly relevant in many technological applications, e.g. heterogeneous catalysis, 24 in interstellar $\mathrm{H}_{2}$ production on dust gains, 25 nuclear fusion in tokamaks or hydrogen storage $26 \sqrt{27}$

In this thesis, experiments were performed with the recently developed Rydberg atom tagging (RAT) machine.28 The RAT-machine generates a monoenergetic hydrogen atom beam with tunable energy and detects angular resolved scattered hydrogen atoms by high resolution time-of-flight (HR-TOF).

Using this sophisticated experimental set-up, at first simple single crystal transition metal surfaces of the $\mathrm{Cu}$ and $\mathrm{Ni}$ group will be investigated in order to establish a basic understanding of the experimental processes and in order to validate the theoretical models that were applied throughout this work. The methodology will subsequently extended to insulating surfaces such as $\mathrm{Al}_{2} \mathrm{O}_{3}$ as well as ultra thin films of alumina on a platinum substrate. Finally, inspired by the previous studies of free standing graphene on Pt(111) by Hongyang Jiang,29 the last chapter will deal with hydrogen scattering experiments performed on an atomically thin graphene layer on $\mathrm{Ni}(111)$. This chapter will answer the question of how the different nature of a metal surface influences the reactivity of the adsorbed graphene substrate. 


\section{Fundamentals}

\subsection{Scattering dynamics at surfaces}

When an atom collides with a surface there are three principle interaction mechanisms of how the atom can leave the surface: elastic scattering, inelastic scattering or trappingdesorption.

Elastic scattering means that no measurable amount of energy is transferred between the impinging atom and the surface during the collision process and the scattered atoms therefore retain their initial energy. In a direct inelastic scattering process, the atoms interact with the surface by a single encounter and exchange energy with the surface modes. In the aforementioned two cases, the information about the incidence conditions of the atoms is mostly preserved. In contrast, when the atom bounce off twice or more from the surface or even penetrate into the surface until its trajectory points again away from the surface, it is believed that an atom should lose most of its initial information. During each encounter, energy is transferred between the atom and the surface.

Trapping-desorption occurs, when a particle approaches the surface and loses sufficient translational energy to the degrees of freedom of the surface to be trapped on the surface. In this case, the particle remains long enough on the surface to equilibrate thermally with the surface and to lose all information about the incidence conditions. Subsequently, the particle desorbs with a translational energy distribution which can be described by a Maxwell-Boltzmann distribution determined by the surface temperature or effetive desorption temperature. 30

Complete theoretical descriptions of energy exchange processes between the impinging gas particles and surfaces are rather complex, involving both electronic and nuclear transitions as well as rearrangements. Despite enormous advances in computational power during the last decades, modeling of gas-surface interactions still remains a challenge today. 31 Especially for light molecules and atoms as well as for electronic excitation, quantum effects need to be considered. However, if the energy transfers in gas-surface interaction proceeds mainly on an adiabatic level (electronic inelastic effects are neglected) the dynamics might be described classically within the Born-Oppenheimer approximation. $32 \mid 33$ 
In the non-adiabatic case, the Born-Oppenheimer approximation is no longer valid and more sophisticated theoretical concepts are required.

Nevertheless, simple models can deepen the understanding of the physical dynamics even though the numerical agreement between the simulation and experiment is frequently not perfect. 31

\subsubsection{Simple collision models for energy transfer}

A simple mechanical model, which considers the interaction of an incoming atom with a surface as a single hard cube collision, 34 frequently is sufficient in order to achieve a good agreement with the experimetal data when direct scattering events are dominant (Figure 2.1 a)).6[31

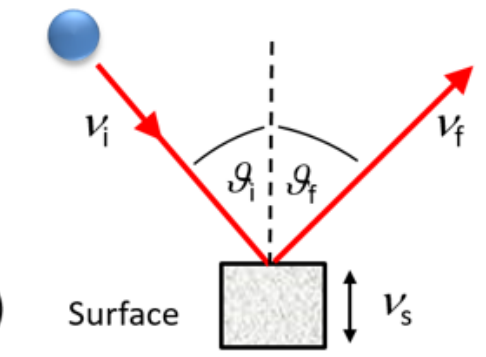

b)

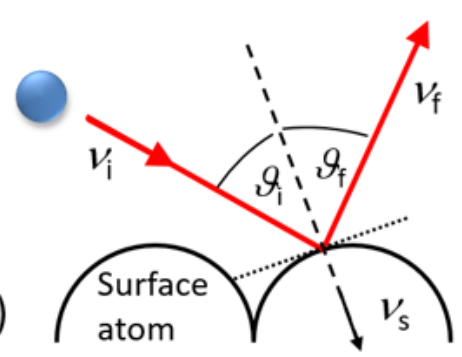

Figure 2.1: a) Binary collision between an atom and a surface represented as a hard cube. b) Atom scattering from individual surface atoms.

The hard cube model implies the following assumptions:

- The surface is considered to be perfectly smooth.

- Parallel momentum of the atom with respect to the surface plane is conserved in the course of the collision process.

- Changes of the atom normal momentum with respect to the surface plane are determined by conservation of energy and normal momentum.

- The surface "cube" is freely moving with a Maxwellian initial normal momenta distribution at the surface temperature $T_{\mathrm{s}}$.

The incoming atom (blue circle) with the velocity $\nu_{\mathrm{i}}$ hits the surface at an incidence angle $\vartheta_{\mathrm{i}}$ with respect to the surface normal (dashed line) and rebounds from the surface with the velocity $\nu_{\mathrm{f}}$ at an angle $\vartheta_{\mathrm{f}}$. 
In a first approximation, the collision is assumed to be purely repulsive and the surface cube sits initially at rest. Conservation of momentum and energy (2.1-2.3) yields the translational energy $\Delta E$ transferred from a particle with the mass $m$ and incidence energy $E_{\mathrm{i}}$ to a cube with effective mass $M$ (Eq. 2.4).

$$
\begin{gathered}
m \nu_{\mathrm{i}} \sin \vartheta_{\mathrm{i}}=m \nu_{\mathrm{f}} \sin \vartheta_{\mathrm{f}} \\
m \nu_{\mathrm{i}} \cos \vartheta_{\mathrm{i}}=m \nu_{\mathrm{f}} \cos \vartheta_{\mathrm{f}}+M \nu_{\mathrm{s}} \\
\frac{1}{2} m \nu_{\mathrm{i}}^{2}=\frac{1}{2} m \nu_{\mathrm{f}}^{2}+\frac{1}{2} M \nu_{\mathrm{s}}^{2} \\
\frac{\Delta E}{E_{\mathrm{i}}}=\frac{\nu_{\mathrm{i}}^{2}-\nu_{\mathrm{f}}^{2}}{\nu_{\mathrm{i}}^{2}}=\frac{4 \cdot m M}{(m+M)^{2}} \cdot \cos ^{2} \vartheta_{\mathrm{i}}
\end{gathered}
$$

Atoms colliding with the surface at decreasing incidence angle experience an enhanced energy loss $E_{\text {loss }}=\Delta E=E_{\mathrm{i}}-E_{\mathrm{f}}$. The maximum energy loss is expected for collisions perpendicular to the surface $\left(\vartheta_{\mathrm{i}}=0^{\circ}\right)$, which is determined by the Baule limit:

$$
\frac{\Delta E}{E_{\mathrm{i}}}=1-\frac{(m-M)^{2}}{(m+M)^{2}}
$$

Grimmelmann et al. extended the model by addition of an attractive interaction using a square well gas-surface potential of depth $W$ and initial thermal motion of the surface cubes allowing trapping and energy tranfer from the surface to the colliding atom.6/34

$$
\Delta E=\frac{4 \cdot m M}{(m+M)^{2}} \cdot\left[E_{\mathrm{i}} \cdot \cos ^{2} \vartheta_{\mathrm{i}}+W\right]-\frac{m(2 M-m)}{(m+M)^{2}} \cdot 2 k_{\mathrm{B}} T_{\mathrm{s}}
$$

When the energy transfer $\Delta E$ is higher than normal incidence energy of the impining atom, trapping into the potential well can occur. 6 . With increasing surface temperatures the energy loss of the scattered atom and trapping probability is reduced.

The solely parallel momentum conversation implies that the scattering angle is affected by the degree of normal momentum transfer. Thus, energy transfer from the atom to the surface will result in scattering away from the specular angle and towards the surface. In contrast, net energy transfer from the surface to the atom results in scattering angles towards the surface normal.

The hard cube model frequently gives rise to reasonable first rough estimations for the energy transfer, especially for non-reactive atom-surface interactions with low atomic incidence energies and angles. $6[34$

An example is given for in-plane scattering studies of Ar atoms from $\operatorname{Ag}(111)^{35 \mid 36}$ at various incidence energies (left panel) and surface tempertures (right panel) (Fig. 2.2). 


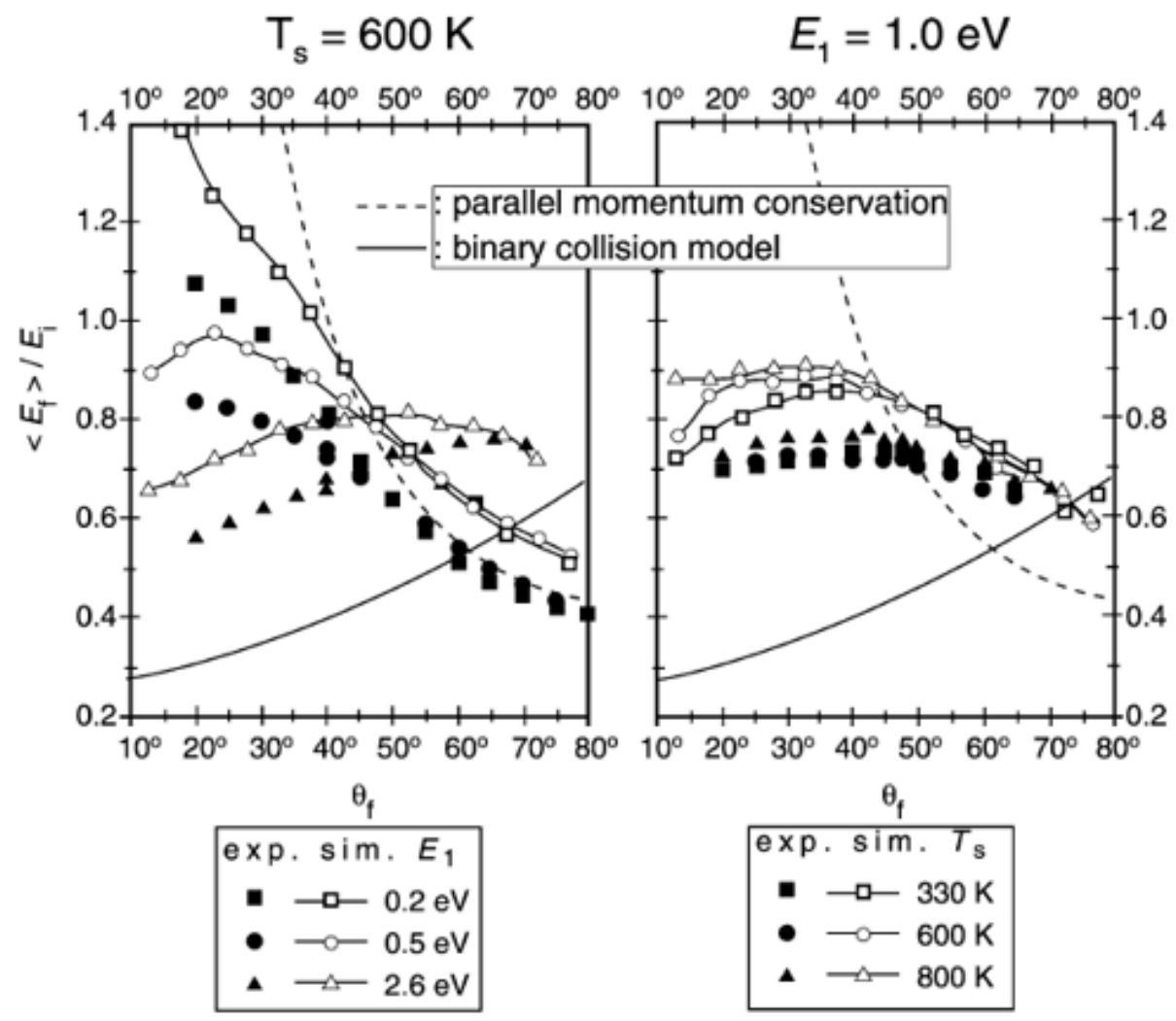

Figure 2.2: Relative final average energy distribution with respect to the scattering angle $\theta_{\mathrm{f}}$ derived from in-plane scattering experiments of $\mathrm{Ar}$ from $\mathrm{Ag}(111)$ at an incidence angle of $40^{\circ}$ (filled symbols) and molecular dynamic simulations (open symbols). Left panel: Results obtained at $T_{\mathrm{s}}=600 \mathrm{~K}$ for various incidence energies $E_{\mathrm{i}}=0.2-2.6 \mathrm{eV}$. Right panel: Results obtained at $E_{\mathrm{i}}=1.0 \mathrm{eV}$ for various surface temperatures $T_{\mathrm{s}}=330-800 \mathrm{~K}$. Dashed and solid lines indicate the energy transfer calculated by the hard cube model (parallel momentum conversation) and a binary hard sphere collision model, respectively. From Kleyn. 31 
The determined relative final average energy of the scattered Ar atoms with respect to the scattering angle obtained from the experiment (filled symbols) and molecular dynamic simulations (open symbols) are compared to a simple hard cube model (dashed line: parallel momentum conversation according to Eq. 2.4 ) ).

The hard cube model predicts the final average energy of the scattered Ar atom reasonably well for low incidence energies (black filled squares). However, for high incidence energy (filled triangles) the model is no longer applicable as the scattering dynamics change and the final average scattering energy starts to increase with the scattering angle. In this case, the atoms experience a larger potential corrugation as the turning point of the incidence atoms shifts with increasing energy closer to the surface ion cores. 37

More sophisticated models which incorporate surface corrugation as the washboard model developed by Tully 38 or a binary collision model, treating the atom surface interaction as a hard sphere collision (see Fig. 2.1 b)), yield better agreements with the experimental data for high incidence energies (black line).

The hard sphere collision model assumes that the incoming atom collides with an individual surface atom with the mass $M$. Parallel momentum conversation is no longer valid, thus, the final translational energy of the scattered atom becomes a function of the total scattering angle $\vartheta=\vartheta_{\mathrm{i}}+\vartheta_{\mathrm{f}} \cdot 36[37$

$$
\frac{E_{\mathrm{f}}}{E_{\mathrm{i}}}=\frac{1}{\left(1+\frac{m}{M}\right)^{2}} \cdot\left[\sqrt{1-\left(\frac{m}{M}\right)^{2} \cdot \sin ^{2}(\vartheta)}-\frac{m}{M} \cos (\vartheta)\right]^{2}
$$

According to this model, the translational energy loss decreases (final translation energy increases) with increasing scattering angle, which gives much better agreements to experiments performed at higher incidence energies (Fig. 2.2, solid line).

In a first approximation, the surface atoms are assumed to be isolated from one another and thus the surface mass $M$, which the colliding atom experiences is equal to the single surface atom (soft surface limit). Due to the binding of the surface atoms the surface is in most of the cases more rigid, indicating that the incoming atom experience collisions with a surface atom of an higher effective mass. Experiments of Ar atoms scattered from $\mathrm{Pt}(111)$ showed that better agreements with binary collision models are obtained when the surface mass increases to $M=2.5 \cdot M_{\mathrm{Pt}}$ (with $M_{\mathrm{Pt}}$ mass of a single platinum atom). 35 


\subsubsection{Born-Oppenheimer approximation (BOA)}

A full description of the atom-surface interaction involves the construction of a potential energy surface (PES). The time-independent non-relativistic Schrödinger equation in full space of electron and nuclear coordinates determines the potential energy $E$ between a collection of atoms with respect to their geometry. $33 \mid 39$

$$
\hat{H} \Psi(\mathbf{r}, \mathbf{R})=E \Psi(\mathbf{r}, \mathbf{R})
$$

$\Psi(\mathbf{r}, \mathbf{R})$ is the total wave function of nuclei and electron in dependence of their position $\mathbf{R}$ and $\mathbf{r}$, respectively. $\hat{H}$ is the Hamilton-operator:

$$
\begin{aligned}
\hat{H} & =-\sum_{I=1}^{N} \frac{\hbar^{2} \nabla_{I}^{2}}{2 M_{I}}-\sum_{i=1}^{n} \frac{\hbar^{2} \nabla_{i}^{2}}{2 m_{\mathrm{e}}}+\sum_{I<J} \frac{Z_{I} Z_{J} e^{2}}{\left|\mathbf{R}_{I}-\mathbf{R}_{J}\right|}+\sum_{i<j} \frac{e^{2}}{\left|\mathbf{r}_{i}-\mathbf{r}_{j}\right|}-\sum_{i, I} \frac{Z_{I} e^{2}}{\left|\mathbf{r}_{i}-\mathbf{R}_{I}\right|} \\
& =-\sum_{I=1}^{N} \frac{\hbar^{2} \nabla_{I}^{2}}{2 M_{I}}+\hat{H}_{e l}(\mathbf{r} ; \mathbf{R})=-\hat{T}_{\mathrm{N}}+\hat{H}_{e l}(\mathbf{r} ; \mathbf{R})
\end{aligned}
$$

where $M_{I}$ denotes the mass of the nucleus $I$ with the nuclear charge $Z_{I}$. The electronic mass and elementary charge are denoted by $m_{e}$ and $e$, respectively.

Solving the full Schrödinger equation involves extraordinary high computational costs, and thus, it is in most of the cases not performed. The Born-Oppenheimer approximation 32 (adiabatic approximation) simplifies the Hamiltonian based on the assumption that the motion of atomic nuclei and electrons can be separated:

Due to the large mass difference (mass of one proton is 1836 times the mass of an electron) electrons respond instantaneously to the much slower motions of the nuclei, thus, when the nuclei moves through a position $R$, the electrons readjust to the same optimum configuration that they would have for a stationary nuclei at position $R .33$

Separation of the Hamiltonian into a kinetic energy operator for the nuclei $\hat{T}_{\mathrm{N}}$ and an electronic Hamiltonian $\hat{H}_{e l}(\mathbf{r} ; \mathbf{R})$ at a fixed nuclei position $R$, enables to construct adiabatic electronic wave functions $\phi_{n}(\mathbf{r} ; \mathbf{R})$ to be eigenfunctions of the electronic Hamiltonian.

$$
\hat{H}_{e l}(\mathbf{r} ; \mathbf{R}) \phi_{n}(\mathbf{r} ; \mathbf{R})=\mathcal{E}_{n}(\mathbf{R}) \phi_{n}(\mathbf{r} ; \mathbf{R})
$$

Note $\phi_{n}(\mathbf{r} ; \mathbf{R})$ depends only parametrically on the position of the nuclei $\mathbf{R}$. The resulting eigenvalue $\mathcal{E}(\mathbf{R})$ of the electronic state $n$ is the adiabatic (or Born-Oppenheimer) potential 
energy surface.

The exact wave function $\Psi(\mathbf{r}, \mathbf{R})$ can be expressed now as a linear combination of the basis functions $\phi_{n}(\mathbf{r} ; \mathbf{R})$ and the nuclear wave function $\chi_{n}(\mathbf{R})$.

$$
\Psi(\mathbf{r}, \mathbf{R})=\sum_{i} \phi_{n}(\mathbf{r} ; \mathbf{R}) \chi_{n}(\mathbf{R})
$$

Substitution of the total wave function (Eq. 2.12) into the Schrödinger equation (Eq. 2.10), multiplication from the left by $\phi_{n}^{*}(\mathbf{r} ; \mathbf{R})$ and integration over all electronic coordinates $\mathbf{r}$ results in the following expression:

$$
\left[-\sum_{I=1}^{N} \frac{\hbar^{2} \nabla_{I}^{2}}{2 M_{I}}+\mathcal{E}_{n}(\mathbf{R})+\frac{\hbar^{2}}{2} \sum_{m}\left[2 \hat{T}_{m n}^{(1)}(\mathbf{R})+\hat{T}_{m n}^{(2)}(\mathbf{R})\right]\right] \chi_{n}(\mathbf{R})=E \chi_{n}(\mathbf{R})
$$

where $\hat{T}_{m n}^{(1)}$ and $\hat{T}_{m n}^{(2)}$ are the first (momentum) and second (kinetic energy) non-adiabatic coupling terms:

$$
\begin{aligned}
& \hat{T}_{m n}^{(1)}=\sum_{I} \frac{1}{M_{I}}\left\langle\phi_{m}\left|\nabla_{\mathbf{R}_{I}}\right| \phi_{n}\right\rangle \cdot \nabla_{\mathbf{R}_{I}} \\
& \hat{T}_{m n}^{(2)}=\sum_{I} \frac{1}{M_{I}}\left\langle\phi_{m}\left|\nabla_{\mathbf{R}_{I}}^{2}\right| \phi_{n}\right\rangle
\end{aligned}
$$

The terms diminish for (1) small nuclear velocities, (2) gradual changes of the adiabatic wave functions with the nuclear coordinates and (3) when the adiabatic wave functions are well separated energetically.6 Neglection of the first and second derivative results in the Born-Oppenheimer approximation:

$$
\left[-\sum_{I=1}^{N} \frac{\hbar^{2} \nabla_{I}^{2}}{2 M_{I}}+\mathcal{E}_{n}(\mathbf{R})\right] \chi_{n}(\mathbf{R})=E \chi_{n}(\mathbf{R})
$$

Deriving the potential energy function $\mathcal{E}_{n}(\mathbf{R})$ from Eq. 2.11 for each required nuclear coordinate $\mathbf{R}$ separates the nuclear and electronic motion. Thus, dynamics are described simply as nuclear motion on a single adiabatic PES. 


\subsubsection{Molecular dynamics (MD) and ab initio molecular dynamics (AIMD)}

For a complete understanding of gas-surface interactions, dynamical simulations have to be performed. Full quantum mechanical (QM) treatments would be ideal to comprehensively describe atomic motion on surfaces. However, apart from a few exceptions, 40 this approach is currently too cumbersome for a general application, thus, classical methods are often employed giving rise to satisfactory results with respect to explaining experimental data. 39

Molecular dynamic (MD) simulations treat the atomic motion classically either by Newton's equation of motion

$$
M_{i} \frac{\partial^{2}}{\partial t^{2}} \mathbf{R}_{i}=-\frac{\partial}{\partial \mathbf{R}_{i}} \mathcal{E}_{n}(\mathbf{R}),
$$

or Hamilton's equation of motion

$$
\dot{q}=\frac{\partial H}{\partial p} \quad \dot{p}=-\frac{\partial H}{\partial q}
$$

with $q$ and $p$ as generalized coordinates and momenta, respectively. By numerically integration over the equation for specific trajectories and integration over initial conditions of many trajectories, quantitatively accurate results can be obtained at relative short computing time (e.g. Figure 2.2, open symbols).

A general problem of this approach is that only one potential energy surface is considered in the force field leading to inaccuracies. For a more accurate description, one needs a dynamic description of the energy potential. This problem can be circumvented in $a b$ initio molecular dynamic (AIMD) simulations, in which the gradients of the potential needs to be calculated only for one particular configuration at any time. The forces necessary to integrate the classical equations of motions are determined on-the-fly by electronic structure calculations in each step of the simulation. The electronic structure can be calculated by quantum dynamics, e.g. using density functional theory (DFT). However, simulations in the AIMD approach are still computationally demanding, thus, most of AIMD studies are limited to simulations of a few hundred trajectories, which is often insufficient in order to obtain good statistics. However current studies show promissing results (e.g. $\mathrm{H}_{2}$ dissociation on noble metals). $\left.39 \mid 41\right]$ 


\subsubsection{Failure of the Born-Oppenheimer approximation}

The previously employed Born-Oppenheimer approximation is no longer valid when the coupling terms in Eq. 2.13 become relevant. This is in particular the case for interaction processes when metal surfaces are involved. Metal surfaces provide a continuum of electronic states (no band gap), thus, electron-hole pair (ehp) excitation above the Fermi level is possible, therefore, condition (3) for the BOA is formally violated.

The Born-Oppenheimer breakdown due to generation of electronic excitations in metals was observed in many experimental studies, 42 such as in Schottky-diodes or in metalinsulator-metal (MIM) devices, where atomic or molecular adsorption induces electrical chemicurrents. 4449

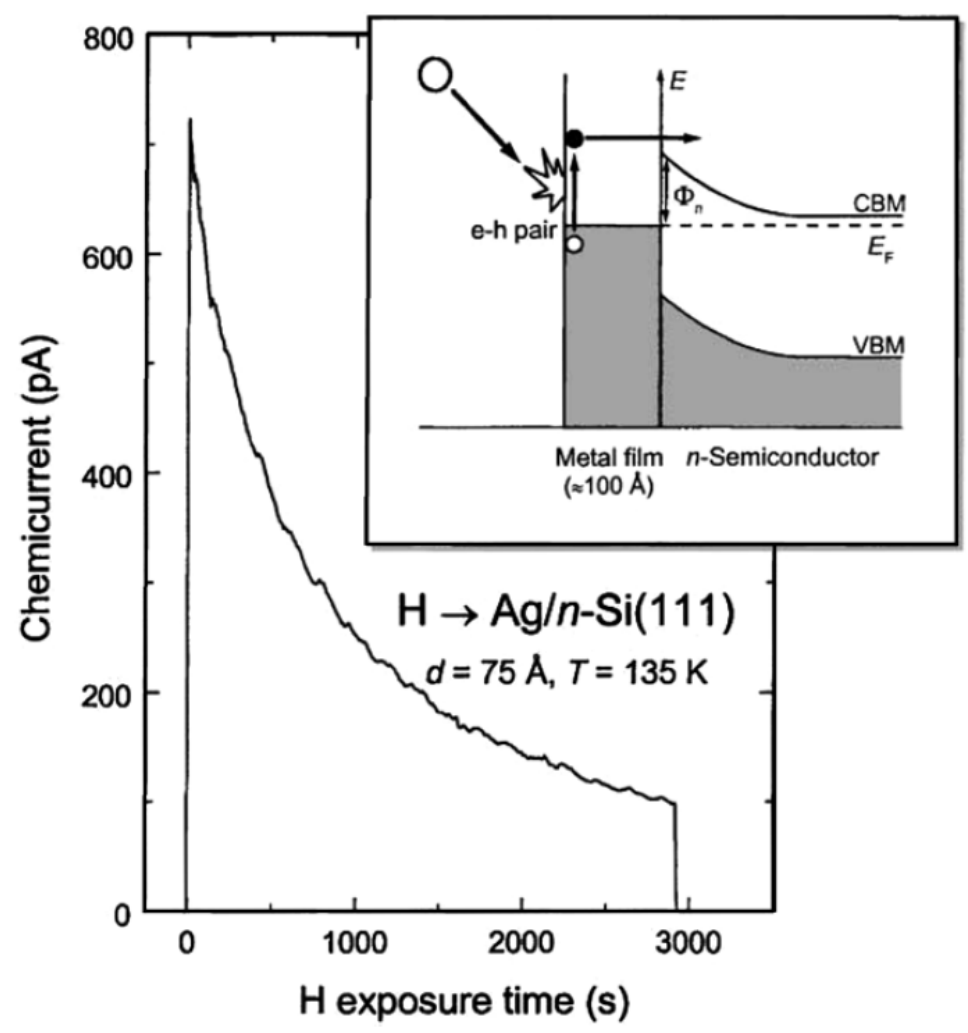

Figure 2.3: Chemicurrent measurement from $\mathrm{H}$ atoms absorption on a $\mathrm{Ag} / \mathrm{n}-\mathrm{Si}(111)$ diode with a Ag film thickness of $75 \AA$ (substrate temperture $135 \mathrm{~K}$ ). Inset: Function principle of hot electrons detection created by ehp excitation with Schottky diodes. From Nienhaus. 44

A Schottky diode device (metal-semiconductor contact) can be formed by a thin transient metal film (film thickness $d \approx 100 \AA$ ) deposited on n-type or p-type $\mathrm{Si}(111)$. The Metal/Si interface forms a Schottky barrier $\Phi_{\mathrm{n}}$ due to space charge effects. 
The inset in Fig. 2.3 illustrates the functional principle of a n-type Schottky diode. The Fermi level $E_{\mathrm{F}}$, the conduction band minimum $(\mathrm{CBM})$, the valence band maximum $(\mathrm{VBM})$ and Schottky barrier $\Phi_{\mathrm{n}}$ are label in the figure. The adsorption process of an incoming atom or molecule leads to excitation of electron-hole pairs. The excited electron travels ballistically through the thin metal film and reaches the semiconductor. When the energy of the excited electron exceeds the Schottky barrier (here $\mathrm{Ag} / \mathrm{Si}(111)$ : $\left.\Phi_{\mathrm{n}}=0.5-0.55 \mathrm{eV}\right), 45 \mid 46$ the hot electrons can be detected as a current, called chemicurrent. Using p-type Schottky diodes, the generated holes can be detected.

Fig. 2.3 shows a measurement of induced chemicurrents from hydrogen atom exposure upon a n-type $\mathrm{Ag} / \mathrm{Si}(111)$ diode. After the beam shutter is opened ( $t=0 \mathrm{~s})$ subsequently a chemicurrent of $720 \mathrm{pA}$ is detected which decays exponentially to a steady-state current of approximately $100 \mathrm{pA}$. At $t=2900 \mathrm{~s}$ the beam shutter is closed again and the current drops instantly to zero. The exponential decay results from a occupation of free surface sites by atomic hydrogen which follows first-order kinetics. The final steady-state currents are explained by a balance between adsorption and abstraction of $\mathrm{H}$ atoms.

Nienhaus et al. further studied the isotope effect exposing $\mathrm{H}$ and $\mathrm{D}$ atoms on a n-type $\mathrm{Ag} / \mathrm{Si}(111)$ Schottky diode. 46 They observed a three times higher chemicurrent for $\mathrm{H}$ atom adsorption although the $\mathrm{D}$ atom flux was approximately twice as large. This isotope effect is explained by a lower efficiency in deexcitation of highly excited vibrational states formed under chemisorption for D-Ag bond due to a smaller energy spacing between the vibrational states.

Additional studies investigating chemicurrents induced by various atoms and molecules revealed that the probability of exciting detectable ehps correlates linearly with the adsorption energy. 47

Treating reaction dynamics with non-adiabatic transition is far from trivial. One promissing approach is to descripe non-adiabatic effects in terms of electronic friction which are superimposed on adiabatic dynamics. 49 Trail et al. developed an electronic friction theory simulating chemicurrents induced by $\mathrm{H}$ and D atom exposure on Ag. 50 The theoretical predictions were in reasonable good agreement with the experimentally observed values. Janke et al. developed a theoretical model treating mechanical energy transfer to metal lattice motion as well as electronic excitation of a metal surface.51 This model contains self consistent molecular dynamic (MD) simulations carried out on a constructed global full-dimensional potential energy surface (PES) based on effective medium theory (EMT) and fitted to ab initio electronic energy values computed by density functional theory (DFT) at the generalized gradient approximation (GGA) level. EMT intrinsically 
includes electron densities, thus, electronically non-adiabatic effects can be described self-consistently on the level of the local-density electronic friction approximation (LDFA). This model is able to explain the isotope effect which was observed in the chemicurrent measurement with relatively high accuracy. 52 In this thesis, theoretical simulations derived from the model are compared to the experimentally obtained data of H/D atoms scattered from transition metal surfaces. 


\subsection{Relevant defined surfaces}

In this thesis, experiments were performed on single crystalline gold, platinum, silver, palladium, nickel and copper metal surfaces at a (111) surface cut as well as on a single crystalline insulting $\alpha-\mathrm{Al}_{2} \mathrm{O}_{3}(0001)$ surface. The following section will provide basic information of the atomic and electronic structure aforementioned surfaces.

\subsubsection{Metal surfaces}

Transition metals of the copper and nickel group all crystallize face-centered cubic $(f c c)$. This crystal structure corresponds to a $A B C A B C$-stacking, meaning that every third layer is identical. A cut along the (111) face leads to one of the most possible densely packed atomic planes shown in Figure 2.453

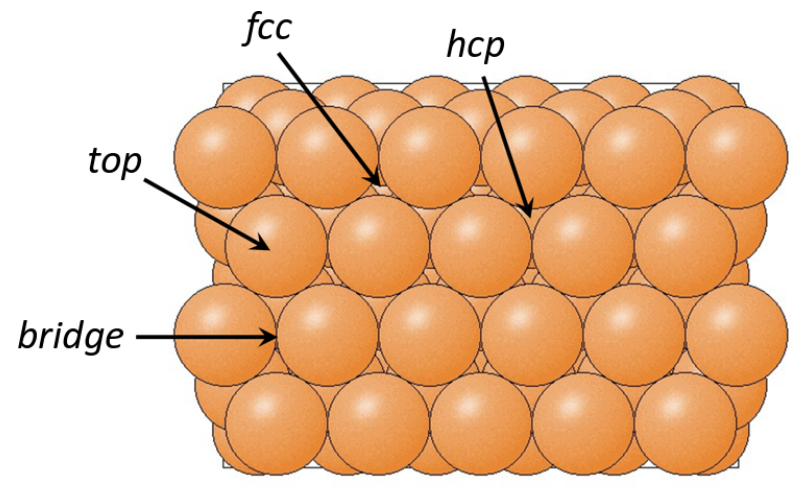

Figure 2.4: Top view of the atomic surface structure of a single crystal $f c c(111)$ metal surface. The surface adsorption sites top, bridge and the two hollow sites $f c c$ and $h c p$ are labeled in the figure. The image was generated using the software SURFACE EXPLORER based on BALSAC.54

The surface structure has a three-fold symmetry leading to four geometrically adsorption sites on the surface labeled in the figure. The top-site corresponds to a bond directly to only one metal atom while the bridge-site is located between two neighboring surface atoms. Two hollow sites result from the surface symmetry structure: 1. hexagonal closed-packed $(h c p)$-hollow site, where an atom is embedded in the second atomic plane and 2. face-centered cubic $(f c c)$-hollow site, where an atom sits two layers below in the third atomic plane.

While these adsorption sites exist for ideal $f c c(111)$ surfaces, it should be noted that surface atoms tend to minimize the surface energy by rearranging their surface position leading to a reconstructed surface which usually includes a less ordered structure. However, 
a reconstruction of clean $f c c(111)$ metal surfaces does not occur at room temperature. With respect to the investigated surfaces in this studies, so far only for $\mathrm{Au}(111)$ a surface reconstruction was observed due to the high ductility and malleability of gold. 55 The gold surface atoms of the top layer compress to a $22 \times \sqrt{3}$ unit cell with a herringbone structure on a few hundred nanometer length scale. 56

Further important characteristics of metals of the copper and nickel group, such as the atomic mass, lattice constant, work function and electronic configuration, are listed in the following table.

Table 2.1: Mass, work function and electronic configuration of the investigated $d$-metals listed according to atomic mass. $\frac{57}{57}$

Metal Atomic Mass Lattice constant Work Function Electronic Configuration

\begin{tabular}{lcccc} 
& $/ \mathrm{au}$ & $/ \AA$ & $\Phi / \mathrm{eV}$ & \\
\hline \hline $\mathrm{Au}(111)$ & 197.0 & 4.08 & 5.31 & {$[\mathrm{Xe}] 4 \mathrm{f}^{14} 5 \mathrm{~d}^{10} 6 \mathrm{~s}^{1}$} \\
$\mathrm{Pt}(111)$ & 195.1 & 3.92 & 5.93 & {$[\mathrm{Xe}] 4 \mathrm{f}^{14} 5 \mathrm{~d}^{9} 6 \mathrm{~s}^{1}$} \\
$\operatorname{Ag}(111)$ & 107.9 & 4.09 & 4.74 & {$[\mathrm{Kr}] 4 \mathrm{~d}^{10} 5 \mathrm{~s}^{1}$} \\
$\mathrm{Pd}(111)$ & 106.4 & 3.89 & 5.60 & {$[\mathrm{Kr}] 4 \mathrm{~d}^{10}$} \\
$\mathrm{Cu}(111)$ & 63.5 & 3.61 & 4.94 & {$[\mathrm{Ar}] 3 \mathrm{~d}^{10} 4 \mathrm{~s}^{1}$} \\
$\mathrm{Ni}(111)$ & 58.7 & 3.52 & 5.35 & {$[\mathrm{Ar}] 3 \mathrm{~d}^{8} 4 \mathrm{~s}^{2}$}
\end{tabular}

One important characteristic of the investigated metals is the work function, which corresponds to the energy needed to transfer an electron from the bulk to vacuum. Within a group the work function increases with the atomic mass and is generally higher for the $\mathrm{Ni}$ group than for the $\mathrm{Cu}$ group for metals of the same period.

The electronic ground configuration is similar for all metals of the $\mathrm{Cu}$ group but differs in the $\mathrm{Ni}$ group. Consistently metals of the $\mathrm{Cu}$ group possess a fully occupied $d$-shell and a half filled $s$-shell, while for the Ni group no regular pattern for the valence shell electron is observed. Platinum has nine electrons in the valence $d$-shell and a half-filled $6 s$-shell. Palladium exhibits a fully occupied valence $4 d$-shell and nickel has eight electrons in the $d$-shell and a fully occupied $4 s$-shell.

These different valence electronic states further impact the bulk densitiy of states (DOS) around the fermi level. Copper group metals have similar low densities of $s p$-derived bulk electronic states at the Fermi level. High DOSs with $d$ character lay all 1.5-3 eV below the Fermi level. In contrast, nickel group metals exhibit a high DOS with $d$ electronic character at the Fermi level. 58

The open character of a surface yields a different electronic structure in the top surface layers. Therefore, it is crucial to investigate the electronic states at the surface. According 
to high-angle resolved photoemission studies, all six $f c c(111)$ transition metals exhibit an additional surface state close to the Fermi level. 5960

\subsubsection{Aluminum oxide surface}

Aluminum oxide or alumina is a ceramic material and an insulator with a band gap of $8.8 \mathrm{eV}$ at room temperature.61 Due to its material properties, such as high melting point, extreme hardness, high electrical resistivity, thermal conductivity and chemical stability, alumina is used in many technical applications, e.g. for passivation and coating or as a substrate for thin film preparation, as catalyst support, for electronic packaging and in microelectronics. $62[63$ The thermodynamically stable phase of aluminum oxide is the $\alpha$ modification, forming the closest packing of all known phases with a density of $3.97 \mathrm{~g} / \mathrm{cm}^{3}$.

Bulk $\alpha-\mathrm{Al}_{2} \mathrm{O}_{3}$ has a rhombohedral symmetry composed of two $\mathrm{Al}_{2} \mathrm{O}_{3}$ molecular units. It can also be described as a hexagonal unit cell containing six formula units. Here, the $\mathrm{O}^{2-}$ anions are equidistant from each other forming almost a perfect hexagonal closed-packing with $A B A B$-stacking perpendicular to the (0001) plane. The $\mathrm{Al}^{3+}$ ions occupy $2 / 3$ of the octahedral holes at interstitial positions between two oxygen layers with an $f c c$-type abcabc-stacking. This leads to a hexagonal unit cell of $12 \mathrm{Al}$ and six $\mathrm{O}$ layers with $a A b c B a b A c a B b c A a b B c$-stacking. 64

In principle, the surface can either terminate with an oxygen layer $(A a b)$ or an aluminum layer $(a A b)$, which was broadly discussed in the past. Theoretical calculations predict that the surface energy for non-polar Al-O-Al terminated surfaces is half as large as the O-Al-Al termiated surface with a non-zero dipol moment.65 Latest experimental results revealed that for a $\alpha-\mathrm{Al}_{2} \mathrm{O}_{3}$ surface, stored in UHV environment, the surface is Al-O-Al terminated with a large interlayer contraction between the first two layers (nearly factor of two). $63 \mid 64[66$

Fig. 2.5 illustrates the most probable atomic structure of $\alpha-\mathrm{Al}_{2} \mathrm{O}_{3}(0001)$ at surface top view with $\mathrm{Al}-\mathrm{O}-\mathrm{Al}$ termination. The $\mathrm{Al}$ and $\mathrm{O}$ atoms are labeled according to their layer position.

Although the surface terminates with $\mathrm{Al}$ atoms, it is an open surface where a huge proportion of the oxygen atoms from the $2^{\text {nd }}$ and $5^{\text {th }}$ layer are visible on the surface due to the small ion radius of $\mathrm{Al}$. In addition, the Al-O distance of the first layer is strongly contracted.

It should also be noted that experiments showed that hydrated $\alpha-\mathrm{Al}_{2} \mathrm{O}_{3}(0001)$ terminate 


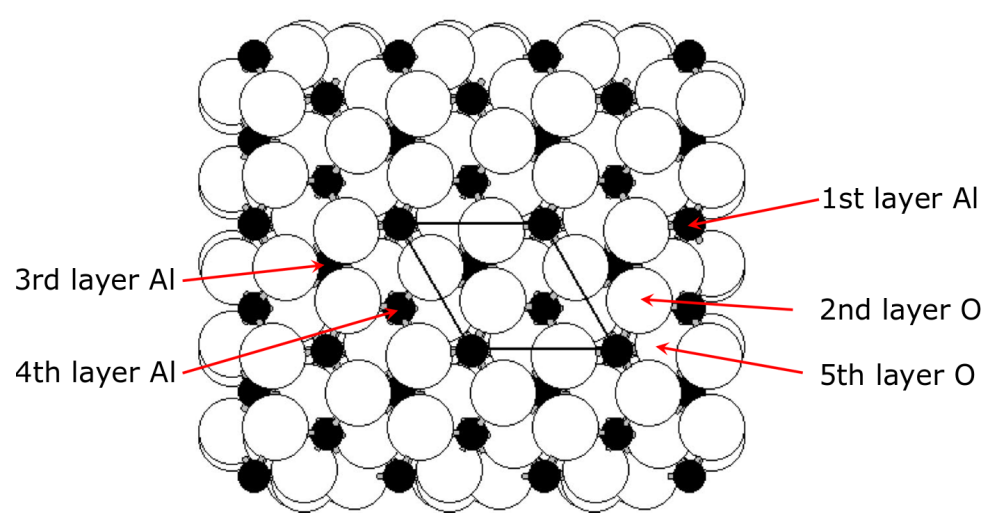

Figure 2.5: Plane view of $\alpha-\mathrm{Al}_{2} \mathrm{O}_{3}(0001)$ atomic surface structure. Al atoms: small filled black circles, $\mathrm{O}$ atoms: large white circles. The red arrows label the atom layer postions. The unit cell is marked by the black lines froming a rhombus. Illustration derived from Soares et al. 64

with an oxygen layer, however, this is not the most stable configuration for samples cleaned in UHV.67

\subsubsection{Graphene surface}

Graphene is a two-dimensional modification of carbon, in which each carbon atom is surrounded by three atoms at a C-C-C bond angle of $120^{\circ}$, yielding a honeycomb lattice structure (Fig. 2.6 a).

a)

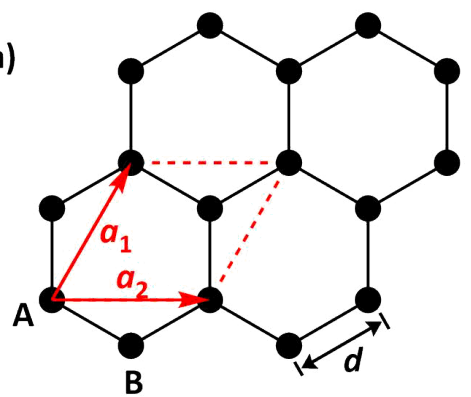

b)

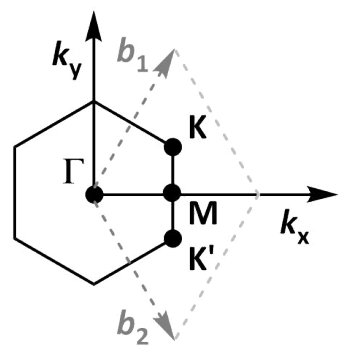

Figure 2.6: a) Honeycomb lattice structure of graphene. The unit cell (red rhombus) formally consists of two atoms A and B with the lattice unit vectors $a_{1}$ and $a_{2}$. b) Reciprocal lattice, which illustrates the first Brillouin zone with the reciprocal-lattice vectors $b_{1}$ and $b_{2}$. The $\Gamma$ point denotes the center of the Brillouin zone, $K$ and $K^{\prime}$ denote the corner points (named Dirac points), and $M$ marks the center point of two Dirac points. 68 
The hexagonal lattice can be considered as two interleaving triangular lattices of two carbon atoms $\mathrm{A}$ and $\mathrm{B}$, which are shifted by the $\mathrm{C}-\mathrm{C}$ bond length $d=1.42 \AA$. The unit cell (red rhombus) consists of two atoms with the lattice vectors $a_{1}$ and $a_{2}$. The absolute number of both lattice vectors is $a=2.46 \AA .68$

The reciprocal lattice is illustrated in Fig. $2.6 \mathrm{~b}$. The center $\Gamma$ of the first Brillouin zone and the corner points $K$ and $K^{\prime}$ are labeled in the figure.

Two neighboring carbon atoms form a $\sigma$-bond which results from $\mathrm{sp}^{2}$ hybridization of the $\mathrm{s}, \mathrm{p}_{\mathrm{x}}$ and $\mathrm{p}_{\mathrm{y}}$ orbitals. These filled $\sigma$-bonds build up a deep valence band of graphene later refered to as $\sigma$-band. The stability of graphene results from its tightly packed carbon atoms and their $\mathrm{sp}^{2}$ hybridization. The unaffected $\mathrm{p}_{\mathrm{z}}$ electron, which is orientated perpendicular to the planare graphene structure, overlaps with neighboring carbon atoms resulting in a $\pi$-bond formation. The $\pi$-bonds overlap forming a conjugated $\pi$-system resulting in a continuous $\pi$ and $\pi^{*}$-band. The resulting bands are half filled and responsible for most of graphene's notable electronic properties, such as the linear dispersion at the Dirac point and its exceptionally high electron mobility. 69

The band structure of graphene calculated by a tight-binding approximation is shown in Fig. 2.7 a. 68 70 The upper and the lower surface represent the conduction and valence
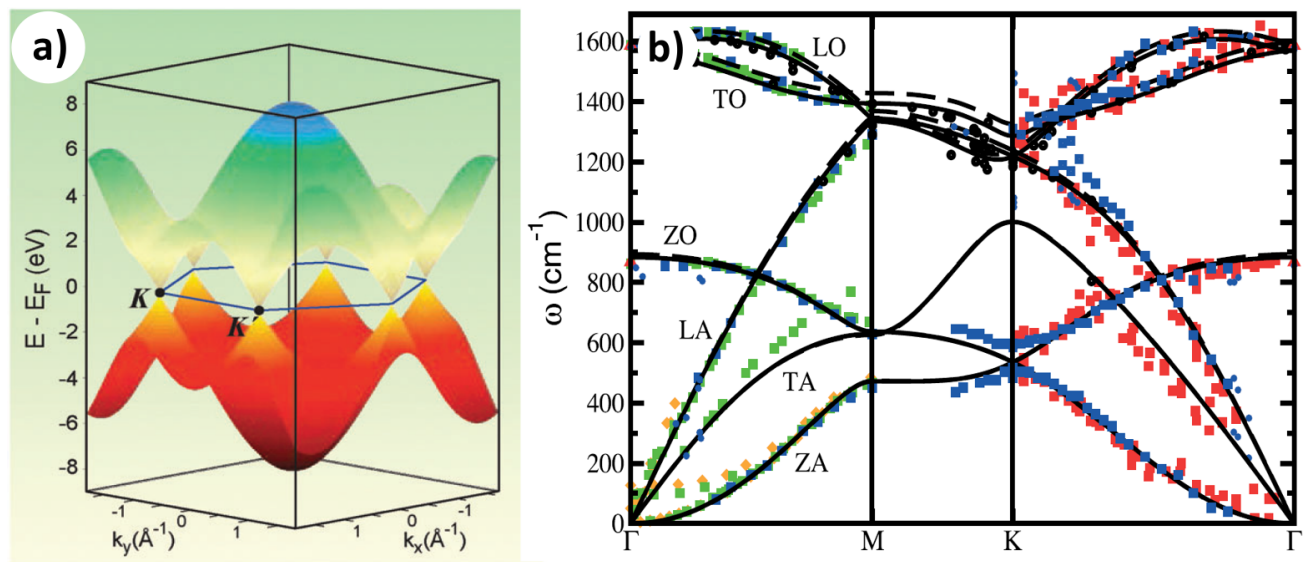

Figure 2.7: a) First Brillouin zone and electronic dispersion of $\pi$ and $\pi^{*}$-states of free-standing graphene. In the vicinity of the $K$ points the branches have linear dispersion. The conduction (upper surface) and valence band (lower surface) intersect close to the $K$ points. Reproduced from Ref. [70] with permission from the PCCP Owner Societies. b) Experimental (symbols) and calculated (solid and dashed lines) phonon dispersion spectra of graphite along the lines $\Gamma$ to $M, M$ to $K$, and $K$ to $\Gamma$ in the Brillouin zone.71

band, respectively. The valence and conduction band meet at the corner points $K$ and $K^{\prime}$ of the Brillouin zone, which therefore are named Dirac points. The Dirac points mark the 
transition between the valence band and the conduction band. This particular structure is the reason why graphene is classified as a zero band gap semiconductor. At the Dirac points (within $1 \mathrm{eV}$ ) the branches have linear dispersion. The dispersion relation near the $K$ points can therefore be expressed by the following equation:

$$
E_{ \pm}(k) \approx \pm \hbar \nu_{\mathrm{F}}|k-K|
$$

where $k$ is the momentum relative to the Dirac points $K$ and $\nu_{\mathrm{F}} \approx 10^{6} \mathrm{~m} / \mathrm{s}$ is the Fermi velocity. The Fermi velocity describes the effective speed of light of the charge carriers in graphene, which leads to the conclusion that the charged carriers behave like relativistic particles. This behavior is responsible for the high electron mobility of graphene at room temperature, which gives rise to a resisitivity of $10^{6} \Omega \cdot \mathrm{cm} .69$

The low mass of the carbon atoms and the strong $\sigma$-bonding in the graphene plane leads to a very high sound velocity, which is responsible for the very high thermal conductivity of graphene. Experiments at near room temperature obtained $\kappa=3080-5150 \mathrm{~W} / \mathrm{mK}$ and a phonon mean free path of $\lambda=775 \mathrm{~nm}$ for a set of graphene flakes, which is one of the highest values ever measured. 69

The phonon dispersion relations of free-standing graphene are believed to be very similar to those of graphite, since the coupling between the graphene planes in graphite are very weak. Fig. 2.7 b) displays the theoretical calculated and experimentally determined phonon dispersion relation of graphite. All modes displayed are labeled as follows: ZA (acoustic) and ZO (optical) correspond to the out-of-plane vibrational modes, whereas TA (transverse acoustic), TO (transverse optical), LA (longitudinal acoustic) and LO (longitudinal optical) can be assigned to in-plane vibrations. The out-of-plane modes intersect at the Dirac points very similar to the electron dispersion relation.

\section{Graphene on metal surfaces}

The highest carrier mobility was measured in free-standing graphene, which can be prepared by mechanical exfoliation from graphite. Unfortunately, with this method only single layer domains in the micrometer range can be achieved, which is not broadly applicably for either industrial processes or scientific studies. In order to produce largerscale graphene domains, chemical vapor deposition (CVD) on different metal substrates has become the most suitable method to produce high-quality graphene. 70 |72 74 Almost perfectly ordered epitaxial overlayer of graphene films have been obtained on a number of hexagonally close-packed surfaces, with domain sizes larger than the terraces of the 
metal substrates. However, the graphene films are often not rotationally aligned to the metal surface, but display often a moiré structure with large periodicity, which results from the lattice mismatch between graphene and the underlying metal. Graphene on a $\mathrm{Ni}(111)$ surface represents an exception as it shows smallest lattice mismatch. The lattice mismatch between graphene and the nickel atom surface bond distance is about $1.2 \%$ (nearest neighbor distance Ni $2.49 \AA$ ), which allows graphene to adapt itself to the $\mathrm{Ni}(111)$ lattice, and thus, forming a (1 x 1) structure on $\mathrm{Ni}(111)$ substrates.
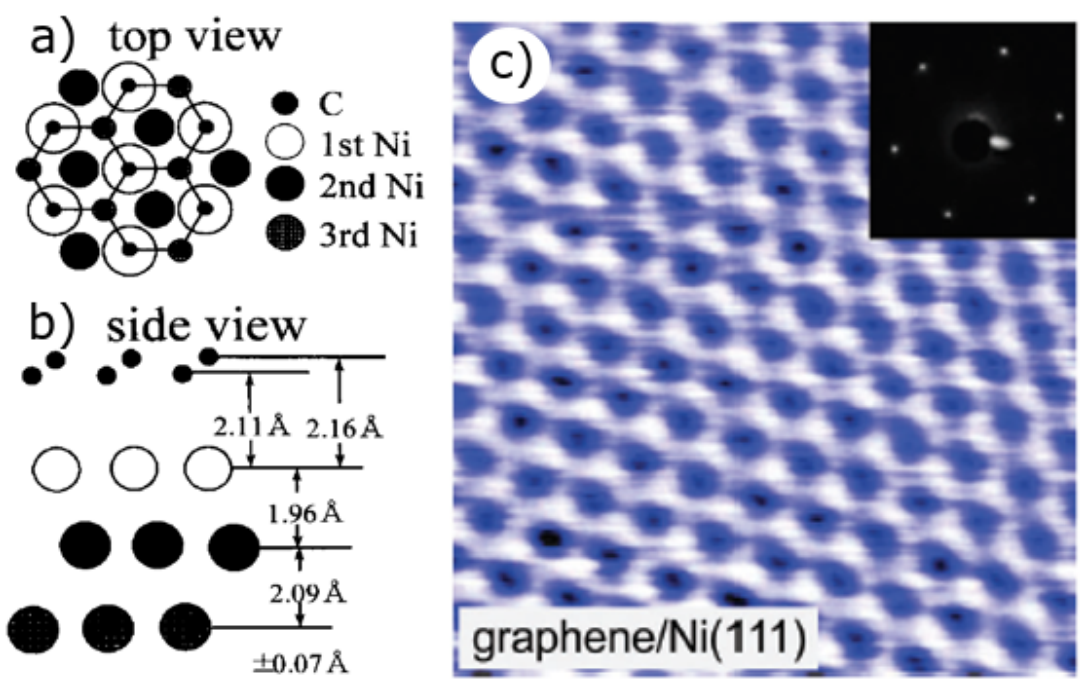

Figure 2.8: Top (a) and side (b) view of (1 x 1) graphene structure on $\mathrm{Ni}(111)$ with a top-fcc configuration. One carbon atom is located on top of the topmost nickel atom and the carbon atom sit on a $f c c$-hollow site of the Ni(111) surface.75 c) STM image of graphene on $\mathrm{Ni}(111)$. Inset: LEED image of $(1 \times 1)$ graphene on $\mathrm{Ni}(111)$. Reproduced from Ref. 70 with permission from the PCCP Owner Societies.

The graphene lattice is predominantly oriented on the nickel surface such that one carbon atom sits on top of the topmost nickel atoms, while the other carbon atom is located on the $f c c$-hollow site of the nickel surface leading to a top-fcc configuration (Fig. 2.8 a) top and b) side view). Figure 2.8 c) shows a STM image of graphene on Ni(111) and the corresponding LEED pattern (inset). A lattice constant of $2.4 \pm 0.1 \AA$ was obtained, which is in good agreement with the in-plane lattice constant of graphite $(2.46 \AA$. Other configurations of the $(1 \times 1)$ oriented graphene, as top-hcp or bridge-top, were also partially observed but seem to be energetically less favorable. $70|72| 75[78$ As a consequence the dominating geometry of graphene on $\mathrm{Ni}(111)$ is the top-fcc configuration.

LEED intensity analysis measured a graphene-metal distance of $2.11 \pm 0.07 \AA$, which was later confirmed by ion scattering analysis and several DFT calculations, which calculated values between 2.0 and $2.1 \AA$. $70|72| 77 / 79 \mid 80$ This distance is much shorter than 
the interlayer spacing in graphite $(3.35 \AA)$, indicating a strong interaction between the nickel substrate and the graphene layer. Nevertheless, there is no evidence yet that the bonding induces a breaking of the conjugated $\pi$-system to form a covalent bond between the graphene and nickel substrate. The height difference of the carbon atoms (Fig. 2.8 b) is below the error limit of the LEED analysis and DFT calculation predict that the graphene layer remains flat upon adsorption on the nickel surface consitent with a surface electron density corrugation of $0.06 \pm 0.02 \AA$ determined by helium atom scattering. 81 In addition, theoretical calculations predict an adsorption energy which is still dominated by van der Waals (vdW) interactions (adsorbtion energy: $67 \mathrm{meV}$, adsorption energy of covalent (classic) chemisorbed species: $0.5-2 \mathrm{eV}) .77|80| 82$
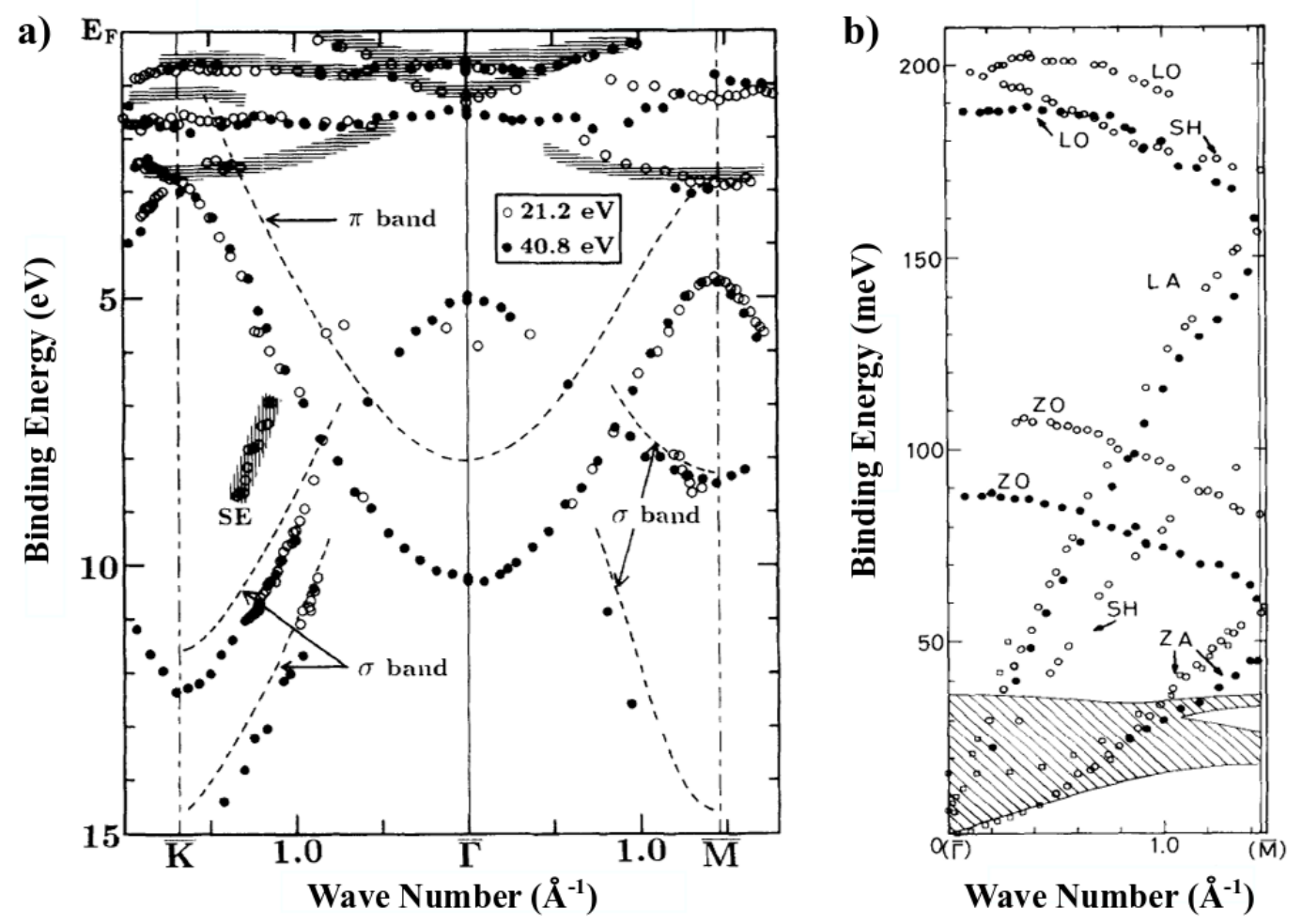

Figure 2.9: a) Electronic band structure of graphene on $\mathrm{Ni}(111)$ obtained by HeI (open circles) and HeII (filled circles) excitation compared to the $\pi$ and $\sigma$ bands of graphite (dashed lines) and the $3 \mathrm{~d}$ band of $\mathrm{Ni}(111)$ (shaded areas). The $\sigma$ and $\pi$ bands of adsorbed graphene are downshifted by approximately $1 \mathrm{eV}$ and $2 \mathrm{eV}$, respectively. 83 b) Phonon dispersion relations of graphene on $\mathrm{Ni}(111)$ (solid circles), graphite (open circles) and $\mathrm{Ni}(111)$ (hatched region) along the $\Gamma$ to $M$ line in the Brillouin zone measured by EELS. The SH line correspond to the transverse acoustic (TA) and transverse optical (TO) lines, which are not visible for graphene on $\mathrm{Ni}(111)$ due to the EELS selection rules.) 84 
Furthermore, experimentally determined $\pi$-band as well as $\sigma$-band structures of graphene on $\mathrm{Ni}(111)$ (open and filled circles, see Fig. 2.9 a) display similar dispersions as known from bulk graphite (dashed lines), which supports the thesis, that the atomic lattice of the adsorbed layer stays intact. However, due to the strong interaction between graphene $\pi$ and $\mathrm{Ni} 3 d$ electrons, the $\pi$-band of the adsorbed graphene is shifted downwards by more than $2 \mathrm{eV}$. The $\sigma$-bands are less affected (downshifted by approximately $1 \mathrm{eV}$ ). The most important change is the band gap opening of the $\pi$-bands around the Dirac points ( $K$ points) of graphene resulting from sublattice symmetry breaking. 76 .

The phonon vibrational spectra of graphene on $\mathrm{Ni}(111)$ are also soften due to the strong substrate-graphene interaction (Fig. 2.9 b) with the out-of-plane optical ZO vibrational mode experiencing the strongest shift $(\sim 20 \mathrm{meV}) .84$ The symmetry breaking of graphene on $\mathrm{Ni}(111)$ (Carbon atoms are located at altering atop and $f c c$-hollow adsorption sides on the $\mathrm{Ni}(111)$ surface) leads to a degeneracy of the $\mathrm{ZO}$ and ZA branch at the $K$ point (not shown in the graphic). 85

Related studies of other metals have shown that the bond strength between the metal surface and the graphene layer depends on the transition metal. For instance, the interaction of graphene on $\operatorname{Ir}(111), \mathrm{Au}(111), \mathrm{Cu}(111), \mathrm{Pt}(111), \mathrm{Al}(111)$ and $\mathrm{Pd}(111)$ is weak, while on $\mathrm{Ni}(111), \mathrm{Co}(0001), \mathrm{Rh}(111)$ and $\mathrm{Ru}(0001)$ it is strong. 76 On weakly bound surfaces, such as $\mathrm{Pt}(111)$, the electronic structure of free-standing graphene is almost intact and only a weakly shifted band crossing of the graphene $\pi$-bands at the Dirac points are observed, while on strongly bound surfaces ( $\mathrm{Ni}, \mathrm{Co}$, etc.), a pronounced energetic splitting of the $\pi$ states is observed. 82 Metal-graphene spacing values between approximately 2.1 and $3.8 \AA$ (strongly to weakly bound surfaces) have been obtained, depending on the metal substrate. The reason for the binding strength variations among the different metal surfaces is yet not fully understood, however there are indications that the systems fall into two classes of physisorbed and quasi chemisorbed graphene. 72

\section{Graphene preparation on $\mathrm{Ni}(111)$}

Epitaxial graphene can be synthesized on $\mathrm{Ni}(111)$ using chemical vapor deposition method via hydrocarbon molecules dosage at elevated temperatures.

Above $600^{\circ} \mathrm{C}$ carbon diffuses into the nickel bulk and multilayer grahene is formed due to carbon segregation from the bulk upon surface cooling. 86 Below $600^{\circ} \mathrm{C}$ the process is self-terminating forming a single graphene layer. 87 Two different growth mechanism for monolayer graphene formation were proposed: $88 \mid 89$ 
1. $T=500$ to $600^{\circ} \mathrm{C}$ : graphene grows on a pure $\mathrm{Ni}(111)$ surface in the absence of any carbide species via replacement mechanisms.

2. Below $500^{\circ} \mathrm{C}$, graphene growth competes with the formation of $\mathrm{Ni}_{2} \mathrm{C}$ carbide on the nickel surface ${ }^{a}$ Carbide formation appears to proceed very rapidly while the graphene growth is rather slow under these conditions. However, the formed $\mathrm{Ni}_{2} \mathrm{C}$ phase can subsequently convert into a graphene layer in an hydrocarbon environment. This process is irreversible due to the higher stability of graphene compared to any nickel carbide species.
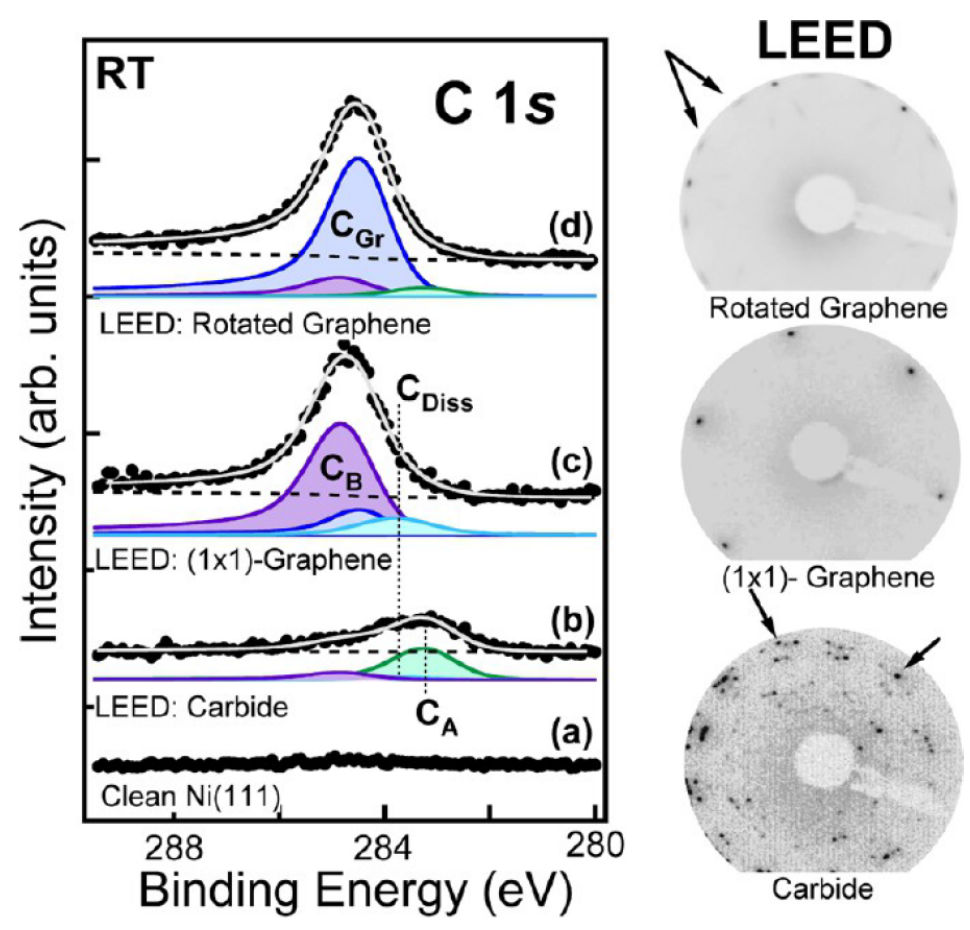

Figure 2.10: Left: C 1s XPS spectra (black circles) of (a) pristine $\mathrm{Ni}(111)$, (b) $\mathrm{Ni}_{2} \mathrm{C}$ phase on $\mathrm{Ni}(111)$, (c) (1 x 1) oriented graphene and (d) rotated graphene phases on $\mathrm{Ni}(111)$. The $\mathrm{C}$ 1s experimental data were separated into XPS data from $\mathrm{Ni}_{2} \mathrm{C}$ $\left(\mathrm{C}_{\mathrm{A}}\right.$, green $),(1 \times 1)$ oriented graphene $\left(\mathrm{C}_{\mathrm{B}}\right.$, purple), rotated graphene $\left(\mathrm{C}_{\mathrm{Gr}}\right.$, blue $)$ and dissociated carbon $\left(\mathrm{C}_{\text {Diss }}\right.$, light-blue) components (dotted line). It becomes obvious, that by the differnet growth mechanism two graphene species are formed $\mathrm{C}_{\mathrm{B}}$ and $\mathrm{C}_{\mathrm{Gr}}$. Right: Corresponding LEED images of the $\mathrm{Ni}_{2} \mathrm{C}$ phase, $\left(\begin{array}{lll}1 & \mathrm{x} & 1\end{array}\right)$ oriented graphene and rotated graphene, respectively. The arrows in the rotated graphene LEED image indicate the extra spots of the resulting moiré pattern. Reprinted with permission from [89]. Copyright 2013 American Chemical Society.

\footnotetext{
${ }^{\mathrm{a}}$ The surface carbide is a single atomic-layer-thick $\mathrm{Ni}_{2} \mathrm{C}$ phase with an ordered $\sqrt{39} R 16.1^{\circ} \times \sqrt{39} R 16.1^{\circ}$ surface structure on $\mathrm{Ni}(111) .88$
} 
Patera et al. $\$ 89$ intensively investigated both growth mechanism by in situ STM (scanning tunneling microscope), XPS (X-ray photoelectron spectroscopy) and LEED. According to these studies, the first reaction mechanism leads to the formation of embedded epitaxial and/or rotated graphene domains on $\mathrm{Ni}(111)$ (for simplicity from now on described as rotated graphene). LEED studies determined a preferential $17^{\circ} \pm 7^{\circ}$ rotation with respect to the $\mathrm{Ni}(111)$ surface (Fig. 2.10, upper LEED image). STM images of the resulting graphene layer show moiré structures with a peak to peak height of $0.2 \AA$ on the Ni(111) surface, which is much smaller than graphene surface corrugations measured on other strongly interacting metal substrates (e.g. Ru or Rh, surface corrugation approximately 1.1 - $1.6 \AA$ ). 85 The second growth mechanism results in almost exclusively ( 1 x 1$)$ oriented monolayer graphene (Fig. 2.10, centered LEED image). C 1s XPS investigations (Fig. 2.10, left) show different binding energies for (1 x 1) oriented graphene (Fig. 2.10, left (c)) and the rotated graphene (Fig. 2.10, left (d)), which indicates that the interaction between the $\mathrm{Ni}(111)$ surface and the different graphene species alters significantly for both modifications.

Experimental and theoretical studies showed that $(1 \mathrm{x} 1)$ oriented graphene is strongly bound on $\mathrm{Ni}(111)$ with a graphene-metal distance of approximately $2.1 \AA$ and a predominant top-fcc adsorption geometry on the $\mathrm{Ni}(111)$ surface. Kozlov et al. 77 performed DFT calculations using a GGA exchange-correlation functional augmented with semiempirical corrections in order to predict the most stable graphene configurations at a graphenesubstrate distance of $2.1 \AA$ and $3.2 \AA$, respectively. They predict that the energetically most favorable configurations of chemisorbed graphene (graphen-substrate spacing: $2.1 \AA$ ) is top-fcc and bridge-top, while physisorbed graphene on $\mathrm{Ni}(111)$ (graphen-substrate spacing: $3.2 \AA$ ) is not sensitive to the lateral positions of $\mathrm{C}$ atoms with respect to the surface Ni atoms. Thus, the aforementioned XPS studies and the theoretical simulations indicate, that the rotated graphene-substrate interaction is reduced to the $(1 \mathrm{x} 1)$ graphene-substrate due to the geometry change. 


\section{Experiment and applied methods}

In this chapter the general experimental set-up and underlying methods of the Rydberg atom tagging (RAT) surface scattering machine, which was used in this thesis for the study of hydrogen atom scattering from well-defined surfaces, will be introduced. A schematic overview of the experiment can be found in Fig. 3.1.

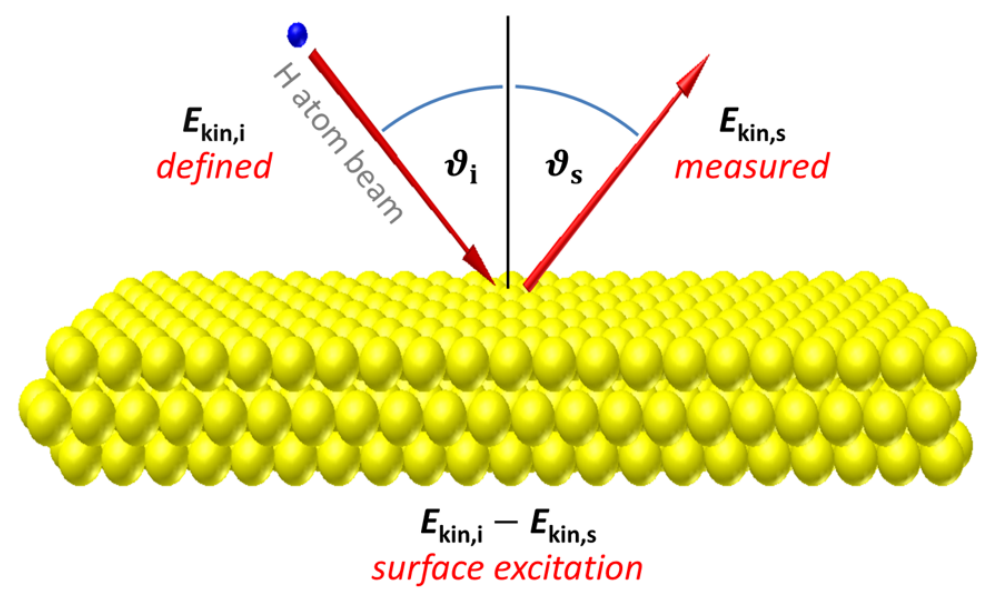

Figure 3.1: General illustration of the $\mathrm{H}$ atom scattering experiment. The incidence energy $E_{\text {kin,i }}$ and incidence angle $\vartheta_{\mathrm{i}}$ of the incoming $\mathrm{H}$ atom beam is experimentally controlled. The final kinetic energy of the scattered $\mathrm{H}$ atoms is measured by TOF with respect to the scattering angle $\vartheta_{\mathrm{s}}$. The surface excitation can be derived from the difference $E_{\text {kin,i }}-E_{\text {kin }, \mathrm{s}}$.

A hydrogen atom beam with extremely narrow kinetic energy range $E_{\text {kin,i }}$ is pointed towards a well-defined surface at an adjusted incidence angle $\vartheta_{\mathrm{i}}$. After the scattering process, the remaining kinetic energies $E_{\mathrm{kin}, \mathrm{s}}$ of the scattered $\mathrm{H}$ atoms are determined with respect to the scattering angle $\vartheta_{\mathrm{s}}$ via $\mathrm{H}$ atom Rydberg tagging time-of-flight (HRTOF). 90 The experiment is carried out under UHV $\left(10^{-10} \mathrm{mbar}\right)$ to increase the mean free pass of the hydrogen atoms and to ensure $\mathrm{H}$ atom scattering from well-defined surfaces. The structure and contaminants of the investigated surfaces are determined with low energy electron diffraction (LEED) and Auger electron spectroscopy (AES). 


\subsection{Rydberg atom tagging surface scattering machine}

The basic experimental apparatus of the Rydberg-Atom-Tagging machine is shown schematically in Fig. 3.2 . In the course of the experiment, the surface is initially cleaned

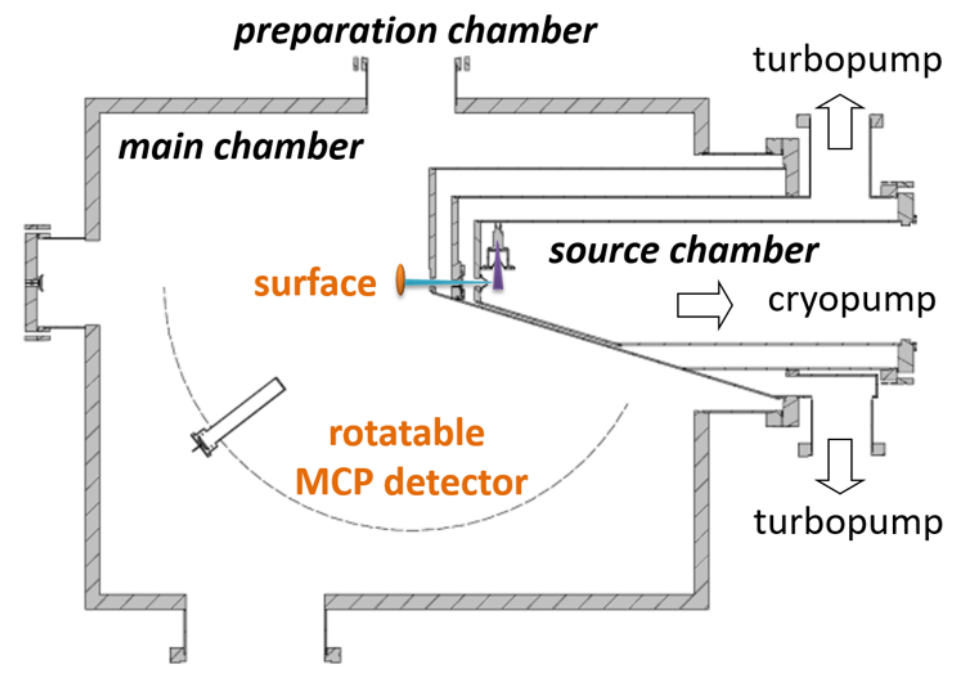

Figure 3.2: Sketch of the RAT scattering machine. After bake out for approximately three days at $120^{\circ} \mathrm{C}$, the base pressure in the preparation, main, and source chamber is in the order of $10^{-10}, 10^{-10}$, and $10^{-8}$ mbar, respectively.

and characterized in the preparation chamber. A standard Ar-ion gun, leak valve, electron bombardment heater, LEED and Auger electron spectrometer is available for surface cleaning, preparation and characterization (for details see Chapter 3.7).

The sample can subsequently be transferred from the preparation chamber into the main chamber. In the main chamber the scattering process and $\mathrm{H}$ atom detection via HRTOF (Section 3.3 takes place. Both chambers are separated from one another by a general valve.

In the high vacuum source chamber the hydrogen atom beam is generated (Section 3.2). It is connected to the main chamber via two differential pumping stages. In general, all chambers are pumped by turbo molecular pumps. During the experiment, the pressure in the source chamber increases from $10^{-8}$ to $10^{-5}$ mbar, pumped by a cryogenic pump to increase pump capacity and reduce corrosion by the applied hydrogen halides. The pressure can be further reduced to $\leq 10^{-6}$ mbar, to decrease surface contamination by hydrogen halides in the main chamber, by application of a liquid nitrogen cooled copper plate underneath the nozzle output. 


\subsection{Generation of well-defined H/D-atom beams}

In this thesis, a neutral hydrogen and deuterium atom beam with a narrow translational kinetic energy distribution was generated solely by photolysis of hydrogen and deuterium iodide molecules via one UV-photon excitation. Figure 3.3 shows the relevant potential energy curves of hydrogen iodide. 91 The red arrows mark the excitation range to which

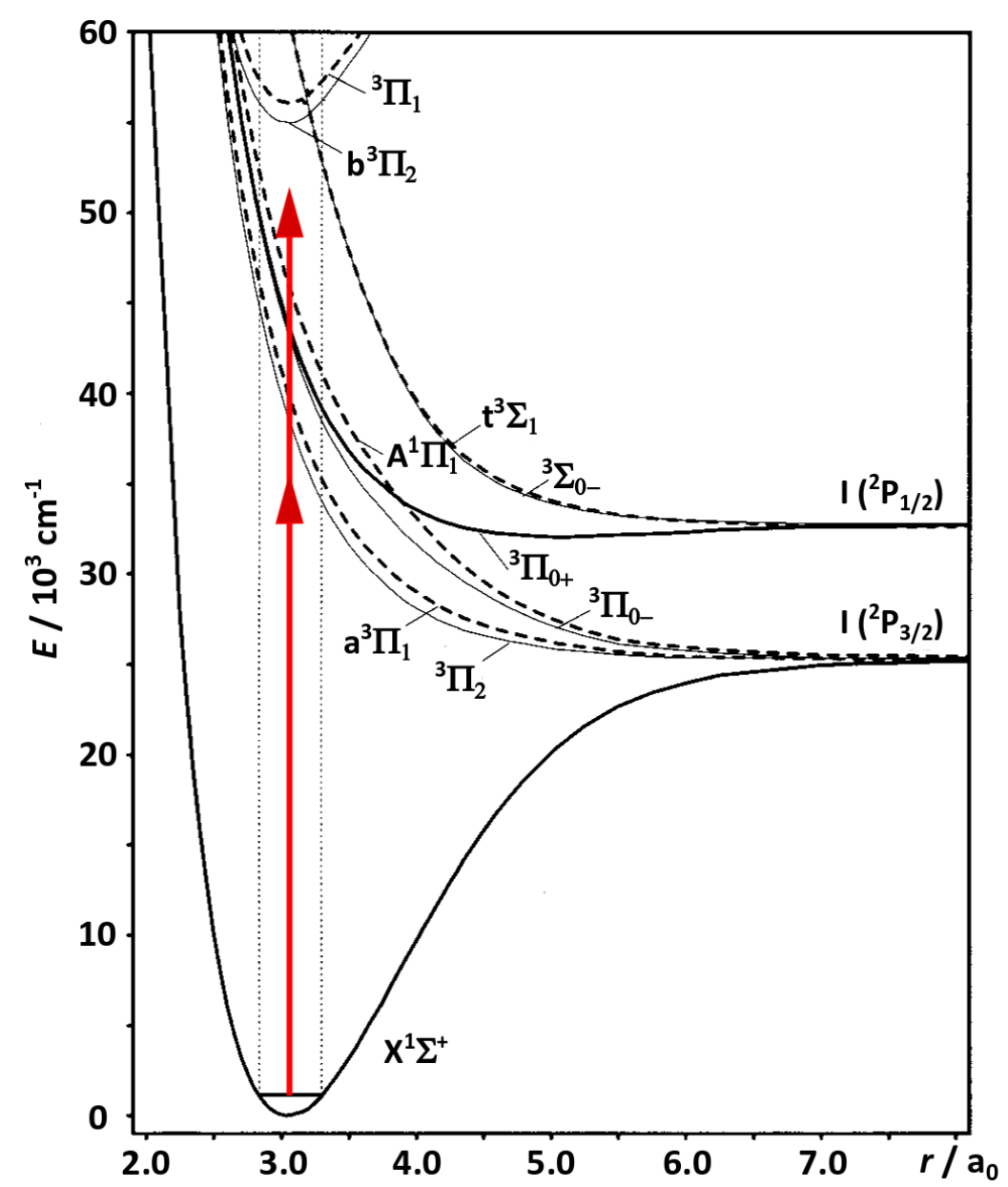

Figure 3.3: Calculated potential energy curves of HI.91 Electronic transitions from the ground state to excited states highlighted by dashed curves are allowed. The red arrows mark the photodissociation wavelengths range used in this thesis. The dashed vertical lines indicate the Frank-Condon region.

the ground state hydrogen halide molecules are excited in the present work. Transitions from the ground state $\mathrm{X}^{1} \Sigma^{+}$to the repulsive states ${ }^{1} \Pi_{1},{ }^{3} \Pi_{1},{ }^{3} \Pi_{0+}$ can occur in the Franck-Condon region (dashed vertical lines). Ground state transitions to the two $\left(0^{-}\right)$ states and the ${ }^{3} \Pi_{2}$ states are forbidden, due to the electric-dipole selection rules. 
The excitation to all repulsive states leads to the dissociation of the molecule into neutral atoms. The possible photodissociation channels for the processes are:

$$
\begin{array}{lll}
\text { 1. } \mathrm{HI}+\mathrm{h} \nu \longrightarrow \mathrm{H}\left({ }^{2} \mathrm{~S}\right)+\mathrm{I}\left({ }^{2} \mathrm{P}_{3 / 2}\right) & \Rightarrow[\mathrm{I}] \\
\text { 2. } \mathrm{HI}+\mathrm{h} \nu \longrightarrow \mathrm{H}\left({ }^{2} \mathrm{~S}\right)+\mathrm{I}\left({ }^{2} \mathrm{P}_{1 / 2}\right) & \Rightarrow\left[\mathrm{I}^{*}\right]
\end{array}
$$

From this point onwards, the halogen atom spin-orbit states are referred to as I and I* with respect to the ground state $\mathrm{I}\left({ }^{2} \mathrm{P}_{3 / 2}\right)$ and spin-orbit excited state $\mathrm{I}\left({ }^{2} \mathrm{P}_{1 / 2}\right)$, respectively. $\mathrm{HI}$ is excited to the repulsive state ${ }^{3} \Pi_{0+}$ via a parallel transition $(\Delta \Omega=0)$ and dissociates adiabatically to $I^{*}$, while it is excited via a perpendicular transition $(\Delta \Omega= \pm 1)$ to the ${ }^{1} \Pi_{1}$ and ${ }^{3} \Pi_{1}$ states which correlate with the ground state I channel. When the transition dipole moment $\vec{\mu}$ is aligned parallel to the electric vector of linear polarized laser light, the absorption probability of the photon is at its maximum and a strong anisotropic angular distribution of the product fragments is observed. For example, assuming a parallel transition $(\Delta \Omega=0$, within Hund's case (a) or (c)) with a transition dipole moment parallel to the molecular axis, molecules are predominantly excited that are aligned parallel to the electric vector of the light. Therefore the fragments primarily fly apart along this direction. For direct dissociation, the resulting fragments have a $\cos ^{2} \theta$ dependent distribution.

In a perpendicular transition $(\Delta \Omega= \pm 1)$ the fragments recoil from one another perpendicular to the electric vector and $a \sin ^{2} \theta$ distribution can be expected.

The following equation gives the general expression for the product angular distribution for a one-photon excitation dissociative process which depends on the angle $\theta$ between the laser polarization axis and recoil velocity vector:92

$$
I(\theta)=\frac{1}{4}\left[1+\beta \cdot P_{2}(\cos \theta)\right]
$$

$I$ denotes the intensity. The second order Legendre polynomial $P_{2}(\cos \theta)$ is given by:

$$
P_{2}(\cos \theta)=\frac{3}{2} \cos ^{2} \theta-\frac{1}{2}
$$

The energy dependent anisotropy parameter $\beta$ adopts values between: $\beta=-1$, for solely perpendicular transitions; and $\beta=+2$, for a purely and immediate parallel transition. An isotropic distribution occurs for $\beta=0$. If $\beta \neq 0$, the main dissociation trajectory of the HI molecule can be controlled with linear polarized laser light, thus, a significant fraction of the atomic hydrogen product fly into the desired direction.

The I* branching fraction and dissociation product angular distribution for UV excitation 
of HI were previously determined by Langford et al. 93

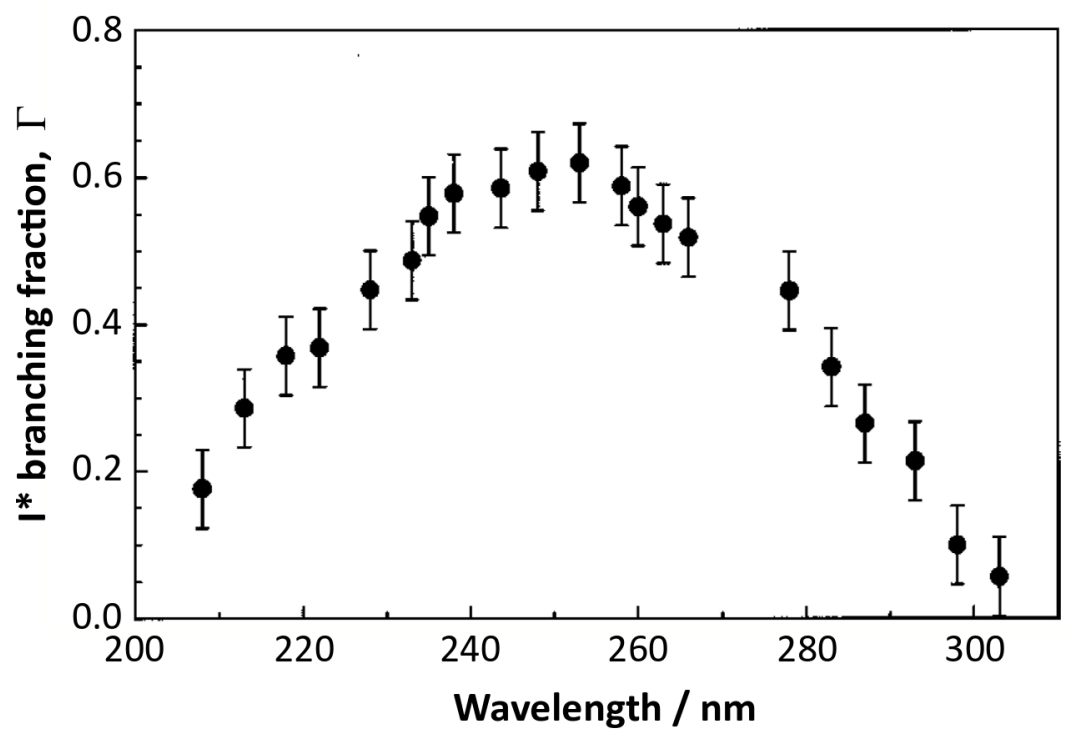

Figure 3.4: Experimentally observed $I^{*}$ branching fraction $\Gamma$ plotted as a function of excitation wavelength from Langford et al.94

Fig. 3.4 shows the measured $I^{*}$ branching fraction $\Gamma$ with respect to the applied UV excitation wavelength. The branching fraction shows a peak maximum at an excitation wavelength of approximately $250 \mathrm{~nm}$. The kinetic energy of the produced hydrogen atoms depends on the dissociation channel. The intensity ratio of the beam of hydrogen atoms from I* and I co-fragments equals the measured I* branching fraction in dependence of the excitation wavelength.

The hydrogen atom beam is generated perpendicular to the HI beam. For the fission of a hydrogen halide molecule (HX), conservation of energy requires the available energy to be distributed among the translational and internal degrees of freedom of the product fragments $\mathrm{H}$ and $\mathrm{X}$.

$$
\mathrm{h} \nu+E_{\mathrm{kin}}^{H X}+E_{\mathrm{int}}^{H X}-D_{0}(\mathrm{HX})=E_{\mathrm{kin}}^{\mathrm{H}}+E_{\mathrm{kin}}^{\mathrm{X}}+E_{\mathrm{int}}^{\mathrm{H}}+E_{\mathrm{int}}^{\mathrm{X}}
$$

Here, $\mathrm{h} \nu$ is the photon energy, $D_{0}(\mathrm{HX})$ is the bond dissociation energy of $\mathrm{H}-\mathrm{X}, E_{\text {int }}$ is the internal energy of the parent molecule HX or the photofragments $\mathrm{H}$ or X, respectively. $E_{\text {kin }}=\frac{1}{2} m \cdot v^{2}$ is the translational kinetic energy of a fragment and is a function of the velocity $v$ and mass $m$ of the respective fragment. Supersonic beam expansion is applied to form a coherent jet of the source HI/DI molecules. This expansion method leads to internally cold molecules, i.e. the rotational and frequently the vibrational temperatures 
of the expanded hydrogen halide molecules HX are very low $\left(E_{\text {int }}^{H X} \approx 0 \mathrm{eV}\right) .9596$ The dissociation process yields the atomic halogen fragment either in its ground state $\mathrm{X}$ or in its excited spin-orbit state $\mathrm{X}^{*}$ with $E_{\mathrm{int}}^{\mathrm{X}}$. The $\mathrm{H}$ atom cannot be excited to a higher energy level, because the available energy of the dissociation process is smaller than the energy required to excite the hydrogen atom (Section 3.3 . Fig. 3.6 from the ground state to any excited state. The initial kinetic energy of the HX molecule can be neglected as only the speed distribution of the products perpendicular to the molecular beam need to be considered for the experiment (Fig. 3.9). In the centre-of-mass frame, the momentum conservation relates the velocity vector of the product fragments to each other as follows:

$$
m_{\mathrm{X}} \cdot{\overrightarrow{v^{\prime}}}_{\mathrm{X}}+m_{\mathrm{H}} \cdot{\overrightarrow{v^{\prime}}}_{\mathrm{H}}=0
$$

Momentum conservation and simplification of equation 3.3 leads to the following expression for the incident hydrogen atoms in the centre-of-mass frame:

$$
E_{\text {kin }}^{\prime \mathrm{H}}=\frac{\mathrm{h} \nu-D_{0}(\mathrm{HX})-E_{\mathrm{inc}}^{\mathrm{X}}}{1+\frac{m_{\mathrm{H}}}{m_{\mathrm{X}}}}
$$

The effective velocity $v_{\mathrm{H}}$ and the derived effective kinetic energy of the hydrogen atom beam are dependent on the momentum of the HX beam. The $\mathrm{H}$ atoms fly perpendicular to the HX beam, thus, the effective velocity of the hydrogen atoms $v_{\mathrm{H}}$ is:

$$
v_{\mathrm{H}}^{2}=v_{\mathrm{H}}^{\prime 2}-v_{\mathrm{HX}}^{2}
$$

Conversion of $E_{\text {kin }}^{\prime \mathrm{H}}$ with $v_{\mathrm{H}}^{\prime}$ from the centre-of-mass frame into the laboratory frame yields:

$$
E_{\mathrm{kin}}^{\mathrm{H}}=\frac{1}{2} m_{\mathrm{H}} v_{\mathrm{H}}^{\prime 2}-\frac{1}{2} m_{\mathrm{H}} v_{\mathrm{HX}}^{2}=\frac{\mathrm{h} \nu-D_{0}(\mathrm{HX})-E_{\mathrm{inc}}^{\mathrm{X}}}{1+\frac{m_{\mathrm{H}}}{m_{\mathrm{X}}}}-\frac{1}{2} m_{\mathrm{H}} v_{\mathrm{HX}}^{2}
$$

The average beam velocity of the HX molecule, with the mass $m_{\mathrm{HX}}$ and the isobaric heat capacity $C_{\mathrm{p}} \approx \frac{7}{2} \mathrm{R}$ can be approximated by equation 3.8 . The velocity depends on the nozzle temperature $\left(T_{\exp }=35^{\circ} \mathrm{C}\right.$ yields $\bar{v}_{\mathrm{HI}}=374 \mathrm{~m} / \mathrm{s}$ and $\bar{v}_{\mathrm{DI}}=373 \mathrm{~m} / \mathrm{s}$, respectively):

$$
\bar{v}_{\mathrm{HX}}=\sqrt{\frac{2 C_{\mathrm{p}} T_{\mathrm{exp}}}{m_{\mathrm{HX}}}}
$$

The first UV absorption bands of hydrogen and deuterium iodide are both broad and featureless, due to the predominance of direct dissociation from repulsive potential energy (PE) surfaces. 93 Therefore, the translational energy of the atomic beam can be easily tuned by the applied UV photon frequency $\mathrm{h} \nu$. 


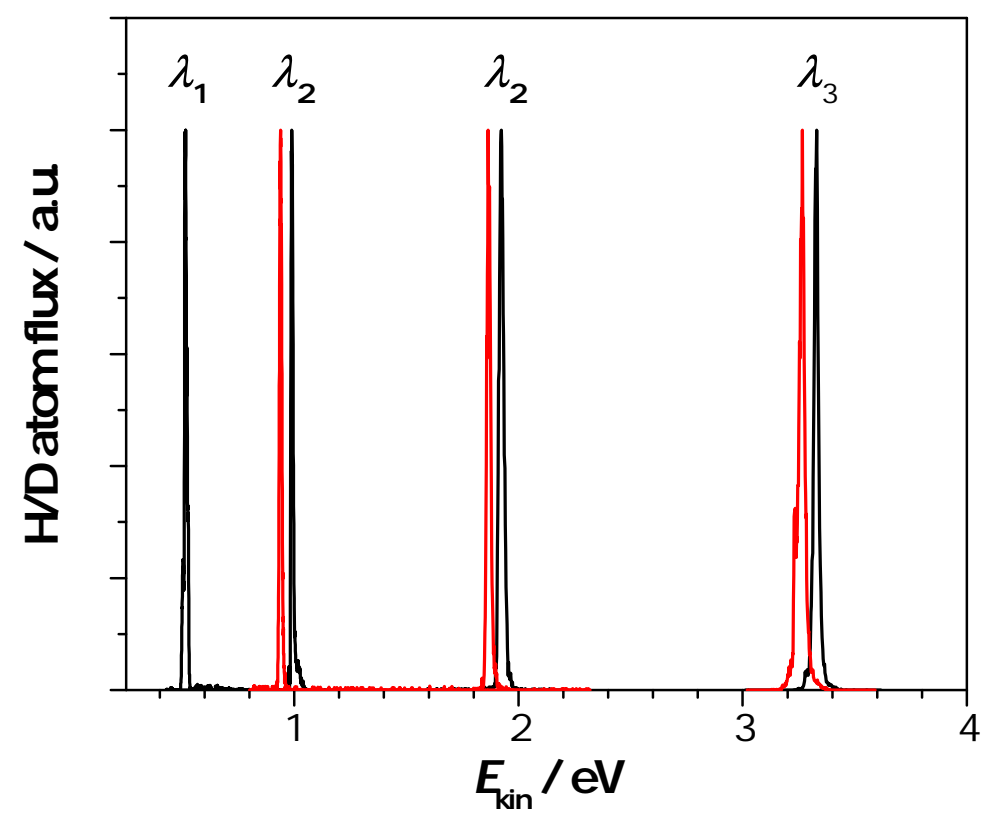

Figure 3.5: Translational energy distribution of the incident $H$ atom (black) and $D$ atom (red) beams with respect to the photolysis wavelength. Hydrogen atom beams with kinetic energies of $E_{\text {kin }}^{\mathrm{H}}=0.51,0.99,1.92$, and $3.33 \mathrm{eV}$, and deuterium atom beams with $E_{\text {kin }}^{\mathrm{D}}=0.94,1.87$, and $3.27 \mathrm{eV}$ were generated with the laser wavelengths $274.8\left(\lambda_{1}\right), 248.35\left(\lambda_{2}\right)$, and $193.3 \mathrm{~nm}\left(\lambda_{3}\right)$.

The kinetic energy of the incident $\mathrm{H} / \mathrm{D}$ atom beam resulting from HI/DI photodissociation can be calculated from equation 3.7 with $D_{0}(\mathrm{HI})=24633 \mathrm{~cm}^{-1}, D_{0}(\mathrm{DI})=24962 \mathrm{~cm}^{-1}$, and $E_{\mathrm{inc}}^{\mathrm{X}}=7603 \mathrm{~cm}^{-1} .9798$

Figure 3.5 shows the kinetic energy distribution for the incident $\mathrm{H}$ (black) and D atom beams (red) used in this thesis. Hydrogen atom beams with kinetic energies of $0.51-3.33 \mathrm{eV}$ and deuterium atom beams with kinetic energies of $0.94-3.27 \mathrm{eV}$ were generated. The full width at half maximum (FWHM) of the incidence beams and hence the kinetic energy resolution are in the range of $10-20 \mathrm{meV}$ and depend primarily on the wavelength bandwidth and focus size of the applied photolysis laser. 


\subsection{Rydberg atom tagging time-of-flight}

By means of the $\mathrm{H}$ atom Rydberg tagging time-of-flight technique, a TOF spectrum of the neutral scattered hydrogen atoms can be measured with a very high translational energy resolution.

A detailed description of the HRTOF technique can be found in literature, 99 while a brief description will be provided here. The technique is characterized by the excitation of the $\mathrm{H}$ atoms to a high $n$ Rydberg state $(n \approx 30-90)$ close to the ionization threshold. 100 In such highly excited Rydberg states, the angular momentum $l$ can occupy high values $(l \leq n-1)$. Particularly for such high angular momentum values, the probability overlap of the wave functions of $l$ states with the wave functions of the electrons in low energy levels is very low. Thus, the spontaneous relaxation of an electron in the Rydberg state to the ground state becomes highly improbable yielding a dramatically increased lifetime for these states. Furthermore, the population of Rydberg atoms in high $l$ states can be further increased in low electric fields. 101,102 Previous experiments indicated, that in a low electric field $(\approx 10 \mathrm{~V} / \mathrm{cm})$ hydrogen atoms in high Rydberg states $(n \approx 50)$ have lifetimes on a millisecond time-scale. 90

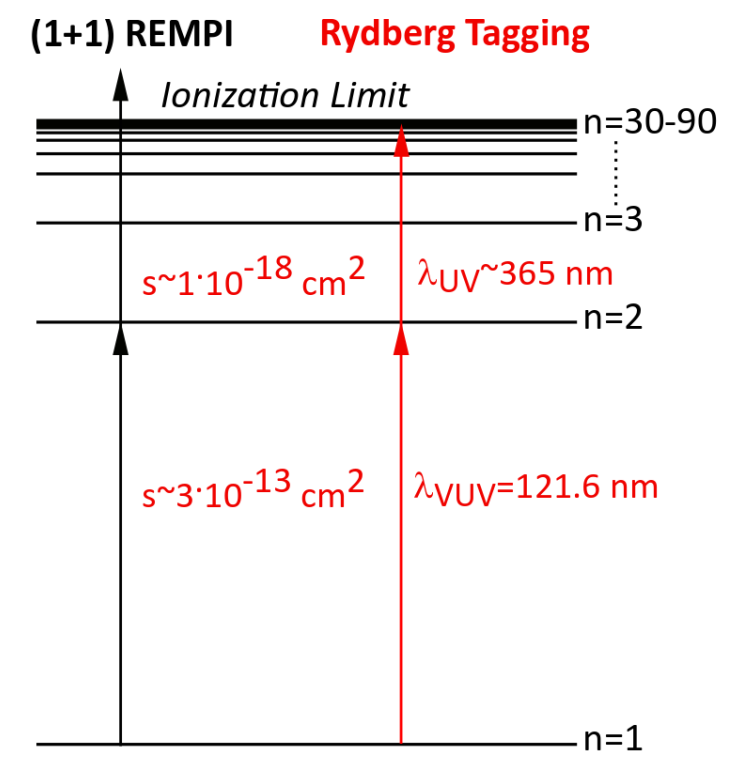

Figure 3.6: Detection schemes for hydrogen atoms. The Rydberg tagging technique excites an $\mathrm{H}$ atom to a high $n$ Rydberg state close to the ionization threshold $(n=30-90)$. The $(1+1$ ')-REMPI technique directly ionizes the $\mathrm{H}$ atoms. 103

In order to promote scattered hydrogen atoms to a high Rydberg state, a two-step excitation of the hydrogen atoms from the ground state to a high Rydberg state can 
be applied (Fig. 3.6, right excitation scheme). In the first step, the $\mathrm{H}$ atom is excited from the ground state $n=1$ to the $n=2$ state using laser light of Lyman $\alpha$ wavelength $\left(\lambda_{\alpha}=121.6 \mathrm{~nm}\right)$. This excitation can be easily saturated, as this particular transition possesses a high excitation cross-section. The second excitation step promotes the $\mathrm{H}$ atom from the $n=2$ level up to a high Rydberg state (in this thesis: $n=34$ ) using $365 \mathrm{~nm}$ laser radiation. The neutral Rydberg hydrogen atoms then fly a well-defined distance to the ion detector, where a small electric field ionizes the tagged $\mathrm{H}$ atom prior to their detection. The obtained translational energy resolution depends on the physical size of the tagging region and the field ionization area. Investigations showed that energy levels of the Rydberg $\mathrm{H}$ atom in ranges of $n=30-90$ have no significant effect on the TOF translational distribution. 90

Another commonly applied technique is REMPI (resonance-enhanced multi-photon ionization), which directly ionizes the hydrogen atoms. However, in comparison the Rydberg atom tagging method has a significantly improved flight-time resolution compared to the REMPI technique, as the atoms are neutral until they reach the detector. This advantage stems from the fact that due to their neutral charge, these atoms and thus their flight-time resolution are neither affected by space charge effects nor stray fields. 


\subsection{Laser system}

In this section the laser set-up used in the experiment is described (Fig. 3.7), which can be divided into two different systems. First, the photolysis laser system creates a nearly mono-energetic hydrogen atom beam by photodissociation of hydrogen halides by using dissociation wavelengths of 193.3, 212.56, 248.35 and $274.8 \mathrm{~nm}$. Second, the tagging laser system excites the scattered hydrogen atoms to a high $n$ Rydberg state $(n=34)$ via a two-step excitation (Section 3.3). This process requires two laser beams with wavelengths of $121.6 \mathrm{~nm}$ and $365 \mathrm{~nm}$. The VUV $121.6 \mathrm{~nm}$ laser light is generated by resonance-enhanced third-order difference four-wave mixing of $212.56 \mathrm{~nm}$ and $845 \mathrm{~nm}$ in a krypton gas cell.

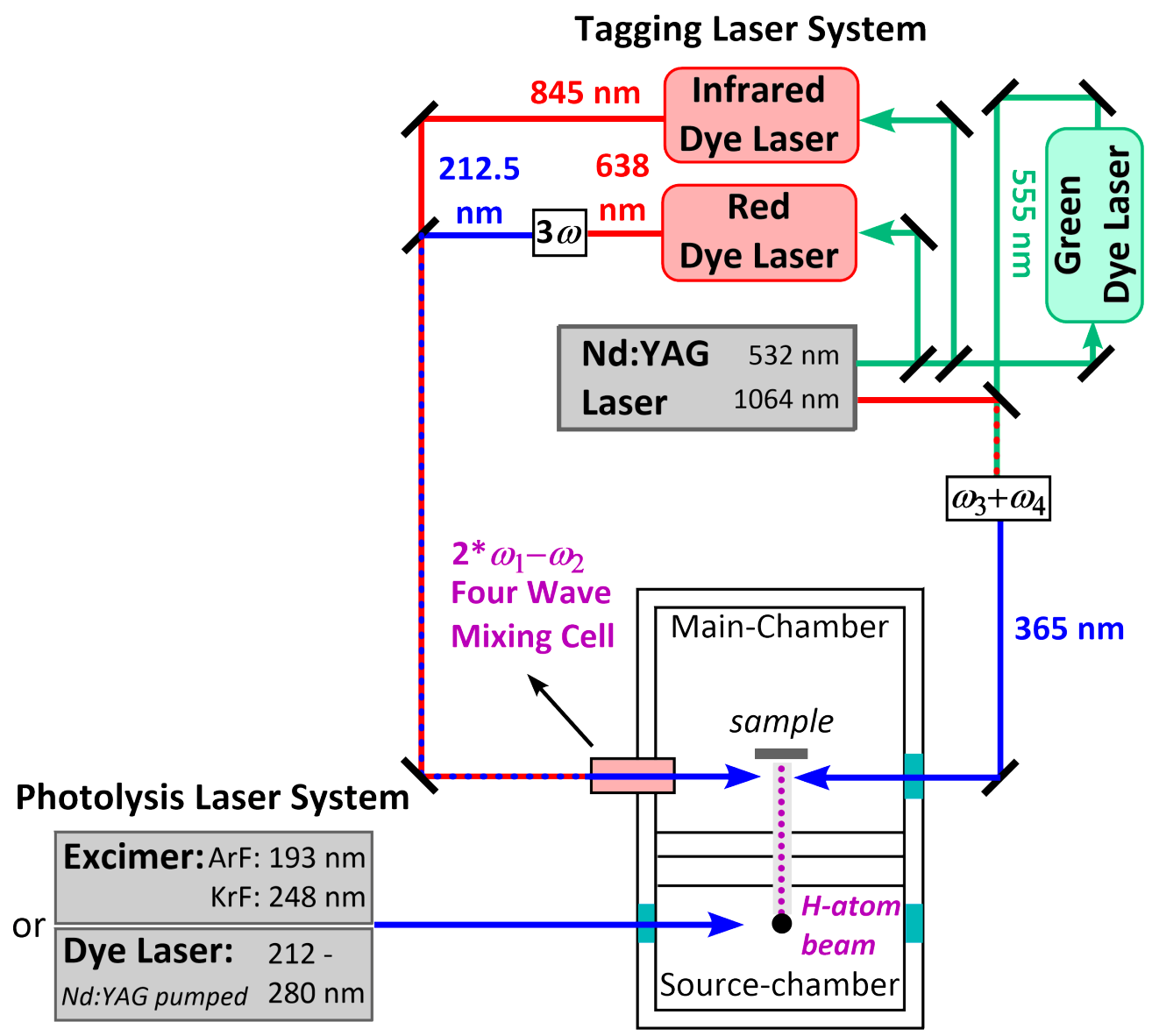

Figure 3.7: Schematic arrangement of the laser system. 


\subsubsection{Laser set-up}

\section{Tagging laser system}

The tagging laser system consists of three dye lasers pumped by the second harmonic (532 nm) of a Nd:YAG (yttrium aluminum garnet) laser (Spectra Physics, Quanta Ray PRO-250-30, $1064 \mathrm{~nm}$ ) with a repetition rate of $30 \mathrm{~Hz}$ and a pulse length of $\sim 10 \mathrm{~ns}$. The red dye laser (Sirah Precision Scan) pumped by $140 \mathrm{~mJ}$ of $532 \mathrm{~nm}$ generates approximately $60 \mathrm{~mJ}$ of $638 \mathrm{~nm}$ laser light (laser dye: DCM dissolved in ethanol). The $638 \mathrm{~nm}$ laser output is frequency doubled in a non-linear barium borate (BBO) crystal and subsequently frequency mixed with the fundamental $638 \mathrm{~nm}$ in a second $\mathrm{BBO}$ crystal to yield $2-3 \mathrm{~mJ}$ of $212.56 \mathrm{~nm}$ UV radiation. The infrared (IR) dye laser is pumped by $130 \mathrm{~mJ}$ of the second ordered harmonic laser light in order to generate $845 \mathrm{~nm}$ laser radiation $(15 \mathrm{~mJ})$. The laser dye consists of a dye mixture of LDS821 and LDS827 dissolved in ethanol. The $212.56 \mathrm{~nm}$ and $845 \mathrm{~nm}$ laser beams are aligned within a four-wave mixing cell to produce Lyman $\alpha 121.6 \mathrm{~nm}$ VUV laser radiation. The mixing cell is covered at the entrance and exit with a quartz window and a LiF lens, respectively, in order to focus the VUV laser light towards the tagging region. The green dye laser (Sirah Cobra Stretch, pumped by $\sim 130 \mathrm{~mJ}$ of $532 \mathrm{~nm}$ ) produces $555 \mathrm{~nm}$ laser radiation (laser power: $\sim 25 \mathrm{~mJ}$, laser dye: pyrromethene 580 in ethanol). The $555 \mathrm{~nm}$ laser light is frequency mixed in a BBO crystal with the fundamental laser output of the Nd:YAG laser $(1064 \mathrm{~nm}, 400 \mathrm{~mJ})$ in order to produce $365 \mathrm{~nm}(\sim 12 \mathrm{~mJ})$ for the second Rydberg tagging step. The $365 \mathrm{~nm}$ laser beam is co-aligned with the $212.56 \mathrm{~nm}$ and $845 \mathrm{~nm}$ laser beam and focused within the tagging region.

\section{Photolysis laser system}

The photolysis laser system consists of an excimer (Coherend, COMPexPRO 205) or alternatively a tunable dye laser (Sirah Precision Scan) pumped by a second Nd:YAG laser (200 mJ, Spectra Physics, Quanta Ray PRO-290-30, repetition rate $30 \mathrm{~Hz}$, pulse length $10 \mathrm{~ns}$ ). The excimer laser can operate at a wavelength of either $193.3 \mathrm{~nm}$ or $248.35 \mathrm{~nm}$ depending on the utilized gas filling. A gas filling with ArF gives access to $193.3 \mathrm{~nm}(\sim 150 \mathrm{~mJ}$, bandwidth $\sim 1 \mathrm{~nm})$, whereas $\mathrm{KrF}$ emits $248.35 \mathrm{~nm}(\sim 250 \mathrm{~mJ}$, bandwidth $\sim 0.5 \mathrm{~nm})$. The bandwidth of the excimer laser depends on the discharge voltage and gas mixture fill (pressure and type of buffer gas).

The excimer laser has the advantage that it produces UV laser light with extremely high energy density, resulting in a substantially more intense hydrogen atom beam. However, 
the wavelength of the laser output is fixed, the beam is unpolarized and has a relatively broad bandwidth $(\Delta \lambda=0.5-1 \mathrm{~nm})$.

The laser output of the dye laser is linearly polarized, has a tunable wavelength and a much narrower bandwidth $(0.004 \mathrm{~nm})$. By varying the laser dye and by using frequency doubling or mixing crystals, UV laser wavelengths of $212.56 \mathrm{~nm}$ and $274.8 \mathrm{~nm}$ were generated. The $212.56 \mathrm{~nm}$ can be created by the same procedure as described before in the tagging laser system. In order to produce $274.8 \mathrm{~nm}$, the laser dye is changed to pyrromethene 580 in ethanol. The $550 \mathrm{~nm}$ laser output is frequency doubled giving access to $15 \mathrm{~mJ}$ of $274.8 \mathrm{~nm}$ light. The UV radiation is adjusted within the chamber in order to intersect the molecular beam in the photodissociation region. By utilization of a rotatable half waveplate, the polarization of the laser beam can be rotated and optimized for the purpose of generating a hydrogen atom beam predominantly in the desired direction.

\subsubsection{VUV generation}

The application of difference frequency mixing in a non-linear optical media for the generation of coherent tunable narrow-bandwidth VUV radiation in high intensities made it possible to study various atomic and molecular processes. 104 Noble gases (Xe, Kr, Ar, Ne and $\mathrm{He}$ ) are generally used as nonlinear media due to their suitable energy level structures and convenient handling. Equation 3.9 describes the general mathematical relationship of the resonance-enhanced third-order difference four-wave mixing (DFWM) applied in the present work.

$$
\omega_{\mathrm{VUV}}=2 \cdot \omega_{1}-\omega_{2}
$$

The VUV $121.6 \mathrm{~nm}$ laser beam $\omega_{\mathrm{VUV}}$ used in the first step of the hydrogen atom excitation, can be generated by DFWM of two $\omega_{1}$ photons $\left(\lambda_{1}=212.56 \mathrm{~nm}\right)$ and one $845 \mathrm{~nm}$ photon $\omega_{2}$ in a krypton gas cell. Figure 3.8 illustrates the general four wave mixing scheme in Kr. The $4 \mathrm{p}^{5} 5 \mathrm{p}$ state is resonant with a two photon transition of the frequency $\omega_{1}\left(\lambda_{1}=212.56 \mathrm{~nm}\right)$. By inducing one infrared photon of the frequency $\omega_{2}\left(\lambda_{2}=846 \mathrm{~nm}\right)$, krypton reverts to the ground state emitting light of the Lyman $\alpha$ wavelength $\left(\lambda_{\alpha}=121.6 \mathrm{~nm}\right)$. In order to achieve an efficient transition process, the waves involved have to be colinearly aligned. In eq. 3.10 the wave vector $\vec{k}_{i}$ corresponds to the phase velocity of the involved waves.

$$
\Delta k=\vec{k}_{\mathrm{VUV}}-\left(2 \cdot \vec{k}_{1}-\vec{k}_{2}\right)=0
$$




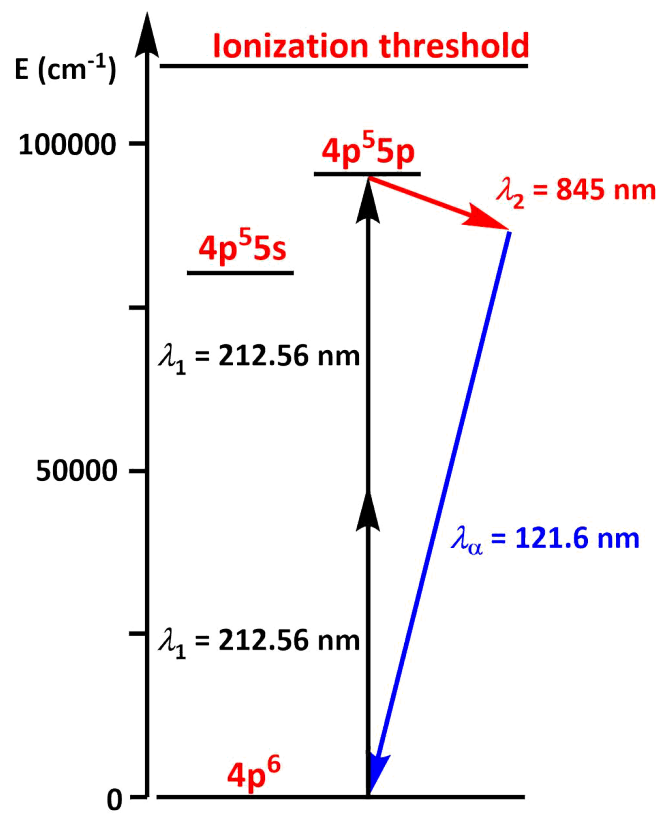

Figure 3.8: Resonance-enhanced difference four-wave mixing scheme to generate VUV radiation at $\lambda_{\alpha}=121.6 \mathrm{~nm}$ in a $\mathrm{Kr}$ gas cell. 105

The dispersion of the phase velocities have to equal zero, therefore, the phase velocities of the different wavelengths have to be adjusted with respect to each other. One possibility for the adjustment is to vary the krypton pressure in the mixing cell as has been previously reported.105 106 However, this effect is limited. In order to achieve a higher efficiency, a gas mixture can be used with a gas of negative and a gas of positive dispersion. 107 Argon has positive dispersion in the wavelength range from 121.1 to $180 \mathrm{~nm}$, while krypton is a negative dispersing gas up to $123.6 \mathrm{~nm}$. The $121.56 \mathrm{~nm}$ wavelength can be generated by a mixture of argon and krypton (pressure ratio 3:1) in a clear stainless steel gas cell. By recording the intensity of the hydrogen atom signal against the gas cell pressure, an optimal pressure of the Ar/Kr-mixture was found to be at $100-120$ mbar. 


\subsection{Experimental set-up, data acquisition and processing}

An overview of the experimental set-up is illustrates in Fig. 3.9 and a short summary of the experiment will be given.

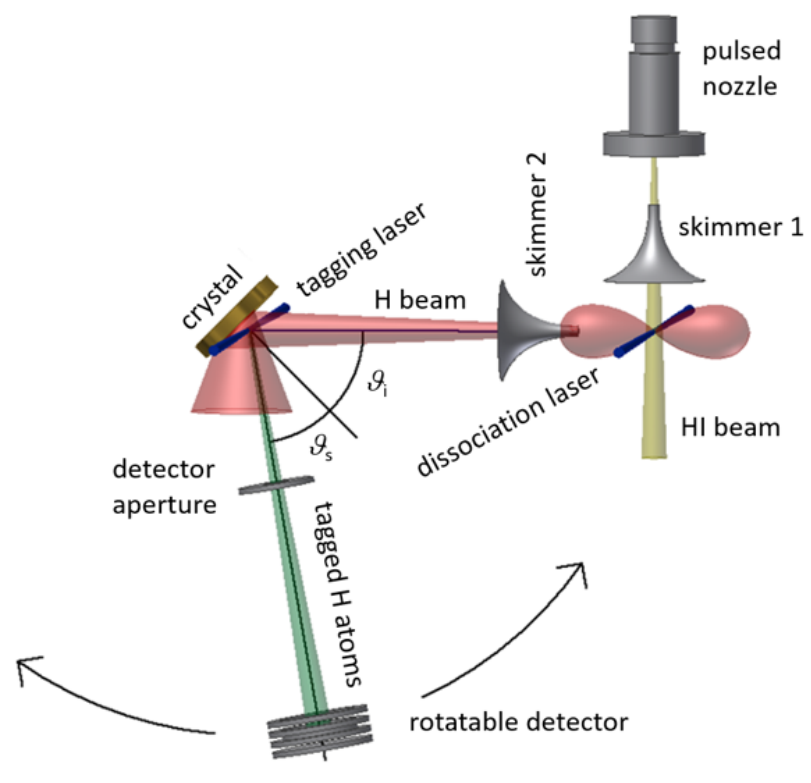

Figure 3.9: Detailed geometry of the experimental set-up. 28

A rotationally cold hydrogen halide molecular beam is formed in the source chamber by supersonic expansion through a pulsed nozzle with a back pressure of 6 bar. The hydrogen halide beam passes skimmer 1 and crosses the UV photodissociation region. The skimmer prevents molecules with velocities that are not aligned parallel to the molecular beam from flying into the photodissociation area. The fraction of the $\mathrm{H}$ atom photoproduct, which is dissociated in the direction of the main chamber, passes skimmer 2 and two differential pumping apertures to enter the UHV-scattering chamber. Here, the $\mathrm{H}$ atom beam collides with the sample mounted on a 6-axis manipulator. The sample mount allows the polar $\vartheta_{\mathrm{i}}$ angle and azimuthal angle $\varphi_{\mathrm{i}}$ of incidence to be varied. The angle of incidence and polar scattering angle $\vartheta_{\mathrm{s}}$ are defined with respect to the surface normal. At a distance of $0.7 \mathrm{~nm}$ from the surface, the scattered $\mathrm{H}$ atoms are detected by HRTOF. All neutral Rydberg atoms with flight direction perpendicular to the laser beams, travel $\sim 255 \mathrm{~nm}$, pass through a grounded mesh and are field-ionized by a moderate electric field. These ions are detected using a micro-channel plate (MCP) detector. The amplified MCP signal is converted and recorded by a discriminator and a multichannel scalar. The detector is rotatable within a range of $40-180^{\circ}$ with respect to the incidence beam, thus, 
allowing TOF distributions to be measured for various scattering angles $\vartheta_{\mathrm{s}}$ in the plane of the incidence beam. The TOF distribution and angular distribution of the scattered $\mathrm{H} / \mathrm{D}$ atoms are measured in relation to the incidence energy, incidence angle and scattering angle. The angular resolution of the detector $\left(3^{\circ}\right)$ is defined by the size of the aperture $150 \mathrm{~mm}$ in front of the detector.

In order to successfully perform the experiment, the pulsed molecular beam and the photodissociation laser as well as the scattered atom beam and the tagging laser have to overlap in space and time. The overlap in space is optimized using laser optics. A SRS DG635 delay generator controls the firing times of the laser and the nozzle opening. The generated molecular beam has to travel for a given period of time to reach the photodissociation point. A time overlap of the molecular beam and the photolysis laser was achieved with a dissociation laser delay of approximately $200-300 \mu$ s compared to the nozzle opening time. The flight time of the atoms from the dissociation point to the tagging point depends on their inherent kinetic energy. The delay between the dissociation and tagging laser varies from $7-17 \mu$ s with respect to the atom beam energy. The measured TOF distribution $g(t) \mathrm{d} t$ can be further converted into the total product translational energy distribution $F(E) \mathrm{d} E$ applying a Jacobian correction. 92 The TOF distribution $g(t) \mathrm{d} t$ represents the number of $\mathrm{H}$ atoms that cross the tagging area in a specific period of time $\mathrm{d} t$. The time dependence on the velocity of the hydrogen atoms, thus, the intensity of the TOF signal is proportional to the particle density. In order to obtain a flux-weighted TOF signal $g_{\text {flux }}(t)$ the density signal $g_{\text {dens }}(t)$ has to be multiplied by the particle velocity $v(t) \propto \frac{1}{t}$.

$$
\begin{aligned}
g_{\text {flux }}(t) & =v(t) \cdot g_{\text {dens }}(t) \\
F(E) \mathrm{d} E & =v(t) \cdot g_{\text {dens }}(t) \mathrm{d} t \quad \text { with } \quad \frac{\mathrm{d} E}{\mathrm{~d} t} \propto \frac{1}{t^{3}} \\
\Rightarrow \quad F(E) & \propto \frac{1}{t} \cdot \frac{\mathrm{d} t}{\mathrm{~d} E} \cdot g_{\text {dens }}(t) \propto t^{2} \cdot g_{\text {dens }}(t)
\end{aligned}
$$

Data handling of the TOF distribution files into energy distribution files is described in detail in Appendix A. In general, translational energy distributions presented in this thesis are normalized to the integral of the distribution. Angular distributions were measured by accumulation of the counting signal for a certain TOF range. Due to the experimental geometry, the detector records different area sizes of the crystal depending on the incidence and scattering angle. A correction of the measured raw angular distribution data is given in Appendix B. All angular distributions presented here are normalized to the peak maximum. 


\subsection{Surface characterization techniques}

\subsubsection{Auger electron spectroscopy (AES)}

Auger electron spectroscopy is a frequently applied method to qualitatively and quantitatively analyze the atomic composition of a given surface. The method is based on the Auger effect, which was first discovered by Lise Meitner 108 and is named after Pierre Auger, who described the effect independently in 1925.109
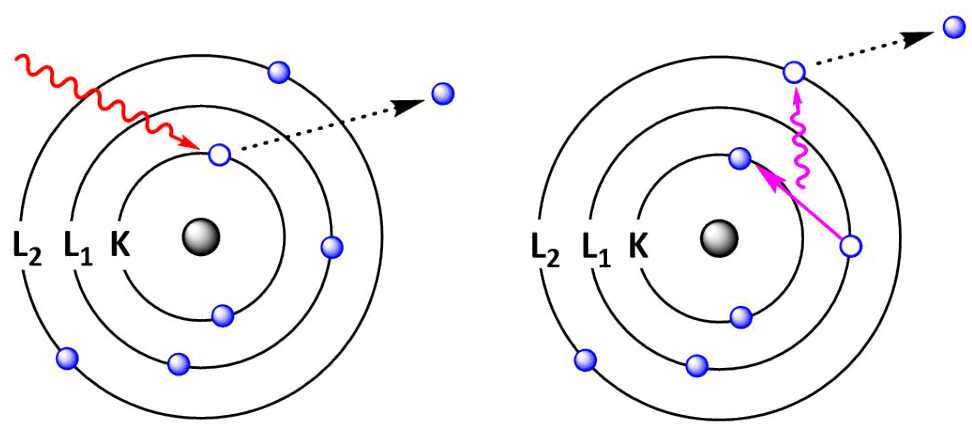

Figure 3.10: A scheme illustrating the electron movement in the Auger process.

The Auger effect relies on the coupling between electrons of different atomic energy levels (Fig 3.10.4 An inner core level electron (e.g. K-shell electron) is ejected by highly energetic photons, electrons or ions. AES uses electron bombardment as an energy source $(3-5 \mathrm{keV})$. In a radiationless transition the produced hole is refilled by an electron of a higher shell $L_{1}$. The excess energy of this electron is instantaneously removed by emission of a higher shell electron $\mathrm{L}_{2}$. The emitted electron is called the Auger electron and is labelled after the shells which are involved in the Auger process. In the described example the resulting Auger electron peak is named KLL.

AES measures the number of electrons $N$ with respect to their kinetic energy $E_{\text {kin }}$. The energy of the Auger electron is strongly dependent on the shells involved and thus characteristic for each element. It can detect almost every element, apart from $\mathrm{H}$ and $\mathrm{He}$ (detection of $\mathrm{H} / \mathrm{He}$ is not possible due to the lack of more than one occupied shell). The kinetic energy of the Auger electron $E_{\text {Auger }}$ can be estimated using the following equation:

$$
E_{\text {kin }} \approx E_{\text {Auger }}=E_{1}-E_{2}-\Phi
$$

Here, $E_{1}$ and $E_{2}$ are the energy levels of the inner core shells (here, $\mathrm{K}$ and $\mathrm{L}_{1}$ ) involved in the Auger process. The difference yields the excess energy, which ejects the Auger electron into the vacuum. To be released into the vacuum, the electron must overcome the work 
function $\Phi$, hence the kinetic energy is reduced by this amount. The kinetic energy of the Auger electron is independent of the primary electron energy. The energy levels of an element and thus the kinetic energy of the Auger electron can be effected by its chemical environment, however, the appearing shifts are generally negligible. Problematic can be the interpretation of Auger spectra of insulating surfaces as on these surface charging can easily occur and drastically influence the kinetic energy of the emitted Auger electrons. $\frac{4}{4}$ Besides the Auger process, a number of other processes occur simultaneously, such as electron backscattering and secondary electron emission. In order to better identify the Auger peaks, the first derivative of the number of electrons as a function of the kinetic energy $\mathrm{d} N / \mathrm{d} E$ is usually plotted. The sensitivity limit of an atomic component on the surface is approximately $1 \%$ with respect to the number of surface atoms.

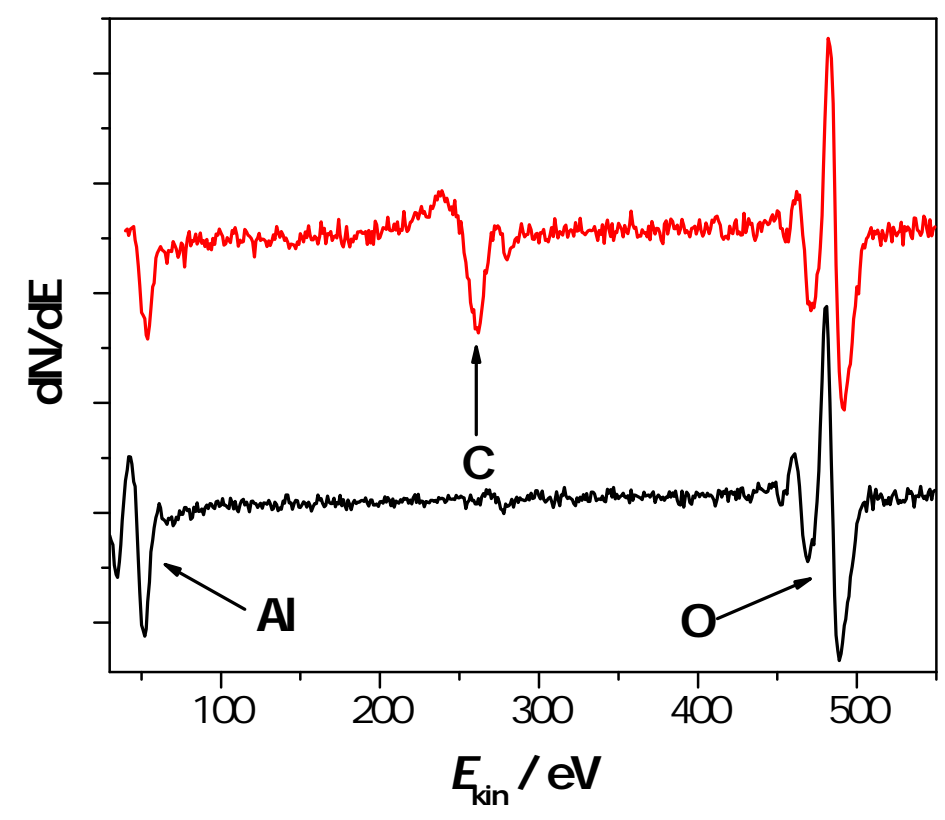

Figure 3.11: Auger spectra of a carbon contaminated (red line) and clean (black line) $\alpha-\mathrm{Al}_{2} \mathrm{O}_{3}$ sample, recorded with a primary electron beam of $3 \mathrm{keV}$. The Auger peaks are assigned to the elements.

An example of an experimentally obtained Auger spectrum of a carbon-contaminated aluminum oxide sample (red line) can be found in Fig 3.11. After annealing for several hours in an oxygen atmosphere $\left(10^{-6}\right.$ mbar) the carbon impurities (Signal around $250 \mathrm{eV}$ ) were removed (black line). The Auger peaks are labeled with the corresponding elements. The elemental surface composition of a sample can be estimated from the peak intensities 
$I$ of the elements. Eq. 3.13 can be used to calculate the mole fraction $C$ of component A.

$$
C_{A}=\frac{I_{\mathrm{A}} / s_{\mathrm{A}}}{\sum_{x} I_{\mathrm{x}} / s_{\mathrm{x}}}
$$

The measured relative sensitivity factor $s$ can be found in the literature.110 As a first approximation the peak intensity of a component can be taken as the peak height in the differential mode. For a more accurate estimation the peak area of the integral mode spectra should be considered.

\subsubsection{Low energy electron diffraction (LEED)}

Low energy electron diffraction is a commonly applied technique for surface structure analysis under UHV conditions. Qualitative LEED can provide information on the unit cell and geometry of the surface. 1111 Therefore, changes to the unit cell by surface reconstruction or adsorbates can be measured on a short timescale.

In general, a low energy electron beam is directed perpendicularly onto a sample. The elastically scattered electrons travel towards a luminescent (phosphor) screen, where the diffraction pattern is monitored using a camera. The wavelength of the incident electron is given by the de Broglie relation:

$$
\lambda=\frac{h}{\sqrt{2 m_{\mathrm{e}} e V}}
$$

Here, $m_{\mathrm{e}}$ is the mass of an electron, $e$ is the electronic charge and $V$ is the beam voltage. Typical electron beam energies of $50-200 \mathrm{~V}$ correspond to electron wavelength between 0.9 and $2.7 \AA$, which is in the same order of magnitude as atomic spacing. The electron mean free path is relatively independent of the investigated material. Due to strong electron-solid interactions, elastically scattered electrons only penetrate within a depth of a few atomic layers $(5-10 \AA) .111$ Thus, the LEED diffraction pattern only provides information on the topmost layers. Nevertheless, lower surface layers influence the diffraction structure, for instance in the spot intensity. 112 The diffraction pattern visualizes the reciprocal lattice of the surface layer from periodic ordered atoms on the surface. In general, a single crystalline surface leads to spot pattern while highly ordered surfaces may create ring patterns and amorphous structures usually do not produce any regular pattern.

Fig. 3.12 (left panel) shows a LEED measurement of an oxygen covered single crystalline 
$f c c \operatorname{Pt}(111)$ surface. The $f c c$ (111) surface cut provides a sixfold symmetry orientation of the surface atoms (see Fig. 3.12). The sixfold symmetry of the $\mathrm{Pt}(111)$ surface is visible in the LEED picture and is highlighted by the red hexagon. The other spots originate from diffraction of the oxygen adsorbates. A $p(2 \times 2)$ orientation of the oxygen atoms on the Pt surface can be derived by converting the LEED pattern of the reciprocal lattice to real space.113 The adsorbed oxygen atoms occupy threefold hollow sites on the platinum surface. 114

Figure 3.12 (right panel) depicts a sketch of the oxygen atom configuration on the $\mathrm{Pt}(111)$ surface in real space. The blue rhombus marks the units cell of the oxygen atom orientation, visualizing a $25 \%$ atomic oxygen coverage of the $\operatorname{Pt}(111)$ surface. The $[10 \overline{1}]$ and $[11 \overline{2}]$ directions of the $\operatorname{Pt}(111)$ surface are illustrated as red arrows.
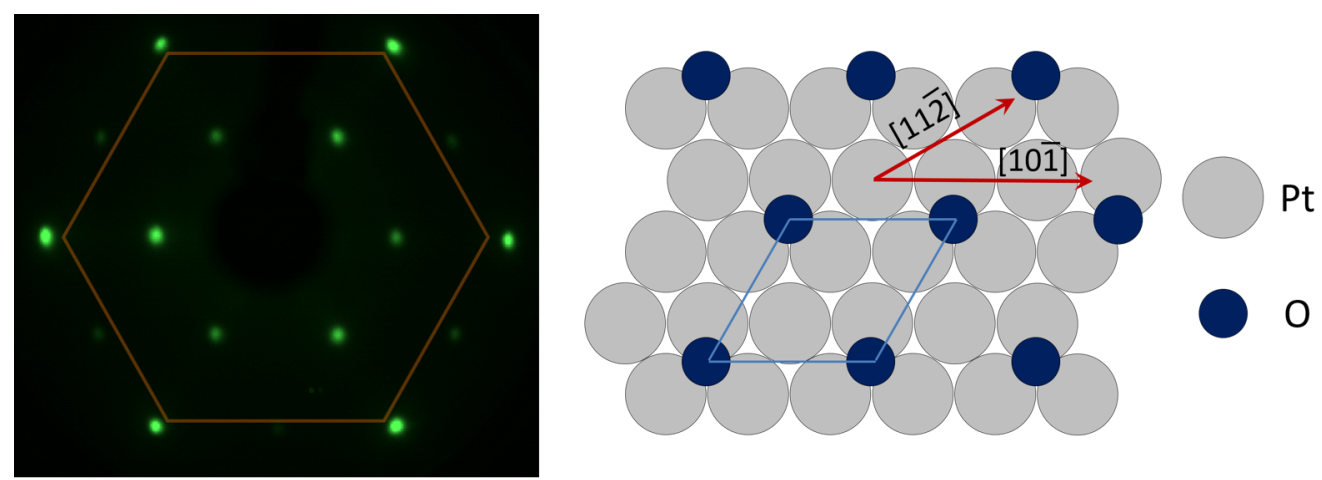

Figure 3.12: Left panel: LEED pattern of a $(2 \times 2)$ atomic oxygen covered $\mathrm{Pt}(111)$ surface, recorded with an electron beam energy of $70 \mathrm{eV}$.

Right panel: Sketch of a $p(2 \times 2)$ atomic oxygen (blue) orientation on a $\operatorname{Pt}(111)$ (grey) surface. The blue rhombus shows the unit cell. The red arrows mark the $[10 \overline{1}]$ and $[11 \overline{2}]$ directions on the $\operatorname{Pt}(111)$ surface, respectively. 


\subsection{Surface preparation and characterization}

In Chapter 4. $\mathrm{H}$ atom scattering from the $f c c$ (face-centered cubic) metals: $\mathrm{Au}, \mathrm{Pt}, \mathrm{Ag}$, $\mathrm{Pd}, \mathrm{Cu}$, and $\mathrm{Ni}$ with a (111) surface cut will be investigated. Chapter 5 studies insulating and ultra-thin layered surfaces. As an insulating surface, a single crystal $\alpha-\mathrm{Al}_{2} \mathrm{O}_{3}$ surface with a (0001) surface cut was studied and compared to ultra-thin films of aluminum oxide. Various ultra-thin layers of aluminum oxide (AlOx) were prepared by atomic layer deposition (ALD): $50 \mathrm{~nm} \mathrm{AlOx}$ on $\mathrm{SiO}_{2}, 1 \mathrm{~nm} \mathrm{AlOx}$ on Pt and a not fully closed monolayer (ML) of AlOx on Pt (prepared by one ALD cycle). The platinum substrates had a thickness of $\sim 50 \mathrm{~nm}$ and were prepared by ALD on a silicon waver. In Chapter 6 , graphene layers generated in situ onto $\mathrm{Ni}(111)$ are examined and compared to a graphene layer on $\mathrm{Pt}(111)$.

In order to perform scattering experiments of atomically clean surfaces, contamination of the investigated samples has to be excluded for several hours. Therefore, the experiments were performed under UHV conditions $\left(10^{-10} \mathrm{mbar}\right)$. As the surfaces were exposed to air prior to their introduction into the RAT machine, the surfaces contained impurities such as adsorbed carbon and water. Additionally, impurities of calcium on platinum and sulfur on silver were observed via AES in the course of the experiments. For the wide variety of investigated samples, different cleaning procedures had to be applied to remove the impurities from the surfaces. In the following sections cleaning procedures and surface synthesis are presented in detail. The surface cleanliness was verified by AES and the surface structure and orientation was determined and verified by LEED. In addition, the scattering signal itself is very sensitive to surface contamination (in particular carbon and hydrogen) and can be used to double-confirm the integrity of a surface.

\subsubsection{Surface cleaning procedure}

All metal surfaces were cleaned by multiple cycles of Ar-ion sputtering followed by high temperature annealing, adapting cleaning procedures from the literature. 115 Argon-ion sputtering removes the atoms of the topmost surface layers. The argon-ion gun operates at $3 \mathrm{kV}$ (Ar background pressure $1 \cdot 10^{-6}$ mbar, ion current $30-40 \mu \mathrm{A}$ ). During each cleaning cycle the samples were sputtered for approx. $30 \mathrm{~min}$. After each sputtering cycle the surfaces were annealed in UHV for 30 min via electron bombardment in order to rebuilt the (111) surface structure. For electron bombardment, a thorium doped tungsten wire is placed closely behind the rear side of the specimen. A current of $2-3 \mathrm{~A}$ on the tungsten filament emits free electrons which can be accelerated towards the sample 
surface by applying a current of positive high voltage $(600 \mathrm{~V})$ to the specimen. This technique achieves annealing temperatures of $1000^{\circ} \mathrm{C}$ within a few minutes. The heating rate and resulting surface temperature depend on the emission current that can be tuned in the range of $8-25 \mathrm{~mA}$ by changing the current applied on the filament.

The applied annealing temperatures $T_{\mathrm{c}}$ are given in Table 3.1. Prior to any scattering experiment, the surfaces were cleaned by one cleaning cycle of Ar-ion sputtering and annealing.

Table 3.1: Overview of applied surface annealing temperature $\left({ }^{\circ} \mathrm{C}\right)$.

\begin{tabular}{lcc} 
Sample & $T_{\mathrm{c}}$ & $T_{\mathrm{f}}$ \\
\hline \hline $\mathrm{Ni}$ & 600 & 720 \\
$\mathrm{Cu}$ & 600 & - \\
$\mathrm{Pd}$ & 650 & 900 \\
$\mathrm{Ag}$ & 600 & - \\
$\mathrm{Pt}$ & 700 & 700 \\
$\mathrm{Au}$ & 700 & - \\
$\alpha-\mathrm{Al}_{2} \mathrm{O}_{3}$ & 600 & 600 \\
$50-1 \mathrm{~nm} \mathrm{AlOx}$ & 500 & 500 \\
$\mathrm{ML} \mathrm{AlOx}$ & 700 & 700 \\
Graphene & 300 & -
\end{tabular}

The investigated samples of $\mathrm{Ni}, \mathrm{Pd}$ and $\mathrm{Pt}$ were found to be very sensitive to contamination. Carbon or hydrogen impurities could be already detected after measuring for $30-90$ min. These samples could be cleaned during the measurements by annealing rapidly (flushing $2-5 \mathrm{~min}$ ) at an appropriate annealing temperature $T_{\mathrm{f}}$ (Table 3.1). It has to be noted that this procedure only works sufficiently for $2-3$ times. Thus, the surface had to be cleaned again by a full cleaning cycle of Ar-ion sputtering and annealing typically after $3-4 \mathrm{~h}$ of measurements.

In contrast to the metal surfaces, the insulating and ultra-thin layered samples could not be cleaned by Ar-ion sputtering. The aluminum oxide surfaces could instead be cleaned in an oxygen environment (partial pressure $\left.10^{-6} \mathrm{O}_{2}\right)$ at $T_{\mathrm{c}}$ for several hours $(>20 \mathrm{~h}$ ). As a daily procedure, the aluminum oxide samples were annealed for $30 \mathrm{~min}$ in such an oxygen environment.

The in situ generated graphene layered Ni surfaces daily were cleaned by annealing for 5 min in UHV at $T_{\mathrm{c}}$, which is sufficient to guarantee a well-defined surface. Possible adsorbed hydrogen atoms on the graphene layer desorb below $300^{\circ} \mathrm{C}$ surface temperature.116 


\subsubsection{Atomic oxygen covered $\mathrm{Pt}(111)$ surface}

Oxygen covered $\mathrm{Pt}(111)$ surface was prepared in situ by dosing a clean single crystal $\mathrm{Pt}(111)$ surface for $5 \mathrm{~min}$ with oxygen $\left(10^{-6} \mathrm{mbar}\right)$ at room temperature. This procedure results in a saturated coverage of $0.25 \mathrm{ML}$ atomic oxygen with a preferential $p(2 \times 2)$ orientation. 113 The surface structure was determined by LEED. The resulting LEED pattern are shown and discussed in Section 3.6 .2 in Fig. 3.12

\subsubsection{ALD prepared films}

ALD was performed in a hot and cold wall, cross laminar flow, multiple substrate reactor with attached mass spectrometer (MS) and quartz crystal microbalance (QCM). 117 Trimethylaluminum (TMA) and degassed demineralized water were used in order to produce thin films of aluminum oxide. Both precursors were evaporated at room temperature and injected separately into the reactor with pulse times of $150 \mathrm{~ms}$. Argon served as a heated carrier and purging gas (purge time: $2 \mathrm{~min}$ ). In the reactor the precursors reacted on heated $\mathrm{SiO}_{2}$ or $\mathrm{Pt} / \mathrm{SiO}_{2}$ surfaces $\left(75^{\circ} \mathrm{C}\right)$. The experimental conditions lead to a aluminum oxide growth rate of $1.94 \pm 0.06 \AA$ /cycle (corresponding to $0.85 \pm 0.03 \mathrm{ML} /$ cycle). Polycrystalline platinum layers were synthesized using $\mathrm{MeCpPtMe}_{3}$ and oxygen. Gaseous $\mathrm{MeCpPtMe}_{3}\left(\right.$ at $70^{\circ} \mathrm{C}$ ) reacted on silicon at $325^{\circ} \mathrm{C}$. Pulse times of $5 \mathrm{~s} \mathrm{MeCpPtMe}_{3}$ and $5 \mathrm{~ms} \mathrm{O}_{2}$, separated by Ar flushing, result in polycrystalline films of platinum with preferential (111) orientation and growth rates of $0.7 \pm 0.1 \AA$ /cycle (corresponding to $0.25 \pm 0.04 \mathrm{ML} /$ cycle). Fig. 3.13 illustrates the prepared aluminum oxide surfaces.

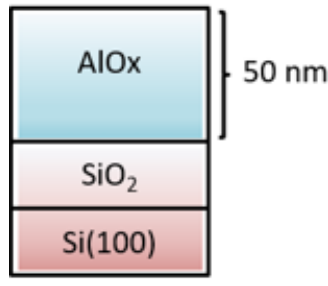

I

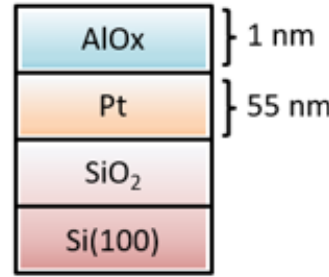

II

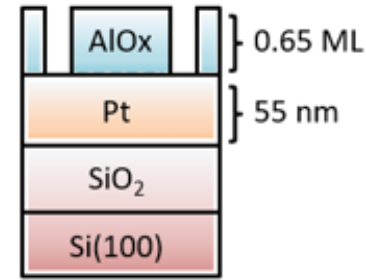

III

Figure 3.13: Illustration of ALD prepared and investigated aluminum oxide surfaces. 
Surfaces of fully closed aluminum oxide layers with $50 \mathrm{~nm}$ (I) and $1 \mathrm{~nm}$ thickness on $55 \mathrm{~nm}$ platinum (II) as well as a not fully closed ML of aluminum oxide on $55 \mathrm{~nm}$ platinum (III) were synthesized on silicon $\left(\mathrm{Si} / \mathrm{SiO}_{2}\right)$ waver. The approximately $1 \mathrm{~nm}$ thin aluminum oxide layer on platinum (II) was grown by 5 ALD cycles, whereas a not fully closed monolayer of aluminum oxide (III) was formed by a single ALD cycle.

\section{Characterization of ALD prepared films}

The investigated aluminum oxide layers were characterized with respect to their roughness, composition and thickness by C. Volksmann.117

Layer thickness of sample II was investigated by TEM (Transition Electron Microscopy) (Fig. 3.14 a). The aluminum oxide layer exhibits a homogeneous layer thickness of $1.0(3) \mathrm{nm}$ with a $55 \mathrm{~nm}$ platinum sublayer. The $1 \mathrm{~nm}$ layer thickness corresponds to approximately 4-5 monolayers of aluminum oxide. The composition of sample II was studied by Electron Energy Loss Spectroscopy (EELS). EELS mapping of $\mathrm{Al}($ red) and $\mathrm{O}$ (blue) (Fig. 3.14 b,c) revealed a stoichiometry of $\mathrm{Al}_{2} \mathrm{O}_{3.5}$.

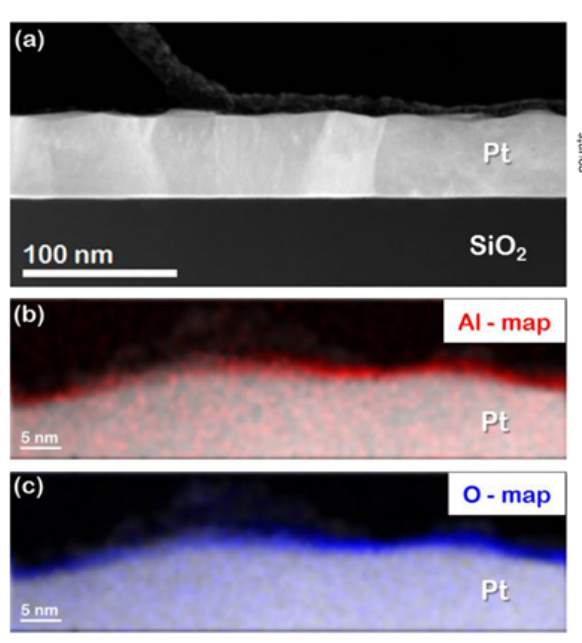

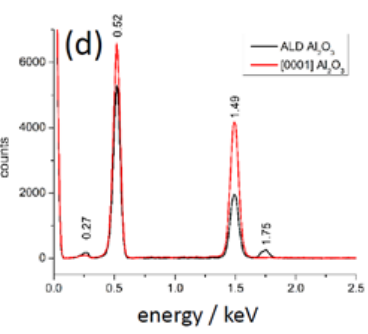
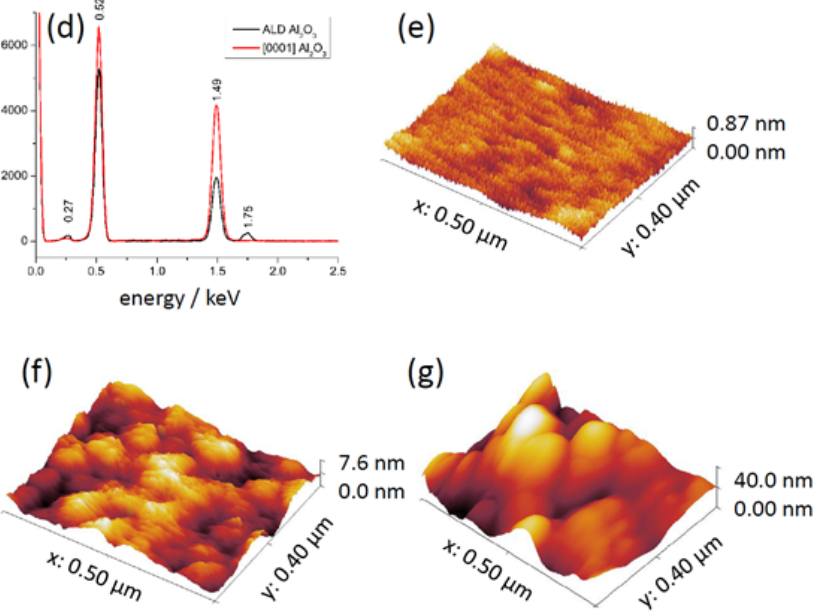

Figure 3.14: (a) TEM image and (b, c) EELS mapping of sample II $\left(1 \mathrm{~nm} \mathrm{AlO}_{\mathrm{x}}\right.$ on $55 \mathrm{~nm} \mathrm{Pt}$ on a $\mathrm{SiO}_{2}$ substrate). (d) EDX spectrum of sample I (50 nm $\mathrm{AlO}_{\mathrm{x}}$ on $\mathrm{SiO}_{2}$ substrate, black) compared to $\alpha-\mathrm{Al}_{2} \mathrm{O}_{3}$ (red) and AFM 3D image of (e) $\alpha-\mathrm{Al}_{2} \mathrm{O}_{3}(0001)$, (f) $\mathrm{AlO}_{\mathrm{x}}(1 \mathrm{~nm}) / \mathrm{Pt}$ and $(\mathrm{g}) \mathrm{AlO}_{\mathrm{x}}(0.65 \mathrm{ML}) / \mathrm{Pt}$. Reprinted with permission from 117. Copyright 2018 American Chemical Society.

EDX (Energy Dispersive X-ray spectroscopy) (Fig. 3.14 d) was performed on sample I (black curve) in order to examine the composition of the $50 \mathrm{~nm}$ thick aluminum oxide layer. The $\mathrm{O}$ signal $(0.52 \mathrm{eV})$ was affected by the underlaying $\mathrm{SiO}_{2}$ substrate $(\mathrm{Si}$ signal at 
$1.75 \mathrm{eV}$ ) and had therefore to be corrected. A single crystalline $\alpha-\mathrm{Al}_{2} \mathrm{O}_{3}$ sample (Sample IV) was measured as a reference (red curve). The investigations exhibit a stoichiometry of sample I of $\mathrm{Al}_{2} \mathrm{O}_{3.7}$.

The surface roughness of the investigated aluminum oxide samples was determined by AFM (Atomic Force Microscopy). Fig. 3.14 (e-g) presents representative AFM images of the single crystalline $\alpha-\mathrm{Al}_{2} \mathrm{O}_{3}$ surface (e), the $1 \mathrm{~nm} \mathrm{AlO}_{\mathrm{x}}$ on $55 \mathrm{~nm}$ platinum surface (f) and the $0.65 \mathrm{ML} \mathrm{AlO}_{\mathrm{x}}$ on $55 \mathrm{~nm}$ platinum surface (g). The single crystalline surface exhibits a smooth surface with a maximum roughness of $1 \mathrm{~nm}$ and a mean roughness $R_{a}$ of $0.10 \mathrm{~nm}$. The roughness of the ALD prepared aluminum oxide surfaces is affected by the substrate. The $1 \mathrm{~nm} \mathrm{AlO}_{\mathrm{x}}$ on $55 \mathrm{~nm}$ platinum revealed a maximum roughness of $7.9 \mathrm{~nm}$ and a $R_{a}$-value of $1.54 \mathrm{~nm}$. The $0.65 \mathrm{ML} \mathrm{AlO}_{\mathrm{x}}$ on $55 \mathrm{~nm}$ platinum revealed a maximum roughness of $22.4 \mathrm{~nm}$ and a $R_{a}$-value of $4.51 \mathrm{~nm}$. The $R_{a}$-values of all investigated samples are given in Table 3.2 .

The synthesized surfaces initially contained approximately $2 \%$ carbon contamination. After insertion into the UHV scattering machine and bake-out, the films were cleaned as described in Section 3.7. AES was subsequently utilized to confirm the surface cleanliness and to estimate elementary surface composition of the ALD prepared aluminum oxide films. Auger spectra of $\alpha-\mathrm{Al}_{2} \mathrm{O}_{3}, \mathrm{ALD}$ prepared $1 \mathrm{~nm}$ aluminum oxide $\left(\mathrm{AlO}_{\mathrm{x}}\right)$ on $55 \mathrm{~nm}$ platinum, ALD prepared not fully closed ML of $\mathrm{AlO}_{\mathrm{x}}$ on $55 \mathrm{~nm} \mathrm{Pt}$ and oxygen covered Pt(111) are depicted in Fig. 3.15 .

The Auger signals are assigned to the corresponding elements. The Al LMM signal and the Pt Auger signals at $E_{\text {kin }}<100 \mathrm{eV}$ overlap and thus cannot be used for Al:O surface stoichiometry determination according to Equation 3.13. Therefore, the less intense $\mathrm{Al} \mathrm{KLL} \mathrm{signal}\left(E_{\mathrm{kin}} \approx 1350 \mathrm{eV}\right.$ ) was used with a worse signal to noise ratio. The $\mathrm{AlO}_{\mathrm{x}}(1 \mathrm{~nm}) / \mathrm{Pt}$ Auger spectrum is used as a reference to estimate the Al:O surface ratio of the other investigated aluminum oxide surfaces. The elemental composition of the $\mathrm{AlO}_{\mathrm{x}}(1 \mathrm{~nm}) / \mathrm{Pt}$ sample was estimated by EELS. Furthermore, the aluminum oxide coverage of the not fully closed $\mathrm{ML} \mathrm{AlO}_{\mathrm{x}}$ sample can be estimated by using the oxygen covered $\mathrm{Pt}(111)$ surface (coverage 25\%) as a reference. According to the AES measurements, the not fully closed monolayer of $\mathrm{AlO}_{\mathrm{x}}$ on $55 \mathrm{~nm}$ Pt exhibits an oxygen coverage of $65 \pm 10 \%$. The estimated coverage is slightly lower than the expected coverage based on the experimentally determined growth rate $(0.85 \mathrm{ML} /$ cycle $)$.

For the sake of simplicity, from this point onwards the sample is described as 0.65 ML $\mathrm{AlO}_{\mathrm{x}}$ on Pt. Further it should be noted that sample IV contains a non negligible chemical vapor deposition (CVD) component of the Pt phase due to a high oxygen background pressure in the reactor during the preparation process. 


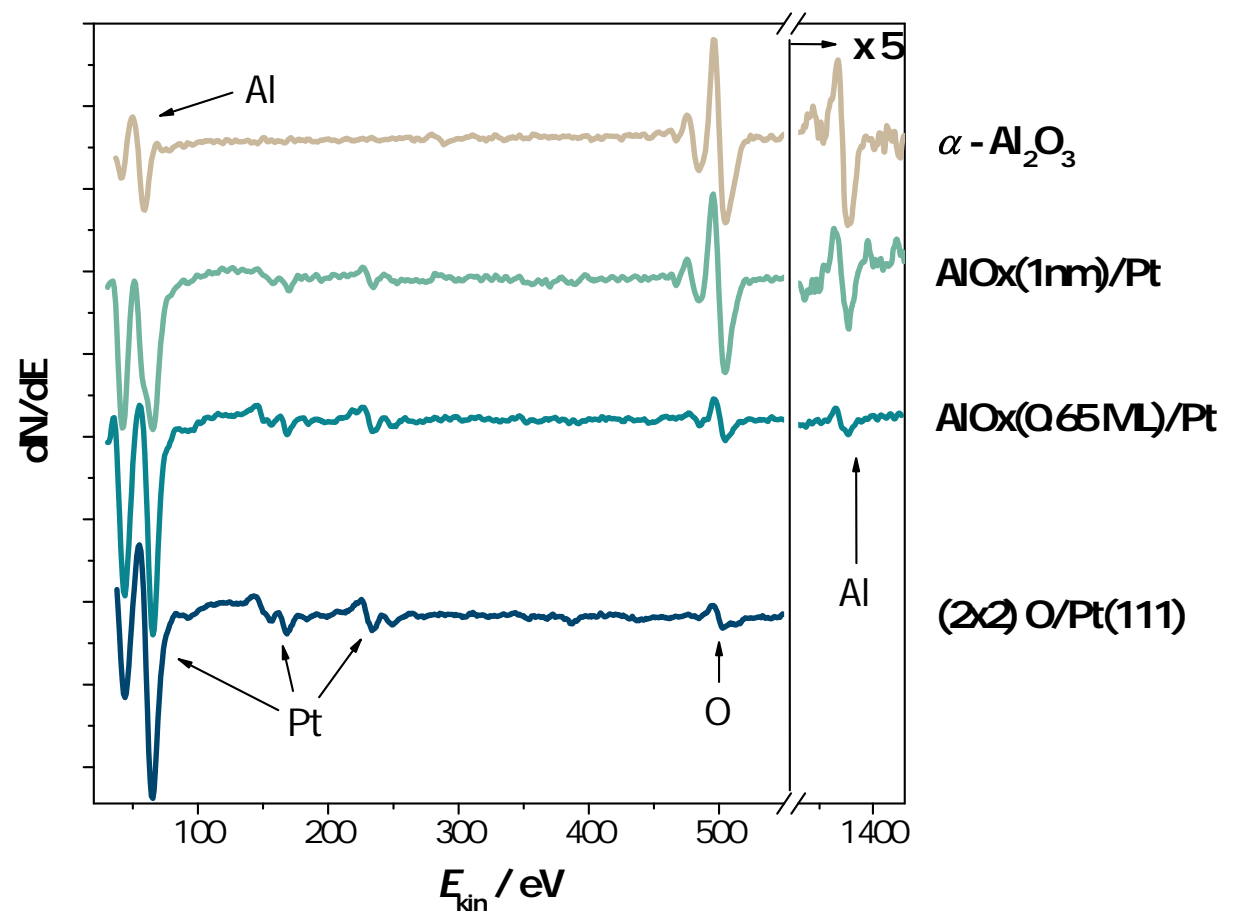

Figure 3.15: Auger spectra of investigated oxide films. The Al KLL signal $\left(E_{\text {kin }} \approx 1350 \mathrm{eV}\right)$ was magnified (x5). The Al LMM signal and the Pt signals $\left(E_{\text {kin }}<100 \mathrm{eV}\right)$ of the $\mathrm{AlO}_{\mathrm{x}}$ thin films overlap. Therefore, information of the Al:O stoichiometry has been derived from the Al KLL signal. Reprinted with permission from [117]. Copyright 2018 American Chemical Society.

Discrepancies between EELS and AES measurements are observed for the $50 \mathrm{~nm}$ sample (II). According to AES the investigated $50 \mathrm{~nm}$ sample even exhibits a higher $\mathrm{Al} / \mathrm{O}$ ratio than the single crystal surface (I) which might indicate a strong fluctuation of the $\mathrm{Al} / \mathrm{O}$ ratio within the $50 \mathrm{~nm}$ thick aluminum oxide layer. An overview of the characterized surfaces can be found in Table 3.2

Table 3.2: Estimated interface and film stoichiometry and surface roughness of the ALD prepared $\mathrm{AlO}_{\mathrm{x}}$ films and $\alpha-\mathrm{Al}_{2} \mathrm{O}_{3}$ sample.

\begin{tabular}{lcccc} 
Sample & $\begin{array}{c}\mathrm{Al}: \mathrm{O} \text { ratio } \\
(\text { EELS \& EDX })\end{array}$ & $\begin{array}{c}\mathrm{Al} \text { :O ratio } \\
(\text { AES })\end{array}$ & $\begin{array}{c}\text { Roughness } \\
/ \mathrm{nm}\end{array}$ & $\begin{array}{c}\text { Maximum } \\
\text { Roughness } / \mathrm{nm}\end{array}$ \\
\hline \hline I: $\alpha-\mathrm{Al}_{2} \mathrm{O}_{3}$ & $\mathrm{Al}_{2} \mathrm{O}_{3}$ & $\mathrm{Al}_{2} \mathrm{O}_{2.3}$ & 0.10 & 0.87 \\
II: $\mathrm{AlO}_{\mathrm{x}}(50 \mathrm{~nm})$ & $\mathrm{Al}_{2} \mathrm{O}_{3.7}$ & $\mathrm{Al}_{2} \mathrm{O}_{1.9}$ & 0.66 & n.d. \\
III: $\mathrm{AlO}_{\mathrm{x}}(1 \mathrm{~nm}) / \mathrm{Pt}$ & $\mathrm{Al}_{2} \mathrm{O}_{3.5}$ & $\mathrm{Al}_{2} \mathrm{O}_{3.5}$ & 1.54 & 7.6 \\
IV: $\mathrm{AlO}_{\mathrm{x}}(0.65 \mathrm{ML}) / \mathrm{Pt}$ & - & $\mathrm{Al}_{2} \mathrm{O}_{>3.5}$ & 4.51 & 22.4
\end{tabular}


AES measurements indicate that the aluminum oxide surfaces provide a lower $\mathrm{Al} / \mathrm{O}$ ratio than the bulk material. The surface roughness increases with decreasing aluminum oxide film thickness. The Al:O ratio is consistently lower for the ALD prepared surfaces compared to single crystalline aluminum oxide. This observation is in accordance with previous results whereupon ALD produces aluminum oxide films with lower atomic density and lower $\mathrm{Al} / \mathrm{O}$ ratio.118 119 The single crystalline $\mathrm{Pt}(111)$ surface was not investigated for its surface roughness. Nevertheless, it is believed that the surface roughness is much lower than $1 \mathrm{~nm}$ due to several cleaning cycles.

\subsubsection{Graphene on $\mathrm{Ni}(111)$}

In this thesis two different types of monolayer graphene on $\mathrm{Ni}(111)$ (A: rot. $\mathrm{Gr} / \mathrm{Ni}(111)$ and $\mathrm{B}:(1 \mathrm{x} 1) \mathrm{Gr} / \mathrm{Ni}(111))$ were synthesized in situ via CVD using two different conditions on a clean single crystal $\mathrm{Ni}(111)$ surface (principle reaction scheme see Fig. 3.16):

A: Rotated graphene on $\mathrm{Ni}(111)$ is formed upon surface exposure of ethylen $\left(\mathrm{C}_{2} \mathrm{H}_{4}\right.$, $p=1.4 \cdot 10^{-6}$ mbar, dosing time: $3 \mathrm{~h}$ ) at $T_{\mathrm{s}}=550^{\circ} \mathrm{C}$ on a clean $\mathrm{Ni}(111)$ surface. Afterwards, the ethylen is removed from the chamber and the sample is kept for approximately 5 min at $T_{\mathrm{s}}=550^{\circ} \mathrm{C}$ under UHV before the surface is cooled down.

B: $(1 \times 1)$ graphene on $\mathrm{Ni}(111)$ is formed by a two-step process: First a clean $\mathrm{Ni}(111)$ sample is hold at $T_{\mathrm{s}}=400^{\circ} \mathrm{C}$ and exposed to ethylen $\left(p=1.2 \cdot 10^{-6} \mathrm{mbar}\right)$ for approximately $1 \mathrm{~h}$. This forms a single layer $\mathrm{Ni}_{2} \mathrm{C}$-phase. Subsequent heating of the surface at surface temperatures between 450 and $500^{\circ} \mathrm{C}$ under continuous ethylen exposure for approximately $2 \mathrm{~h}$ forms a single $(1 \times 1)$ oriented graphene on $\mathrm{Ni}(111)$. Afterwards, alike rotated graphene the sample is kept for another $5 \mathrm{~min}$ at $T_{\mathrm{s}}=500^{\circ} \mathrm{C}$ under UHV before surface cooling in order to remove remaining ethylen gas.

The pristine $\mathrm{Ni}(111)$ substrate was cleaned as described in Sec. 3.7 .

Quality and order of the graphene surfaces was measured by LEED and AES. The LEED images in Fig. 3.16 clearly demonstrate that with procedure A rotated graphene is formed. The arched like LEED pattern result from rotated graphene, the bright spots with sixfold symmetry result from the $\mathrm{Ni}(111)$ surface.

It has to be noted, that with procedure $\mathrm{B}$ an almost perfect $(1 \times 1)$ oriented graphene layer on $\mathrm{Ni}(111)$ can be formed (LEED only shows one type of pattern with sixfold 


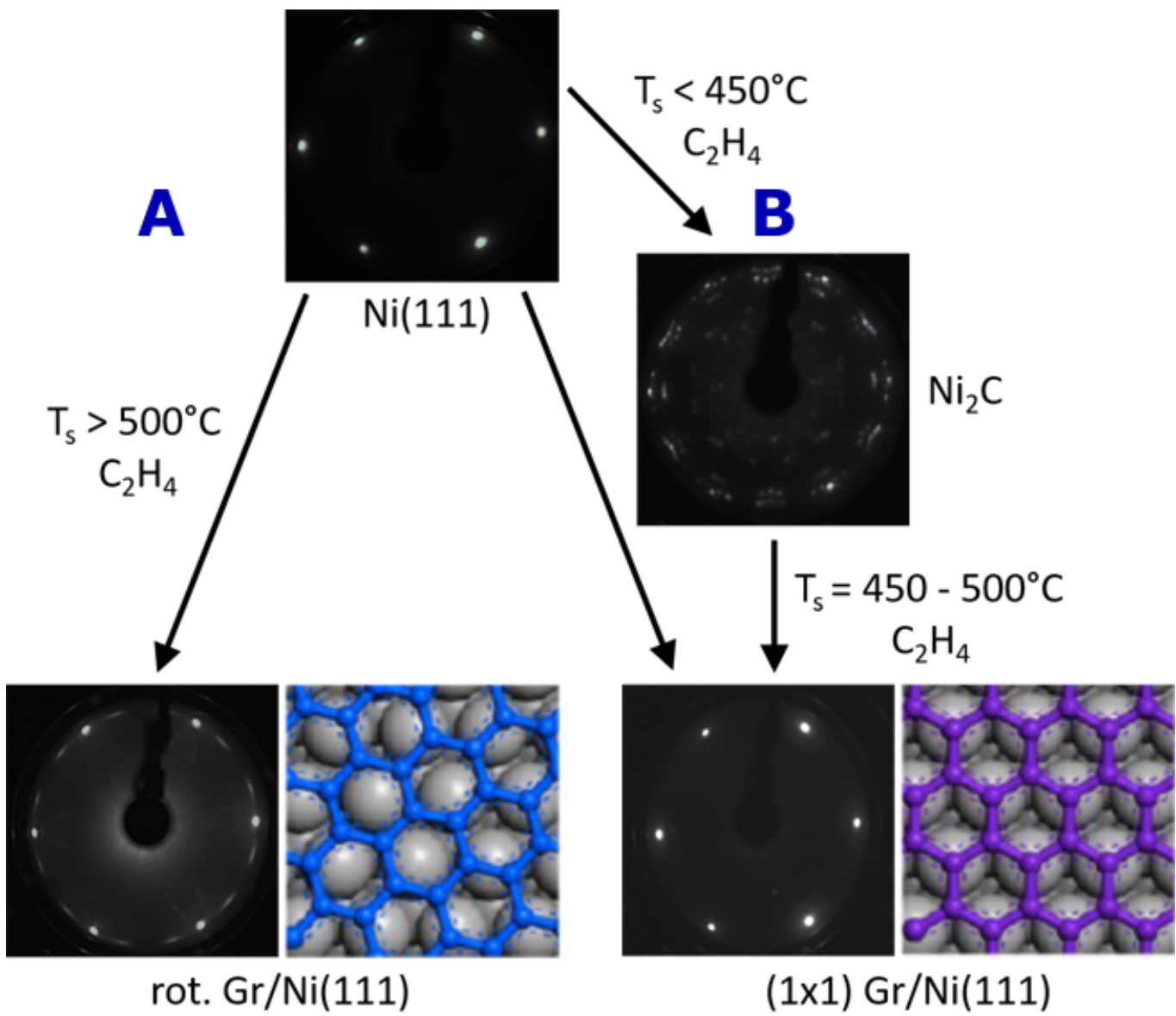

Figure 3.16: Synthesis pathway for the production of rotated graphene (left pathway) and (1 x 1$)$ oriented graphene (right pathway) on pristine $\mathrm{Ni}(111)$, respectively. The surface phase was measured by LEED. According to literature, the dominant surface configuration of rot. graphene and (1 x 1) graphene on $\mathrm{Ni}(111)$ is illustrated, respectively. Reprinted with permission from [89]. Copyright 2013 American Chemical Society. 
symmetry), while in reaction procedure A the formation of rotated graphene competes with the formation of perfectly aligned graphene. Thus, the surface consists of domains of rotated and $(1 \times 1)$ oriented graphene. Nevertheless, the brightness of the arched pattern are indicative for a high abundance of rotated graphene domains. Next to the LEED image a possible rotated graphene configuration on $\mathrm{Ni}(111)$ is illustrated.

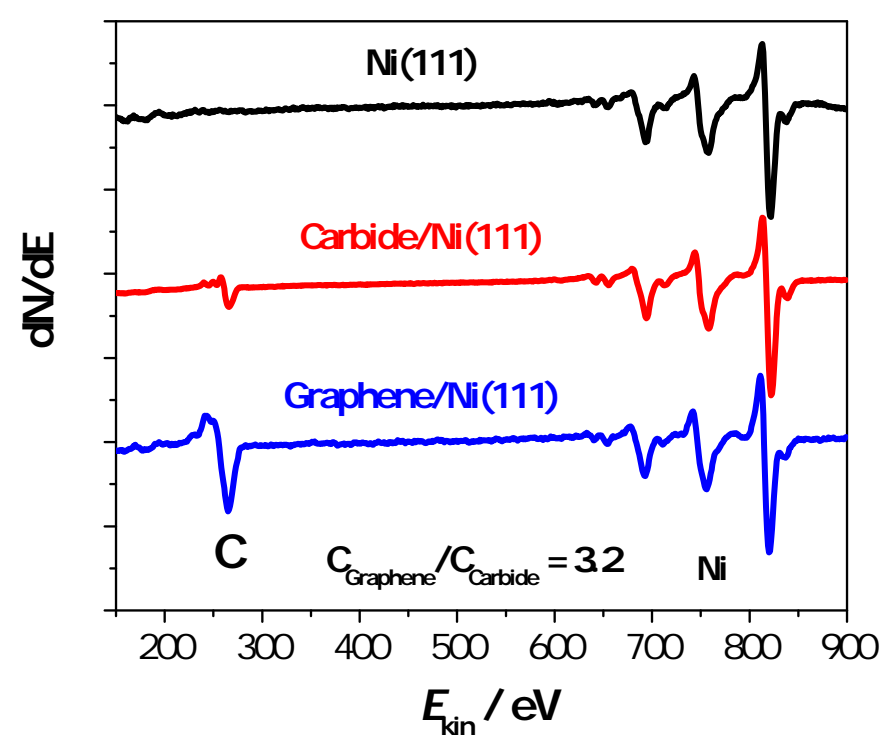

Figure 3.17: Auger spectrum of $\mathrm{Ni}(111)$ (black), Carbide on $\mathrm{Ni}(111)$ (blue) and (1 x 1) graphene (red) on Ni(111) presenting the carbon KVV and nickel LMM, LMV and LVV Auger peaks (ordered: low $G \rightarrow$ high $E_{\text {kin }}$ ). The Auger spectra were recorded with a primary electron beam of $3 \mathrm{eV}$.

AES was used to ensure the cleanliness of the surface and to meaure the progress of the graphene growth process. Fig. 3.17 depicts the Auger spectrum of the pristine $\mathrm{Ni}(111)$ (black), carbide on $\mathrm{Ni}(111)$ (red) and (1 x 1) graphene (blue) on $\mathrm{Ni}(111)$ surfaces. Only Auger peaks of nickel and carbon can be observed in the spectra which underlines the cleanliness of the surfaces. The graphene and carbide layers show an additional peak at $272 \mathrm{eV}$ resulting from the carbon atoms of the graphene/carbide layer. The graphene layer shows a approximately three times higher carbon Auger peak than the carbide surface consistent with values from literature. $\frac{88}{}$ The end of the graphene growth process was determined by the saturation of the carbon Auger peak. An Auger spectrum of rotated graphene on $\mathrm{Ni}(111)$ is not shown, as it is basically identical to $(1 \times 1)$ graphene on $\mathrm{Ni}(111)$ Auger spectrum with similar carbon to nickel Auger peak ratios. 


\section{$4 \mathrm{H}$ and $\mathrm{D}$ atom scattering from transition metal surfaces}

In this chapter the results from inelastic $\mathrm{H}$ and $\mathrm{D}$ atom scattering experiments from six $f c c$ transition metals ( $\mathrm{Au}, \mathrm{Pt}, \mathrm{Ag}, \mathrm{Pd}, \mathrm{Cu}$ and $\mathrm{Ni}$ ) with a (111) surface cut are discussed. The atomic and electronic structure of these metal surfaces has been previously outlined in Section 2.2, while their characterization and cleaning procedures were described in Section 3.7

First, comparative experimental studies and molecular dynamics simulations of hydrogen atom scattering from $\mathrm{Au}(111)$ and insulating solid multilayers of xenon answered the question of how hydrogen atoms can effectively transfer their inherent translational energy to equilibrate with the solid surface. 28

Second, hydrogen and deuterium scattering experiments were performed, which gave rise to a very low but significant isotope effect that could be theoretically explained. 52 Previous studies showed, that small molecule - surface interactions can be strongly influenced by the electronic structure and work function of the investigated metal surfaces. $120|121| 45|46| 122 \mid 123$

Thus, in the last comparative study various $f c c$ transition metal surfaces were studied in order to elucidate whether, and if so how the nature of the metals influences the atom solid interaction during the adsorption process. 


\section{1 $\mathrm{H}$ atoms scattering from $\mathrm{Au}(111)$ and insulating xenon surfaces}

$\mathrm{H}$ atom scattering experiments were performed on a single crystal $\mathrm{Au}(111)$ surface at room temperture and a bulk like xenon layer on $\mathrm{Au}(111)$ at $45 \mathrm{~K}$ with an incidence energy of $E_{\mathrm{i}, \mathrm{H}}=2.76 \mathrm{eV}$ and an incidence and scattering angle of $\vartheta_{\mathrm{i}}=\vartheta_{\mathrm{s}}=45^{\circ}$ a The obtained translational energy loss distributions (the scattered $\mathrm{H}$ atom flux with respect to the energy loss of the incidence $\mathrm{H}$ atom beam) from $\mathrm{Au}(111)$ and insulating xenon layer are compared in the left panel of Fig. 4.1 (Au: red dots; Xe: blue dots). The presented spectra are normalized to the total $\mathrm{H}$ atom flux.

The black solid line shows the measured translational energy distribution of the incidence $\mathrm{H}$ atom beam, which differs drastically for $\mathrm{Au}(111)$ and Xe. Scattering from xenon exhibits a narrow energy distribution with a most probable energy loss of $E_{\text {loss }}=46 \mathrm{meV}$, which is lower than the expected energy loss limit derived from a simple Baule collision model (Baule limit: $83 \mathrm{meV}$, indicated as vertical cyan arrow). The Baule limit predicts the overall maximum energy loss, thus, the prediction might always overestimate the observed energy loss at a certain incidence and scattering angle (here $\vartheta_{\mathrm{i}}=\vartheta_{\mathrm{s}}=45^{\circ}$ ). Additionally, deviations may also result from hydrogen atoms experiencing a higher effective mass of the xenon surface atoms during the collision process.

In contrast, $\mathrm{Au}(111)$ exhibits a much broader distribution with a 20 times higher average energy loss $\left(E_{\text {loss }}=910 \mathrm{meV}\right)$ than obtained for xenon. This observation cannot be explained within a simple collision model (Baule limit: $56 \mathrm{meV}$, shown as vertical cyan arrow in the right panel) indicating that electronic excitation is involved in collision processes between $\mathrm{H}$ atoms and gold.

A theoretical model (see Section 2.1.4) treating mechanical energy transfer to Au lattice motion as well as electronic excitation of a Au metal surface was developed by Janke et al.51 to give further insight into the energy transfer mechanism and a detailed view of the energy, angle and isotope dependencies.

In Fig. 4.1, right panel, the experimentally obtained energy loss distribution spectrum from $\mathrm{Au}(111)$ (red dots) is compared to energy distributions resulting from MD simulations including (blue line) and neglecting (black line) electronic excitation.

The inset shows translational energy distribution spectra of $\mathrm{H}$ atoms scattered from $\mathrm{Au}(111)$ (black, red and blue dots) measured at three different incidence energies (in-

\footnotetext{
${ }^{\text {a Xenon layer was deposited on a clean } \mathrm{Au}} \mathrm{u}(111)$ surface hold at $45 \mathrm{~K}$ by dosing with xenon gas for 300 Langmuir, producing a sufficiently thick xenon layer to ensure that the underneath Au surface does not influence the scattering process on xenon. 124
} 
Exp.: Au(111) vs. Xe

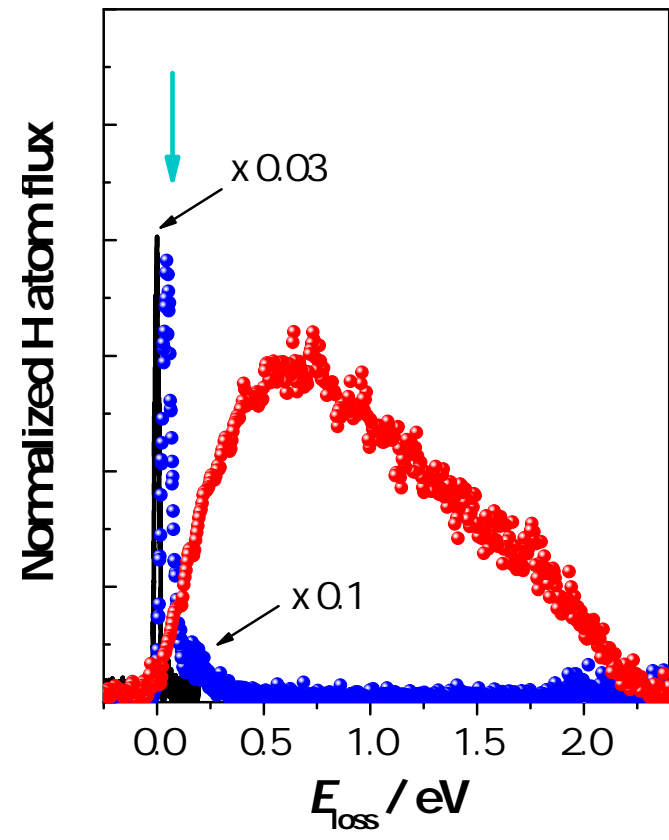

Au(111): Exp. vs. Sim

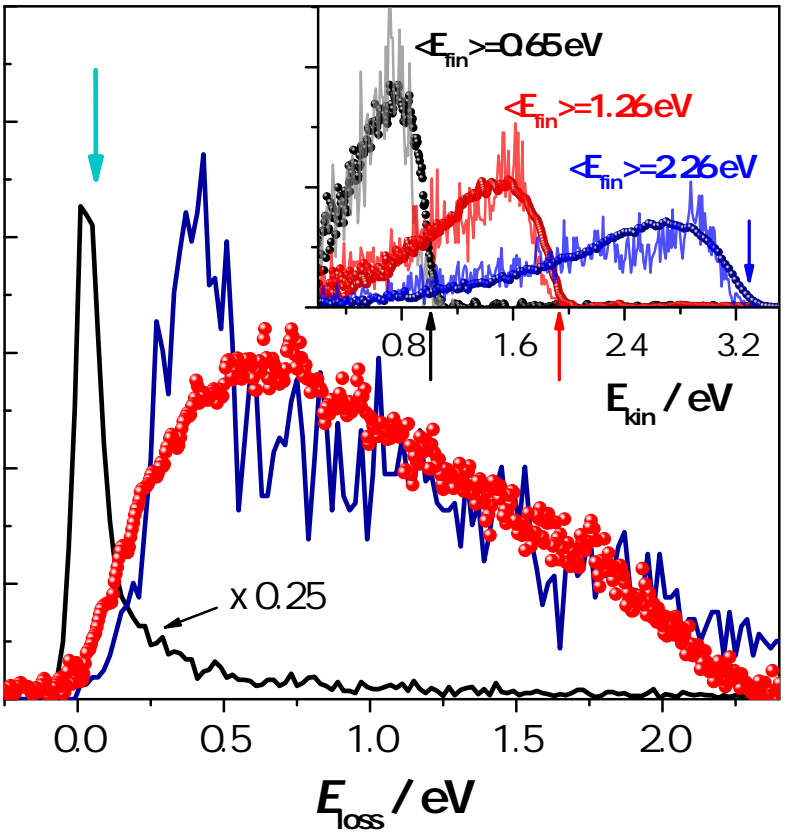

Figure 4.1: Left panel: Translational energy loss distribution spectra of $\mathrm{H}$ atoms scattered from $\mathrm{Au}(111)$ (red dots) and solid xenon (blue dots) at $E_{\mathrm{i}}=2.76 \mathrm{eV}$. The energy distribution of the incident $\mathrm{H}$ atom beam is shown as black line. The vertical arrow indicates the energy loss of an $\mathrm{H}$ atom colliding with a xenon atom derived from a simple collision model. Right panel: Comparison of energy loss distribution from $\mathrm{Au}(111)$ experimentally obtained (red dots) and theoretical simulations including (blue line) and neglecting (black line) electronic excitation, respectively. The vertical arrow indicates the energy loss for a simple hard cube collision between an $\mathrm{H}$ and an $\mathrm{Au}$ atom. The inset shows comparisons of ED spectra from $\mathrm{Au}(111)$ obtained from experiment (dots) and theory (pale lines) at three different incidence energies (marked as vertical arrows): $E_{\mathrm{i}}=0.99 \mathrm{eV}$ (black, grey), $E_{\mathrm{i}}=1.92 \mathrm{eV}$ (red, pale red) and $E_{\mathrm{i}}=3.33 \mathrm{eV}$ (blue, pale blue). The average kinetic energies of the scattered $\mathrm{H}$ atoms are indicated as $\left\langle E_{\mathrm{fin}}\right\rangle$. The experimental conditions are $\vartheta_{\mathrm{i}}=\vartheta_{\mathrm{i}}=45^{\circ}$

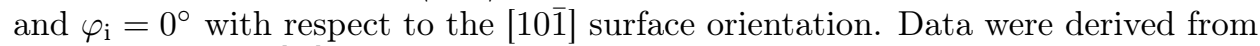
Bünermann et al.28 
cidence energies marked as vertical arrows). Theoretically derived energy distributions including electronic excitation (grey, pale red and pale blue lines) (EMT-LDFA-MD simulations) are in good agreement with experimental results for all studied incidence kinetic energies.

All energy distributions are broad and structureless. H atoms scattered from $\mathrm{Au}(111)$ lose on average a constant fraction of their incidence energy $\left\langle E_{\text {loss }}\right\rangle=0.28 \pm 0.01 \cdot E_{\mathrm{i}}$ independent of the investigated initial kinetic energy of the incidence $\mathrm{H}$ atom beam.

The theoretical model including electronic excitation simulates the experimentally derived data notably well. In contrast, MD simulations neglecting electronic excitation cannot describe the experimentally observed energy distribution which shows that hydrogen atom adsorption is not a result from multiple electronically adiabatic collisions caused by a conversion of normal to parallel $\mathrm{H}$ atom momentum as previously presumed in various studies. $\frac{125}{125}$ Further, these observations confirm the hypothesis that electronic excitation of the solid is the most dominant energy dissipation channel for $\mathrm{H}$ atoms colliding with a $\mathrm{Au}(111)$ surface at all investigated incidence energies and determines the adsorption mechanism, predominantly a penetration-resurfacing mechanism. According to theory, the most efficient electronic excitation is at subsurface binding sites while the strongest $\mathrm{H}-\mathrm{Metal}$ bonds are formed on the surface. Thus, $\mathrm{H}$ atom impinging and sticking on the surface initially populate favorably at subsurface sites where they lose their translational energy before back-migration to the surface and subsequent adsorption. 


\subsection{Dependence on experimental conditions}

In order to gain deeper understanding of $\mathrm{H}$ atom scattering processes on $\mathrm{Au}(111)$, the final translational energy and angular distributions of the scattered hydrogen atoms in dependence of the scattering angle were measured at various incidence energies and angles at $300 \mathrm{~K}$ surface temperature (see e.g. Fig. 4.2).
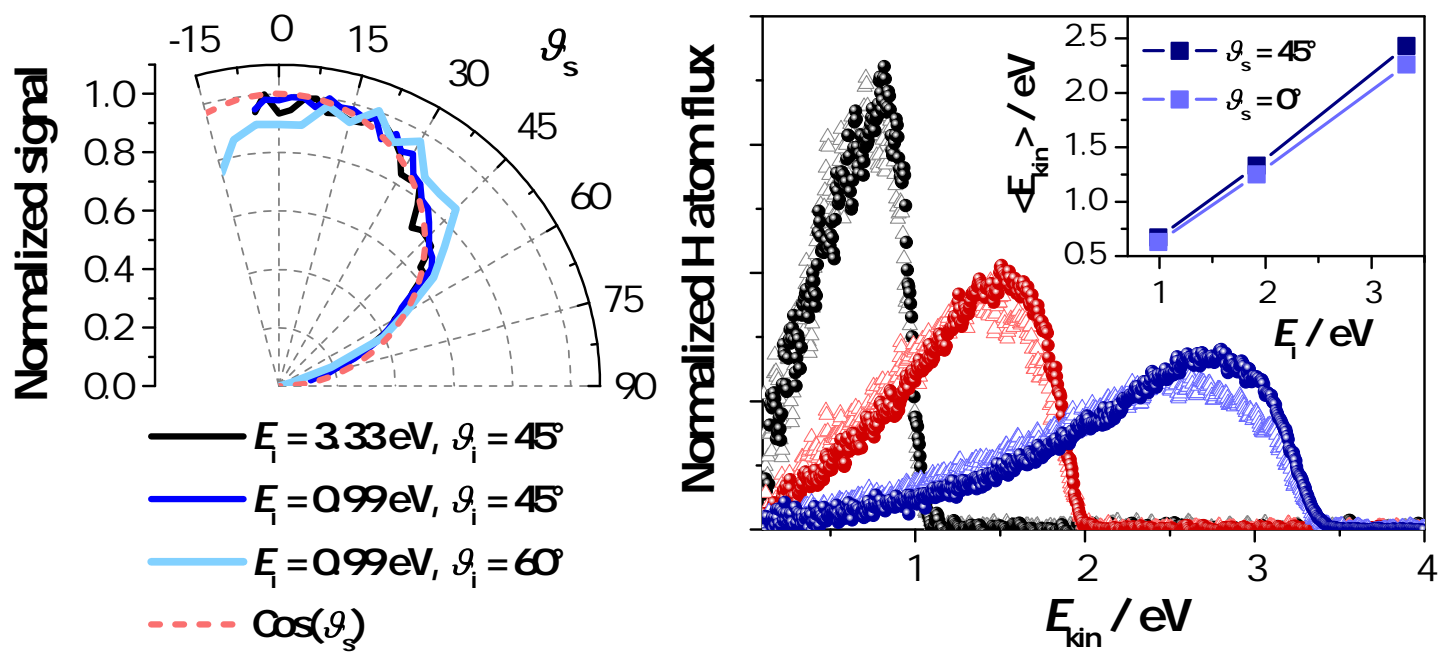

Figure 4.2: Comparison of angular distribution and energy distribution spectra of $\mathrm{H}$ atoms scattered from $\mathrm{Au}(111)$ under various experimental conditions. Left panel: Angular distribution recorded at various incident energies and angles. Black and dark blue line: $\vartheta_{\mathrm{i}}=45^{\circ}$ and $E_{\mathrm{i}}=3.33$ and $0.99 \mathrm{eV}$, respectively. The angular distributions follow almost a cos distribution (red dashed line). No differences are observed for angular distributions resulting from different incident energies. Small changes occur when changing the incidence angle: $\vartheta_{\mathrm{i}}=60^{\circ}$ and $E_{\mathrm{i}}=0.99 \mathrm{eV}$ (pale blue line). Right panel: Energy distribution measured at different incidence energies: $E_{\mathrm{i}}=0.99$ (black, grey), 1.92 (red) and $3.33 \mathrm{eV}$ (blue), and two different scattering angles: $\vartheta_{\mathrm{s}}=45^{\circ}$ (dark blue, dark red and black circles) and $\vartheta_{\mathrm{s}}=0^{\circ}$ (pale blue, pale red and grey dots triangles). The incidence angle was fixed at $\vartheta_{\mathrm{i}}=45^{\circ}$. The inset shows the weighted arithmetic average kinetic energy of the scattered $\mathrm{H}$ atoms with respect to the incidence energy for two different scattering angles: $\vartheta_{\mathrm{s}}=45^{\circ}$ (dark blue) and $\vartheta_{\mathrm{s}}=0^{\circ}$ (pale blue). The azimuth angle was fixed at $\varphi_{\mathrm{i}}=0^{\circ}$.

Angular distribution spectra represent the total $\mathrm{H}$ atom signal with respect to the scattering angle for one incidence energy and a specific incidence angle. Here, angular distributions (left panel) are shown at an incidence angel of $\vartheta_{\mathrm{i}}=45^{\circ}$ and an incidence energy of $E_{\mathrm{i}}=3.33$ (black line) as well as $0.99 \mathrm{eV}$ (dark blue line). In order to study the incidence angle dependence, a measured angular distribution with $\vartheta_{\mathrm{i}}=60^{\circ}$ at $E_{\mathrm{i}}=0.99 \mathrm{eV}$ (pale blue line) is also presented.

The angular distributions are broad and follow a cosine distribution fit (red dashed line) 
and no differences are observed with respect to the incidence energies. However, with increasing incidence angle, the angular distribution slightly shifts towards higher angles. In the right panel, energy distributions measured at different incident energies are displayed: $E_{\mathrm{i}}=0.99$ (black), 1.92 (red) and $3.33 \mathrm{eV}$ (blue), and two different scattering angles $\vartheta_{\mathrm{s}}=45^{\circ}$ (dark blue, dark red and black) and $\vartheta_{\mathrm{s}}=0^{\circ}$ (pale blue, pale red and grey dots). The incidence angle was fixed at $\vartheta_{\mathrm{i}}=45^{\circ}$. For all investigated experimental conditions, scattered $\mathrm{H}$ atoms exhibit a rather broad energy loss distribution. The energy distribution for lower scattering angles is shifted significantly towards higher energy losses.

The inset shows the determined average kinetic energy $\left\langle E_{\text {kin }}\right\rangle$ of the scattered $\mathrm{H}$ atoms with respect to the incidence energy for the two investigated scattering angles. $\mathrm{H}$ atoms lose a constant fraction of their incidence energy with respect to their scattering angle:

Table 4.1: Average energy loss of $\mathrm{H}$ atoms scattering from $\mathrm{Au}(111)$ at two different scattering angles.

\begin{tabular}{cc}
$\vartheta_{\mathrm{s}}$ & $\left\langle E_{\mathrm{loss}}\right\rangle / E_{\mathrm{i}}$ \\
\hline \hline $45^{\circ}$ & $0.28 \pm 0.01$ \\
$0^{\circ}$ & $0.33 \pm 0.01$
\end{tabular}

The azimuth angle was fixed at $\varphi_{\mathrm{i}}=0^{\circ}$ with respect to the $[10 \overline{1}]$ surface direction. Additional studies revealed no influence changing the azimuthal angle.

The angular distribution and the average energy loss of the scattered $\mathrm{H}$ atoms are not affected by the incidence energy. The observed cosine shape of the angular distributions can indicate a trapping-desorption mechanism, which would suggest that $\mathrm{H}$ atoms reside sufficiently long enough on the surface to equilibrate thermally with the surface and lose all information about their incidence conditions which would subsequently lead to a Maxwell-Boltzmann energy distribution of the scattered $\mathrm{H}$ atoms. However, a small dependence on the incidence and scattering angle are obtained and the observed energy distributions are not thermal, thus, trapping-desorption cannot be the predominant scattering mechanism.

As has been outlined in the previous section, this broad energy distribution can be explained by electron hole pair excitation of the metal and modeled by non-adiabatic EMT-LFDA-MD simulations. A simulated energy distribution for $E_{\mathrm{i}}=3.33 \mathrm{eV}$ and $\vartheta_{\mathrm{i}}=45^{\circ}$ is shown in Fig. 4.3 (dark blue shaded area).

Analysis of the simulated MD trajectories indicate that the probability distribution of the scattered $\mathrm{H}$ atoms can be subdivided into various scattering mechanisms: single bounce (cyan), double bounce (green) and more than two bounce collisions and/or subsurface collisions (beige) between an $\mathrm{H}$ atom and $\mathrm{Au}$ atoms. 


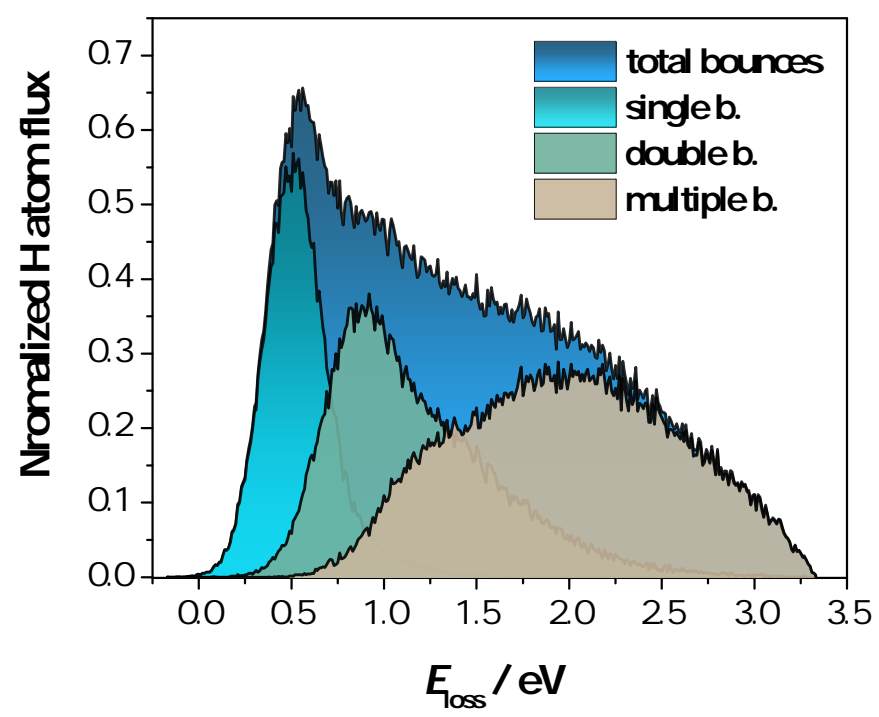

Figure 4.3: Analysis of the overall energy distribution of $\mathrm{H}$ atoms scattering from $\mathrm{Au}(111)$ obtained from MD simulation including electron-hole-pair excitation (dark blue shaded area). The energy probability distribution can be subdivided into three classes of trajectories: single bounce (cyan shaded area), double bounce (green shaded area), and more than two bounce collisions (beige shaded area) between an $\mathrm{H}$ atom and $\mathrm{Au}$ atoms. Incidence conditions are $E_{\mathrm{i}}=3.33 \mathrm{eV}$ and $\vartheta_{\mathrm{i}}=45^{\circ} .126$

Single bounce collision can be assigned to a direct inelastic scattering mechanism. H atoms which scatter directly from the surface retain their incidence angle information. This leads to angular and energy distributions which are strongly affected by the applied experimental conditions.

At the other extreme, $\mathrm{H}$ atoms, which experience multiple bounce collisions and/or go subsurface, lose their incidence angle information and scatter in all possible directions and thus show no dominant preference angle yielding broad angular and energy distributions independent of the incidence and scattering angle as well as higher energy losses.

According to MD-simulations for incidence energies of $E_{\mathrm{i}, \mathrm{H}}=0.99 \mathrm{eV}, 1.92 \mathrm{eV}$ and $3.33 \mathrm{eV}$, $\mathrm{H}$ atoms impinging on the metal surface and scatter back undergo on average 1.6, 2.2 and 2.8 collisions, respectively. 127 In conclusion, the energy distributions of hydrogen atoms scattered from $\mathrm{Au}(111)$ exhibit only small dependence on the incidence and scattering angle. The combination of various mechanism can explain the observed results. Similar observations were obtained for other transition metal surfaces (see Chapter 4.4) as well. After various experimental conditions were investigated, in the following only specified conditions will be discussed. 


\subsection{Isotope effect in scattering experiment from $\mathrm{Au}(111)$ and $\operatorname{Ag}(111)$ surfaces}

The isotope effect of hydrogen and deuterium atoms scattered from $\mathrm{Au}(111)$ and $\mathrm{Ag}(111)$ was studied and compared to EMT-LDFA-MD calculations. .52

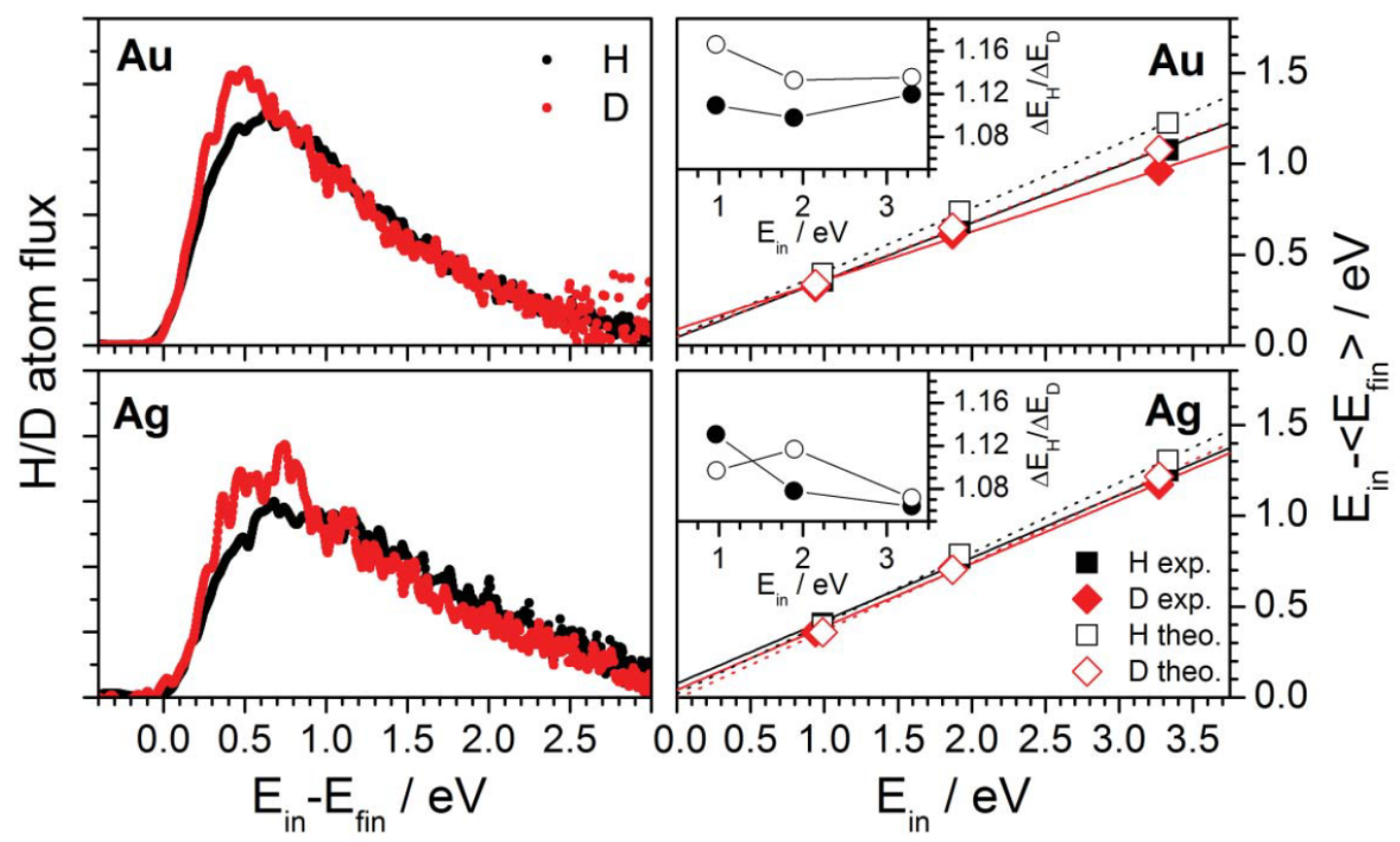

Figure 4.4: Left panels: Representative energy loss distribution spectra of $\mathrm{H}$ (black) and D (red) atoms scattered from $\mathrm{Au}(111)$ (upper panel) and $\mathrm{Ag}(111)$ (lower panel) at $E_{\mathrm{i}, \mathrm{H}}=3.33 \mathrm{eV}, E_{\mathrm{i}, \mathrm{D}}=3.27 \mathrm{eV}, \vartheta_{\mathrm{i}}=\vartheta_{\mathrm{s}}=45^{\circ}$ and $\varphi_{\mathrm{i}}=0^{\circ}$. Right panels: Determined average energy loss of $\mathrm{H}$ (black) and D (red) atoms scattered from $\mathrm{Au}(111)$ and $\operatorname{Ag}(111)$ with respect to incidence kinetic energy, $E_{\text {in }}$. Experimental results (filled symbols) are compared to results of MD simulations (open symbols). Insets: Isotopic ratios, $\Delta E_{\mathrm{H}} / \Delta E_{\mathrm{D}}$, plotted against the incidence energy. 52

As can be seen in Fig. 4.4, depicting energy loss distributions of H (black) and D (red) atoms scattered from $\mathrm{Au}(111)$ and $\mathrm{Ag}(111)$ show no significant differences.

The right panels show the determined average energy loss (experiment: filled symbols, theory: open symbols) of $\mathrm{H}$ and $\mathrm{D}$ atoms with respect to incidence kinetic energy $E_{\text {in }}$. The inset plots the experimentally observed isotopic ratios, $\Delta E_{\mathrm{H}} / \Delta E_{\mathrm{D}}$, against the incidence energy.

Classically treated inelastic scattering process predict a strong isotope effect, as the atomic 
mass of the hydrogen isotopes differ s by a factor of two. Comparing the experimental results from $\mathrm{H}$ and $\mathrm{D}$ atom scattering, for both investigated surfaces $\mathrm{H}$ and $\mathrm{D}$ atoms experience a broad energy loss distribution with only marginally differences. Only a small isotope effect of approximately $10 \%$ is observed according to which D atoms consistently show a less effective energy transfer to the surface $\left\langle E_{\text {loss }, \mathrm{H}}\right\rangle /\left\langle E_{\text {loss }, \mathrm{D}}\right\rangle \approx 1.1$.

This observations are contradictory to classical calculations. Thus, subsequently theoretical MD calculations were performed to elucidate these observations.

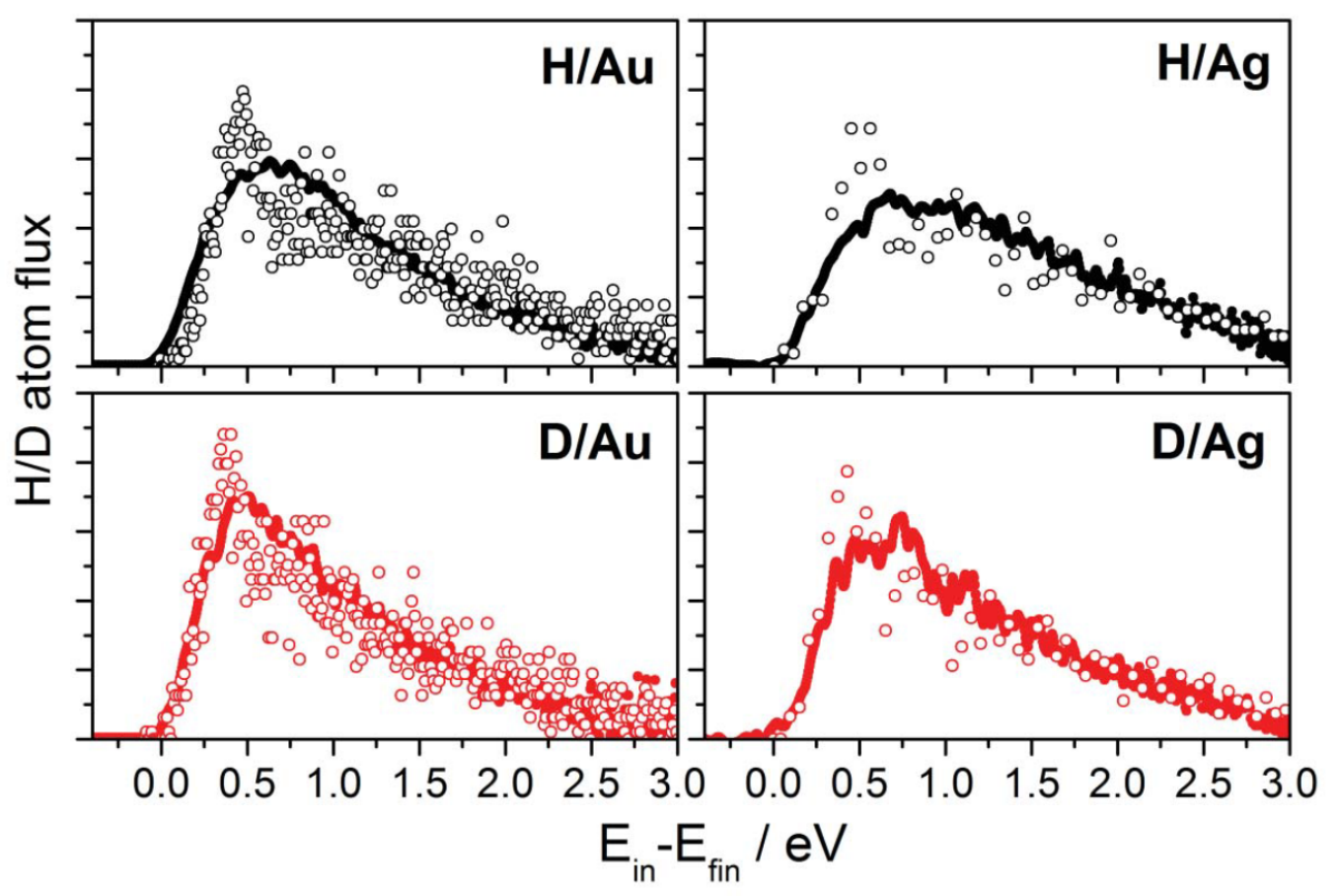

Figure 4.5: Comparison of full translational energy loss distributions of $\mathrm{H}$ (black) and D (red) atoms scattered from $\mathrm{Au}(111)$ (left panels) and $\mathrm{Ag}(111)$ (right panel) obtained from experiment (solid lines) and MD simulations (open circles) at $E_{\mathrm{i}, \mathrm{H}}=3.33 \mathrm{eV}$, $E_{\mathrm{i}, \mathrm{D}}=3.27 \mathrm{eV}, \vartheta_{\mathrm{i}}=\vartheta_{\mathrm{s}}=45^{\circ}$ and $\varphi_{\mathrm{i}}=0^{\circ} .52$

Representative experimental (solid lines) and EMT-LFDA-MD simulated (open circles) translational energy loss distributions of $\mathrm{H}$ and $\mathrm{D}$ atoms scattered from $\mathrm{Au}(111)$ and $\operatorname{Ag}(111)$ are compared in Fig 4.5. The calculated and experimentally obtained energy distribution spectra and the derived average energy losses are in good agreement.

According to the MD simulations, the low isotope effect on the investigated transition metals results from a compensation of adiabatic phonon excitation and non-adiabatic electronic excitation. 128

This can be explained as follow: On one hand, for mechanical excitation (treated adiabatic), 
the higher mass of the $\mathrm{D}$ atoms induces an enhanced phonon excitation probability compared to $\mathrm{H}$ atoms.

$$
\frac{\Delta E_{\mathrm{ad}}(D)}{\Delta E_{\mathrm{ad}}(H)} \approx \frac{m_{\mathrm{D}}}{m_{\mathrm{H}}}=2
$$

On the other hand, the efficiency probability for electronic excitation (non-adiabatic) is significantly higher for $\mathrm{H}$ atoms, as for a specified incidence energy, the velocity for $\mathrm{H}$ atoms is always higher than for D atoms by a factor of $\sqrt{2}$.

$$
\frac{\Delta E_{\mathrm{na}}(D)}{\Delta E_{\mathrm{na}}(H)} \approx \frac{v_{\mathrm{D}}}{v_{\mathrm{H}}} \approx \sqrt{\frac{m_{\mathrm{H}}}{m_{\mathrm{D}}}}=\sqrt{\frac{1}{2}}
$$

The MD simulations further predict an unequal contribution of these both competing effects. For mechanical excitation contribution percentage: $10 \%(\mathrm{H})$ and $21 \%(\mathrm{D})$; for electronic excitation contribution percentage: $90 \%(\mathrm{H})$ and $79 \%(\mathrm{D})$.

In conclusion, the observed weak isotope effect is explained by two competing process (phonon and electronic excitation) and their unequal contribution for both isotopes. 


\subsection{Scattering dynamics on six $f c c$ transition metal surfaces}

In the previous sections a methodology for investigating $\mathrm{H}$ and $\mathrm{D}$ atoms scattering processes could be established using $\mathrm{Au}(111)$ and $\mathrm{Ag}(111)$ surfaces. In the following, the same methodology was extended to four additional $f c c$ transition metals ( $\mathrm{Pt}, \mathrm{Pd}, \mathrm{Cu}, \mathrm{Ni}$ ) in a combined experimental and theoretical study, which has been also published. 127

\subsubsection{Experimental results}

The angular distributions and energy distributions at different incidence and scattering angle in the range of $\vartheta_{\mathrm{i}}=30-60^{\circ}$ and $\vartheta_{\mathrm{s}}=0-60^{\circ}$ were measured for $\mathrm{H}$ and $\mathrm{D}$ atoms scattered from different transition metal surfaces at surface temperatures of $300 \mathrm{~K}$.

First of all, as previously found for $\mathrm{Au}(111)$ surfaces, no or only little dependence of the angular and energy distribution spectra was observed for all transition metal surfaces, when changing the incidence energy or incidence and scattering angle conditions. Thus, only selected spectra are shown here.

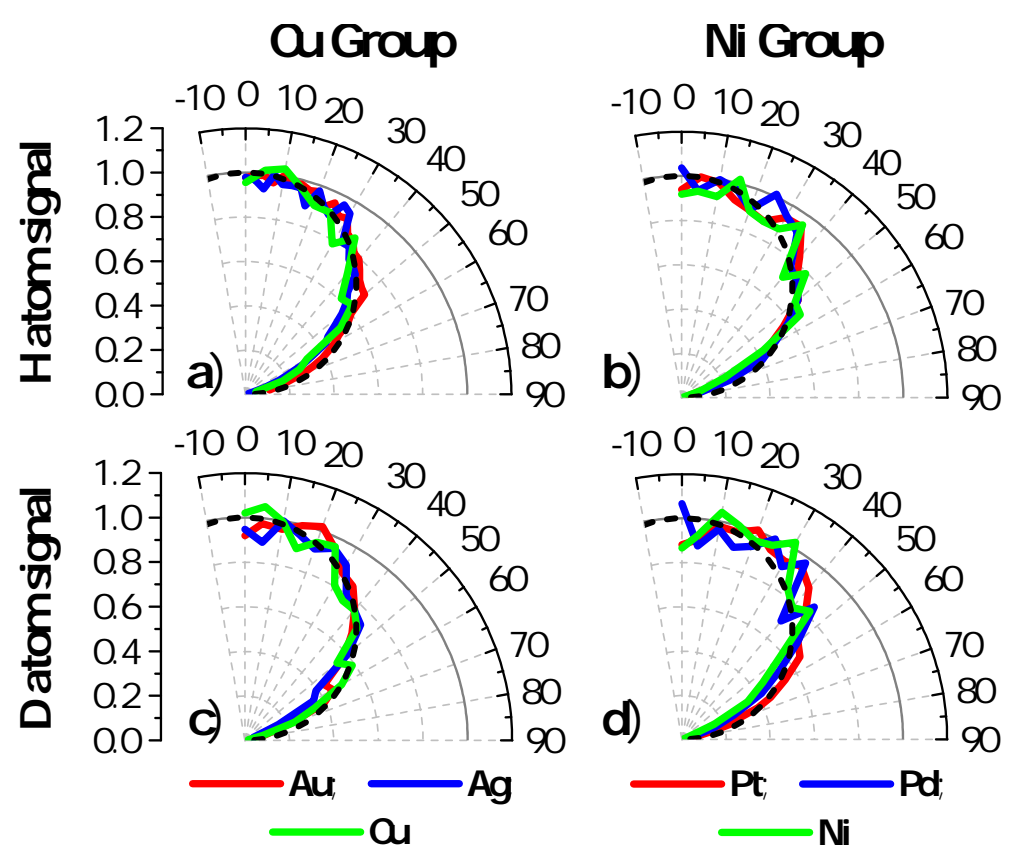

Figure 4.6: Experimentally determined angular distribution spectra of $\mathrm{H} / \mathrm{D}$ atoms (upper/lower panels) scattered from six various $f c c d$-metal surfaces ( $\mathrm{Cu}$ group: left; Ni group: right). Experimental conditions: $E_{\mathrm{i}, \mathrm{H}}=1.92 \mathrm{eV}, E_{\mathrm{i}, \mathrm{D}}=1.87 \mathrm{eV}, \vartheta_{\mathrm{i}}=45^{\circ}$. All angular distributions are normalized to the average peak maximum.127 Dashed black line: Cosine fit. 
As can be seen in Fig. 4.6 (exp. cond.: $E_{\mathrm{i}, \mathrm{H}}=1.92 \mathrm{eV}, E_{\mathrm{i}, \mathrm{D}}=1.87 \mathrm{eV}, \vartheta_{\mathrm{i}}=45^{\circ}$ ), for all investigated transition metals ( $\mathrm{Cu}$ group: left; $\mathrm{Ni}$ group: right) similar broad and structureless angular distributions are obtained for both isotopes (H: upper panels, D: lower panels), which can be fitted by a broad cosine fit (dashed black line).

The experimentally measured energy probability distributions of H/D (left/right panel) atoms reveal a significant atomic mass dependent trend (Fig. 4.7). Selected energy distributions are shown at $E_{\mathrm{i}, \mathrm{H}}=1.92 \mathrm{eV}, E_{\mathrm{i}, \mathrm{D}}=1.87 \mathrm{eV}, \vartheta_{\mathrm{i}}=\vartheta_{\mathrm{s}}=45^{\circ}$ (spectra are ordered according to atomic mass $\mathrm{Au}-\mathrm{Ni}$ : top - bottom).

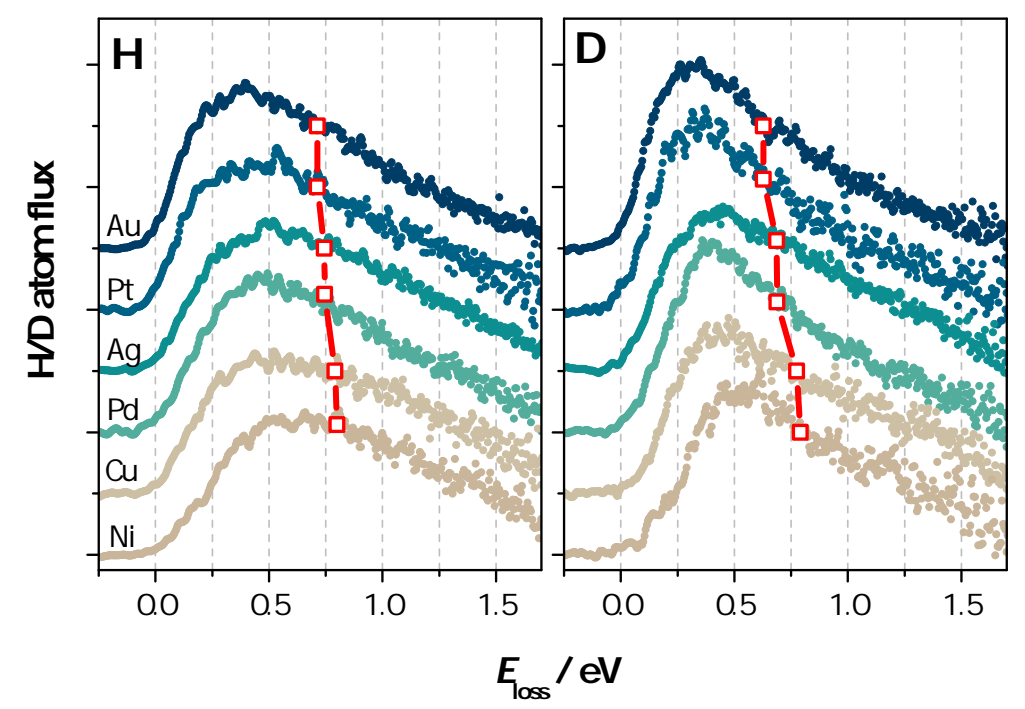

Figure 4.7: Experimentally determined energy loss distributions of H/D atoms (left/right panel) scattered from $f c c d$-metal surfaces. Experimental conditions: $E_{\mathrm{i}, \mathrm{H}}=1.92 \mathrm{eV}$, $E_{\mathrm{i}, \mathrm{D}}=1.87 \mathrm{eV}, \vartheta_{\mathrm{i}}=\vartheta_{\mathrm{s}}=45^{\circ}$. Unfilled red squares correspond to determined average energy losses. 127

The translational energy distributions of all investigated metals exhibit a very similar shape. The width changes marginally leading to small changes in the overall energy loss. Specifically at high scattering energies (close to zero energy loss) the scattering intensity decreases for lower mass metals leading in general to an increased energy loss.

The determined weighted average energy loss (unfilled red squares Fig. 4.7) may best illustrate the changes in the overall energy loss. From Au to Ni (ordered according to mass) the average energy loss increases for both isotopes $\left\langle E_{\text {loss }, \mathrm{H}}\right\rangle=0.71 \mathrm{eV}-0.80 \mathrm{eV}$; $\left\langle E_{\text {loss, }, \mathrm{D}}\right\rangle=0.63 \mathrm{eV}-0.79 \mathrm{eV}$ (for details see Table 4.2). 
Table 4.2: Determined weighted arithmetic final mean energy loss of $\mathrm{H} / \mathrm{D}$ atoms scattering from six metals at $E_{\mathrm{i}, \mathrm{H}}=1.92 \mathrm{eV}, E_{\mathrm{i}, \mathrm{D}}=1.87 \mathrm{eV}, \vartheta_{\mathrm{i}}=\vartheta_{\mathrm{s}}=45^{\circ}$. For comparison atomic mass and work function $\Phi$ are also listed.

\begin{tabular}{lcccc} 
Metal & Atomic mass $/ \mathrm{au}$ & $\Phi / \mathrm{eV}$ & $\left\langle E_{\text {loss }, \mathrm{H}}\right\rangle / \mathrm{eV}$ & $\left\langle E_{\text {loss }, \mathrm{D}}\right\rangle / \mathrm{eV}$ \\
\hline \hline $\mathrm{Au}(111)$ & 197.0 & 5.31 & 0.713 & 0.628 \\
$\mathrm{Pt}(111)$ & 195.1 & 5.93 & 0.713 & 0.628 \\
$\mathrm{Ag}(111)$ & 107.9 & 4.74 & 0.744 & 0.687 \\
$\mathrm{Pd}(111)$ & 106.4 & 5.6 & 0.745 & 0.689 \\
$\mathrm{Cu}(111)$ & 63.5 & 4.94 & 0.791 & 0.774 \\
$\mathrm{Ni}(111)$ & 58.7 & 5.35 & 0.801 & 0.791
\end{tabular}

The energy loss at an incidence energy of approximately $2 \mathrm{eV}$ seemingly correlates to the atomic mass for both isotopes but appears to be unaffected by electronic configuration and work function. Next, energy distribution (see Fig. 4.10) and aforementioned determined average energy loss will be compared for neighboring elements: $\mathrm{Au} \leftrightarrow \mathrm{Pt}, \mathrm{Ag} \leftrightarrow \mathrm{Pd}$, $\mathrm{Cu} \leftrightarrow \mathrm{Ni}$. When comparing the elemental surfaces with unequal DOS at the Fermi level, work function, or atomic structure (reconstruction of $\mathrm{Au}(111)$ ), no significant trends are observed. Similar results of diamagnetic $\mathrm{Cu}$ and ferromagnetic Ni are observed, thus, magnetic influences can be neglected for the scattering processes. However, for surfaces with similar density of states (DOS) but different atomic masses (comparing $\mathrm{Au}$ and $\mathrm{Cu}$ ) $\mathrm{H} / \mathrm{D}$ energy distributions with overall higher energy losses (especially at high scattering energies) for metals with the lighter mass (here $\mathrm{Cu}$ ) are observed. Additionally, the shape of the energy distribution changes significantly for the neighboring $3 d$ metal surfaces compared to their higher homologous pairs.

The weighted average energy loss of the six investigated metal surfaces was further studied in the energy range of approximately $1-3 \mathrm{eV}$ for both isotopes in order to ensure the consistency of the experiment and to corroborate the previously made assumptions. The experimentally determined absolute average energy loss (left panel) with respect to incidence energy and metal (marked in legend) are shown for both isotopes (H: upper panels, D: lower panels) in Figure 4.8 .

Within the investigated energy range, a linear correlation between absolute energy loss and incidence energy values are observed for all metals. Further, the energy loss is effected by the metal surface, obvious by the change in the slope, and increases for lower atomic masses: $\mathrm{Au} \rightarrow \mathrm{Ni}\left\langle E_{\text {loss }, \mathrm{H}}\right\rangle / E_{\mathrm{i}, \mathrm{H}}=0.326 \rightarrow 0.420\left(\mathrm{D}, \mathrm{Au} \rightarrow \mathrm{Ni}:\left\langle E_{\text {loss }, \mathrm{D}}\right\rangle / E_{\mathrm{i}, \mathrm{D}}=0.328 \rightarrow 0.417\right)$. In conclusion it is assumed that the percentage fraction of the $\mathrm{H} / \mathrm{D}$ incidence energy transferred during the surface collision process remains constant for all investigated 
Experiment

Simulation

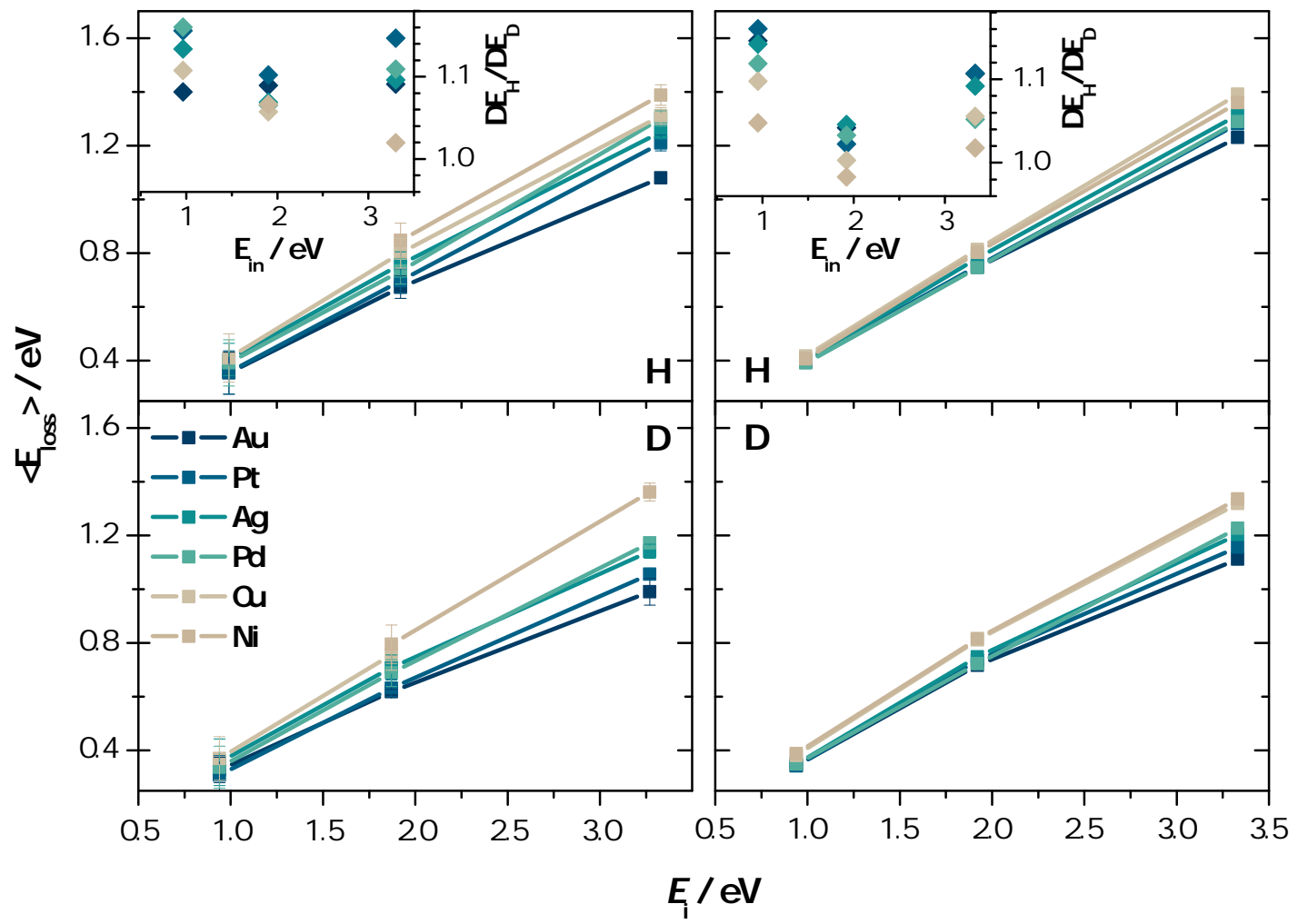

Figure 4.8: Experimental results (left panels) and simulations (right panels) of final average kinetic energy losses (symbols) derived from H/D atom scattering (upper/lower panel) with respect to the incidence kinetic energy and the investigated $f c c d$-metal. Incidence conditions: $\vartheta_{\mathrm{i}}=\vartheta_{\mathrm{s}}=45^{\circ}$. The solid lines are used to guide the eye indicating a linear dependency. Inset: Isotope effect $\left\langle E_{\text {loss, } \mathrm{H}}\right\rangle /\left\langle E_{\mathrm{loss}, \mathrm{D}}\right\rangle \cdot 127$ 
metals. As a result, the percentage average energy loss $\left\langle E_{\text {loss }}\right\rangle / E_{\mathrm{i}}$ is not changing with the incidence energy but is consistently higher for metals with a lower atomic mass. The inset of Fig. 4.8 depicts the isotope effect derived from the average energy loss $\Delta E_{\mathrm{H}} / \Delta E_{\mathrm{D}}$ for the different scattering experiments plotted against the incidence energy. For all metal surfaces, a consistently small isotope effect of approximately $10 \%$ is observed, which is barely affected by the incidence energy. Comparing $3 d, 4 d, 5 d$ metals, the isotope effects becomes more pronounced for the higher homologous.

\subsubsection{Comparison to theoretical calculations}

Based on the observed trends, only an atomic mass effect but no electronic effects would be presumed. In order to varify these assumptions, EMT-LDFA-MD simulations are carried out for all metal surfaces using the previously established methodology for Au(111). 129 To ensure accuracy of the theoretical calculations the experimental results are first compared to the results derived from the simulations.

The right panels of Figure 4.8 present the simulated average kinetic energy losses of $\mathrm{H}$ (top) and D (bottom) for the six $f c c$ metal surfaces. The calculated mean energy losses, isotope effects (see inset) and general trends are in good agreement with the experimentally determined values.

The theoretical results were further compared to the measured angular and energy distributions.

Fig. 4.9 represents angular distributions of $\mathrm{H}$ (top) and D (bottom) of all studied surfaces derived by MD simulations. Each colored line represent a metal surface according to the legend in Fig 4.6. All calculated distributions match the experimentally obtained broad and structureless spectra. The shape can be described as a cosine distribution (dashed black line). However, simulations exhibit a slightly higher scattering probability along the specular angle than experimentally observed.

The measured energy probability distributions can be simulated in good approximation as well. Figure 4.10 clearly demonstrates that the energy distributions determined by the experiment can be calculated by MD simulations for all investigated experimental conditions.

Here, representative energy distributions derived from $\mathrm{H} / \mathrm{D}$ (left/right) atoms scattering from $\mathrm{Pt}(111), \mathrm{Au}(111)$ and $\mathrm{Cu}(111)$ at $E_{\mathrm{i}, \mathrm{H}}=1.92 \mathrm{eV}, E_{\mathrm{i}, \mathrm{D}}=1.87 \mathrm{eV}, \vartheta_{\mathrm{i}}=\vartheta_{\mathrm{s}}=45^{\circ}$ (circles) are compared to MD simulations (lines).

A detailed comparison of the energy distribution spectra reveals that at low energy 
CuGroup

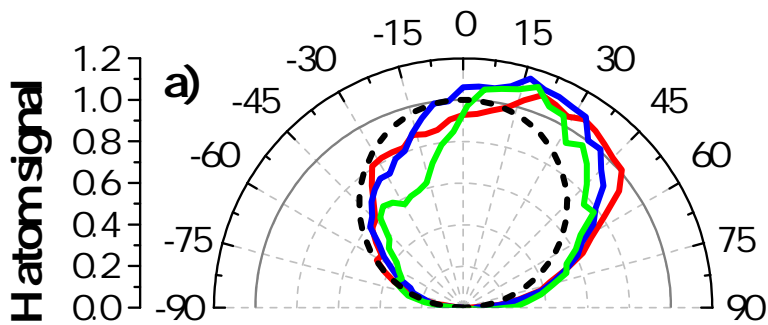

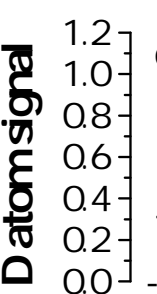

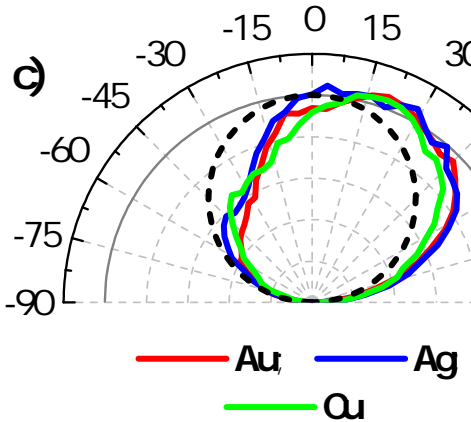

Ni Group
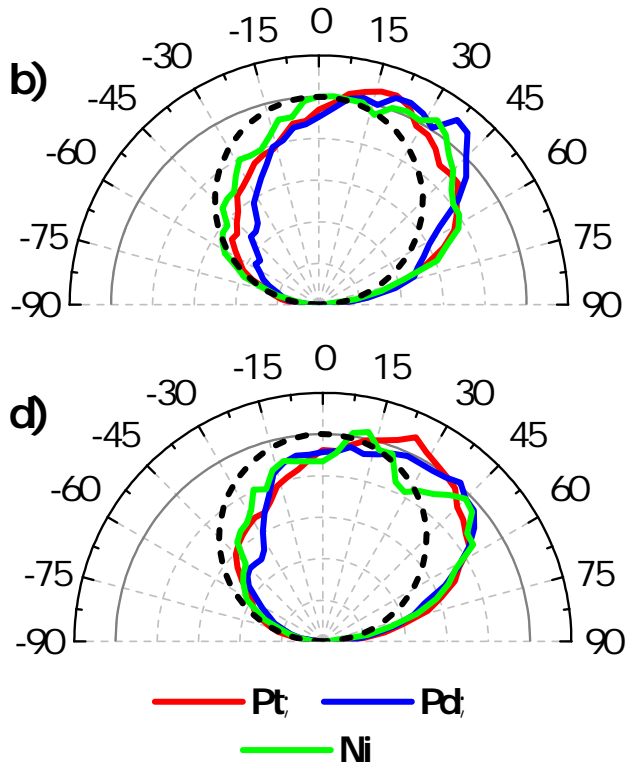

Figure 4.9: Theoretically determined angular distribution spectra of $\mathrm{H} / \mathrm{D}$ atoms (upper/lower panels) scattered from six various $f c c d$-metal surfaces. Same incidence conditions as described in 4.6 . 127

losses the theory predicts a slightly higher scattering intensity. Especially for $\mathrm{H}$ atom scattering, the theory generally overestimated energy losses at high scattering energies (close to zero energy loss). In general, a uniformly better overlap between experiment and theory is obtained for $\mathrm{D}$ atom scattering. The small deviations between theory and experiment might be explained by an overestimation of single collision event probabilities caused by inaccuracies in the determination of the PESs or errors due to the applied classical approximations. However, the comparison demonstrates that the theory is able to reasonably well predict the experimental results of $\mathrm{H}$ and $\mathrm{D}$ atom scattering for all investigated metal surfaces and is therefore suitable for the general description of $\mathrm{H}$ atom scattering processes and explaining the observed trends.

The theory allows to distinguish between two contributing effects: adiabatic mechanical excitation (resulting in phonon excitation) and nonadiabatic electronic excitation (electron-hole-pair excitation), which in sum is able to describe the experimentally observed behavior.

The determined relative average energy loss $\left\langle E_{\text {loss }}\right\rangle / E_{\mathrm{i}}$ of $\mathrm{H}$ (left) and $\mathrm{D}$ (right) is plotted against the atomic mass in Fig. 4.11 (filled red circles) and compared to the calculated average energy loss (filled black squares). Further, the simulated relative proportions of 


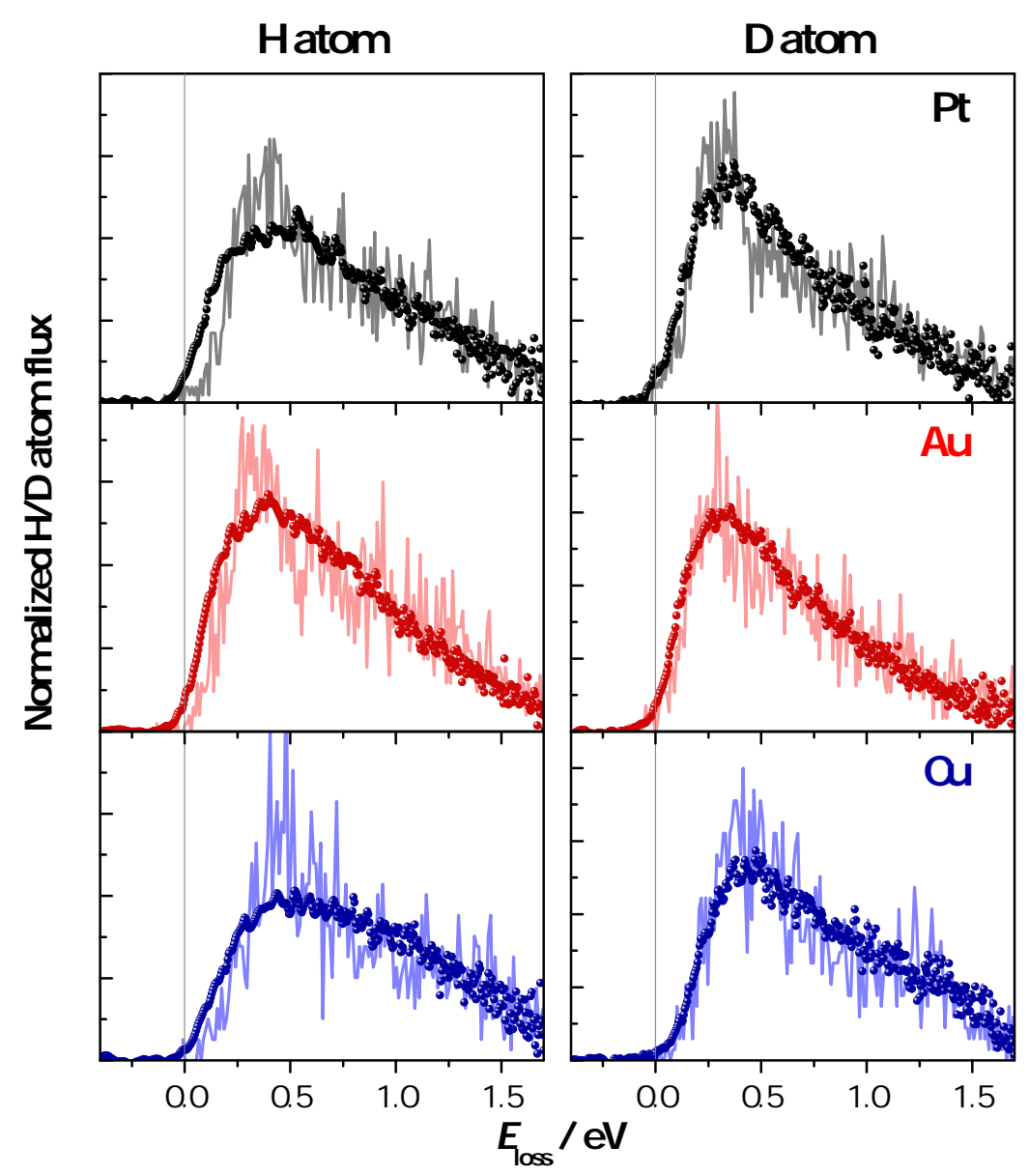

Figure 4.10: Comparison of experimental (black, red and blue circles) and simulated (grey, pale red, pale blue solid lines) energy loss spectra of $\mathrm{H} / \mathrm{D}$ atoms (left/right) obtained from $\mathrm{Pt}, \mathrm{Au}$ and $\mathrm{Cu}$. Incidence conditions: $E_{\mathrm{i}, \mathrm{H}}=1.92 \mathrm{eV}, E_{\mathrm{i}, \mathrm{D}}=1.87 \mathrm{eV}$, $\vartheta_{\mathrm{i}}=\vartheta_{\mathrm{s}}=45^{\circ} .127$ 


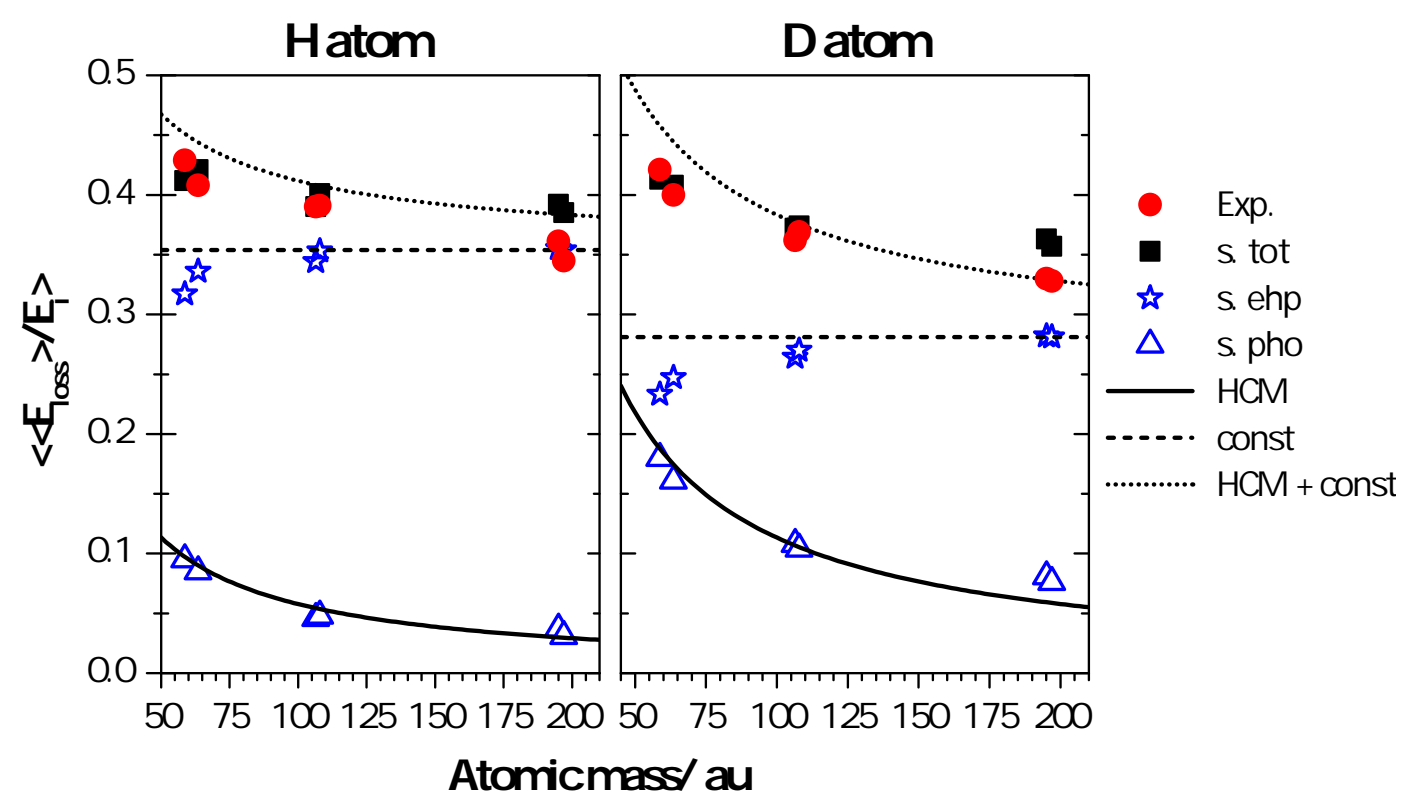

Figure 4.11: Comparison of relative average energy loss $\left\langle E_{\text {loss }}\right\rangle / E_{\mathrm{i}}$ of $\mathrm{H}$ (left) and D (right) plotted against the surface atomic mass experimentally obtained (filled red circles), EMT-LDFA-MD simulations (black filled squares, sim. tot) and a HCM model scaled up by a constant (black dotted line). Blue open symbols: Separation of energy loss into losses to phonons (triangles) and electronic excitations (ehp: stars), respectively. The energy loss predicted by the HCM model is illustrated as a solid black line, the constant as dashed black line. Experimental conditions: $\vartheta_{\mathrm{i}}=\vartheta_{\mathrm{s}}=45^{\circ} .127$ 
both effects on the average energy loss resulting from electronic excitation (open blue stars) and phonon excitation (blue open triangles) were added to the graph.

In general, the relative average energy loss decreases with increasing atomic mass. Determined experimental values and EMT-LDFA-MD simulations agree well, but for high atomic masses the theory generally overestimated the relative average energy loss.

Based on the theoretical model, the energy transfer to the surface is considered as the sum of two aforementioned effects: phonon and electron excitation. The energy loss to electron-hole-pairs (ehp: stars) decreases only slightly with decreasing atomic mass for both isotopes. In addition, ehp-excitation plays an exceedingly dominant role in the energy transfer process for both isotopes (effect slightly higher for D atoms). In contrast, the contribution of phonon excitation (triangles), which plays a subordinate role in the energy transfer process, increases with decreasing atomic mass and is enhanced for D compared to $\mathrm{H}$ atoms. According to theory, both contributions are nearly equally contributing for D atoms scattered from low atomic mass surfaces.

The phonon excitation contribution gained from MD-simulations can also be derived from a hard cube model (HCM) expanded to an attractive square well potential (here $W=2 \mathrm{eV}$, which is in the range of calculated H-Metal binding energies 130,136 ) and surface motion contributions as described by Equation 2.6 (black solid line). The mass $M$ was set to the atomic mass of the surfaces. Treating the ehp-excitation contributions as a constant fraction (dashed line), the simple HCM model shifted by this constant fraction (dotted black line) reproduce the trend of the experimentally determined values with surprisingly high accuracy, especially for high surface masses. However, the model clearly overestimates the resulting energy loss for the lighter $3 d$-metals which is significantly more pronounced for $\mathrm{D}$ atom scattering experiments and might be a result of the clearly false assumption of a constant ehp-excitation contribution.

The high accuracy of the HCM-model further supports the assumption that observed changes, when comparing the energy distributions, are predominantly a result of the varying masses although it is noteworthy that this effect is lower than would have been predicted by classical calculations. 


\subsection{Discussion}

Inelastic $\mathrm{H}$ and $\mathrm{D}$ atom scattering was performed on in total six different $f c c$ transition metals. Comparison of experimental data to theoretical calculations showed that in each case ehp-excitation dominates the inelasticity in the scattering event and allows for an efficient energy transfer for $\mathrm{H}$ and $\mathrm{D}$ atoms in order to consequently stick on a metal surface. The observed isotope effects are small and can be explained by a compensation effect of two strongly isotope dependent surface excitation processes: phonon excitation and ehp-excitation.

All energy and angular distributions are generally broad and are a result of multiple bounce scattering and surface penetration processes on the surface. Scattering dynamics are very similar for all investigated $f c c$ metal surfaces. Small deviations in the energy loss distribution could be traced back to the relative mass differences between the investigated metals and thus the efficiency of translational energy coupling to phonons. The efficiency of phonon excitation increases for lighter metals. Further, no significant effects of electronic structure or work function on energy transfer have been observed within this study.

At first sight, the observations made would stand in contrast to other previously published studies, which demonstrated strong differences in the energy transfer processes for different metal surfaces. For instance, investigations of hydrogen interaction with surfaces inducing chemicurrents on MIM devices revealed strong dependencies on the electronic structure. The changes result from electrons which are formed in the course of the $\mathrm{H}-\mathrm{H}$ surface recombination process. $45|46| 122 \mid 123$ In a separate study of vibrational relaxation probabilities of highly vibrationally excited NO on surfaces, $120 \mid 121$ vibrational relaxation efficiency was shown to depend on the work function of the surface due to formation of transient anionic species in charged energy transfers from the surface to the molecule. Energy transfers between hydrogen atoms and the studied metal surfaces is independent on the work function, which underlines that a formation of transient $\mathrm{H}^{-}$is irrelevant for effective energy transfers between $\mathrm{H}$ atoms and metal surfaces. However, the MD-simulation predict a small reduction in the electron excitation contribution for lighter metals. Non-adiabatic contributions in the simulation were calculated by electronic friction at the level of the local density friction approximation. The included friction coefficient is related to the background electronic density but reaches saturation for the metals investigated here. 137 Thus, a dependence of the electronic density on the energy transfer efficiency is not detectable within the margin of the experimental error and cannot be ruled out completely as all studied surfaces provide a similar electron density. 
Experiment and simulation agree exceedingly well for all investigated metals and experimental conditions, thus, the MD model is well suitable for the description of surface scattering dynamics. The simulation is further even able to predict the sticking probabilities of $\mathrm{H}$ and $\mathrm{D}$ atoms on the metal surfaces while the experimental determination remains still a challenge. 127 A consistent trend of an enhanced scattering flux (corresponding to a lower sticking probability) with increasing incidence energy could be observed for all experiments. Due to possible adjustment arrows and unavailability of out-of-plane measurements a reliable trend of the sticking probability with respect to the incidence angle and metal is not reasonable to propose. In the investigated energy range, theory predicts an uniformly high sticking probability of over $50 \%$ for $\mathrm{H}$ and $\mathrm{D}$ atoms impinging on a metal surface. The predictions show an enhanced sticking probability for lighter metals, as well as decreasing incidence energies and angles.

The theoretically determined sticking probabilities of $\mathrm{H}$ and $\mathrm{D}$ atoms on metals can further be well described by an universal sticking function, which depends on the incidence translational energy of the isotope $E_{\mathrm{i}}$, the incidence angle $\vartheta_{\mathrm{i}}$ and the atomic metal mass $M .127$

$$
S=\left(S_{0}+a \cdot E_{\mathrm{i}}+b \cdot M\right) \cdot\left(1-h\left(\vartheta_{\mathrm{i}}-c\right)\left(1-\cos \left(\vartheta_{\mathrm{i}}-c\right)^{d \cdot h\left(E_{\mathrm{i}}-e\right)\left(E_{\mathrm{i}}-e\right)}\right)\right)
$$

Here, $h(x)$ is a heavy step function. The applied variables are listed in the following Table 4.3

Table 4.3: Parameter for Equation 4.3 .

\begin{tabular}{|c|c|c|c|c|c|c|}
\hline Isotope & $S_{0}$ & $\begin{array}{c}a \\
/ \mathrm{eV}^{-1}\end{array}$ & $\begin{array}{c}b \\
/ u^{-1}\end{array}$ & $\begin{array}{l}c \\
/^{\circ}\end{array}$ & $\begin{array}{c}d \\
/ \mathrm{eV}^{-1}\end{array}$ & $\begin{array}{c}e \\
/ \mathrm{eV}\end{array}$ \\
\hline $\mathrm{H}$ & 1.081 & -0.125 & $-8.40 \cdot 10^{-4}$ & 28.88 & 1.166 & 0.442 \\
\hline $\mathrm{D}$ & 1.120 & -0.124 & $-1.20 \cdot 10^{-3}$ & 28.62 & 1.196 & 0.474 \\
\hline
\end{tabular}

According to Equation 4.3 a linear correlation of the sticking probability with the atomic mass and incidence energy is expected in the applied parameter space. For incidence angles close to the surface normal, the scattering probability is decreasing, which is in accordance with a penetration mechanism. 



\section{Ultra Thin Oxide Layer}

In this chapter, the first detailed inelastic $\mathrm{H} / \mathrm{D}$ atom scattering experiments from an insulator and ultra thin insulating oxide films will be presented.

Thin film materials with nanoscale thickness, frequently provide admirable material properties which strongly differ from the bulk material, which can include changes in the electrical, mechanical, optical, or thermal properties. To the best of knowledge, energy transfer processes between atoms/molecules and surfaces with a thickness in the nanoscale range have not yet been systematically studied in order to answer the question, whether such changes in the electronic and phonon structures have an impact on energy transfer processes. Such experiments may further lead to a deeper understanding of how atomic or molecular energy transfer to solids can be tailored by interface design on the atomic scale. 117

In order to perform surface structure specific studies of interest, in an initial first step, ultra thin films with strongly different Young's modules were chosen, as this might significantly change the phonon modes of the thin layer structures. Fig. 5.1 illustrates calculated phonon dispersion spectra of platinum (left panel) and $\alpha-\mathrm{Al}_{2} \mathrm{O}_{3}$ (right panel). The overlap of the phonon density of states between aluminum oxide and platinum is virtually negligible leading to strongly different Young's modules. 139|140 Therefore, scattering experiments on insulating aluminum oxide films with varied film thickness on platinum have been performed.

Very thin and closed films of these materials can be synthesized by atomic layer deposition (ALD) resulting in amorphous aluminum oxide layers 141 and polycrystalline platinum films. 142 ALD uses a chemical self-limiting growth mechanism which allows control over the film thickness and its composition. 143 In addition, due to the formation of chemical bonds between the interfaces, interface defects can be reduced in comparison to other surface preparation techniques, e.g. PLD (pulsed layer deposition). Furthermore, thermal stability and chemical stability of the chosen materials facilitates cleaning of samples in an UHV environment.

In the following, first results of $\mathrm{H}$ and $\mathrm{D}$ atom scattering from bulk single crystal surfaces of $\alpha-\mathrm{Al}_{2} \mathrm{O}_{3}$ and $\mathrm{Pt}(111)$ will be presented. Subsequently, $\mathrm{H}$ and D atom scattering results 


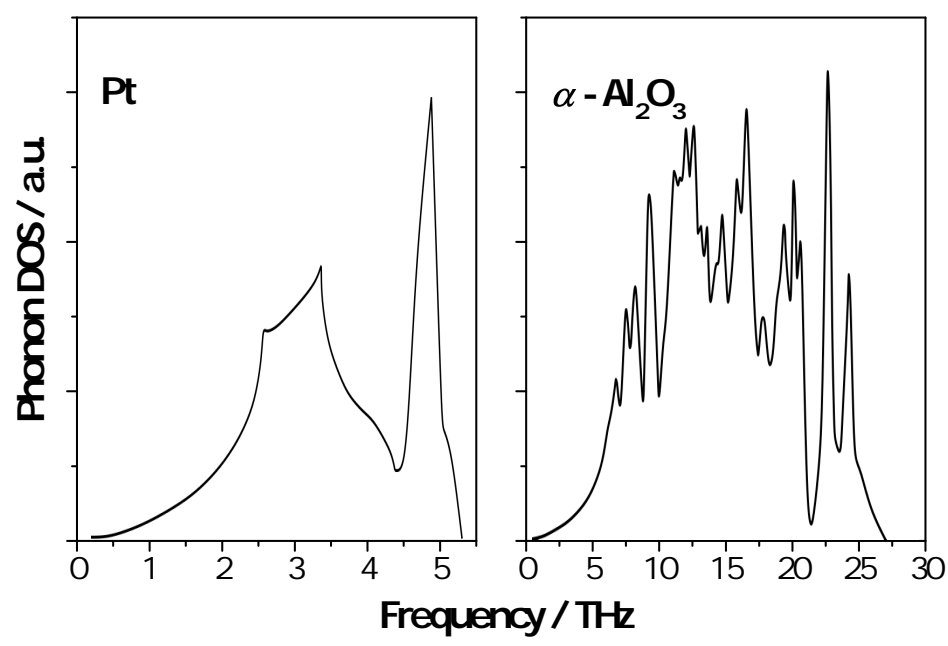

Figure 5.1: Calculated phonon dispersion spectra of platinum (left panel) and $\alpha-\mathrm{Al}_{2} \mathrm{O}_{3}$ (right panel). Data derived from Ohrlich et al. for platinum 138 and Wang et al. for $\alpha-\mathrm{Al}_{2} \mathrm{O}_{3} .139$

from ALD prepared aluminum oxide films with different thicknessses are discussed and compared to scattering results from bulk single crystal surfaces of $\alpha-\mathrm{Al}_{2} \mathrm{O}_{3}$ and $\operatorname{Pt}(111)$. A not fully closed monolayer (ML) of aluminum oxide was also prepared and investigated. For a deeper understanding of the scattering process, a $25 \%$ atomic oxygen covered $\mathrm{Pt}(111)$ surfaces was prepared and its scattering behavior subsequently compared to the not fully closed monolayer (ML) of aluminum oxide on Pt.

All experiments discussed in the following section were carried out with incidence angles of $\vartheta_{\mathrm{i}}=40^{\circ}$ and $55^{\circ}$ with respect to the surface normal and incidence energies of the incoming hydrogen atom beam of $E_{\mathrm{i}, \mathrm{H}}=1.92$ and $0.99 \mathrm{eV}$ at a surface temperature of $300 \mathrm{~K}$. Additionally, isotope effects were studied using a deuterium atom beam of similar incidence energies, $E_{\mathrm{i}, \mathrm{D}}=1.87$ and $0.94 \mathrm{eV}$. Angular distribution and energy distribution spectra presented here are normalized to the peak maximum and surface area, respectively. In Part 5.1, $\mathrm{H}$ and D atom scattering from the bulk materials, single crystal aluminum oxide with a (0001) surface cut $\left(\alpha-\mathrm{Al}_{2} \mathrm{O}_{3}(0001)\right)$ and single crystal platinum $(\mathrm{Pt}(111))$ are presented and discussed. These obtained results are subsequently compared in Section 5.2 to the experiments performed with ALD prepared aluminum oxide films on platinum with layer thickness down to the thinnest closed film of $1 \mathrm{~nm}$. In order to broaden the understanding of the properties of the oxide films, $\mathrm{H}$ and D scattering studies from 0.65 ML of $\mathrm{AlO}_{\mathrm{x}}$ on $\mathrm{Pt}$ and 0.25 ML of $\mathrm{O}$ on $\mathrm{Pt}(111)$ have been performed and are discussed in Section 5.3 . 


\section{1 $\mathrm{H}$ atom scattering from single crystalline surfaces}

A polar plot of representative angular distribution spectra of $\mathrm{H}$ atoms scattered from bulk single crystal $\alpha-\mathrm{Al}_{2} \mathrm{O}_{3}$ and $\mathrm{Pt}(111)$ are shown in Fig. 5.2 .

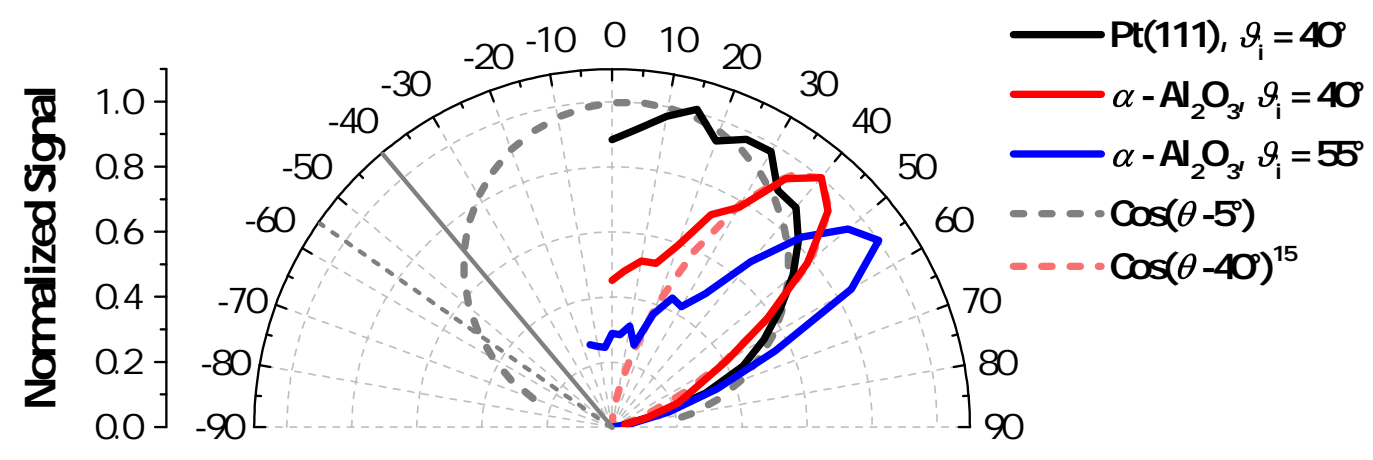

Figure 5.2: Representative angular distribution spectra of $\alpha-\mathrm{Al}_{2} \mathrm{O}_{3}$ single crystal (red solid line and blue line) and $\operatorname{Pt}(111)$ single crystal (black line) at $\vartheta_{\mathrm{i}}=40^{\circ}$ and $\vartheta_{\mathrm{i}}=55^{\circ}$ shown for an incidence energy of $E_{\mathrm{i}}=1.92 \mathrm{eV}$. Distributions of $\mathrm{Al}_{2} \mathrm{O}_{3}$ are narrow and peak at the specular angle $\vartheta_{\mathrm{s}}=40^{\circ}$ and $55^{\circ}$, respectively, whereas $\mathrm{Pt}(111)$ exhibits broad and structureless angular distributions. The grey solid and dotted lines indicate the incidence angles. The pale red and grey dashed lines represent cosine fits of the measured spectra obtained from $\alpha-\mathrm{Al}_{2} \mathrm{O}_{3}$ and $\mathrm{Pt}(111)$ single crystal surface for $\vartheta_{\mathrm{i}}=40^{\circ}$, respectively.

As aforementioned in Chapter 4 , the measured angular distributions of $\mathrm{Pt}(111)$ (black solid line) are very broad, structureless and weakly affected by modulations of the incidence angle within the investigated energy range. Here, an angular distribution of $\mathrm{Pt}(111)$ at $\vartheta_{\mathrm{s}}=40^{\circ}$ is shown. The obtained spectrum is in good agreement with a cosine distribution shifted by $5^{\circ}$ with respect to surface normal (grey dashed line).

In contrast, scattering experiments from single crystalline aluminum oxide sample at $\vartheta_{\mathrm{s}}=40^{\circ}$ (red line) exhibits a maximum scattering probability at about $40^{\circ}$ with respect to the surface normal. A narrow cosine distribution with a shift of $40^{\circ}$ (pale red dashed line) is well suitable for describing the basic shape of the angular dependent scattering distribution. However, a small portion of scattering signal at scattering angles close to the surface normal cannot be described by a single cosine function.

Further studies revealed a strong dependence of the angular distribution spectra on the incidence angle, e.g. for an incidence angle of $55^{\circ}$ (blue line) the spectrum peaks at $55^{\circ}$. In general, all recordings of angular distributions from $\alpha-\mathrm{Al}_{2} \mathrm{O}_{3}$ peak consistently at the specular angle independent of the incidence angle and energy or the isotope.

Representative translational energy distribution spectra of $\alpha-\mathrm{Al}_{2} \mathrm{O}_{3}$ (red circles) and $\operatorname{Pt}(111)$ (blue triangles) at incidence and scattering angles of $\vartheta_{\mathrm{i}}=40^{\circ}$ and $\vartheta_{\mathrm{s}}=50^{\circ}$ are 


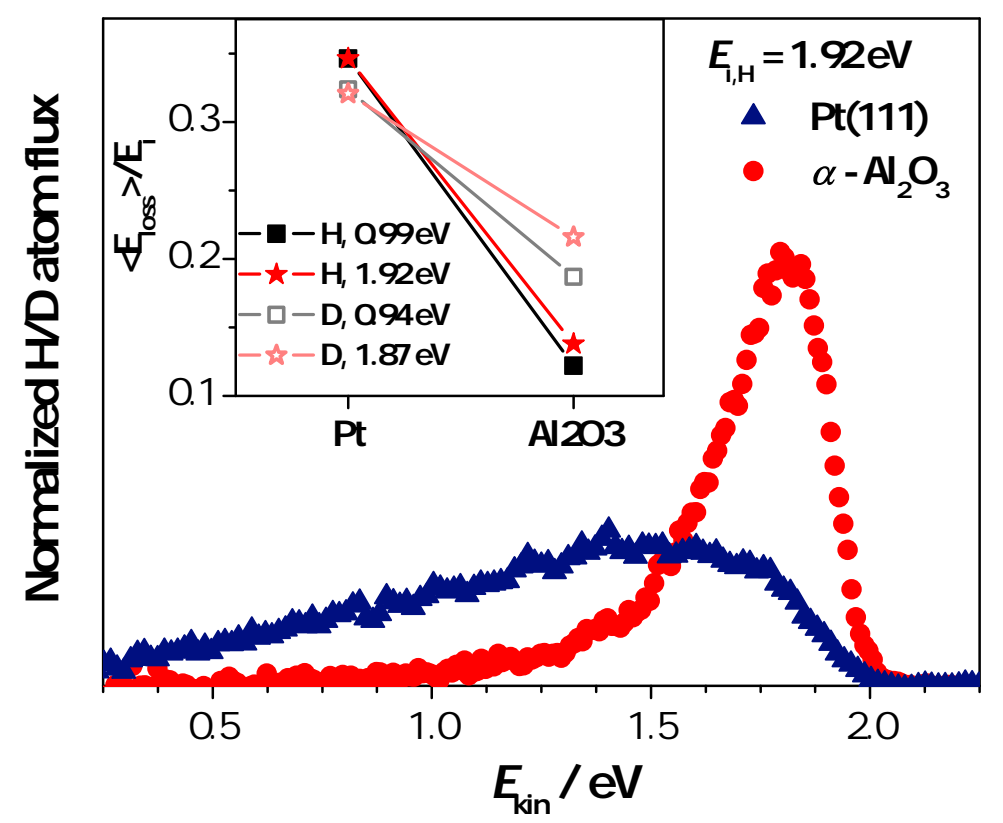

Figure 5.3: Comparison of energy distribution spectra of $\alpha-\mathrm{Al}_{2} \mathrm{O}_{3}$ (red circles) and $\mathrm{Pt}(111)$ (blue triangles) at $E_{\mathrm{i}}=1.92 \mathrm{eV}$. The energy probability distribution of $\mathrm{Pt}$ is broad and structureless, while the energy distribution spectrum of $\mathrm{Al}_{2} \mathrm{O}_{3}$ is less energy dispersed and peaks around $E_{\text {kin,max }}=0.94 \%$. Inset plots the normalized average energy loss, $\left\langle E_{\text {loss }}\right\rangle / E_{\mathrm{i}}$, of both samples against the incidence energy and investigated isotope: $E_{\mathrm{i}, \mathrm{H}}=0.99 \mathrm{eV}$ (filled black square), $E_{\mathrm{i}, \mathrm{H}}=1.92 \mathrm{eV}$ (filled red stars), $E_{\mathrm{i}, \mathrm{D}}=0.94 \mathrm{eV}$ (open black square), $E_{\mathrm{i}, \mathrm{D}}=1.87 \mathrm{eV}$ (open grey star).

presented in Fig. 5.3 .

The inset shows the observed normalized average energy losses $\left\langle E_{\text {loss }}\right\rangle / E_{\mathrm{i}}$ (stars) with respect to the incidence energy and isotope of both investigated samples. The energy distribution of $\operatorname{Pt}(111)$ is very broad with an average energy loss of $\left\langle E_{\text {loss }, \mathrm{H}}\right\rangle \approx 35 \%$ for $\mathrm{H}$ atom scattering. The average energy loss is nearly independent of incidence energy and only a weak isotope effect of $10 \%$ is observed $\left(\left\langle E_{\text {loss }, \mathrm{H}}\right\rangle /\left\langle E_{\text {loss }, \mathrm{D}}\right\rangle=1.1\right.$, compare Chapter 4 .

$\mathrm{H}$ atom scattering from aluminum oxide leads to a relative narrow probability distribution $(\mathrm{FWHM} \approx 300 \mathrm{meV}$ ) with low average energy loss (Overview see Table 5.1). 
Table 5.1: Determined average energy losses of $\mathrm{H} / \mathrm{D}$ atom scattering from $\alpha-\mathrm{Al}_{2} \mathrm{O}_{3}$.

\begin{tabular}{ccc} 
Isotope & $E_{\mathrm{i}} / \mathrm{eV}$ & $\left\langle E_{\text {loss }}\right\rangle / E_{\mathrm{i}}$ in $\%$ \\
\hline \hline $\mathrm{H}$ & 0.99 & 12 \\
$\mathrm{H}$ & 1.92 & 14 \\
$\mathrm{D}$ & 0.94 & 19 \\
$\mathrm{D}$ & 1.87 & 22
\end{tabular}

In general, the relative energy loss is more pronounced for D atom scattering which results in an isotope effect of approximately $40 \%$. The relative energy loss was further found to be dependent on the incidence energy, as for lower $E_{\mathrm{i}}$, a less pronounced normalized average energy loss was observed.
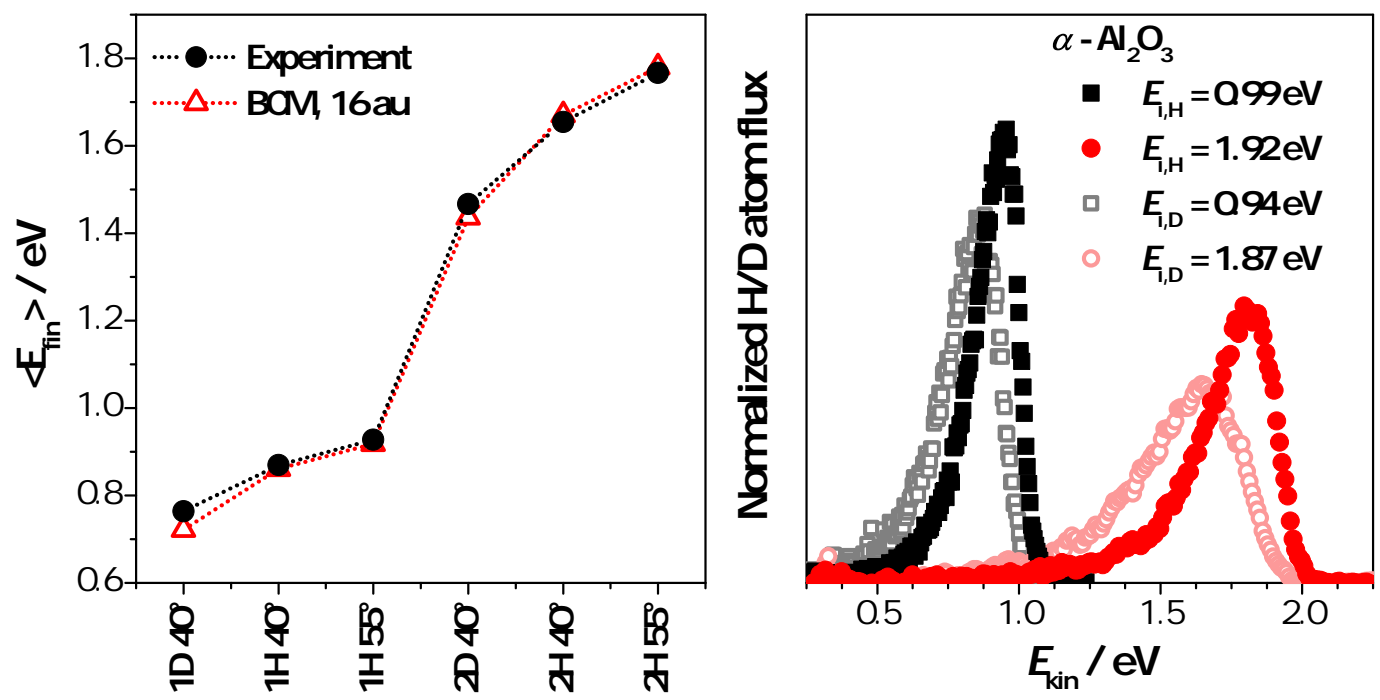

Figure 5.4: Left panel: Final average kinetic energy of the scattered atoms from $\alpha-\mathrm{Al}_{2} \mathrm{O}_{3}$ at the specular angle plotted against their incidence energy (rounded to 1 and $2 \mathrm{eV}$ ), isotope and angle conditions (black filled circles). The dependence on the incidence conditions can be described exceptionally well by a simple binary collision model (red open triangles) with a surface mass of $16 \mathrm{au}$. The dotted lines are used to guide the eye. Right panel: Comparison of $\mathrm{H}$ and $\mathrm{D}$ atom scattering from $\alpha-\mathrm{Al}_{2} \mathrm{O}_{3}$ at $E_{\mathrm{i}, \mathrm{H}}=1.92,0.99 \mathrm{eV}$ (red filled circles and black filled squares) and $E_{\mathrm{i}, \mathrm{D}}=1.87$, $0.94 \mathrm{eV}$ (pale red open circles and grey open squares). The relative energy loss is higher for D atom scattering. The experimental conditions are $\vartheta_{\mathrm{i}}=40^{\circ}, \vartheta_{\mathrm{s}}=50^{\circ}$.

The energy distribution of $\mathrm{H} / \mathrm{D}$ atoms scattered from single crystalline $\alpha-\mathrm{Al}_{2} \mathrm{O}_{3}$ are extremely sensitive to the applied experimental conditions. Right panel in Fig. 5.4 shows 
representative energy distribution spectra of $\mathrm{H}$ (filled red circles and filled black squares) and $\mathrm{D}$ atoms (open pale red circles and open grey squares) scattered from $\alpha-\mathrm{Al}_{2} \mathrm{O}_{3}$ at $\vartheta_{\mathrm{i}}=40^{\circ}$ and $\vartheta_{\mathrm{s}}=50^{\circ}$ with respect to the incidence energy and the isotope. The energy distributions of the presented experimental conditions all yield a peak at high scattering energies but significantly differ for the different isotope.

The left panel of Fig. 5.4 plots the experimentally observed average final kinetic energy of $\mathrm{H} / \mathrm{D}$ atoms scattered from $\alpha-\mathrm{Al}_{2} \mathrm{O}_{3}$ with respect to the incidence energy, isotope and angle conditions (black dots) at the specular scattering angle.

The dependence of the average final kinetic energy of $\mathrm{H} / \mathrm{D}$ atoms scattered at the specular angle can be describe exceedingly well by a simple binary collision model (BCM, red open triangles) as describe in Section 2.1.1. The theoretical final kinetic energies were calculated according to Equation 2.4 with an effective surface mass of 16 au.

The energy dependence and relative final kinetic energy of $\mathrm{H} / \mathrm{D}$ varies with incidence and scattering angle shown representatively for $\mathrm{H}$ atom scattering in Fig 5.5 .

Here, the energy distribution of $\mathrm{H}$ atoms scattered from $\alpha-\mathrm{Al}_{2} \mathrm{O}_{3}$ (red dots) is plotted at $E_{\mathrm{i}, \mathrm{H}}=1.92 \mathrm{eV}$ and $\vartheta_{\mathrm{i}}=40^{\circ}$ with respect to the scattering angle. With decreasing scattering angle the energy distribution gets broader and $\mathrm{H}$ atoms on average scatter with a lower kinetic energy.

Assuming a corrugated surface and predominantly specular scattering (e.g. binary hard sphere model, HSM: Eq. 2.7 or washboard model) the observed tendencies can be described by the extension of the BCM. The inset presents the relative average kinetic energy $\left\langle E_{\mathrm{kin}}\right\rangle / E_{\mathrm{i}}$ of scattered $\mathrm{H}$ atoms (filled black symbols) at various incidence conditions with respect to the total scattering angle $\vartheta=\vartheta_{\mathrm{i}}+\vartheta_{\mathrm{s}}$ calculated from the measured scattering angle $\vartheta_{\mathrm{s}}$ and incidence angle $\vartheta_{\mathrm{i}}$. The relative kinetic energy of the scattered atoms decreases with decreasing incidence and scattering angle. The red line presents the theoretical relative kinetic energy with respect to the incidence angle calculated by the extended BCM (effective surface mass $16 \mathrm{au}$ ) under the assumption that only specular scattering occurs. The model can simulate the experimentally obtained data fairly well for high scattering angles. Below $60^{\circ}\left(\vartheta_{\mathrm{s}}<30^{\circ}\right)$ the model however fails to describe the experimentally observed behavior.

The experimental observations show that the scattering dynamics from insulating $\alpha-\mathrm{Al}_{2} \mathrm{O}_{3}$ to metallic $\operatorname{Pt}(111)$ drastically differ from one another. The angular and relative energy distribution spectra of $\mathrm{Pt}(111)$ are very broad and nearly independent of the applied angle. In contrast, aluminum oxide exhibits quite narrow angular and energy distribution spectra which are strongly dependent on the incidence and scattering angle. These findings are in agreement with previous studies and results from Chapter 4. It was demonstrated that $\mathrm{H}$ 


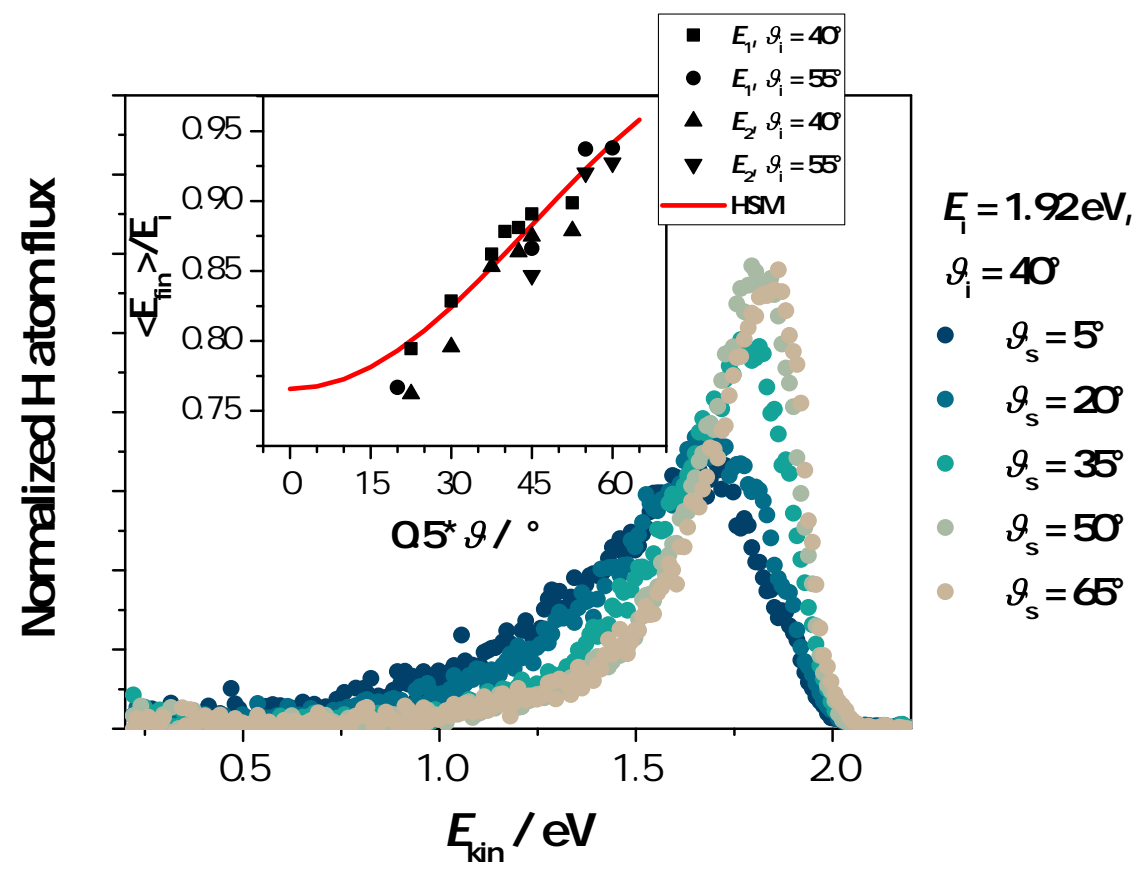

Figure 5.5: Energy distribution dependence of $\mathrm{H}$ atoms scattered from $\alpha-\mathrm{Al}_{2} \mathrm{O}_{3}$ (red dots) with respect to the half of the total scattering angle $\vartheta=\vartheta_{\mathrm{i}}+\vartheta_{\mathrm{s}}$ at $E_{\mathrm{i}, \mathrm{H}}=1.92 \mathrm{eV}$ and $\vartheta_{\mathrm{i}}=40^{\circ}$. With increasing scattering angle $\mathrm{H}$ atoms lose less kinetic energy. Inset: Obtained relative average final kinetic energy $\left\langle E_{\mathrm{kin}}\right\rangle / E_{\mathrm{i}}$ of scattered $\mathrm{H}$ atoms (filled black symbols) at different incidence energies and incidence angles (see legend, $\left.E_{1}=0.99 \mathrm{eV}, E_{2}=1.92 \mathrm{eV}\right)$ plotted against the theoretical specular scattering angle $0.5 \cdot\left(\vartheta_{\mathrm{i}}+\vartheta_{\mathrm{s}}\right)$. The red line shows the theoretical relative average final kinetic energy with respect to the incidence angle calculated by a hard sphere model (HSM) with a atomic mass of 16 au. 
atoms scattering from metals leads to a large translational energy loss and broad angular distribution caused predominantly by electronic excitation. From atomic-level simulations the broad angular distribution of $\mathrm{Pt}(111)$ can be attributed to multiple scattering events leading to to the displayed broad cosine distribution. 127

The narrow angular distribution of aluminum oxide and the conservation of incidence angle information is suggestive of a predominant direct scattering process. The strong dependence of the relative translational energy distribution of aluminum oxide on the incidence angle and energy conditions further supports this assumption. The average translational energy of scattered $\mathrm{H}$ atoms from $\alpha-\mathrm{Al}_{2} \mathrm{O}_{3}$ surface is about $87 \%$ (approx. $79 \%$ for $\mathrm{D}$ atom scattering). These results can mainly be explained by a simple binary collision model, which describes the scattering process as a single hard sphere collision (see Section 2.1.1. 34 Within this model, translational energy of the scattered atoms depends on the effective mass of the surface atoms. This simple model (with effective mass of $16 \mathrm{au}$ ) is able to predict the experimentally obtained relative final kinetic energies in dependence of the incidence and scattering conditions. In addition, the observed isotope effect can be explained well by the hard sphere model, and thus, can be attributed to phonon excitation. However, for low scattering angles $\left(\vartheta_{\mathrm{s}}<30^{\circ}\right)$, the model fails to describe the observed dependencies, which might be due to the increased presence of multiple bounce events or potential trapping desorption processes which are not considered in the theoretical model. In conclusion, the predicted effective mass demonstrates that the $\mathrm{H} / \mathrm{D}$ atoms scatter predominantly direct from a signal atom at the surface: $\mathrm{Al}(27 \mathrm{au})$ or $\mathrm{O}(16 \mathrm{au})$ with a higher effective mass. The observations might be explained by the fact that the $\mathrm{H} / \mathrm{D}$ atoms are quite small compared to the size of the surface atoms and exceedingly fast, so that the interaction time of the $\mathrm{H} / \mathrm{D}$ atoms with the surface is very short (fs-range). For this reason, the H/D atoms only interact with a single atom on the surface and are not effected by neighboring atoms on the surface.

However, a significant decrease of the overall scattering signal was observed for increasing incidence energy, indicative of a intermediate or high surface sticking probability of $\mathrm{H}$ and $\mathrm{D}$ atoms with increasing incidence energy.

Fig. 5.6 shows the relative $\mathrm{H}$ and $\mathrm{D}$ atom signal for $E_{\mathrm{i}, \mathrm{H}}=1.92 \mathrm{eV}$ (red line), $E_{\mathrm{i}, \mathrm{H}}=0.99 \mathrm{eV}$ (pale red line) and $E_{\mathrm{i}, \mathrm{D}}=1.87 \mathrm{eV}$ (blue line) at $\vartheta_{\mathrm{i}}=40^{\circ}$ integrated over all scattering angles.

Both the $\mathrm{H}$ and $\mathrm{D}$ atom signal decrease with increasing incidence energies. Thus, a higher incidence energy, which corresponds to a higher velocity on the molecular level, leads to an enhanced interaction with the surface. This might lead to the assumption of a deeper penetration into the surface, however, more sophisticated theoretical models are 


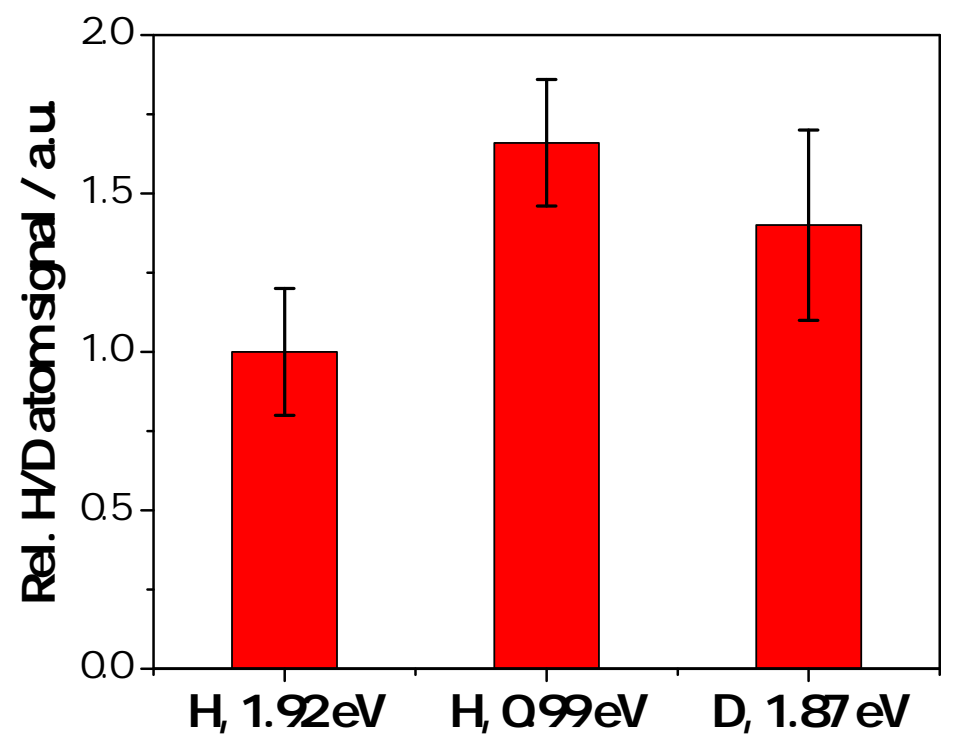

Figure 5.6: Relative $\mathrm{H}$ and $\mathrm{D}$ atom signal for $E_{\mathrm{i}, \mathrm{H}}=1.92 \mathrm{eV}$ (red line), $E_{\mathrm{i}, \mathrm{H}}=0.99 \mathrm{eV}$ (pale red line) and $E_{\mathrm{i}, \mathrm{D}}=1.87 \mathrm{eV}$ (blue line) at $\vartheta_{\mathrm{i}}=40^{\circ}$.

required for the full understanding of these interactions as such interactive processes are not considered within the hard-sphere model. 


\section{$5.2 \mathrm{H}$ tom scattering from ALD prepared thin layer $\mathrm{AlO}_{\mathrm{x}}$ surfaces}

In this section, $\mathrm{H}$ and $\mathrm{D}$ atom scattering results from $\mathrm{ALD}$ prepared aluminum oxide $\left(\mathrm{AlO}_{\mathrm{x}}\right)$ films of $1 \mathrm{~nm}$ and $50 \mathrm{~nm}$ thickness are presented and compared to the aforementioned results for single crystalline $\alpha-\mathrm{Al}_{2} \mathrm{O}_{3}$. Fig. 5.7 depicts the angular distribution of bulk single crystal $\alpha-\mathrm{Al}_{2} \mathrm{O}_{3}$ (black line), $\mathrm{AlO}_{\mathbf{x}}(50 \mathrm{~nm})$ (blue line) and $\mathrm{AlO}_{\mathbf{x}}(1 \mathrm{~nm})$ on $\mathrm{Pt}$ (red line) at $E_{\mathrm{i}}=1.92 \mathrm{eV}$ and $\vartheta_{\mathrm{i}}=40^{\circ}$.

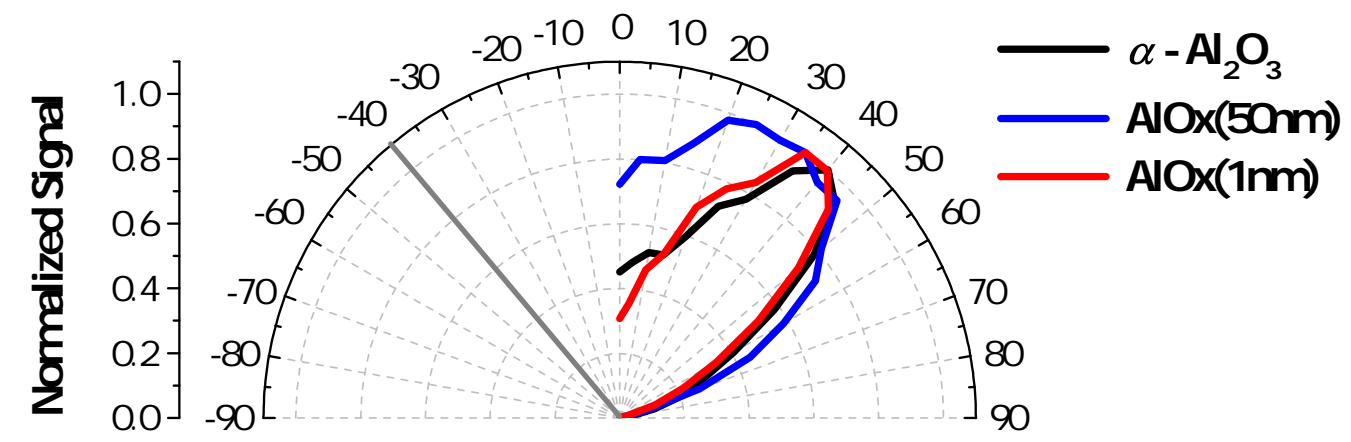

Figure 5.7: Comparison of angular distribution spectra of $\alpha-\mathrm{Al}_{2} \mathrm{O}_{3}$ single crystal (black line), $\mathrm{AlO}_{\mathrm{x}}(50 \mathrm{~nm})$ (blue line), and $\mathrm{AlO}_{\mathrm{x}}(1 \mathrm{~nm})$ on $\mathrm{Pt}$ (red line). The experimental conditions are $E_{\mathrm{i}}=1.92 \mathrm{eV}, \vartheta_{\mathrm{i}}=40^{\circ}$. The grey line indicates the incidence angle.

All angular distributions are basically unaffected by incidence energy and do not give rise to a measurable isotope effect but are strongly affected by the incidence angle. Spectra of bulk single crystal $\mathrm{Al}_{2} \mathrm{O}_{3}$ and $\mathrm{AlO}_{\mathrm{x}}(1 \mathrm{~nm})$ appear within the margin of error identical with narrow shape and a maximum at $\vartheta_{\mathrm{s}} \approx 40^{\circ}$, whereas the angular distribution of $\mathrm{AlO}_{\mathrm{x}}(50 \mathrm{~nm})$ is broadened and peaks at slightly lower $\vartheta_{\mathrm{s}}$.

The broadening of the angular distribution derived from $\mathrm{H}$ atoms scattered from the $50 \mathrm{~nm}$ ALD surface seem to be a result of the surface quality of the sample. Compared to the $1 \mathrm{~nm}$ thick $\mathrm{AlO}_{\mathrm{x}}$ sample which was grown on a thin platinum surface, the $50 \mathrm{~nm}$ thick $\mathrm{AlO}_{\mathrm{x}}$ film was grown directly on the Si waver. Comparison of AES and EDX measurements of the surface indicate that the synthesis leaded to an inhomogeneous $\mathrm{Al} / \mathrm{O}$ ratio within the $\mathrm{AlO}_{\mathrm{x}}$ layer. These observations might explain the angular distribution broadening as angular distributions in scattering experiments are sensitive to the quality of the ALD prepared surfaces, shown by Scheel et al. during their investigations of the negative ion fraction of positive charged molecules scattered from $\mathrm{AlO}_{\mathrm{x}}$ ALD samples. 144 Translational energy distribution spectra of $\mathrm{H} / \mathrm{D}$ atoms (red/pale red dots) scattered from a) single crystal $\left.\alpha-\mathrm{Al}_{2} \mathrm{O}_{3}, \mathbf{b}\right) \mathrm{AlO}_{\mathrm{x}}(50 \mathrm{~nm})$ and c) $\mathrm{AlO}_{\mathrm{x}}(1 \mathrm{~nm})$, are shown in Fig. 5.8 at 


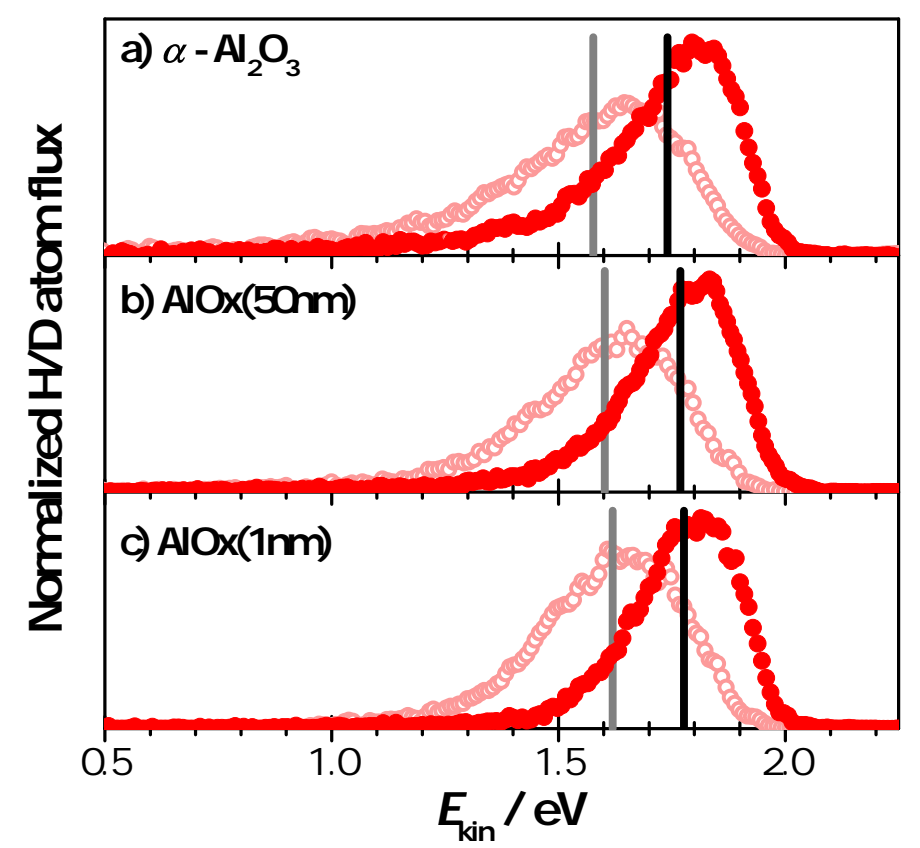

Figure 5.8: Comparative study of energy distribution spectra of $\mathrm{H}$ (red dots) and $\mathrm{D}$ atoms (pale red dots) scattered from a) $\alpha-\mathrm{Al}_{2} \mathrm{O}_{3}$ single crystalline, b) $\mathrm{AlO}_{\mathrm{x}}(50 \mathrm{~nm})$ and c) $\mathrm{AlO}_{\mathrm{x}}(1 \mathrm{~nm})$ at $E_{\mathrm{i}, \mathrm{H}}=1.92 \mathrm{eV}, E_{\mathrm{i}, \mathrm{D}}=1.87 \mathrm{eV}$ and $\vartheta_{\mathrm{i}}=40^{\circ}$. All spectra show a similarly shaped peak at about $1.82 \mathrm{eV}$ for $\mathrm{H}$ atom scattering and $1.66 \mathrm{eV}$ for $\mathrm{D}$ atom scattering. Only very small deviations are observed in the tail of the energy distribution towards lower energies. The black and grey bars mark the average kinetic energy of the scattered $\mathrm{H}$ and $\mathrm{D}$ atoms, respectively.

$E_{\mathrm{i}}=1.92 \mathrm{eV}, \vartheta_{\mathrm{i}}=40^{\circ}$ and $\vartheta_{\mathrm{s}}=50^{\circ}$. The black and grey bar mark the average kinetic energy of the scattered $\mathrm{H}$ and $\mathrm{D}$ atoms, respectively. In general, scattering of $\mathrm{H}$ atoms from these surfaces results in very similar energy distributions under all investigated experimental conditions.

A strong isotope effect of nearly $40 \%$ can be observed for all investigated aluminum oxide surfaces. The energy distribution of the surfaces all give rise to a pronounced peak towards higher kinetic energies, independent of the experimental conditions. However, a closer look on the comparison between the three investigated surfaces yields minor deviations. The most predominant deviations occur towards lower energies for ALD prepared $\mathrm{AlO}_{\mathrm{x}}$ compared to single crystal $\mathrm{Al}_{2} \mathrm{O}_{3}$. The determined average kinetic energies $\left\langle E_{\text {kin }}\right\rangle / E_{\mathrm{i}}$ and the isotope effect are listed in Table 5.2 
Table 5.2: Determined relative average kinetic energies $\left\langle E_{\mathrm{kin}}\right\rangle / E_{\mathrm{i}}$ and isotope effect 1 $\left\langle E_{\text {loss }, \mathrm{H}}\right\rangle /\left\langle E_{\text {loss }, \mathrm{D}}\right\rangle$ of $\mathrm{H} / \mathrm{D}$ atom scattering from $\alpha-\mathrm{Al}_{2} \mathrm{O}_{3}, \mathrm{AlO}_{\mathrm{x}}(50 \mathrm{~nm})$ and $\mathrm{AlO}_{\mathrm{x}}(1 \mathrm{~nm})$ on $\mathrm{Pt}$ at $E_{\mathrm{i}, \mathrm{H}}=1.92 \mathrm{eV}$ and $E_{\mathrm{i}, \mathrm{D}}=1.87 \mathrm{eV}$.

\begin{tabular}{lccc} 
Sample & $\left\langle E_{\mathrm{kin}, \mathrm{H}}\right\rangle / E_{\mathrm{i}, \mathrm{H}}$ & $\left\langle E_{\mathrm{kin}, \mathrm{D}}\right\rangle / E_{\mathrm{i}, \mathrm{D}}$ & Isotope effect in $\%$ \\
\hline \hline$\alpha-\mathrm{Al}_{2} \mathrm{O}_{3}$ & 0.86 & 0.78 & 36 \\
$\mathrm{AlO}_{\mathrm{x}}(50 \mathrm{~nm})$ & 0.90 & 0.84 & 38 \\
$\mathrm{AlO}_{\mathrm{x}}(1 \mathrm{~nm})$ & 0.89 & 0.82 & 39
\end{tabular}

While for both ALD prepared aluminium oxide surfaces comparable results were obtained, the derived data suggests a marginally, however significant, lower average kinetic energy for the experiments performed with single crystal $\alpha-\mathrm{Al}_{2} \mathrm{O}_{3}$ in comparison to the ALD prepared aluminum oxide surfaces.

Deviations in the energy distributions might be explained by differences in the composition of the surfaces. The applied ALD conditions yield amorphous aluminum oxide films with a lower atomic density and higher $\mathrm{O} / \mathrm{Al}$ ratio than single crystalline $\alpha-\mathrm{Al}_{2} \mathrm{O}_{3} .119,118$ According to literature, for an amorphous surface a broadening of angular distributions and energy distribtuions of the scattered atoms would be anticipated, whereas for crystalline surfaces of lower atomic density, the probability for direct scattering should increase as it directly correlates with the collision frequency. 146 Assuming a modification of the ALD prepared surfaces during the cleaning process migh explain the observed results. The ALD prepared surfaces were annealed during the cleaning process for more than $20 \mathrm{~h}$ at $700-750^{\circ} \mathrm{C}$. Crystallization of amorphous $\mathrm{ALD} \mathrm{AlO}_{\mathrm{x}}$ films on silicon substrates has been demonstrated at temperatures $\geq 800^{\circ} \mathrm{C}$, annealing within considerable shorter time periods as in the cleaning cycles perfomed on the investigated surfaces. $62[147,148$ Furthermore, amorphous $\mathrm{AlO}_{\mathrm{x}}$ on $\mathrm{NiAl}(100)$ has been shown to crystallize at $500^{\circ} \mathrm{C}$ (annealing times: $0.5-4 \mathrm{~h}$ ). 149 Experiments to investigate the degree of crystallinity of the investigated ALD sample were not performed in connection with the performed scattering experiments, thus, the degree of crystallization of the ALD samples would be worth investigating. However, the much lower atomic density and the higher $\mathrm{O} / \mathrm{Al}$ ratio of the ALD prepared $\mathrm{AlO}_{\mathrm{x}}$ films might sufficiently explain the deviation observed between the $\mathrm{ALD} \mathrm{AlO}_{\mathrm{x}}$ films and the $\alpha-\mathrm{Al}_{2} \mathrm{O}_{3}$ surface.

In conclusion, $\mathrm{H}$ atom energy losses on $\mathrm{AlO}_{\mathrm{x}}$ films with a thickness down to $1 \mathrm{~nm}$ (5 ML) appear quite similar to those values derived from experiments performed on a bulk single crystal aluminum oxide surface, which generally confirms that the ALD method can synthesis thin layer of aluminum oxid films of sufficiently high quality in order to perform successful atom scattering experiments. 
The insulating properties dominate the scattering process and no interaction between the insulating film and subsurface platinum layer was observed. Similar trends for the overall scattering signal in dependence of the incidence energy were observed for the ALD samples as for the single crystalline aluminum oxide surface. However, in subsequent studies observations were made indicating significantly lower overall scattering signals from $\mathrm{AlO}_{\mathbf{x}}(1 \mathrm{~nm})$ than from $\alpha-\mathrm{Al}_{2} \mathrm{O}_{3}$, which might be caused by a higher trapping probability for $\mathrm{AlO}_{\mathrm{x}}(1 \mathrm{~nm})$. Theoretical simulations of hydrogen atom scattering on metals observed that hydrogen atoms can penetrate into the metal surface which can cause a sticking of the hydrogen atom on the metal surface. $51|128| 150$ H atoms that penetrate into the $1 \mathrm{~nm}$ thick $\mathrm{AlO}_{\mathrm{x}}$ layer on platinum might not resurface and therefore would explain why no influence from the underlying platinum surface was observed.

Steinsiek et al.151 recently published a study of inelastic NO and CO scattering from ultra thin films of $\mathrm{Ag}$ on $\mathrm{Au}(111)$. These studies showed that the scattering behavior from dissimilar metal-metal films with a layer thickness of more than $3 \mathrm{ML}$ Ag behave principally identical to bulk Ag. However, in the range from 0 to $3 \mathrm{ML}$, drastic changes were observed for the final kinetic energy of the scattered molecules, leading to the conclusion that subsurface layers take part in the scattering process. According to this observation, an $\mathrm{AlO}_{\mathrm{x}}$ film of approximate $5 \mathrm{ML}$ on $\mathrm{Pt}$ is most likely too thick in order to observe an influence of the underlying platinum on the scattering dynamics. However, a thinner closed $\mathrm{AlO}_{\mathrm{x}}$ film cannot be prepared by ALD. Therefore, $\mathrm{H}$ atom scattering was performed on a not fully closed aluminum oxide layer on $55 \mathrm{~nm}$ Pt prepared by a single ALD cycle. 


\section{3 $\mathrm{H}$ atom scattering from oxide monolayer on $\mathrm{Pt}(111)$}

In this section, scattering results from an approximately $65 \%$ aluminum oxide covered Pt surface $\left(\mathrm{AlO}_{\mathrm{x}}(0.65 \mathrm{ML}) / \mathrm{Pt}\right)$ is studied in order to answer the question whether the platinum substrate has any influence on the $\mathrm{H} / \mathrm{D}$ atom scattering processes from aluminum oxide.

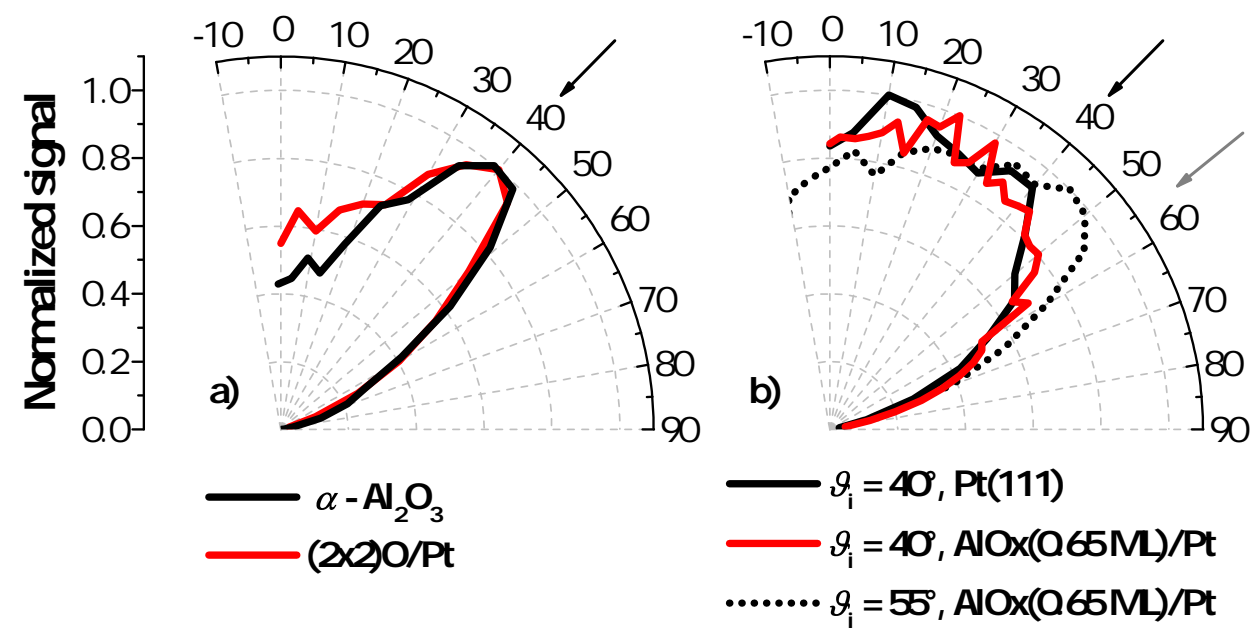

Figure 5.9: Angular distribution spectra of a) $\alpha-\mathrm{Al}_{2} \mathrm{O}_{3}$ single crystal (black dots) and $(2 \times 2) \mathrm{O} / \mathrm{Pt}(111)$ (red line) and b) $\mathrm{Pt}(111)$ single crystal (black dots) and $\mathrm{AlO}_{\mathbf{x}}(0.65 \mathrm{ML})$ on $\mathrm{Pt}$ (black line). Angular distribution spectra for surfaces in a) are narrow and peak at $\vartheta_{\mathrm{s}}=40^{\circ}$, whereas samples in $\mathbf{b}$ ) exhibit broad and structureless angular distribution spectra. The experimental conditions are $E_{\mathrm{i}, \mathrm{H}}=1.92 \mathrm{eV}$, $E_{\mathrm{i}, \mathrm{D}}=1.87 \mathrm{eV}$ and $\vartheta_{\mathrm{i}}=40^{\circ}$. An angular distribution of $\mathrm{AlO}_{\mathrm{x}}(0.65 \mathrm{ML})$ on $\mathrm{Pt}$ (black and grey line) at $\vartheta_{\mathrm{i}}=55^{\circ}$ is also shown. The grey solid $\left(\vartheta_{\mathrm{i}}=40^{\circ}\right)$ and dashed $\left(\vartheta_{\mathrm{i}}=55^{\circ}\right)$ lines mark the specular angle.

In order to gain deeper understanding of the scattering results, an atomic oxygen covered $\mathrm{Pt}(111)$ surface, $(2 \times 2) \mathrm{O} / \mathrm{Pt}$, with a lower oxygen coverage than the $\mathrm{AlO}_{\mathrm{x}}(0.65 \mathrm{ML}) / \mathrm{Pt}$ surface (corresponding to an oxygen coverage ratio of $2.6 \pm 0.4$ ), was investigated in a comparative series of scattering experiments.

Fig. 5.9 depicts the angular distributions for $\mathrm{H}$ atoms scattered from bulk single crystal $\mathrm{Al}_{2} \mathrm{O}_{3}$ (black dots) and 0.25 ML atomic oxygen on Pt(111) (red line) (left panel) as well as from bulk single crystal $\mathrm{Pt}(111)$ (black dots) and $\mathrm{AlO}_{\mathrm{x}}(0.65 \mathrm{ML}) / \mathrm{Pt}$ (grey line) (right panel). The experimental conditions were $E_{\mathrm{i}}=1.92 \mathrm{eV}$ and $\vartheta_{\mathrm{i}}=40^{\circ}$.

The angular distributions derived from $\mathrm{H}$ atom scattering from sample $(2 \times 2) \mathrm{O} / \mathrm{Pt}$, shown in a), are very similar for all investigated experimental conditions to angular distributions derived from $\mathrm{H}$ atom scattering from $\alpha-\mathrm{Al}_{2} \mathrm{O}_{3}$ and only small deviations are observed at 
scattering angles closer to the surface normal.

The angular distribution of $\mathrm{H}$ atoms scattered from $\mathrm{AlO}_{\mathrm{x}}(0.65 \mathrm{ML})$ has an identical shape as $\mathrm{Pt}(111), \mathbf{b})$. However, significant incidence angular dependence of angular distributions of $\mathrm{H}$ atoms scattered from $\mathrm{AlO}_{\mathrm{x}}(0.65 \mathrm{ML}) / \mathrm{Pt}$ are visible (grey line, $\vartheta_{\mathrm{i}}=55^{\circ}$ ).

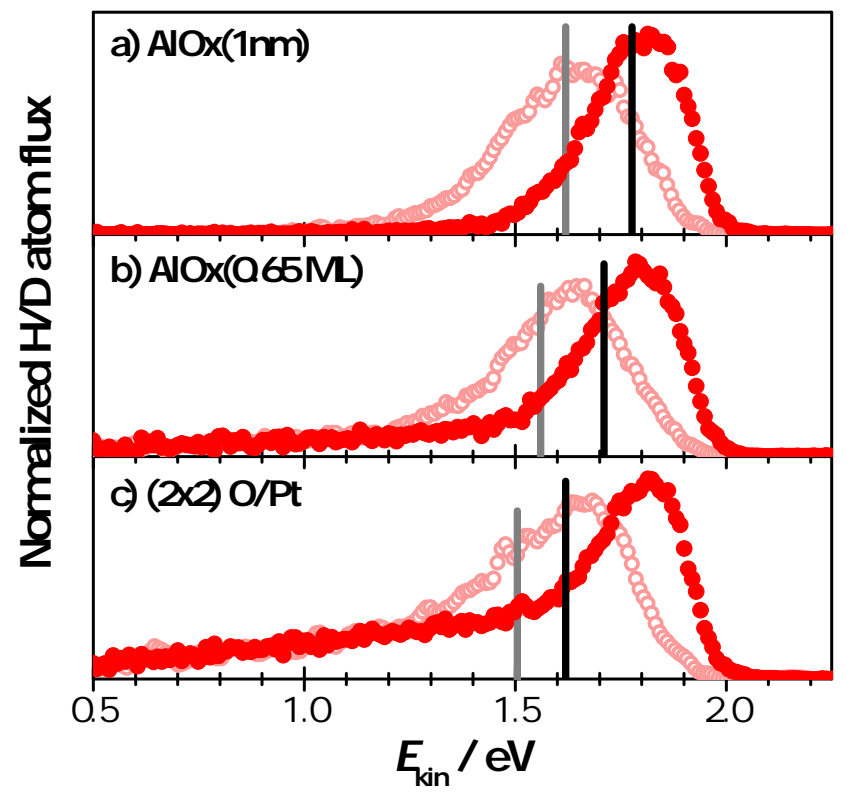

Figure 5.10: Energy distribution comparison of $\mathrm{H}$ (red dots) and $\mathrm{D}$ (red dots) atoms scattered from a) $\left.\mathrm{AlO}_{\mathrm{x}}(1 \mathrm{~nm}) / \mathrm{Pt}, \mathbf{b}\right) \mathrm{AlO}_{\mathrm{x}}(0.65 \mathrm{ML}) / \mathrm{Pt}$ and c) $(2 \times 2) \mathrm{O} / \mathrm{Pt}(111)$. All spectra show a seemingly identical sharp peak at $1.82 \mathrm{eV}$ for $\mathrm{H}$ atom scattering and $1.66 \mathrm{eV}$ for $\mathrm{D}$ atom scattering. In the energy distribution spectra the tail to lower kinetic energy differs. The grey line marks the average kinetic energy of the scattered $\mathrm{H}$ atoms. The experimental conditions are $E_{\mathrm{i}, \mathrm{H}}=1.92 \mathrm{eV}$ or $E_{\mathrm{i}, \mathrm{D}}=1.87 \mathrm{eV}, \vartheta_{\mathrm{i}}=40^{\circ}$ and $\vartheta_{\mathrm{s}}=50^{\circ}$.

In Fig. 5.10 energy distribution of $\mathrm{H} / \mathrm{D}$ atoms scattering from $\mathrm{AlO}_{\mathrm{x}}(1 \mathrm{~nm}) / \mathrm{Pt}$ a) is compared to $\left.\mathrm{AlO}_{\mathbf{x}}(0.65 \mathrm{ML}) / \mathrm{Pt} \mathbf{b}\right)$ and $\left.(2 \mathrm{x} 2) \mathrm{O} / \mathrm{Pt}(111) \mathbf{c}\right)$ for both isotopes with $\vartheta_{\mathrm{i}}=40^{\circ}$, $\vartheta_{\mathrm{s}}=50^{\circ}$ and $E_{\mathrm{i}, \mathrm{H}}=1.92 \mathrm{eV}$ (red dots) or $E_{\mathrm{i}, \mathrm{D}}=1.87 \mathrm{eV}$ (pale red dots).

The black and grey bar indicate the average energy loss for $\mathrm{H}$ and $\mathrm{D}$ atom scattering, respectively. The energy distribution of $\mathrm{H} / \mathrm{D}$ atoms scattered from $\mathrm{AlO}_{\mathrm{x}}(0.65 \mathrm{ML}) / \mathrm{Pt}$ and $(2 \times 2) \mathrm{O} / \mathrm{Pt}$ appear similar to the energy distribution of scattered H/D atoms from $\mathrm{AlO}_{\mathrm{x}}(1 \mathrm{~nm})$ : Energy distribution spectra strongly depend on the investigated isotope. In addition, all energy distributions exhibit a sharp peak at low energy loss independent of the experimental condition. However, the energy distributions of $\mathrm{H} / \mathrm{D}$ atoms scattered from $\mathrm{AlO}_{\mathrm{x}}(0.65 \mathrm{ML})$ and $(2 \mathrm{x} 2) \mathrm{O} / \mathrm{Pt}$ exhibit a noticeable tail for lower translational energies which intensifies with increasing incidence energies and decreasing incidence and 
scattering angles, leading to a reduction of the average kinetic energy and the isotope effect for lower oxide surface coverage. The isotope effects and relative average kinetic energies are listed in Table 5.3 .

Table 5.3: Determined relative average kinetic energies $\left\langle E_{\text {kin }}\right\rangle / E_{\mathrm{i}}$ and isotope effect $1-$ $\left\langle E_{\text {loss }, \mathrm{H}}\right\rangle /\left\langle E_{\text {loss }, \mathrm{D}}\right\rangle$ of scattered $\mathrm{H} / \mathrm{D}$ atom scattering at $E_{\mathrm{i}, \mathrm{H}}=1.92 \mathrm{eV}$ and $E_{\mathrm{i}, \mathrm{D}}=$ $1.87 \mathrm{eV}$.

\begin{tabular}{lccc} 
Sample & $\left\langle E_{\text {kin }, \mathrm{H}}\right\rangle / E_{\mathrm{i}, \mathrm{H}}$ & $\left\langle E_{\text {kin,D }}\right\rangle / E_{\mathrm{i}, \mathrm{D}}$ & Isotope effect in $\%$ \\
\hline \hline $\mathrm{AlO}_{\mathrm{x}}(1 \mathrm{~nm})$ & 0.89 & 0.82 & 39 \\
$\mathrm{AlO}_{\mathrm{x}}(0.65 \mathrm{ML})$ & 0.79 & 0.75 & 16 \\
$(2 \mathrm{x} 2) \mathrm{O} / \mathrm{Pt}$ & 0.76 & 0.74 & 8
\end{tabular}

This observation indicates a second scattering component with a higher relative energy loss which becomes more pronounced for $\mathrm{H}$ atom scattering from surfaces with lower oxygen coverage. This assumption could be supported, as the energy distribution of both investigated surfaces could be sufficiently well described by a linear combination of $\mathrm{H}$ atom scattering from pure $\mathrm{AlO}_{\mathrm{x}}$ and pure $\mathrm{Pt}(111)$ using the relative fractions of the respective components (see Figure 5.11).

Representative energy distribution spectra (black squares) of $\mathrm{H}$ atom scattering from $\mathrm{AlO}_{\mathrm{x}}(0.65 \mathrm{ML})$ (left panel) and $(2 \mathrm{x} 2) \mathrm{O} / \mathrm{Pt}$ (right panel) at $E_{\mathrm{i}, \mathrm{H}}=1.92 \mathrm{eV}, \vartheta_{\mathrm{i}}=40^{\circ}$ and $\vartheta_{\mathrm{s}}=35^{\circ}$ are presented. The shape of the observed energy distributions correlate with the sum of the shape of the energy distributions obtained from $\mathrm{H}$ atom scattering from pristine $\mathrm{Pt}(111)$ and pristine $\mathrm{AlO}_{\mathrm{x}}$.

In both panels the red and blue symbols correspond to energy distributions of $\mathrm{H}$ atom scattered from pure $\mathrm{AlO}_{\mathrm{x}}(1 \mathrm{~nm})$ and pure $\mathrm{Pt}(111)$, respectively, scaled to their scattering fraction occurring during $\mathrm{H}$ atom scattering from the monolayer surfaces $\mathrm{AlO}_{\mathrm{x}}(0.65 \mathrm{ML})$ and $(2 \times 2) \mathrm{O} / \mathrm{Pt}$. The grey line corresponds to a calculated linear combination using the respective fractions of both components, which is in very good agreement with the energy distribution curve observed for measurements on $\mathrm{AlO}_{\mathrm{x}}(0.65 \mathrm{ML}) / \mathrm{Pt}$. In case of $\mathrm{AlO}_{\mathrm{x}}(0.65$ $\mathrm{ML}) / \mathrm{Pt}, 67 \pm 2 \%$ scattering from the oxide and $33 \pm 2 \%$ scattering from the platinum surface is observed while for $(2 \times 2) \mathrm{O} / \mathrm{Pt}$ the scattering ratio from oxide to platinum surface is $52 \%: 48 \%( \pm 2 \%)$.

Energy distributions of both monolayer surfaces can be mathematically separated and assigned to a fraction of scattering events from oxide and a fraction of scattering events from platinum for all investigated experimental conditions. No other scattering dynamics could be observed. The ratio between scattering from oxygen and platinum of both surfaces is strongly dependent on the incidence and scattering angle and the incidence energy. 


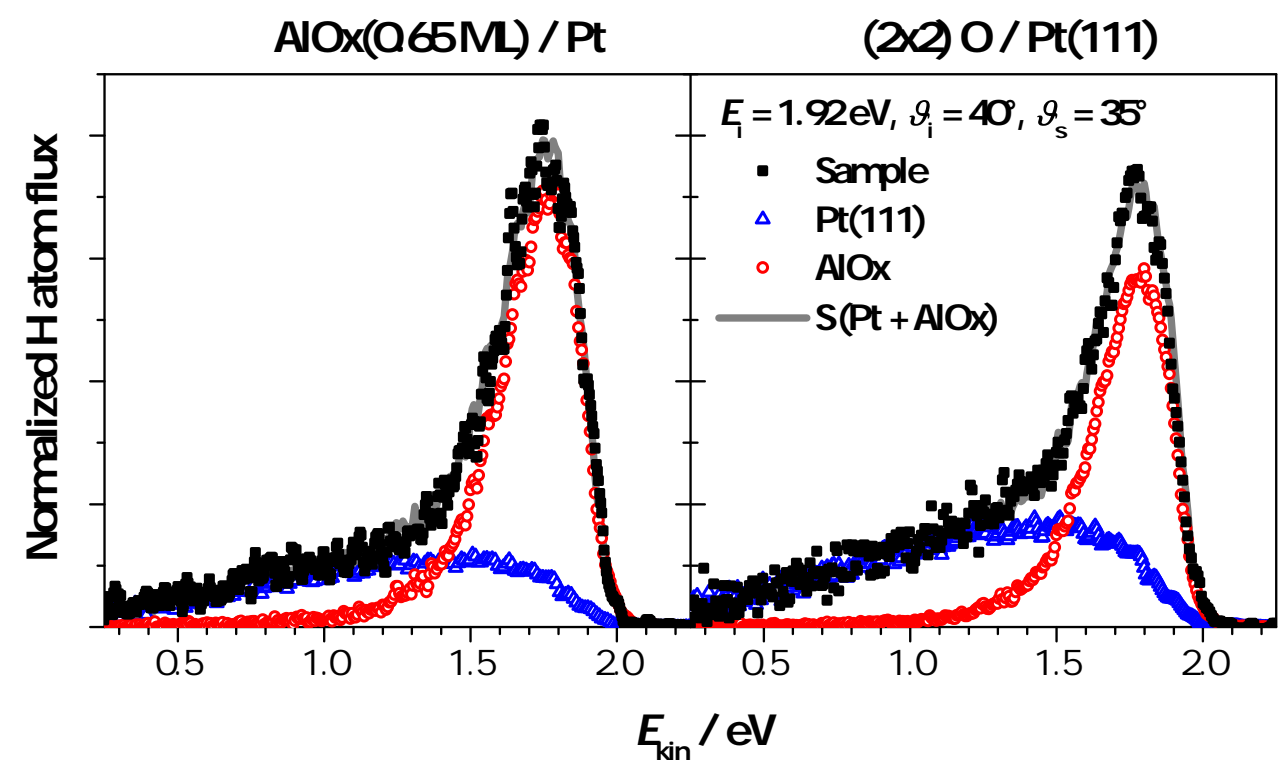

Figure 5.11: Energy distribution of $\mathrm{AlO}_{\mathrm{x}}(0.65 \mathrm{ML}) / \mathrm{Pt}$ (left panel, black squares) can be mathematically treated as linear combination of both $\mathrm{H}$ atom scattering from $\mathrm{AlO}_{\mathrm{x}}(1 \mathrm{~nm})$ (red circles) and $\mathrm{Pt}(111)$ (blue triangles). Right panel depicts ED of $(2 \mathrm{x} 2) \mathrm{O} / \mathrm{Pt}$ (black squares) and can be separated in energy distribution from $\mathrm{AlO}_{\mathrm{x}}(1 \mathrm{~nm})$ (red circles) and $\mathrm{Pt}(111)$ (blue triangles) as well. The sum of $\mathrm{AlO}_{\mathrm{x}}(1 \mathrm{~nm})$ an $\operatorname{Pt}(111)$ scattering experiments is marked as grey line. The experimental conditions are $E_{\mathrm{i}, \mathrm{H}}=1.92 \mathrm{eV}, \vartheta_{\mathrm{i}}=40^{\circ}$ and $\vartheta_{\mathrm{s}}=35^{\circ}$. The scattering fraction from platinum is higher for scattering from $(2 \times 2) \mathrm{O} / \mathrm{Pt}$.

Fig. 5.12 presents the angular distribution spectra (grey line) of $\mathrm{H}$ (a and $\mathbf{c}$ ) and $\mathrm{D}$ (b and $\mathbf{d}$ ) atoms scattered from $\mathrm{AlO}_{\mathbf{x}}(0.65 \mathrm{ML}) / \mathrm{Pt}$ sample $(\mathbf{a}$ and $\mathbf{b})$ and $(2 \times 2) \mathrm{O} / \mathrm{Pt}(111)$ (c and d) for $E_{\mathrm{i}, \mathrm{H}}=0.99 \mathrm{eV}$ or $E_{\mathrm{i}, \mathrm{D}}=0.94 \mathrm{eV}$ and $\vartheta_{\mathrm{i}}=40^{\circ}$.

All angular distributions can be separated into a fraction scattered from oxide (red) and a scattered fraction from Pt (blue). The sum of scattering fractions from oxide and platinum are shown in black and are identical with the experimentally observed angular distributions of $\mathrm{H} / \mathrm{D}$ atoms scattered from the not fully closed monolayer surfaces. Apart from this, scattering fractions from oxide and platinum for higher incidence energy of $E_{\mathrm{i}, \mathrm{H}}=1.92 \mathrm{eV}$ or $E_{\mathrm{i}, \mathrm{D}}=1.87 \mathrm{eV}$ are indicated as pale red and pale blue, respectively.

The angular distribution of $\mathrm{H} / \mathrm{D}$ atoms scattered from the well ordered $(2 \mathrm{x} 2) \mathrm{O} / \mathrm{Pt}$ can be fitted as a superposition of angular distributions from $\mathrm{H} / \mathrm{D}$ atoms scattered from $\mathrm{Al}_{2} \mathrm{O}_{3}$ and $\mathrm{Pt}$. The distribution of the fraction fraction from $\mathrm{Pt}$ of the $\mathrm{AlO}_{\mathrm{x}}(0.65 \mathrm{ML}) / \mathrm{Pt}$ surface is identical to the distribution from $\mathrm{H} / \mathrm{D}$ atom scattering from $\mathrm{Pt}(111)$, while the angular distribution of the scattering fraction from the oxide is much broader than the angular distribution of $\mathrm{H}$ atoms scattered bulk single crystal $\mathrm{AlO}_{\mathrm{x}}$. However, the angular distri- 
AOX(0.65 ML)/Pt

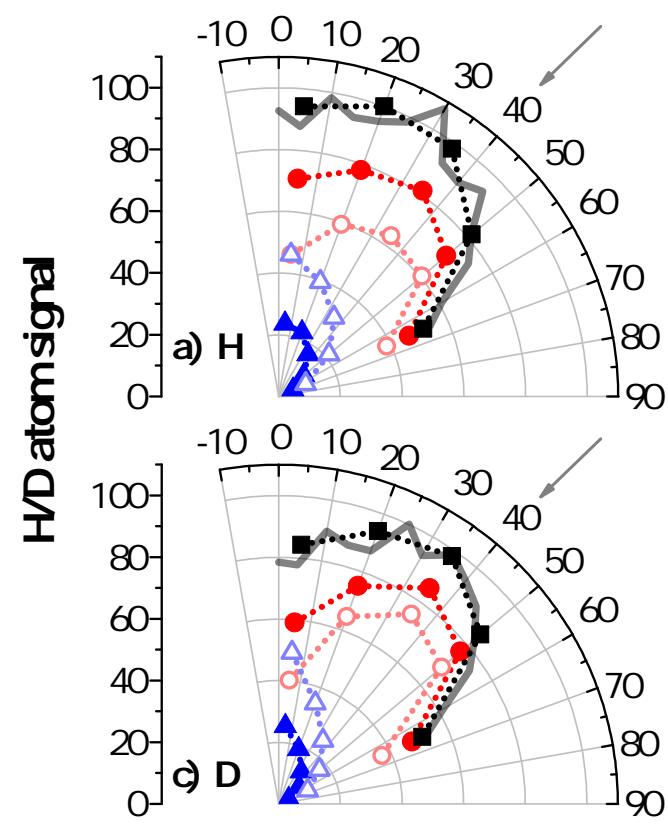

(2x2)0/ Pt(111)

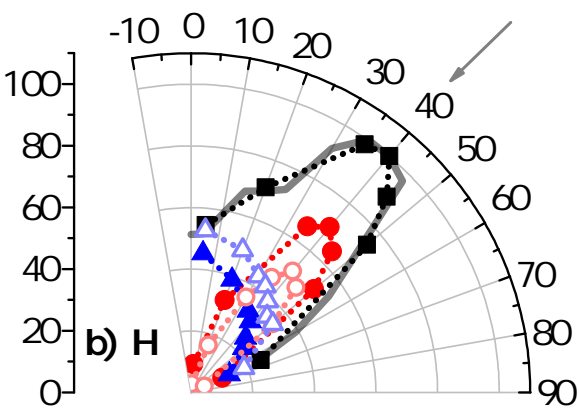

$-10$

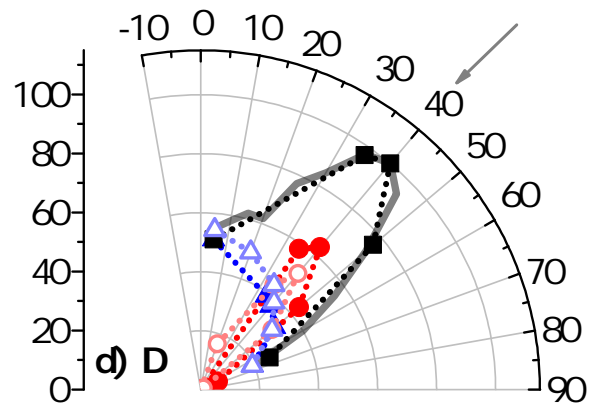

Figure 5.12: Angular distribution spectra (grey line) of $\mathrm{H}$ (a and c) and $\mathrm{D}$ (b and d) atoms scattered from $\mathrm{AlO}_{\mathrm{x}}(0.65 \mathrm{ML}) / \mathrm{Pt}$ sample (a and b) and $(2 \times 2) \mathrm{O} / \mathrm{Pt}(111)(\mathrm{c}$ and d) shown for $E_{\mathrm{i}, \mathrm{H}}=0.99 \mathrm{eV}$ or $E_{\mathrm{i}, \mathrm{D}}=0.94 \mathrm{eV}$ and $\vartheta_{\mathrm{i}}=40^{\circ}$. The arrow marks the specular angle. All angular distributions can be separated into a scattered fraction from oxides (red) and a scattered fraction from Pt (blue). The sum of oxide and platinum scattering fractions are shown in black. The angular distributions can be separated into the two scattering components for all investigated conditions. For $E_{\mathrm{i}, \mathrm{H}}=1.92 \mathrm{eV}$ or $E_{\mathrm{i}, \mathrm{D}}=1.87 \mathrm{eV}$ the oxide and platinum scattering fractions are indicated as pale red and pale blue, respectively. The Ox:Pt scattering ratio decreases with increasing incidence energy. At high incidence energy the $\mathrm{Ox}: \mathrm{Pt}$ scattering ratio change with the isotope.

bution of the scattering fraction from oxide behaves similar to the angular distribution from scattering from $\mathrm{AlO}_{\mathrm{x}}(50 \mathrm{~nm})$. The broadening of the angular distribution assigned to the scattering fraction from the oxide might stem from the low quality and roughness of the amorphous $\mathrm{AlO}_{\mathrm{x}}(0.65 \mathrm{ML}) / \mathrm{Pt}$ film.

The scattering fractions from oxide and platinum change with incidence energy. In order to compare the Ox:Pt scattering ratio between the different surfaces, the angular distributions were fitted by cosine distributions in order to estimate the overall scattering signal in plane of the incidence H/D atom beam. The scattering fraction from the oxide of the $\mathrm{AlO}_{\mathrm{x}}(0.65 \mathrm{ML}) / \mathrm{Pt}$ surface decreases with increasing incidence energy from approximately $78 \%$ to approximately $54 \%$ for $\mathrm{H}$ atom scattering (D atom: $\sim 78 \%$ to $\sim 57 \%$ ). In case of 
(2x2)O/Pt, the scattering fraction from oxide diminishes from about $40 \%$ to about $20 \%$ with increasing energy for $\mathrm{H}$ atom scattering (D atom: $\sim 30 \%$ to $\sim 20 \%$ ).

In conclusion, the Ox:Pt scattering ratio decreases with increasing incidence energy, leading to a higher scattering fraction from platinum. For higher kinetic energies, in general a deeper penetration into the surface by the H/D atom can be anticipated. Thus, the probability of $\mathrm{H} / \mathrm{D}$ atom scattering from lower Pt subsurfaces increases. The $\mathrm{Ox}: \mathrm{Pt}$ ratio depends not only on the atomic velocity but also on the isotope. $\mathrm{H}$ and $\mathrm{D}$ atom scattering have a similar Ox:Pt scattering ratio for lower incidence energies. However, significant higher variations of the $\mathrm{Ox}$ :Pt ratio were observed for $\mathrm{H}$ atom scattering with increasing incidence energy compared to D atom scattering. Comparing the oxide scattering fraction of both surfaces, the ratio is estimated to $2.7 \pm 0.1$, which would correlate very well to the oxygen coverage ratio of both surfaces (2.6) estimated by AES (Section 3.7.3), indicating a linear scaling of the oxide scattering fraction with oxygen coverage.

The Ox:Pt scattering ratio depends on the incidence energy and isotope indicating that a significant sticking probability exists on both surfaces $\mathrm{Pt}$ and $\mathrm{AlO}_{\mathrm{x}}$. Taking the theoretically predicted scattering probability of $\mathrm{H}$ and $\mathrm{D}$ atoms on $\mathrm{Pt}(111)$ into account (Section 4.5 , the sticking probability on $\mathrm{AlO}_{\mathrm{x}}$ monolayer can be calculated from the observed Ox:Pt flux ratio. Table presents the obtained sticking probabilities.

Table 5.4: Estimated sticking probabilities of $\mathrm{AlO}_{\mathrm{x}}$ monolayer of sample IV at $\vartheta_{\mathrm{i}}=40^{\circ}$ and $T_{s}=300 \mathrm{~K}$.

\begin{tabular}{lcccc} 
Surface & $\mathrm{H}, 0.99 \mathrm{eV}$ & $\mathrm{H}, 1.92 \mathrm{eV}$ & $\mathrm{D}, 0.94 \mathrm{eV}$ & $\mathrm{D}, 1.87 \mathrm{eV}$ \\
\hline \hline $\mathrm{AlO}_{\mathrm{x}}(0.65 \mathrm{ML}) / \mathrm{Pt}$ & $0.79 \pm 0.1$ & $0.87 \pm 0.07$ & $0.71 \pm 0.14$ & $0.85 \pm 0.08$ \\
$\mathrm{Pt}(111)$ & 0.78 & 0.66 & 0.76 & 0.63
\end{tabular}

The sticking probability of monolayer $\mathrm{AlO}_{\mathrm{x}}$ on Pt increases with the incidence energy and seems to be reduced for $\mathrm{D}$ atom scattering. These values are quite high and might further be influenced by the sublayer platinum. An estimation of the sticking probability on the thicker alumina films was not possible due to scaling issues. However, the experimental observations of the overall H/D atom flux would support such a high sticking probability: The sticking probability is enhanced for higher incidence energies and is universally smaller for $\mathrm{D}$ atom scattering. 


\subsection{Discussion}

For the first time, inelastic $\mathrm{H} / \mathrm{D}$ atom scattering from ALD prepared ultra thin aluminum oxide films on $\mathrm{Pt}$ were performed and compared to scattering results from the single crystalline bulk materials. For aluminum oxide, $\mathrm{H}$ and $\mathrm{D}$ atom scattering behavior can be explained within a simple binary collision model, and thus, attributed primarily to adiabatic phonon excitation. In contrast, scattering processes at platinum surfaces are predominantly influenced by non-adiabatic electronic excitation. ALD prepared surfaces down to the thinnest examined closed layer of aluminum oxide on platinum behave nearly identical to bulk single crystal aluminum oxide. Thus, no influence of the underlying platinum substrate was observed.

Energy distributions of $\mathrm{H} / \mathrm{D}$ atom scattering from a not fully closed monolayer of aluminum oxide on platinum can be separated into two scattering components: Scattering from aluminum oxide and scattering from platinum. No further scattering processes are proposed that may have resulted from synergistic effects of both materials. H/D atom scattering ratios from oxide to platinum depend on the initial velocity and isotope of the incoming atom. The oxidized platinum surface with atomic oxygen coverage of $25 \%$ shows similar $\mathrm{H} / \mathrm{D}$ atom scattering dynamics as the $\mathrm{AlO}_{\mathrm{x}}(0.65 \mathrm{ML}) / \mathrm{Pt}$ surface. Scattering results from oxidized platinum can be separated into a linear combination of $\mathrm{H} / \mathrm{D}$ atom scattering from oxide and platinum as well. Scattering experiments on both oxide films show basically identical energy distributions of the direct scattering component. The fraction of $\mathrm{H}$ atom scattering from either oxide or platinum scales nearly linear with relative oxygen coverage. No mass effects of the different surfaces influencing the energy loss of the scattering component derived from measurements on the oxide were observed. This indicates that $\mathrm{H} / \mathrm{D}$ atoms predominantly scatter directly from the oxygen atoms on oxide surfaces. 


\section{Graphene on $\mathrm{Ni}(111)$}

As aforementioned in the introduction, hydrogen interactions with graphene surfaces are currently a highly discussed topic in surface science. $15[19$ One important aspect of the $\mathrm{H}$-graphene interaction is the nature of the initially $\mathrm{H}$ atom trapping and sticking process on graphene surfaces. Thus, we set out to further explore the reaction dynamics on different graphene surfaces using the Rydberg atom tagging technique.

For such experiments, hydrogen atom collision on a graphene surface can induce a $\mathrm{C}-\mathrm{H}$ bond formation (Fig. 6.1 left).
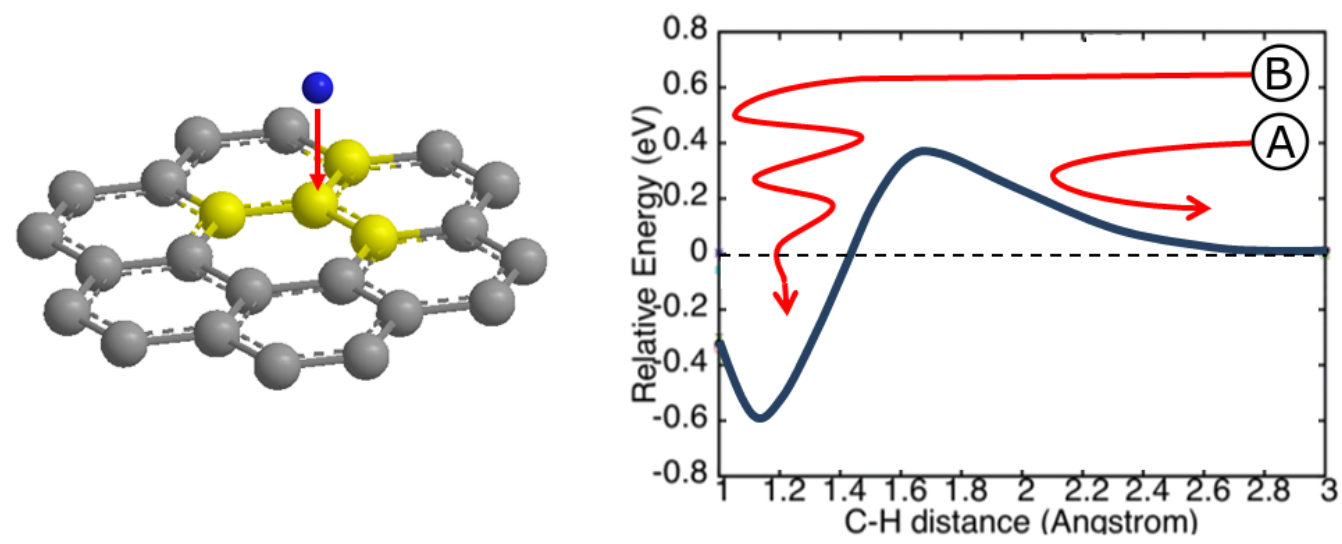

Figure 6.1: Left: Model structure of $\mathrm{C}-\mathrm{H}$ bond formation on graphene. Right: Illustration of a calculated PEC for the reaction of pyrene with hydrogen atom as a function of the distance between $\mathrm{H}$ and the nearest carbon atoms. Reprinted with permission from 152. Copyright 2012 American Chemical Society.

In order to form a $\mathrm{C}-\mathrm{H}-\sigma$ bond, the conjugated $\pi$-system of graphene has to be locally destroyed. This process involves the partial transition from $\mathrm{sp}^{2}$ to $\mathrm{sp}^{3}$ hybridization which results in a distortion of the $\mathrm{C}-\mathrm{C}$ bonds in the graphene layer. The need to break the conjugated $\pi$-system of graphene in the $\mathrm{C}-\mathrm{H}$ formation and therefore induced reconstruction of the graphene surface gives rise to an adsorption barrier (PEC: Fig. 6.1. right panel). Two scattering scenarios would be expected:

A: If a hydrogen atom with an incidence energy below the adsorption barrier strikes the surface it can be directly scattered back from the surface. 
B: If the hydrogen atom overcomes the barrier, a $\mathrm{C}-\mathrm{H}$ bond can be formed and the $\mathrm{H}$ atom might subsequently be trapped on the graphene surface.

Quantum scattering calculations based on DFT predict for $\mathrm{H}$ atoms striking a graphite surface (calculated barrier $0.2 \mathrm{eV}$ ) at normal incidence with energies above the barrier a significant trapping probability. For example, at $E_{\mathrm{i}}=0.3 \mathrm{eV}$ an initial sticking probability of approximately $80 \%$ was estimated. 153 These studies further predict that after about $100 \mathrm{fs}$, most of the trapped $\mathrm{H}$ atoms scatter back into the gas phase. Thus, a scattering component might be visible in $\mathrm{H}$ atom scattering experiments on graphene resulting from $\mathrm{H}$ atoms crossing the adsorption barrier. Theoretical simulations investigating free-standing graphene determined barrier values for the $\mathrm{C}-\mathrm{H}$ bond formation of $0.13-0.44 \mathrm{eV} .152 \mid 154$ Adsorption of graphene on different metal surfaces changes the electronic structure of the graphene layer, and thus, does alter the reaction process. For instance, graphene on $\mathrm{Pt}(111)$ is weakly bound while on $\mathrm{Ni}(111)$ a strongly bound $(1 \mathrm{x} 1)$ graphene layer can be produced, which significantly disturbs the conjugated $\pi$-system of the graphene layer (Chapter 2.2.3); effectively lowering the adsorption barrier for the $\mathrm{C}-\mathrm{H}$ bond formation step. Previous investigations of hydrogenation on (1 x 1) graphene on $\mathrm{Ni}(111)$ by helium-3 diffraction and spin-echo spectroscopy revealed a reduced activation barrier of $(1 \times 1) \mathrm{Gr} / \mathrm{Ni}(111)$ for the zero-coverage adsorption process of $E_{\mathrm{a}}=89 \pm 7 \mathrm{meV}$, which indicates a drastically reduced barrier of $\mathrm{Gr} / \mathrm{Ni}(111)$ compared to free-standing graphene. 155

The interaction strength between the graphene and $\mathrm{Ni}(111)$ surface can further be tuned by changing the graphene modification on $\mathrm{Ni}(111)$ which might influence the adsorption barrier height. 89

Initiated by previously performed studies on inelastic hydrogen atom scattering from quasi free-standing graphene on $\mathrm{Pt}(111)$ by our group (next section), in this thesis, the influence of the graphene configuration on a $\mathrm{Ni}(111)$ substrate and thus the strength of the graphene-substrate interaction on the reaction dynamics of hydrogen on graphene will be examined. Therefore, inelastic hydrogen atom scattering from two different graphene modifications on $\mathrm{Ni}(111)$, with significantly different interaction strength between the graphene film and metal substrate, was performed (surface preparation and characterization see Chapter 3.7.4. 


\subsection{Previous studies of Graphene on $\operatorname{Pt}(111)$ and theoretical calculations}

Inelastic $\mathrm{H}$ atom scattering experiments from a graphene surface on $\mathrm{Pt}(111)$ with the Rydberg atom tagging method (see Chapter 3) were performed by H. Jiang. 29 This chapter provides a brief summary of these investigations.

Translational energy and angular distributions of the scattered hydrogen atoms were measured with respect to the total incidence energy $\left(E_{\mathrm{i}}=0.99-3.31 \mathrm{eV}\right)$, incidence angle and the surface temperature.

Changing the incidence energy $E_{\mathrm{i}}$ or the incidence angle $\vartheta_{\mathrm{i}}$ at one incidence energy causes a change of the normal incidence energy $E_{\mathrm{n}}$ according to Equation 6.1.

$$
E_{\mathrm{n}}=E_{\mathrm{i}} \cdot \cos ^{2}\left(\vartheta_{\mathrm{i}}\right)
$$

Investigation of the scattering dynamics with respect to the normal incidence energy further revealed strong changes of the energy distribution spectra.

Figure 6.2 shows six representative two-dimensional (2D) translational energy and scattering angular distributions of $\mathrm{H}$ atoms scattered from graphene on $\operatorname{Pt}(111)$ at $E_{\mathrm{i}}=1.92 \mathrm{eV}$ with respect to the normal incidence energy $E_{\mathrm{n}}$ by changing the incidence angle (specular angle indicated by red arrows).

The distributions exhibit two different scattering components with respect to the normal incidence energy. At low normal incidence energies (left upper panel), a fast component with low energy loss can be observed near the specular scattering angle with narrow energy and angular distribution. With increasing normal incidence energy (left $\rightarrow$ right), this quasi-elastic peak starts to disappear and another component with a much longer time-of-flight emerge. This slow component has a larger energy loss $(40-50 \%$ of its initial translational energy) and is preferentially located around a scattering angle of $30^{\circ}$. Furthermore, an increase of the ratio between the slow and fast component was observed for increasing total incidence energy, normal incidence energy (decreasing incidence angle) and surface temperature, respectively. The differences in the two components indicate two different scattering dynamics which occur simultaneously on the graphene surface. 

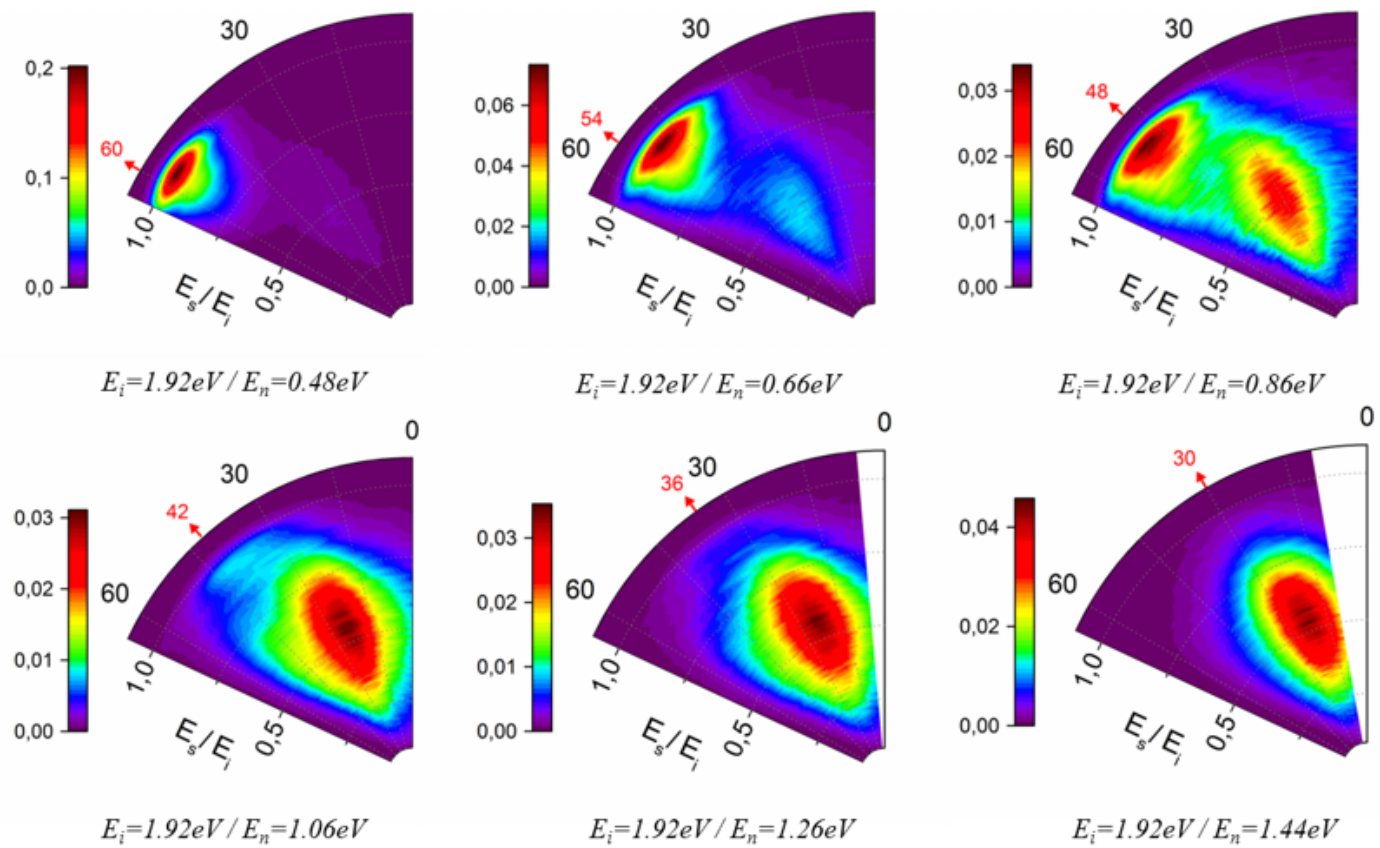

Figure 6.2: Two-dimensional translational energy and scattering angle distributions of $\mathrm{H}$ atoms scattered from graphene on $\mathrm{Pt}(111)$ at $E_{\mathrm{i}}=1.92 \mathrm{eV}$ with respect to the incidence angle (normal incidence energy $E_{\mathrm{n}}$ ). The 2D tanslational energy and angular distribution spectra present a measured data-set of the $\mathrm{H}$ atom scattering probability (color coding) with respect to the translational energy of the scattered $\mathrm{H}$ atoms normalized to the incidence energy $\left(E_{\mathrm{s}} / E_{\mathrm{i}}\right.$, radial axis) and the measured scattering angle (polar axis). The incidence angle and scattering angle are measured with respect to the surface normal $\left(\vartheta=0^{\circ}\right)$. The red arrows display the specular scattering angle. Radial axis: Normalized translational energy of scattered $\mathrm{H}$ atoms $E_{\mathrm{s}} / E_{\mathrm{i}}$. Polar axis: Scattering angle. Color coding: $\mathrm{H}$ atom scattering probability. 29 
M. Kammler et al. performed AIMD simulations based on DFT in order to explain the experimentally observed $\mathrm{H}$ atom scattering processes on graphene. 156 a This method gave rise to an adsorption barrier of $0.2 \mathrm{eV}$, a bond strength of $0.7 \mathrm{eV}$ with a $\mathrm{C}-\mathrm{H}$ bond length of $1.1 \AA$ and a carbon atom displacement of $0.4 \AA$.

Calculated translational energy losses of $\mathrm{H}$ atoms scattered from free-standing graphene with respect to the minimum $\mathrm{C}-\mathrm{H}$ scattering distance $d_{\min }$ are shown in Fig. 6.3 .

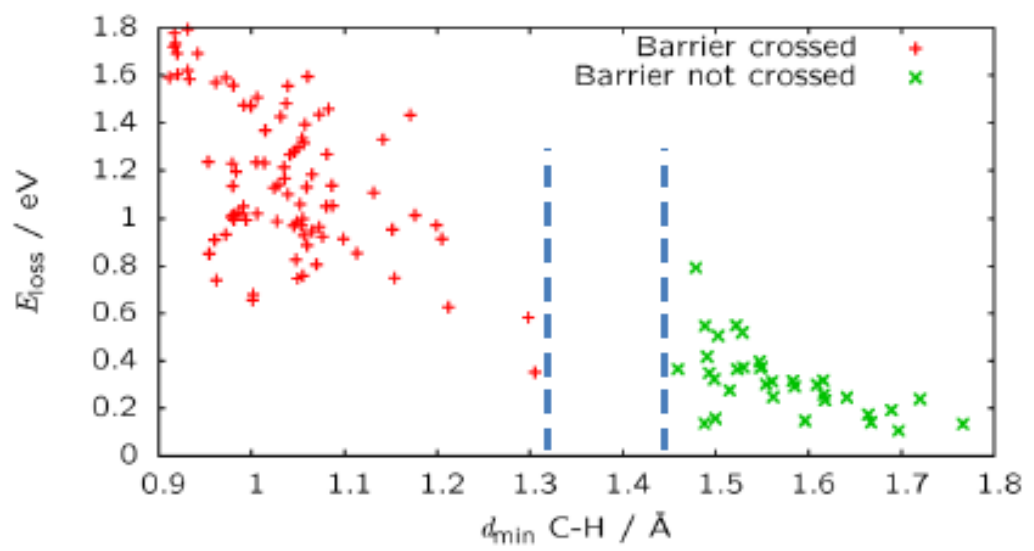

Figure 6.3: AIMD simulation of minimum $\mathrm{C}-\mathrm{H}$ atom distance during $\mathrm{H}$ atom scattering from free-standing graphene with respect to the energy loss of the scattered $\mathrm{H}$ atoms calculated at $E_{\mathrm{i}}=1.92 \mathrm{eV}$ and $\vartheta_{\mathrm{i}}=52^{\circ}$. The trajectories are separated in $\mathrm{H}$ atoms which cross (red pluses) and do not cross the adsorption barrier (green crosses). Blue dashed lines indicate the separation of the both scattering components. 29

Two types of trajectories were observed:

1. $d_{\min }>1.44 \AA$ : $\mathrm{H}$ atoms experience low energy losses (green crosses).

2. $d_{\text {min }}<1.33 \AA$ A $\mathrm{H}$ atoms experience significantly higher energy losses (red pluses).

The separation of both components is indicated by the blue dashed vertical lines. $\mathrm{H}$ atoms which strike the surface and cross the adsorption barrier experience a repulsive wall at shorter $\mathrm{C}-\mathrm{H}$ distances indicating the formation of a transient $\mathrm{C}-\mathrm{H}$ bond.

Figure 6.4 compares the 2D $\mathrm{H}$ atom translational energy loss and scattering angle distribution derived from AIMD calculations (upper panels, a and b) and experimental

${ }^{\text {a }}$ The simulations were carried out with VASP 5.3.5 (Vienna ab initio simulation package) using projectoraugmented waves in combination with pseudopotentials. ${ }^{157}[163$ Chosen slab size: 3 and 4 graphene unit cells in x- and y-direction, respectively; Energy cutoff: $400 \mathrm{eV}$; Step-size: $0.1 \mathrm{fs}$. The tetrahedron method with Blöchl corrections (smearing: $0.2 \mathrm{eV}$ and a $8 \times 8 \times 1$ k-point mesh centered at the $\Gamma$-point) were used in order to tread partial occupancies. Spin-polarization, the PBE functional and the D2 van der Waals correction were included in all calculations. Global break condition when total free energy and band structure energy change $<0.01 \mathrm{meV}$. A trajectory simulation was stopped if either the $\mathrm{H}$ atom reached its initial $\mathrm{H}$-surface distance or if the simulation time exceeded $200 \mathrm{fs}$. 

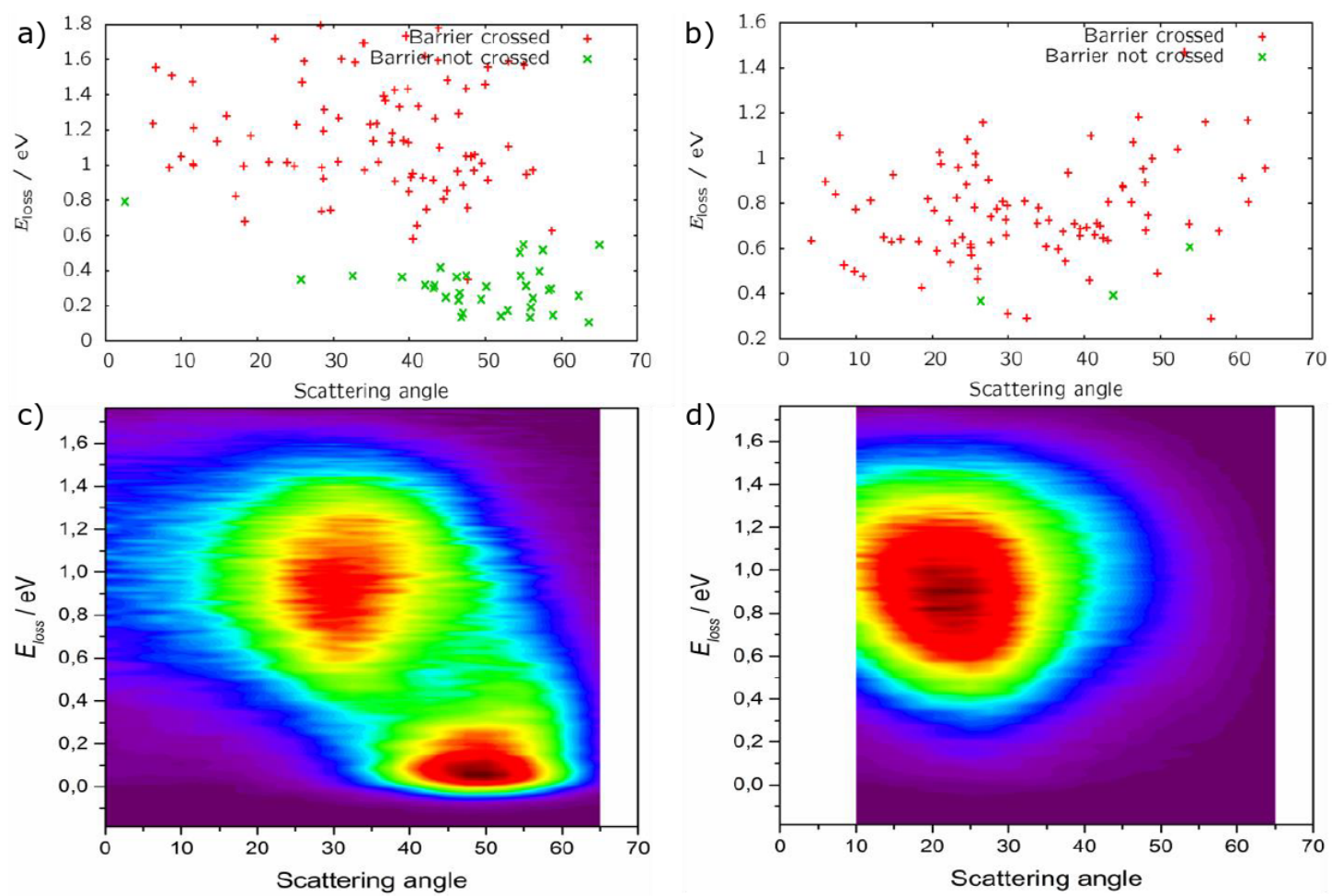

Figure 6.4: Upper panels: AIMD simulated tranjectory outcome of $\mathrm{H}$ atoms scattering from free-standing graphene with respect to the translational energy loss and scattering angle at $\vartheta_{\mathrm{i}}=52^{\circ}$ (a) and $\vartheta_{\mathrm{i}}=34^{\circ}$ (b) calculated by 122 and 86 tranjectories, respectively. The trajectories are separated in $\mathrm{H}$ atoms which cross (red pluses) and do not cross the adsorption barrier (green crosses). Lower panels: Experimentally derived $2 \mathrm{D} \mathrm{H}$ atom translational energy loss and scattering angle distributions measured at $\vartheta_{\mathrm{i}}=48^{\circ}(\mathrm{c})$ and $\vartheta_{\mathrm{i}}=30^{\circ}(\mathrm{d})$, respectively. Applied incidence energy: $E_{\mathrm{i}}=1.92 \mathrm{eV} .29$ 
measurements (lower panels, c and d) at two different incidence angles (left $\vartheta_{\mathrm{i}}=52^{\circ}$, right $\left.\vartheta_{\mathrm{i}}=34^{\circ}\right)$, respectively. Each point in the simulated AIMD spectra represents the outcome of one trajectory with respect to the scattering angle and final kinetic energy. The data points are separated into trajectories which cross the adsorption barrier (red pluses) and do not cross the adsorption barrier (green crosses). A qualitatively good overlap of the theoretical and experimentally determined data $\mathrm{a} / \mathrm{c}$ or b/d could been achieved when the data were compared at incidence angles shifted by approximately $4^{\circ}$. Thus, here the experimental data are presented at $\vartheta_{\mathrm{i}}=48^{\circ}$ (left, c) and $\vartheta_{\mathrm{i}}=30^{\circ}$ (right, d). The need to shift the incidence angle might be caused by inaccuracies in the theoretical calculations, as DFT is well know to underestimate the adsorption barrier height.

AIMD simulations and experimental measurements show at $\vartheta_{\mathrm{i}}=52^{\circ}$ (exp: $48^{\circ}$ ) (left panels) two components: A fast component with low energy loss and narrow angular distribution and a slow component with a broader energy loss and angular distribution. These components were assigned by theoretical calculations to $\mathrm{H}$ atom trajectories which do not cross the adsorption barrier and to $\mathrm{H}$ atom trajectories which involve barrier crossing.

At higher normal incidence energies (low incidence angles, right panels) only the slow component can be observed which indicates that nearly all the $\mathrm{H}$ atoms cross the adsorption barrier. Jiang determined an adsorption threshold for $\mathrm{H}$ atoms impinging on the graphene surface in the range of $0.41-0.48 \mathrm{eV}$ by monitoring changes in the peak-to-peak ratio of the slow to the fast component. The measurements were performed in UHV at a relatively low $\mathrm{H}$ atom flux, thus, it can be concluded that the threshold was determined at basically zero-surface coverage.

The fast component is assigned to quasi-elastic scattering of $\mathrm{H}$ atoms on the graphene surface. Theoretical calculations predict for $\mathrm{H}$ atoms crossing the adsorption barrier a large and rapid energy loss due to the formation of a transient reaction complex. The interaction time of the $\mathrm{H}$ atoms and graphene surface is in the order of a few tens of femtoseconds. The large energy loss of the $\mathrm{H}$ atoms colliding with the graphene surface stems from the deformation of the graphene layer during the interaction process. Figure 6.5 illustrates the equilibrium configuration of a chemisorbed hydrogen atom on graphene. Not only the carbon atom which bonds to the hydrogen atom moves out of the graphene plane towards the hydrogen atom but also the neighboring atoms are significantly displaced from their initial position which implies an excitation of the bonding carbon atom and the neighboring carbon atoms. $153 \mid 164$

The AIMD simulations by Kammler et al. propose that the energy of the hydrogen atom is simultaneously transferred to several carbon atoms of the graphene layer as the 


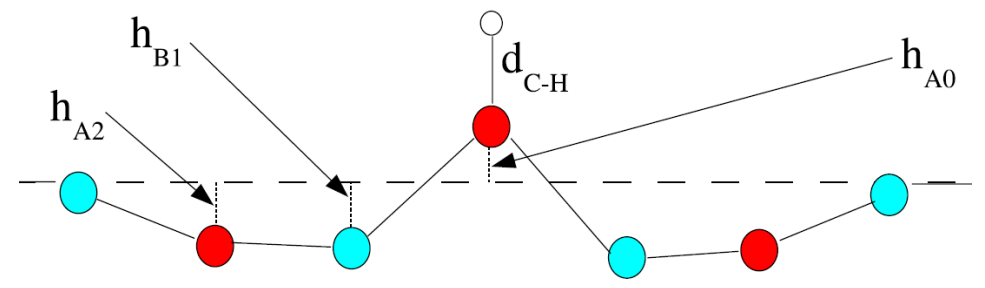

Figure 6.5: Simulated local distortion of the graphene layer at chemisorption of one hydrogen atom on graphene. The white circle shows the $\mathrm{H}$ atom and the red and cyan circles represent carbon atoms from two sub-lattices. The original graphene plane is indicated by the dashed line. 164

energy can dissipate over many degrees of freedom of the graphene layer. The simulations determined 69 normal modes with an energy range of $\nu_{\mathrm{vib}}=60-1583 \mathrm{~cm}^{-1}$. During the interaction time ( $t=38.9-51 \mathrm{fs})$ a substantial number of phonon modes can be excited at the same time which causes the large and rapid energy loss of the colliding hydrogen atom. Thus, the theoretical calculations indicate that the energy transfer between the hydrogen atom and graphene on $\mathrm{Pt}(111)$ is mainly adiabatic. Non-adiabatic excitation mechanism seem to play no or only a minor role on the energy transfer process between the incoming hydrogen atom and the graphene surface. 


\section{2 $\mathrm{H}$ atom scattering from Graphene on $\mathrm{Ni}(111)$}

In this section, experimental results of hydrogen atoms scattering from two different graphene monolayers on $\mathrm{Ni}(111)$ are presented: (1 x 1) oriented graphene and partially rotated (rot.) graphene. Translational energy and angular distributions of scattered hydrogen atoms from this graphene layers were measured at energies of $E_{\mathrm{i}}=0.51-1.92 \mathrm{eV}$ with respect to the incidence angle $\left(\vartheta_{\mathrm{i}}=30^{\circ}-60^{\circ}\right.$ in $2^{\circ}$ steps $)$ and scattering angle $\left(\vartheta_{\mathrm{s}} \approx 0^{\circ}-75^{\circ}\right.$ in $2.5^{\circ}$ steps $)$ at $T_{\mathrm{s}}=300 \mathrm{~K}$.

In the first section, experimental data obtained from scattering experiments from graphene on $\mathrm{Ni}(111)$ are compared and discussed to scattering results from the pristine nickel substrate surface (previously discussed in Chapter 4)). For the discussion only one graphene on $\mathrm{Ni}(111)$ modification will be compared, as both modifications exhibit similar characteristic features. However, a closer comparison of $(1 \times 1)$ oriented graphene and rotated graphene on $\mathrm{Ni}(111)$ revealed significant differences between both modifications which will be consecutively discussed and compared to $\mathrm{H}$ atom scattering results from $\mathrm{Gr} / \mathrm{Pt}(111)$.

\subsubsection{Comparison between $(1 \times 1)$ Graphene on $\mathrm{Ni}(111)$ and pristine $\mathrm{Ni}(111)$}

Angular distributions of $\mathrm{H}$ atoms scattering from $(1 \times 1)$ graphene on $\mathrm{Ni}(111)$ and the clean $\mathrm{Ni}(111)$ substrate are presented in Figure 6.6. Drastic differences in the angular distributions are observed for both samples. In case of clean $\mathrm{Ni}(111)$ surface, the angular distributions are broad and have an almost cosine shape which is nearly independent of the incidence energy and angle. In contrast, results from graphene on $\mathrm{Ni}(111)$ show angular distributions which are effected by the incidence energy and angle conditions. Here, angular distributions at two different incidence energies are presented: $E_{\mathrm{i}}=1.92 \mathrm{eV}$ (blue squares) and $E_{\mathrm{i}}=0.99 \mathrm{eV}$ (red triangles). $\mathrm{H}$ atoms scattered from graphene at $E_{\mathrm{i}}=0.99 \mathrm{eV}$ exhibit a relatively narrow angular distribution $\left(\mathrm{FWHM} \approx 25^{\circ}\right)$ and peak at the specular angle (here $\vartheta_{\mathrm{s}}=40^{\circ}$ ). At an incidence energy of $1.92 \mathrm{eV}$, a broadening of the angular distribution $\left(\mathrm{FWHM} \approx 35^{\circ}\right)$ was observed with a peak shifted closer to the surface normal $\left(\vartheta_{\mathrm{s}} \approx 30^{\circ}\right)$. 


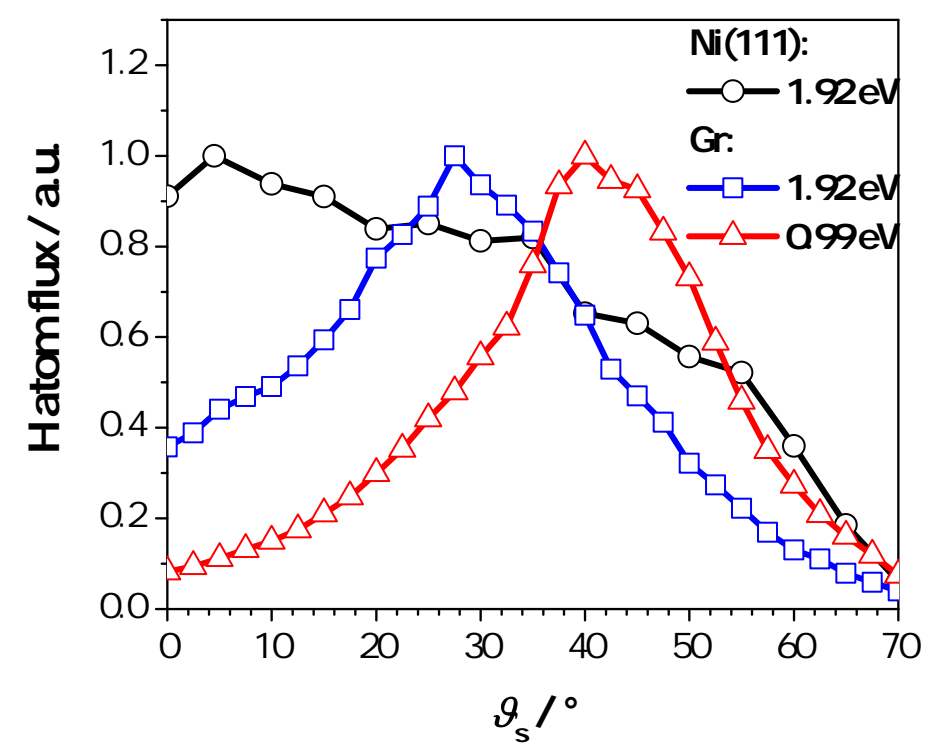

Figure 6.6: Angular distributions of $\mathrm{H}$ atoms scattered from $(1 \times 1)$ graphene on $\mathrm{Ni}(111)$ at $E_{\mathrm{i}}=1.92 \mathrm{eV}$ (blue squares) and $E_{\mathrm{i}}=0.99 \mathrm{eV}$ (red triangles) and pristine $\mathrm{Ni}(111)$ surface (black circles) at $E_{\mathrm{i}}=1.92 \mathrm{eV}$. All angular distributions were measured at an incidence angle of $\vartheta_{\mathrm{i}}=40^{\circ}$. The lines are used to guide the eye.

Figure 6.7 presents translational energy distributions of $\mathrm{H}$ atoms scattered from $(1 \mathrm{x} 1)$ graphene on $\mathrm{Ni}(111)$ (squares) and the pristine $\mathrm{Ni}(111)$ surface (circles) measured at $\vartheta \mathrm{i}=\vartheta_{\mathrm{s}}=45^{\circ}$ and two different incidence energies $\left(E_{\mathrm{i}}=1.92 \mathrm{eV}\right.$ : filled symbols; $E_{\mathrm{i}}=0.99 \mathrm{eV}$ : open symbols).

$\mathrm{H}$ atoms scattering from a pristine nickel surface experience a large average energy loss with a broad and structureless energy distribution which shape is nearly unaffected by incidence energy and incidence or scattering angle.

In contrast, energy distributions for $\mathrm{H}$ atoms scattering from graphene on $\mathrm{Ni}(111)$ drastically change with varying incidence energy and angle. Energy distributions at the same incidence and scattering angle but different incidence energies are shown. A broad energy distribution is observed at high incidence energies, while at low incidence energy a bimodular shape with reduced average energy loss is observed.

No indications for scattering from the metal substrate have been observed in the measured graphene spectra. This provides evidence that a complete graphene layer was built on the $\mathrm{Ni}(111)$ surface and that only hydrogen atoms are detected which scatter directly from the graphene layer. 


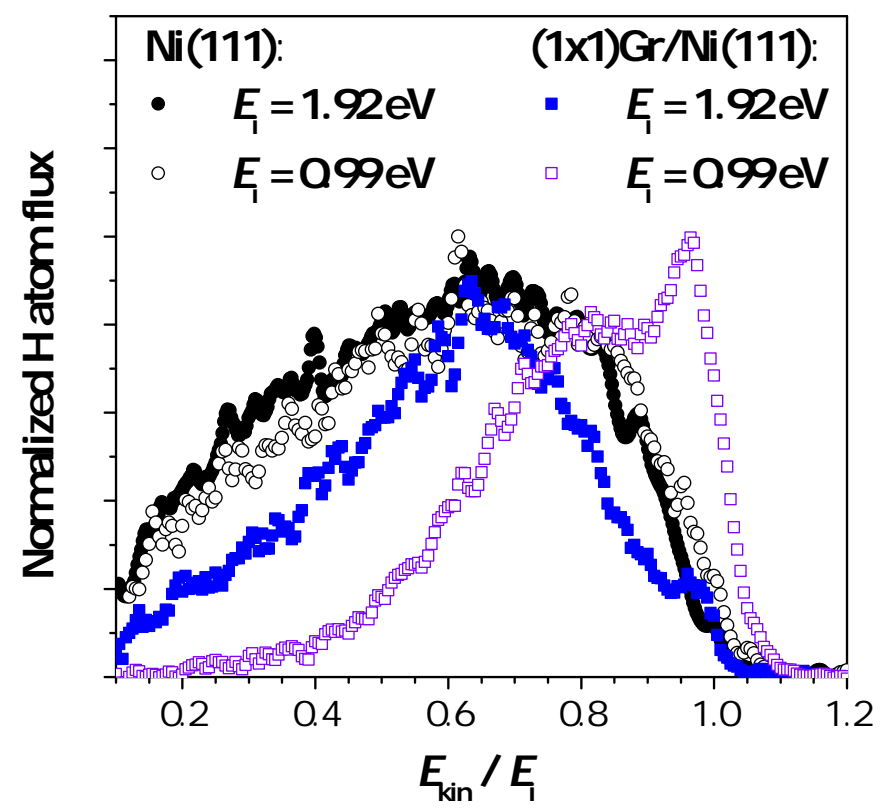

Figure 6.7: Translational energy distributions of $\mathrm{H}$ atoms scattered from a $(1 \mathrm{x} 1)$ graphene on $\mathrm{Ni}(111)$ (filled blue and open purple squares) and pristine $\mathrm{Ni}(111)$ surface (filled black and open circles) at $\vartheta \mathrm{i}=\vartheta_{\mathrm{s}}=45^{\circ}$. Incidence energy $E_{\mathrm{i}}=0.99 \mathrm{eV}$ for open symbols and $1.92 \mathrm{eV}$ for filled symbols.

\subsubsection{D Transaltional energy and angular distribution spectra of Graphene on Ni(111)}

A serious of energy distributions of $\mathrm{H}$ atoms scattered from $(1 \times 1)$ oriented and rotated $\mathrm{Gr} / \mathrm{Ni}(111)$ were measured at various incidence energies and angles with respect to the scattering angle.

2D translational energy and scattering angle distributions of hydrogen atoms scattered from rot. $\mathrm{Gr} / \mathrm{Ni}(111)$ at different incidence angles $\vartheta_{\mathrm{i}}$ (top left $\rightarrow$ bottom right: decreasing incidence angle) at $E_{\mathrm{i}}=1.92 \mathrm{eV}$ are presented in Fig. 6.8. The color codings represent the relative scattering probability for each experimental condition.

At low normal incidence energies (top left), $\mathrm{H}$ atoms mainly scatter from the graphene surfaces around the specular scattering angle with a narrow angular and energy distribution (FWHM: $15^{\circ}$ and $100 \mathrm{meV}$ ). The signal peaks at approximately $0.98 \cdot E_{\mathrm{i}}\left(E_{\text {loss }} \approx 10 \mathrm{meV}\right)$ and decreases with increasing normal incidence energy, similar to the quasi-elastic fast scattering component observed from $\mathrm{H}$ atom scattering from graphene on $\mathrm{Pt}(111)$, al- 

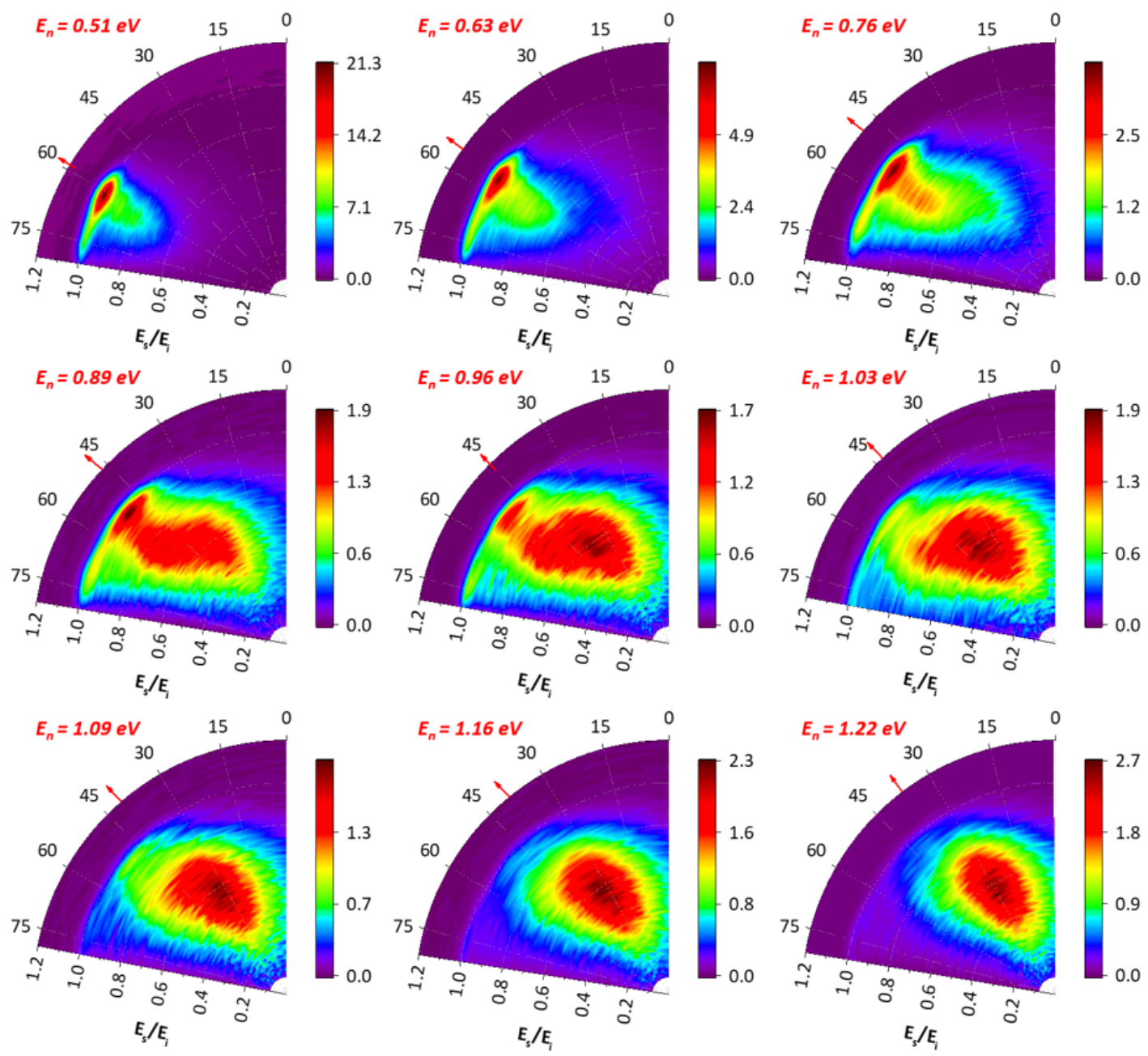

Figure 6.8: $2 \mathrm{D}$ translational energy and scattering angular distributions of $\mathrm{H}$ atoms scattered from rot. $\mathrm{Gr} / \mathrm{Ni}(111)$ measured at fixed incidence energy $E_{\mathrm{i}}=1.92 \mathrm{eV}$ but different incidence angles. The red arrows mark the specular scattering angle. The figures are labeled with the corresponding normal incidence energy. 

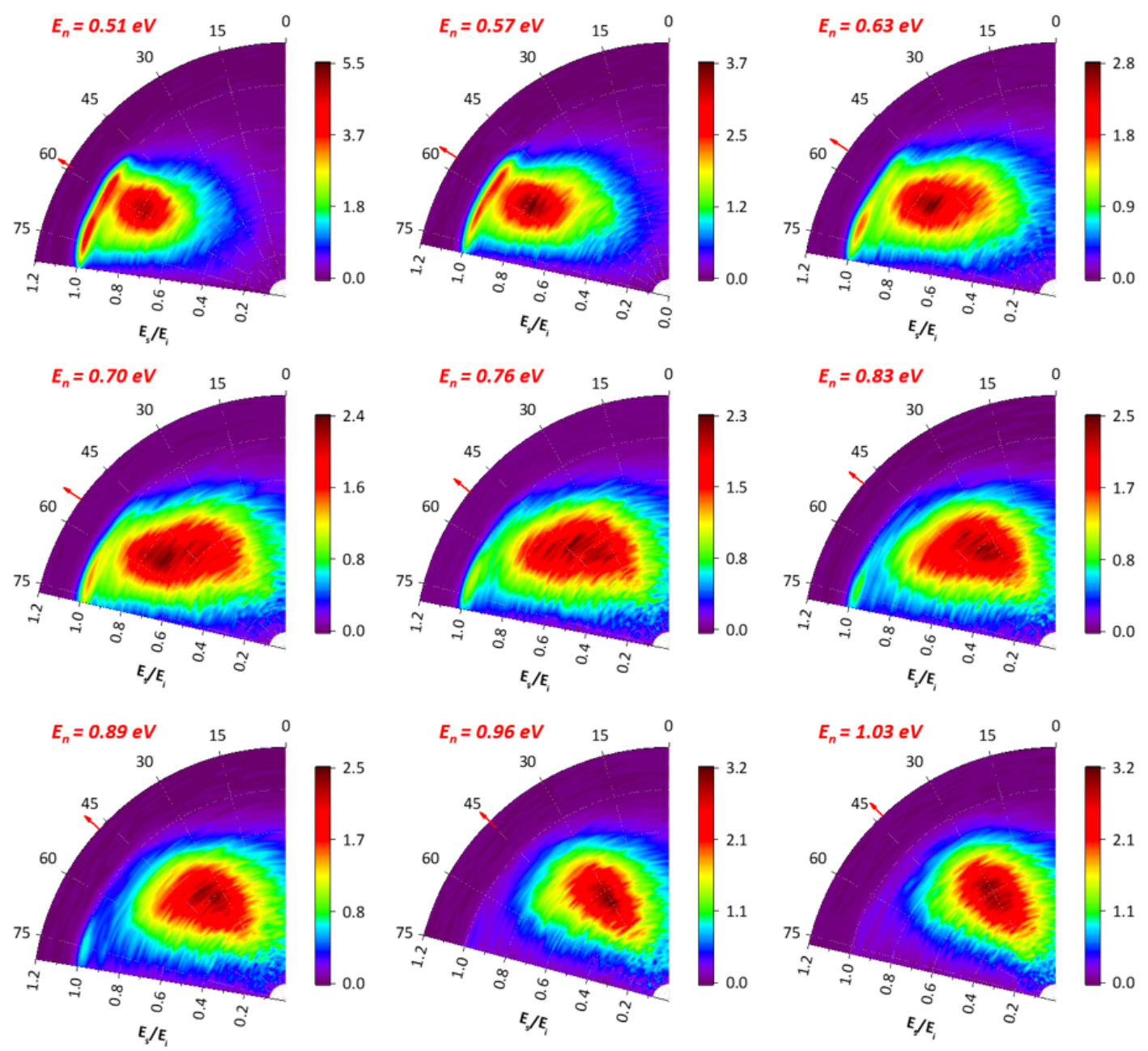

Figure 6.9: 2D translational energy and scattering angular distributions of $\mathrm{H}$ atoms scattered from $(1 \times 1) \mathrm{Gr} / \mathrm{Ni}(111)$ measured at fixed incidence energy $E_{\mathrm{i}}=1.92 \mathrm{eV}$ but different incidence angles. The red arrows mark the specular scattering angle. The figures are labeled with the corresponding normal incidence energy. 
though the angular distribution of this fast component exhibits a small tail towards higher scattering angles.

Furthermore, an additional new feature with higher energy losses $\left(E_{\mathrm{s}, \max } / E_{\mathrm{i}} \approx 75 \%\right)$ can be observed, which has an angular distribution similar to the quasi-elastic peak (FWHM: $15^{\circ}$, peak maximum around the specular scattering angle). This new component decreases as well with increasing normal incidence energy and vanishes for normal incidence energies greater than $1.03 \mathrm{eV}$.

At $E_{\mathrm{n}}=0.63 \mathrm{eV}$, another component with higher energy losses $\left(E_{\mathrm{s}} / E_{\mathrm{i}} \approx 50 \%\right)$ and broader angular distribution (FWHM: $30^{\circ}$ ) arises, which increases with the normal incidence energy. This slow component peaks closer to the surface normal around $\vartheta_{\mathrm{s}}=30^{\circ}$. The scattering dynamics of the component are similar to the slow component observed from $\mathrm{H}$ atoms scattered from graphene on $\mathrm{Pt}(111)$.

2D translational energy and scattering angle distributions of hydrogen atoms scattered from $(1 \times 1) \mathrm{Gr} / \mathrm{Ni}(111)$ (Fig. 6.9) show similar features as rot. $\mathrm{Gr} / \mathrm{Ni}(111)$ but different ratios with respect to all scattering components. Both the fast component $\left(E_{\mathrm{s}, \max }=0.98 \cdot E_{\mathrm{i}}\right)$ and the new component $\left(E_{\mathrm{s}, \max } \approx 0.75 \cdot E_{\mathrm{i}}\right)$ vanish at lower normal incidence energies (approx. $E_{\mathrm{n}}=0.83 \mathrm{eV}$ ), while the slow component $\left(E_{\mathrm{s}, \max } \approx 0.5 \cdot E_{\mathrm{i}}\right)$ starts to arise at $E_{\mathrm{n}}=0.57 \mathrm{eV}$.

In addition, a distinct broadening of the angular distribution of the fast component $\left(E_{\text {kin,max }} \approx 0.98 \cdot E_{\mathrm{i}}\right)$ towards higher scattering angles than the specular scattering angle can be observed.

Additional 2D translational energy and angular distributions of hydrogen atoms scattered from rot. $\mathrm{Gr} / \mathrm{Ni}(111)$ and $(1 \times 1) \mathrm{Gr} / \mathrm{Ni}(111)$ at lower incidence energies $\left(E_{\mathrm{i}}=0.99 \mathrm{eV}\right.$ and $0.51 \mathrm{eV}$ ) can be found in Appendix C. 


\subsection{Quasi-elastic scattering component}

Upon closer examination and subsequent modeling of the fast peak $\left(E_{\mathrm{kin}, \max } \approx 0.98 \cdot E_{\mathrm{i}}\right)$, it was found out that at high incidence energies $\left(E_{\mathrm{i}}=1.92 \mathrm{eV}\right)$ this component actually consists of at least two subcomponents (see Fig. 6.10). This is more obvious for $(1 \times 1)$

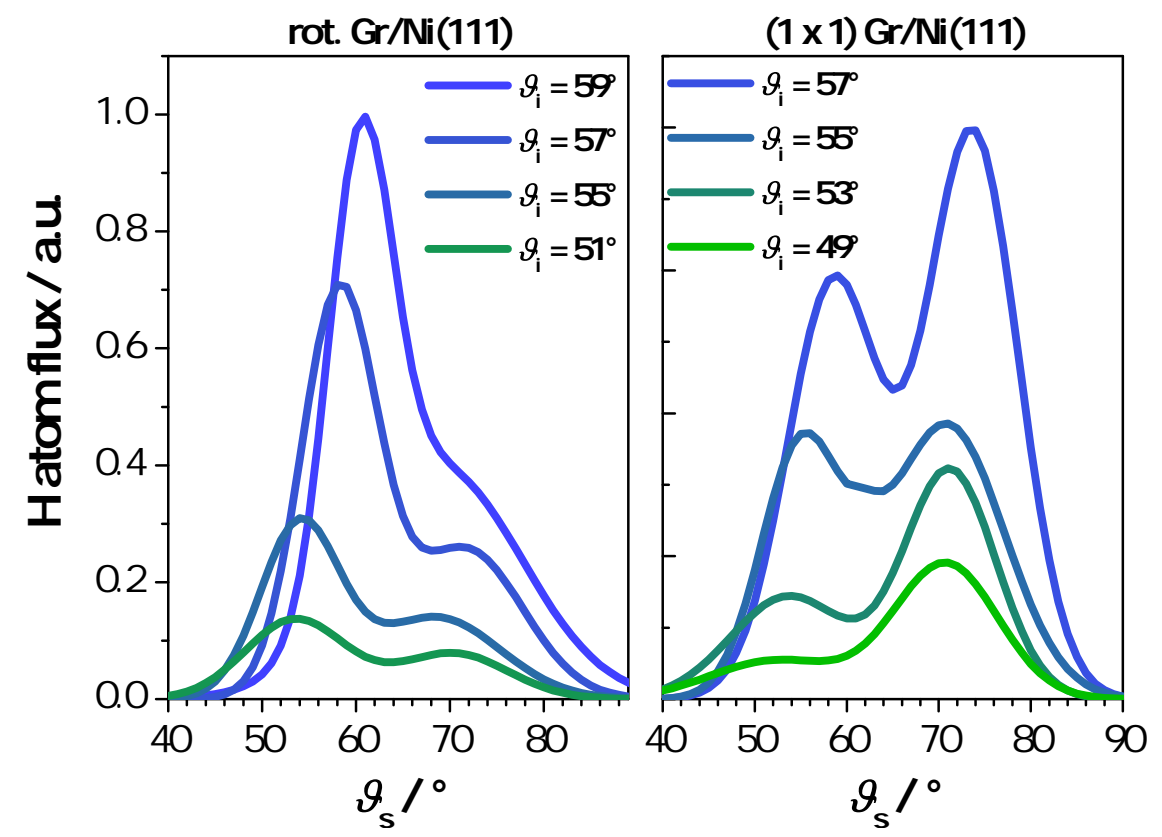

Figure 6.10: Fitted angular distributions of the quasi-elastic peak component of $\mathrm{H}$ atoms scattered from rot. $\mathrm{Gr} / \mathrm{Ni}(111)$ (left panel) and (1 x 1) Gr/Ni(111) (right panel) measured at various incidence angles at $E_{\mathrm{i}}=1.92 \mathrm{eV}$.

$\mathrm{Gr} / \mathrm{Ni}(111)$, while for rot. $\mathrm{Gr} / \mathrm{Ni}(111)$ one component seems to strongly dominate. This effect for $(1 \times 1) \mathrm{Gr} / \mathrm{Ni}(111)$ might be explained by two geometrically inequivalent carbon atoms (top vs. $f c c$-hollow sides, see Chapter 2.2.3 resulting from the strong graphenemetal interaction. Whether the second peak for rot. $\mathrm{Gr} / \mathrm{Ni}(111)$ results either from a similar effect or corresponds only to the minor fraction of (1 x 1) $\mathrm{Gr} / \mathrm{Ni}(111)$ inherent in the rot. $\mathrm{Gr} / \mathrm{Ni}(111)$ sample cannot be determined as their relative abundancies are unknown for the investigated samples. Thus, theoretical calculations are required in order to fully explain these observations. These subcomponents will therefore not be further discussed in this thesis. 


\subsection{Comparison between Graphene on $\mathrm{Ni}(111)$ and Graphene on $\operatorname{Pt}(111)$}

Certain similarities between $\mathrm{H}$ atom scattering from $\mathrm{Gr} / \mathrm{Ni}(111)$ and $\mathrm{Gr} / \mathrm{Pt}(111)$ were observed. Figure 6.11 depicts translational energy distributions of $\mathrm{H}$ atoms scattered from $\mathrm{Gr} / \mathrm{Pt}(111)$ (left panel) and rot. $\mathrm{Gr} / \mathrm{Ni}(111)$ (right panel) at $\vartheta_{\mathrm{i}}=60^{\circ}$ and $E_{\mathrm{i}}=1.92 \mathrm{eV}$ measured at various scattering angles.

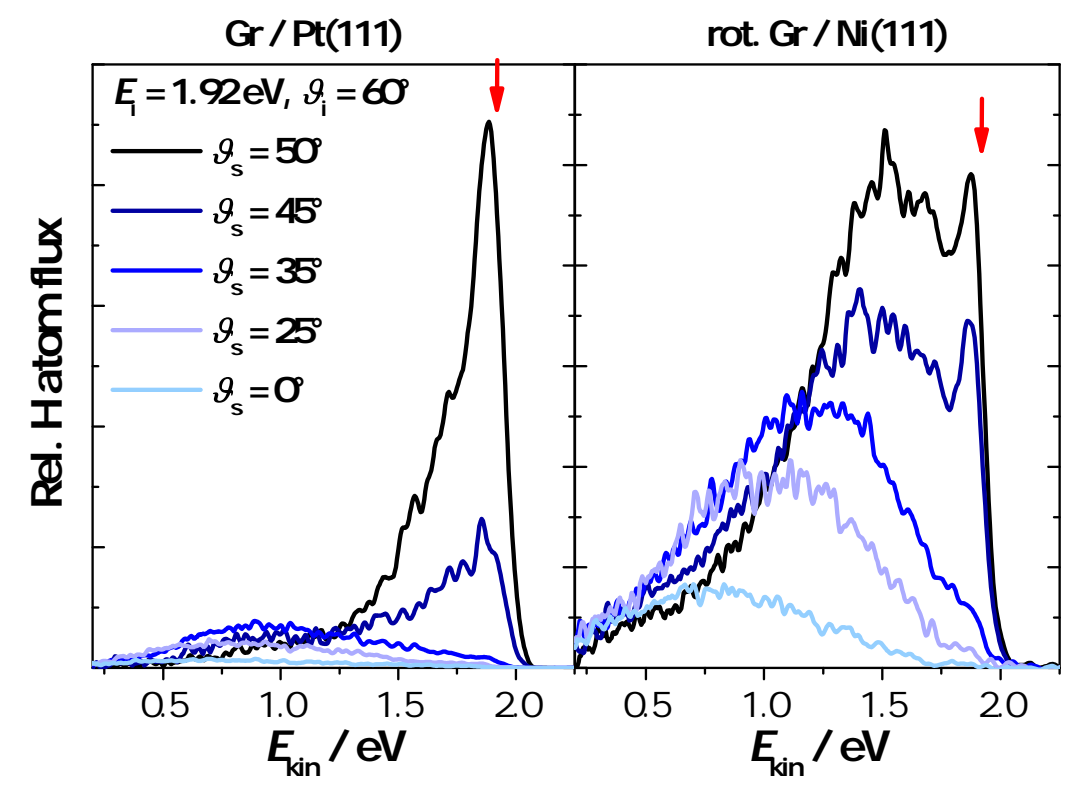

Figure 6.11: Comparison between translational energy distributions of $\mathrm{H}$ atoms scattered from $\mathrm{Gr} / \mathrm{Pt}(111)$ (left panel) and rot. $\mathrm{Gr} / \mathrm{Ni}(111)$ (right panel) at $\vartheta_{\mathrm{i}}=60^{\circ}$ and $E_{\mathrm{i}}=1.92 \mathrm{eV}$ measured at representative scattering angles (labeled in the left panel). The vertical red arrows mark the incidence energy.

Both graphene surfaces exhibit a sharp hydrogen atom scattering peak with low energy loss $E_{\text {kin,max }} / E_{\mathrm{i}} \approx 98 \%$. This narrow component decreases with decreasing scattering angle, indicative of similar scattering dynamics for both samples. Comparison of theoretical simulations with $\mathrm{H}$ atom scattering experiments on $\mathrm{Gr} / \mathrm{Pt}(111)$ previously have revealed that this component results from a quasi-elastic scattering on the graphene surface without barrier crossing (Scenario A).

At scattering angles close to the surface normal, in case of $\mathrm{Gr} / \mathrm{Pt}(111)$, a new feature starts to arise. This feature shows a much higher average energy loss of approximately $50 \%$ of the incidence energy and could be identified as hydrogen atoms scattered from graphene after adsorption barrier crossing (Scenario B). 
In contrast, for $\mathrm{Gr} / \mathrm{Ni}(111)$ the spectra suggest more complex scattering dynamics. While at low scattering angles similar spectra compared to $\mathrm{Gr} / \mathrm{Pt}(111)$ were obtained $\left(\left\langle E_{\text {kin }}\right\rangle / E_{\mathrm{i}} \approx 50 \%\right)$, with increasing scattering angle the energy distribution first exhibits a plateau before the peak maximum shifts towards higher kinetic energies $\left(\left\langle E_{\mathrm{kin}}\right\rangle / E_{\mathrm{i}} \approx 75 \%\right)$. This observation suggests the presence of two separate scattering processes. On one hand, the slow component predominant at low scattering angles can most likely be assigned to $\mathrm{H}$ atom scattering from graphene via Scenario B as for $\mathrm{Gr} / \mathrm{Pt}(111)$. On the other hand, the new third component has previously not been observed on $\mathrm{Gr} / \mathrm{Pt}(111)$ and requires further investigations. 


\subsection{Comparison between $(1 \times 1)$ oriented and rotated Graphene on $\mathrm{Ni}(111)$}

After the scattering processes have been discussed for rot. $\mathrm{Gr} / \mathrm{Ni}(111)$, in the following section these results will be compared to the alternative $(1 \times 1)$ orientated graphene modification. In Figure 6.12 an overview of total energy distributions (summed over all scattering angles) measured at various incidence angles and at two different incidence energies for both modifications can be found.

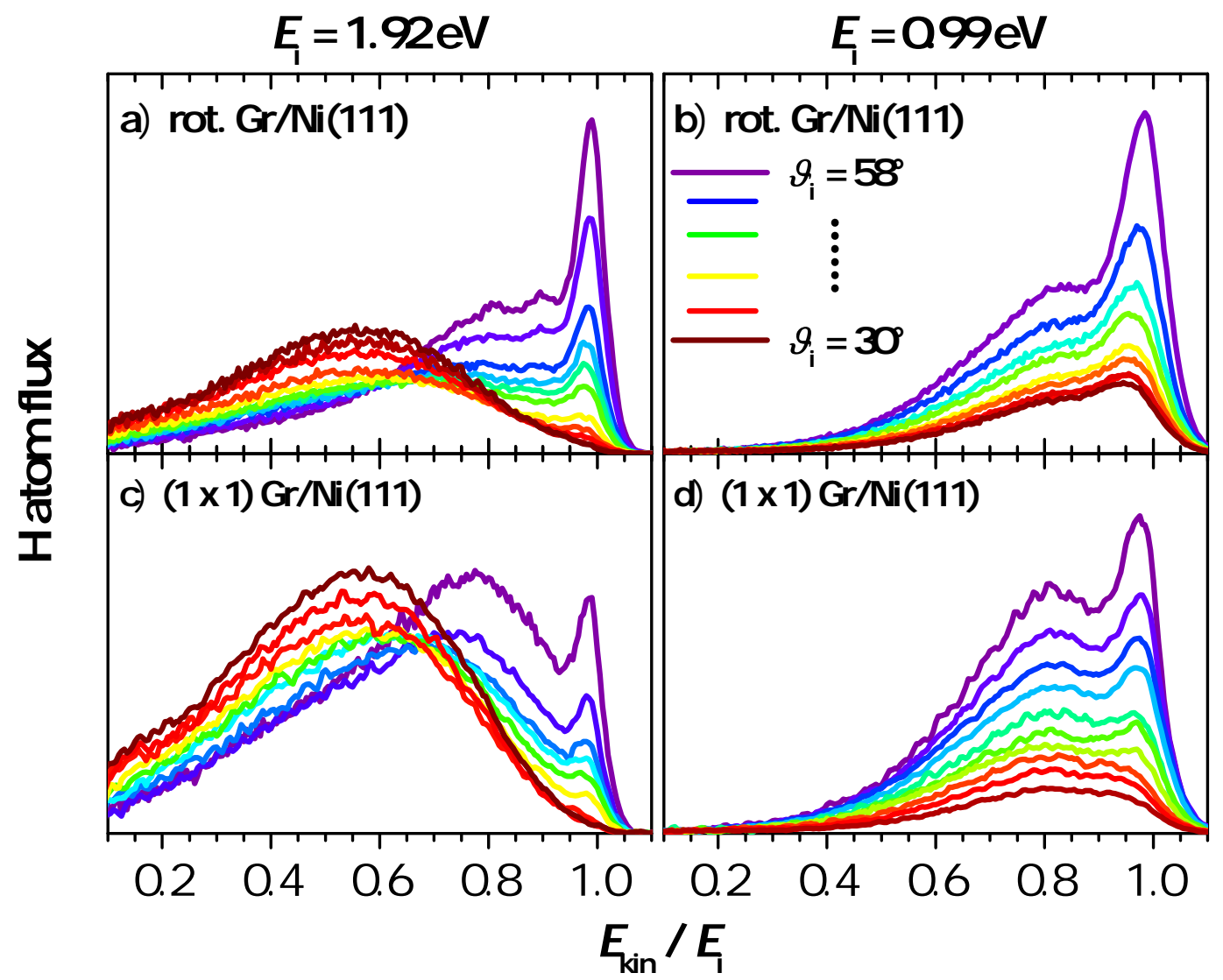

Figure 6.12: Total translational energy distributions of $\mathrm{H}$ atoms scattered from rot. $\mathrm{Gr} / \mathrm{Ni}(111)$ (upper panels) and $(1 \times 1) \mathrm{Gr} / \mathrm{Ni}(111)$ (lower panels) at $E_{\mathrm{i}}=1.92 \mathrm{eV}$ (a and c) and $0.99 \mathrm{eV}$ ( $\mathrm{b}$ and $\mathrm{d}$ ). The different colored lines correspond to energy distributions derived from measurements at different incidence angles (color coding for are panels is listed in panel b).

This comparison reveals that all components that have been previously established for rot. $\mathrm{Gr} / \mathrm{Ni}(111)$ could be found for $(1 \times 1) \mathrm{Gr} / \mathrm{Ni}(111)$ as well, however, their relative 
contributions varies for both modifications.

For instance, when comparing a) and c) it becomes clearly evident that the quasi-elastic peak (Scenario A, $E_{\text {kin,max }} / E_{\mathrm{i}} \approx 2 \%$ ) decreases relatively to the two other components for $(1 \times 1) \mathrm{Gr} / \mathrm{Ni}(111)$ (c), which indicates a smaller reaction barrier for this modification. For lower incidence energies (b and d), the slow component $\left(E_{\mathrm{kin}, \max } / E_{\mathrm{i}} \approx 50 \%\right)$, corresponding to adsorption barrier crossing, is no longer observed, which might be explained by a higher adsorption probability for impinging hydrogen atoms for lower incidence energies. Further, a relative increase for the new third component $\left(E_{\text {kin,max }} / E_{\mathrm{i}} \approx 25 \%\right)$ compared to the quasi-elastic fast peak can be observed for increasing normal incidence energies and for $(1 \times 1) \mathrm{Gr} / \mathrm{Ni}(111)$ (b vs d). However, the scattering probability decreases for both components with decreasing incidence angle (corresponding to increasing $E_{\mathrm{n}}$ ) suggesting that both scattering processes do not involve adsorption barrier crossing.

It can be concluded that the same scattering processes are present for both graphene modifications, however, their inherently different properties clearly effect the efficiency of these processes. In order to gain a deeper understanding of the third component and its underlying dynamics, theoretical calculations are required. However, a possible explanation for the observed effects will be given in the discussion. 


\subsection{Scattering dynamics at different azimuthal orientations}

AIMD simulations performed by Kammler et al.156 and $\mathrm{H}$ atom diffraction calculations on graphite (0001) 19 predicted that most of the impinging $\mathrm{H}$ atoms on graphene are directed towards the C-C bond of the graphene surface. Thus, substantial differences should be observed in the scattering probability when changing the surface orientation which can be easily performed for the well ordered (1 x 1) Gr/Ni(111).

The comparison of angular and energy distributions of $\mathrm{H}$ atoms scattered from $(1 \times 1)$ $\mathrm{Gr} / \mathrm{Ni}(111)$ along two different surface orientations show clear differences for the [112] direction of the $\mathrm{Ni}(111)$ surface (along the $\mathrm{C}-\mathrm{C}$ graphene bond) and the [101] direction of the $\mathrm{Ni}(111)$ surface (rotated $30^{\circ}$ away from the C-C graphene bond) at $E_{\mathrm{i}}=0.99 \mathrm{eV}$ (Fig. 6.13).

Although similar angular and energy distributions for both surface orientations are observed, significantly different scattering intensities were recorded for both surface orientations, especially at high incidence angles.

Scattering along the C-C graphene bond seemingly exhibits a substantial lower scattering probability compared to scattering directions perpendicular to the $\mathrm{C}-\mathrm{C}$ bond. In the first place, this observations seems to contradict the theoretical assumption. Nevertheless, AIMD calculations further predict that most of the $\mathrm{H}$ atoms that cross the adsorption barrier are not scattered in the scattering plane but instead out of the scattering plane. Thus, a higher scattering probability of the components without barrier crossing might be observed for orientations of the impinging $\mathrm{H}$ atom perpendicular to the $\mathrm{C}-\mathrm{C}$ bond as they less likely cross the adsorption barrier. For a more detailed analysis of these finding, out-of-plane measurements are required which was not possible with the current experimental set-up. Additionally, the azimuth dependent measurements were only performed at $E_{\mathrm{i}}=0.99 \mathrm{eV}$, thus, it might be worthwhile investigating if at higher incidence energies the component resulting from adsorption barrier crossing shows similar azimuthal dependencies. 

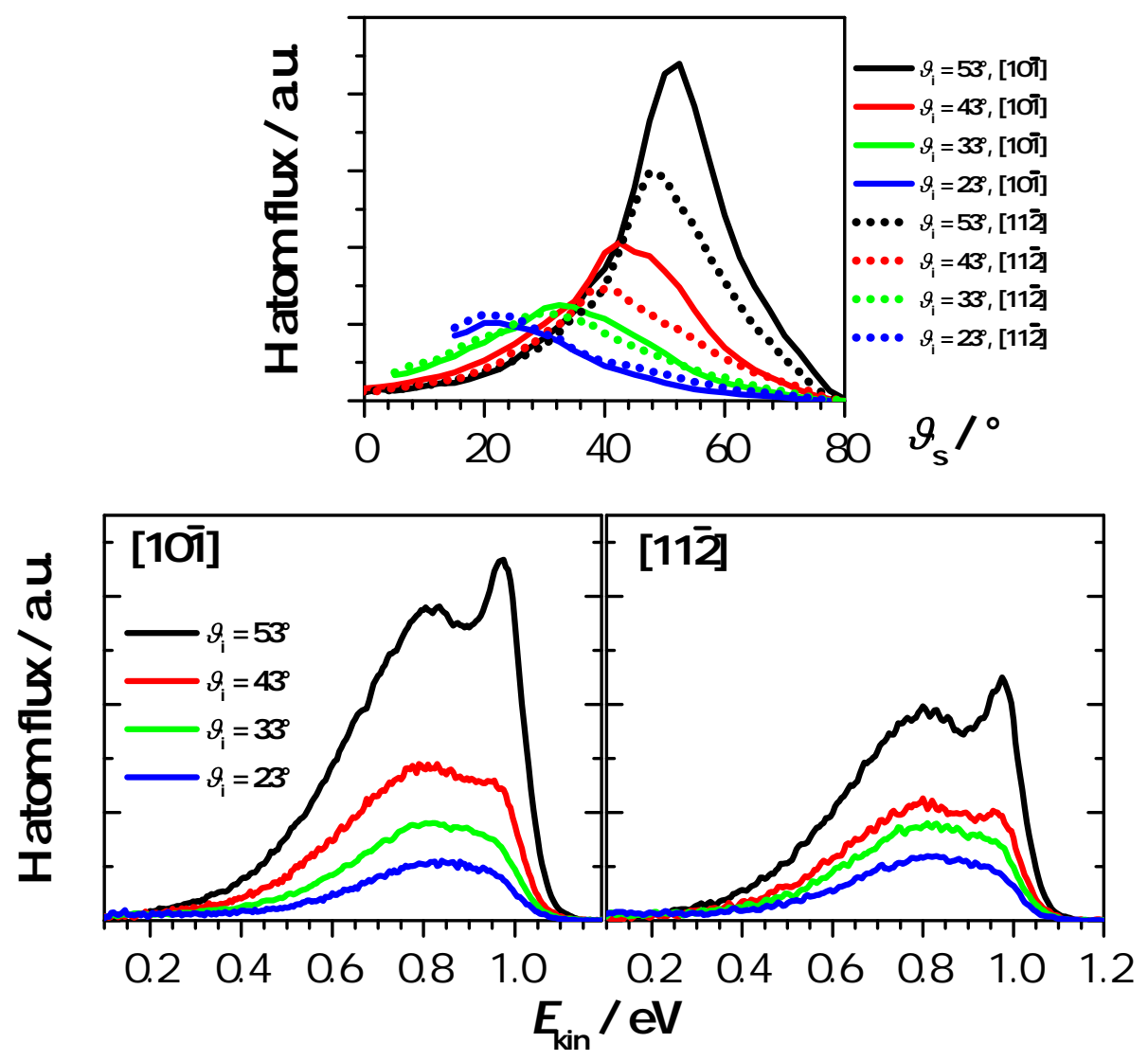

Figure 6.13: Upper panel: Angular distributions of $\mathrm{H}$ atom scattered from (1 x 1) $\mathrm{Gr} / \mathrm{Ni}(111)$ measured at various incidence angles and two different azimuthal orientations of the Ni(111) surface. Lower panels: Total translational energy distributions of $\mathrm{H}$ atoms scattered from $(1 \times 1) \mathrm{Gr} / \mathrm{Ni}(111)$ at different incidence angles (labeled in

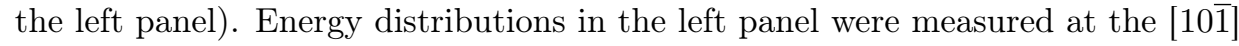
direction of the $\mathrm{Ni}(111)$ surface while in the right panel energy distributions were measured at the $[11 \overline{2}]$ direction of the $\mathrm{Ni}(111)$ surface. Experimental conditions: $E_{\mathrm{i}}=0.99 \mathrm{eV}$. 


\subsection{Adsorption threshold determination}

After the main characteristics for the spectra obtained for both graphene modifications have been discussed, the adsorption threshold for $\mathrm{H}$ atoms impinging on $\mathrm{Gr} / \mathrm{Ni}(111)$ will be derived. The $\mathrm{H}$ atom scattering experiments on the graphene surfaces were performed in UHV at a relatively low $\mathrm{H}$ atom beam flux $\left(10^{8}-10^{9}\right.$ atoms $\left./ \mathrm{cm}^{2} \mathrm{~s}\right)$ and thus the adsorption threshold will be determined at zero hydrogen atom coverage.

In order to determine the threshold, first all components had to be mathematically separated in order to obtain their relative scattering intensity ratios. The scattering intensities of the individual components were calculated by integration over the respective energy and angular distributions. In order to gain the full 3D peak intensities (including out-of-plane scattering), due to the lack of out-of-plane measurements, it was assumed that the angular distribution of all components is spherical for in-plane and out-of-plane scattering (for details see Appendix D). However, theoretical calculations indicate that this assumption may have to be corrected. Nevertheless, due to the lack of further information, the simplified initial approach was continued.

Generally, the best way to determine the adsorption threshold would be to plot the components resulting from scattering events without barrier crossing against the normal incidence energy. When these components reach saturation, it implies that the sticking probability of striking $\mathrm{H}$ atoms on the surface tends towards zero and no collision occurs with barrier crossing. During the performed experiments saturation of the quasi-elastic component could not be reached, thus, the adsorption thresholds were determined by an alternative method that has been previously applied for $\mathrm{Gr} / \mathrm{Pt}(111) .29$

This approach assumes that when the scattering intensity ratio $P_{\mathrm{bc}} / P_{\mathrm{nbc}}$ resulting from $\mathrm{H}$ atom scattering with barrier crossing $P_{\mathrm{bc}}$ and without barrier crossing $P_{\mathrm{nbc}}$ is larger than zero, there are some hydrogen atoms which have enough normal incidence energy to overcome the adsorption barrier. A mathematical relation of the scattering intensity ratio with respect to the normal incidence energy $E_{\mathrm{n}}$ was developed (see Eq. 6.2.)

$$
\frac{P_{\mathrm{bc}}}{P_{\mathrm{nbc}}}=k \cdot\left(1+\frac{t}{\left(E_{\mathrm{n}}-E_{0}\right)^{n}-t}\right), \quad E_{\mathrm{n}}>E_{0}
$$

with $k, t$ and $n$ being scaling factors, which depend on the incidence energy, surface temperature, sticking probability, etc. and $E_{0}$ corresponding to the adsorption threshold. The scattering intensity ratio of the components resulting from $\mathrm{H}$ atom scattering from $\mathrm{Gr} / \mathrm{Ni}(11)$ with barrier crossing and without barrier crossing with respect to the normal incidence energy are presented in Fig. 6.14 for both graphene modifications. 


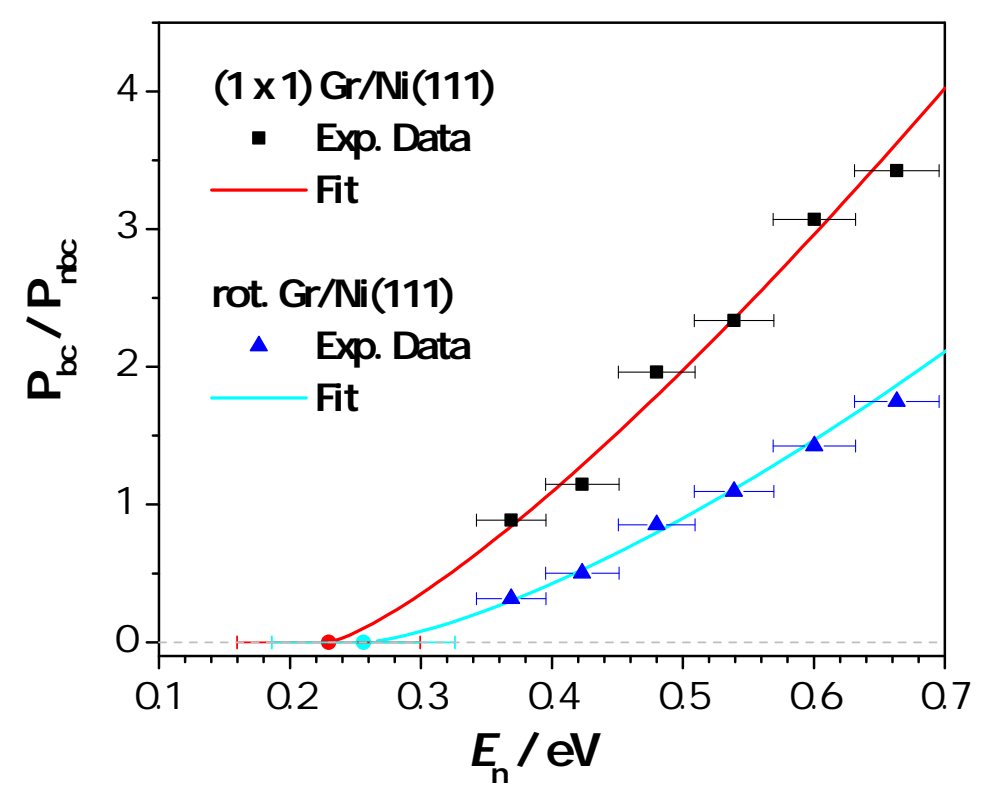

Figure 6.14: Intensity ratio of the slow peak to the fast peak for $\mathrm{H}$ atoms scattering from rot. $\mathrm{Gr} / \mathrm{Ni}(111)$ (open blue symbols) and (1 x 1) Gr/Ni(111) (filled black symbols) with respect to the normal incidence energies. The arrow bars in the normal energy result from the $\mathrm{H}$ atom beam incidence energy width $( \pm 10 \mathrm{meV})$ and incidence angle uncertainty $\left( \pm 0.1^{\circ}\right)$. The red and blue line show the corresponding best fit of the data points. 
Only $P_{\mathrm{bc}} / P_{\mathrm{nbc}}$ ratios could be determined for $E_{\mathrm{i}}=1.92 \mathrm{eV}$ as the component resulting from $\mathrm{H}$ atom scattering with barrier crossing could not be observed at lower incidence energies.

Nevertheless, it can be clearly seen that the $P_{\mathrm{bc}} / P_{\mathrm{nbc}}$ ratio resulting from $\mathrm{H}$ atoms scattering from $(1 \times 1) \mathrm{Gr} / \mathrm{Ni}(111)$ tend to zero at lower normal incidence energies than the $P_{\mathrm{bc}} / P_{\mathrm{nbc}}$ ratio resulting from $\mathrm{H}$ atoms scattering from rot. $\mathrm{Gr} / \mathrm{Ni}(111)$

The best fit (colored lines) of the data points yields:

Table 6.1: Fitting parameters for Fig. 6.14

\begin{tabular}{lcc} 
& $(1 \times 1) \mathrm{Gr} / \mathrm{Ni}(111)$ & rot. $\mathrm{Gr} / \mathrm{Ni}(111)$ \\
\hline \hline$E_{0} / \mathrm{eV}$ & $0.23 \pm 0.07$ & $0.26 \pm 0.07$ \\
$k \cdot 10^{-7}$ & $-6.48 \pm 0.03$ & $-2.53 \pm 0.05$ \\
$t \cdot 10^{-6} / \mathrm{eV}$ & $6.1 \pm 0.4$ & $3.8 \pm 0.3$ \\
$n$ & $1.3 \pm 0.2$ & $1.4 \pm 0.2$ \\
$R S S$ & 0.0017 & 0.0019 \\
Adj. R-Square & 0.997 & 0.996
\end{tabular}

The quality of the fit is indicated by the small residual sum of square (RSS) and the adjusted $\mathrm{R}$ square value which is close to 1 .

As anticipated from the previous visual comparison of the energy distributions, the fit yields a lower adsorption threshold for $(1 \times 1) \mathrm{Gr} / \mathrm{Ni}(111)$ compared to rot. $\mathrm{Gr} / \mathrm{Ni}(111)$ and a much smaller value compared to $\mathrm{Gr} / \mathrm{Pt}(111)$. 


\subsection{Discussion}

Inelastic hydrogen atom scattering has been performed on two different graphene modifications on $\mathrm{Ni}(111)$. By comparison to pristine nickel, it could be established that a complete monolayer has been formed as no scattering originating from the sublayer metal was observed for both graphene samples.

The spectral features derived from the scattering experiments on $\mathrm{Gr} / \mathrm{Ni}(111)$ are similar to the previously observed features from studies on $\mathrm{Gr} / \mathrm{Pt}(111)$, as an quasi-elastic fast peak as well as a slow component corresponding to hydrogen atoms scattering from the graphene surfaces with barrier crossing have been observed. The determined thresholds for both graphene modifications on $\mathrm{Ni}(111)$ are more than $0.2 \mathrm{eV}$ smaller than for $\mathrm{Gr} / \mathrm{Pt}(111)$, which leads to the conclusion that the stronger graphene nickel interactions results in a partial $\mathrm{sp}^{3}$-hybridization and thus reduces the overall adsorption barrier.

Table 6.2: Experimentally determined adsorption thresholds at $300 \mathrm{~K}$.

\begin{tabular}{lc} 
& $E_{0} / \mathrm{eV}$ \\
\hline \hline$(1 \times 1) \mathrm{Gr} / \mathrm{Ni}(111)$ & $0.23 \pm 0.07$ \\
rot. $\mathrm{Gr} / \mathrm{Ni}(111)$ & $0.26 \pm 0.07$ \\
$\mathrm{Gr} / \mathrm{Pt}(111)$ & $0.47 \pm 0.02$
\end{tabular}

The lowest adsorption threshold was determined for $(1 \times 1) \mathrm{Gr} / \mathrm{Ni}(111)$, which would indicate a stronger interaction between the graphene layer and the nickel substrate. This study is therefore the first experimental evidence for significantly different properties and reactivities for both graphene modifications, that previously had only been postulated by theoretical models. $85 \mid 89$

The quasi-elastic component obtained from $\mathrm{H}$ atom scattering from graphene on $\mathrm{Ni}(111)$ at high incidence energies $\left(E_{\mathrm{i}}=1.92 \mathrm{eV}\right)$ showed a separation into two subcomponents, which was more pronounced for $(1 \times 1) \mathrm{Gr} / \mathrm{Ni}(111)$ than partially rotated $\mathrm{Gr} / \mathrm{Ni}(111)$. This separation seems to originate from dissimilar geometrical orientation of the carbon atoms on the $\mathrm{Ni}(111)$ substrate. Nevertheless, theoretical calculations are required in order to fully explain these observations.

In addition to the two aforementioned components, it was possible to detect an unexpected new third component that was not considered during the prior $\mathrm{H}$ atom scattering studies on $\mathrm{Gr} / \mathrm{Pt}(111)$. This component might have previously been missed due to either a too low resolution of the recorded spectra and/or its low signal intensity.

The significantly higher contribution of the third component for the $\mathrm{Gr} / \mathrm{Ni}(111)$ samples might be explained by the softened phonon modes of graphene on $\mathrm{Ni}(111)$ compared 
to free-standing graphene, especially for the out-of-plane vibrational mode (see Chapter 2.2.3. .8485 According to geometrical considerations, $\mathrm{H}$ atoms, which collide with the graphene surface, should predominantly transfer their inherent energy to this vibrational mode. The change in the phonon spectra might therefore result in a significantly higher phonon excitation probability for $\mathrm{Gr} / \mathrm{Ni}(111)$, and thus, explain the observed new third component. In order to support this thesis, investigations of the isotope effect might be beneficial as the efficiency of the phonon mode excitation should be dependent on the scattered isotope. In addition, it might be worthwhile investigating other metal substrates exhibiting a phonon spectrum different from the two extreme cases $\mathrm{Gr} / \mathrm{Ni}(111)$ and $\mathrm{Gr} / \mathrm{Pt}(111)$, which might yield a significant scattering energy shift for the third component. Nonetheless, theoretical calculations are essential in order to fully understand the scattering dynamics of this component. 


\section{Conclusion and Outlook}

In this thesis, a plethora of experimental and theoretical findings derived from inelastic hydrogen atom scattering on various surfaces using the Rydberg atom tagging machine have been discussed concerning fundamental energy transfer processes between a striking hydrogen/deuterium atom and a surface.

In the first main chapter 4 , in total six late transition $f c c$-metals have been investigated, in order to establish and optimize the experimental methodology as well as to further substantiate a novel advanced theoretical model developed by Janke et al. 128|150|165 which was subsequently extended by Kammler et al.129 for the general description of energy-transfer processes on metal surface treating electron-hole-pair excitation as a friction force. These combined experimental and theoretical studies revealed that the energy transfer for the investigated $f c c$-metals, primarily occurs via $e h p$ excitation and to a lower extent via phonon excitation. Nonetheless, it could be shown that in order to explain the experimentally determined isotope effects, both excitation processes have to be taken into account. These assumptions could be strengthened by hydrogen scattering experiments on an insulting xenon surface, for which solely phonon excitation is feasible. Further, these studies allowed to correlate the pronounced broadening of the scattering signal observed for the $f c c$-metals to ehp-excitation processes.

With respect to the nature of the six investigated $f c c$-metals, only marginally different scattering results have been obtained, which can predominantly be explained by the atomic mass difference of the $f c c$-metal surfaces. These findings are in full accordance with the theoretical model based on which a significant impact of the electronic properties could only be expected for metals with a drastically lower electron density. Due to the experimental preconditions for UHV measurements elemental aluminum would be best if not only viable option as lighter $f c c$-metal, which might show stronger differences in the scattering results compared to the studied transition metals so far. Comparison to theoretical simulations might further support the theoretical assumptions and demonstrate a relation between energy transfer inefficiencies and the electron density of metal surfaces. The theory further predicts a resolution of two or three different peaks in the energy 
loss distribution of $\mathrm{H} / \mathrm{D}$ atoms scattered from transition metal surfaces at lower surface temperature, which can be attributed to different scattering mechanisms such as singleor double-bounce scattering. 128 If this theoretical description of the thermal behavior is correct, for some of the peaks it might be possible to attribute them to distinct bounce events at surface temperatures of approximately $40 \mathrm{~K}$.

So far experiments were performed with incidence energies upto approximately $3 \mathrm{eV}$. The theory predicts for $E_{\mathrm{i}}>7 \mathrm{eV}$, a considerable shape change of the energy distribution, which includes larger nonadiabatic vs. adiabatic contributing effects. Thus, studies at high incidence energies might give further insight into the scattering dynamics.

After the investigation of metallic surfaces, hydrogen scattering experiments were for the first time performed on insulating bulk as well as thin-layer surfaces. For these types of surfaces adiabatic phonon excitation is the only feasible energy transfer process due to their inherent large band gap. As a result, primarily direct inelastic scattering has been observed during the experiments that can be described by simple collision models. In order to evaluate potential cooperative effects, various surface samples of insulating aluminium oxide with different layer thickness down to less than one monolayer have been prepared on a platinium substrate and subsequently examined. These experiments revealed no significant influence on the aluminum oxide layer thickness, thus, cooperative effects could not be detected. Further, subsequently performed scattering experiments on an oxygen covered $\mathrm{Pt}(111)$ surface did lead to the assumption that scattering almost exclusively occurs on the quasi-isolated oxygen atoms on the surface. This thesis could potentially be further substantiated by future hydrogen scattering experiments of sulfur on metal surfaces, as for the higher homologues of oxygen a similar scattering behavior could be anticipated, however the higher mass difference $(\mathrm{H} / \mathrm{S}$ vs. $\mathrm{H} / \mathrm{O})$ should lead to significantly lower energy loss, which could be easily simulated for a direct scattering process. Alternatively, low temperature scatterings could be performed, which might allow to observe a fine structure of the scattering distributions.

However, theoretical calculations are still required in order to support the assumptions made, especially regarding the role of the oxygen atoms during the scattering process.

Finally, in the third main chapter two modification of graphene on Ni(111), (1 x 1) oriented graphene and partially rotated graphene on $\mathrm{Ni}(111)$, have been intensively investigated and compared to previously obtained experimental and theoretical studies. Both investigated modifications showed certain similarities to $\mathrm{Gr} / \mathrm{Pt}(111)$, however, the detailed examination of the obtained data revealed two unexpected new features: two 
subcomponents for the first quasi-elastic peak and the presence of a new third scattering component that previously had not been considered for $\mathrm{Gr} / \mathrm{Pt}(111)$.

In order to gain a more comprehensive understanding of these features, additional theoretical simulations as well as further experimental studies (e.g., out-of-plane measurements, azimuth dependent measurements at higher incidence energies, studies of the deuterium effect or temperature dependent/low temperature measurements as well as graphene on different metal substrate) seem necessary.

Furthermore, these experiments revealed a significantly lower absorption threshold for both $\mathrm{Gr} / \mathrm{Ni}(111)$ modification compared to $\mathrm{Gr} / \mathrm{Pt}$, which could be explained by the stronger interaction between the graphene monolayer and the nickel substrate. Although their barrier height differences are smaller by an order of magnitude, it was also possible to identify a significantly smaller adsorption threshold for $(1 \times 1)$ compared to rot. $\mathrm{Gr} / \mathrm{Ni}(111)$. This observation could be explained by a stronger graphene-substrate interactions due to a more preferential geometric arrangement of the carbon-atoms. This therefore represents the first experimental evidence for different properties and thus reactivities of both graphene species due to their different geometric arrangement. As aforementioned, theoretical simulations for both $\mathrm{Gr} / \mathrm{Ni}(111)$ modifications seem necessary in order to conclusively explain the obtained experimental results. Nonetheless, these findings again demonstrate the potential of the Rydberg atom tagging technique for the investigation of complex surfaces which gives direction for the further development of theoretical models for the comprehensive understanding of surface dynamics. 



\section{Appendix A}

\section{Conversion from TOF distribution to energy distribution}

The time of flight of the scattered H/D atoms between the tagging area and the detector was recorded in a period of 0 to $100 \mu \mathrm{s}$ with a bin width of $8 \mathrm{~ns}$. The VUV tagging laser light generates a counting signal at $\sim 370 \mathrm{~ns}$, that can be used as a marker while the scattered $\mathrm{H} / \mathrm{D}$ atoms travel through the tagging region. The distance between the tagging area and the detector $(d \approx 255.3 \mathrm{~mm})$ was determined by measuring the TOF of the $\mathrm{H} / \mathrm{D}$ atom beam.

In general, HRTOF distributions of the scattered $\mathrm{H} / \mathrm{D}$ atoms were recorded within one minute by a waggle scan varying the time delay between the photolysis and tagging laser pulse. The time delay of the laser pulses was varied within a time frame of $\mathrm{H} / \mathrm{D}$ atoms passing through the distance between the photolysis and tagging region. Subsequently, background measurements were performed for one minute by reducing the time delay between the photolysis and tagging laser to $6 \mu$ s ere H/D atoms reach the tagging point. The flight time of the atoms from the dissociation point to the tagging point depends on their inherent kinetic energy and varies between 7 and $17 \mu \mathrm{s}$.

In order to gain a suitable signal to noise ratio, the measurement procedure was repeated in the performed experiment for $0.5-3 \mathrm{~h}$. Total TOF distributions of the scattered $\mathrm{H} / \mathrm{D}$ atoms were then created by summing up the TOF distributions after background correction.

Fig. 7.1 shows a representative total TOF distribution spectrum (black dots) of $\mathrm{H}$ atoms scattered from $\mathrm{Au}(111)$ by plotting the count signal of the scattered $\mathrm{H}$ atoms against their time of flight.

The measured density dependent TOF distribution can further be converted into a total flux-weighted translational energy distribution applying proper Jacobian and density to flux corrections according to Eq. 3.11. Fig. 7.2 presents the corresponding energy distribution of the scattered $\mathrm{H}$ atoms (black dots).

The energy dependent $\mathrm{H}$ atom flux $F(E)$ plotted against the final kinetic energy $E_{\text {kin }}$ of the scattered $\mathrm{H}$ atoms was normalized using the integral of the overall signal. Due 


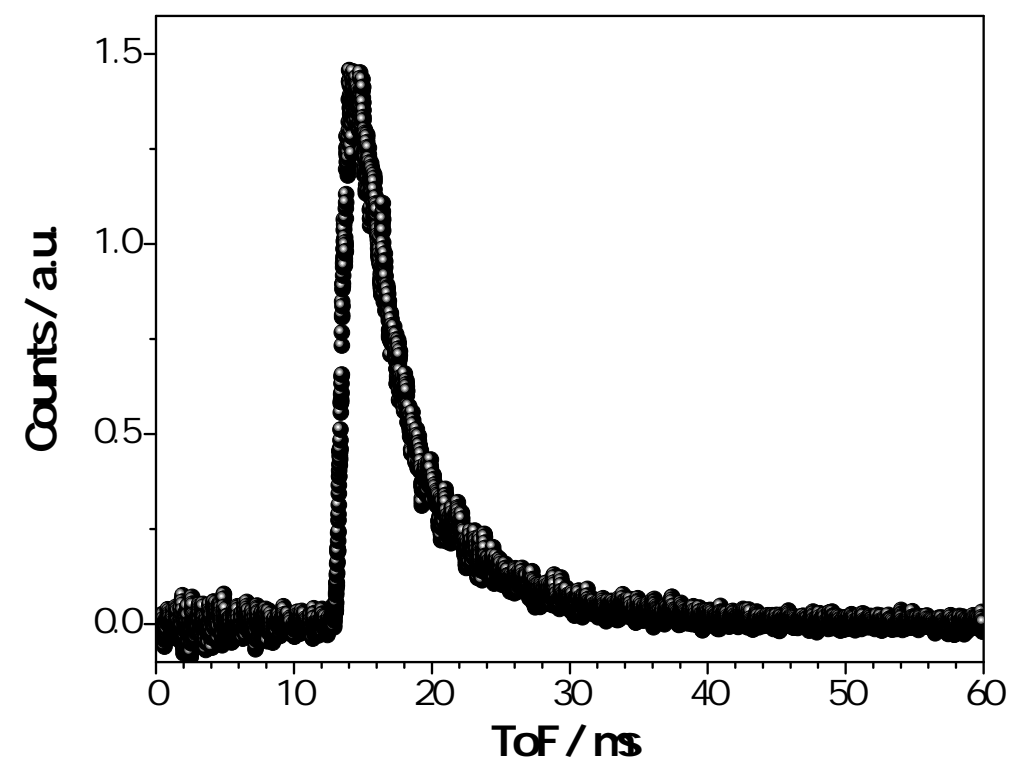

Figure 7.1: TOF distribution spectrum of $\mathrm{H}$ atoms scattered from a $\mathrm{Au}(111)$ surface at $E_{\mathrm{i}}=$ $1.92 \mathrm{eV}$ and $\vartheta_{\mathrm{i}}=\vartheta_{\mathrm{i}}=45^{\circ}$ with a bin width of $8 \mathrm{~ns}$.

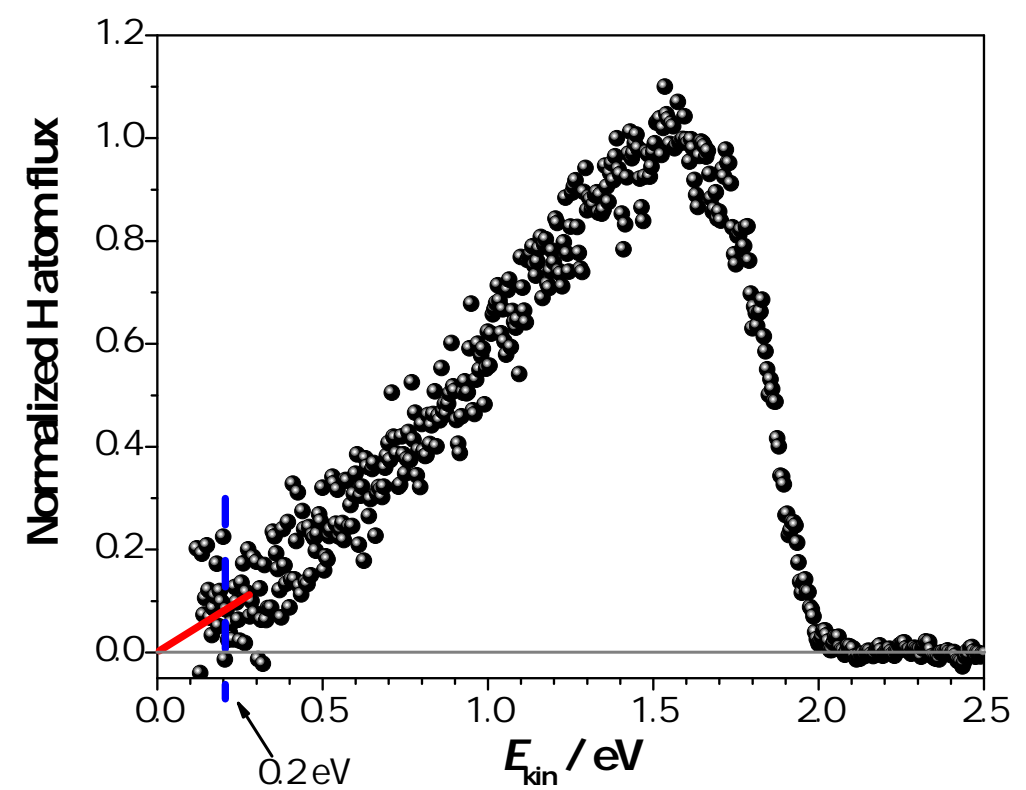

Figure 7.2: Translational energy distribution spectrum of $\mathrm{H}$ atoms scattered from a $\mathrm{Au}(111)$ surface at $E_{\mathrm{i}}=1.92 \mathrm{eV}$ and $\vartheta_{\mathrm{i}}=\vartheta_{\mathrm{i}}=45^{\circ}$ applying a proper Jacobian and density to flux conversion. The red line indicates a linear fit in order to estimate the $\mathrm{H}$ atom flux probability at kinetic energies between 0 and $0.2 \mathrm{eV}$. 
to the Jacobian transformation, the noise level at kinetic energies below $0.2 \mathrm{eV}$ (dashed blue line) is very high with $\mathrm{H}$ atom flux signals close to zero. Therefore, no data points were recorded for kinetic energies between 0 and $0.2 \mathrm{eV}$. For the purpose of determining comparable overall integrals, the signal of the interval $[0,0.2 \mathrm{eV}]$ was fitted by a linear function (red line). The $\mathrm{H}$ atom flux value at $0.2 \mathrm{eV}, F(0.2 \mathrm{eV})$, was approximated by calculating the mean value of the last 20 data point at $E_{\text {kin }} \geq 0.2 \mathrm{eV}$. The normalized $\mathrm{H}$ atom flux $F_{\mathrm{n}}(E)$ can then be derived from the following equation:

$$
F_{\mathrm{n}}(E)=\frac{F(E)}{\int_{0.2 \mathrm{eV}}^{E_{\mathrm{i}}+1} F(E) \mathrm{d} E+F(0.2 \mathrm{eV}) \cdot 0.1 \mathrm{eV}}
$$

The final average kinetic energy of the scattered $\mathrm{H}$ atoms $\left\langle E_{\mathrm{fin}}\right\rangle$ is obtained calculating the weighted arithmetic mean of the data points:

$$
\left\langle E_{\mathrm{fin}}\right\rangle=\frac{\sum_{\mathrm{i}=1}^{n} E_{\mathrm{kin}, \mathrm{i}} \cdot F\left(E_{i}\right)}{\sum_{\mathrm{i}=1}^{n} F\left(E_{i}\right)}
$$

The translational kinetic energy loss $E_{\text {loss }}$ of the scattering $\mathrm{H} / \mathrm{D}$ atoms is estimated by subtracting the kinetic energy of the incidence H/D atoms from the kinetic energy of the scattered $\mathrm{H} / \mathrm{D}$ atoms.

$$
E_{\text {loss }}=E_{\text {fin }}-E_{\mathrm{i}}
$$

Thus, it follows for the average kinetic energy loss of the scattered $\mathrm{H}$ atoms:

$$
\left\langle E_{\mathrm{loss}}\right\rangle=\left\langle E_{\mathrm{fin}}\right\rangle-E_{\mathrm{i}}
$$

The relative average final kinetic energy is normalized to the incidence energy $\left\langle E_{\text {loss }}\right\rangle / E_{\mathrm{i}}$. 



\section{Appendix B}

\section{Angular distribution correction}

The angular distribution of a specified incidence energy and angle represents the count signal of the scattered H/D atoms with respect to the scattering angle. The count signal is obtained from an integral of the density-weighted TOF distribution over a certain TOF interval. It should be noted, that the recorded angular distribution overestimates the count rate at higher scattering angles as with increasing scattering angle the opening angle of the detector covers an increasing area on the surface:

The opening angle of the detector perpendicular to the scattering plane is limited by the detector aperture and the diameter of the MCP and does not change with detector position. In contrast, the opening angle of the detector in-plane to the $\mathrm{H} / \mathrm{D}$ atom beam is limited by the focus diameter of the tagging laser as well as the detector aperture and depends on the position of the detector with respect to the sample surface.

Fig 7.3 illustrates the opening angle of the detector with respect to the scattering angle $\vartheta_{\text {s }}$ in-plane perpendicular to the tagging laser. For $\vartheta_{\mathrm{s}}=0^{\circ}$ the opening angle of the detector covers an elliptical area on the surface with a diameter in the scattering plane marked as

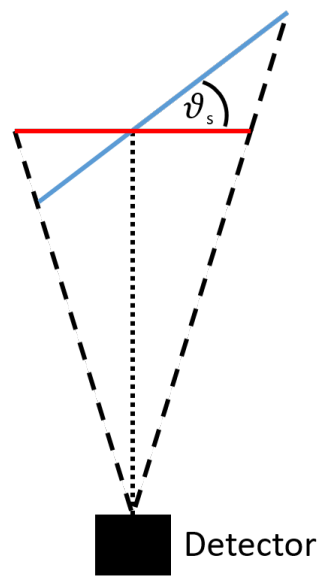

Figure 7.3: Scheme of the opening angle (black dashed lines) of the detector covering the surface (red and blue line) with respect to the scattering angle, $\vartheta_{\mathrm{s}}$. 


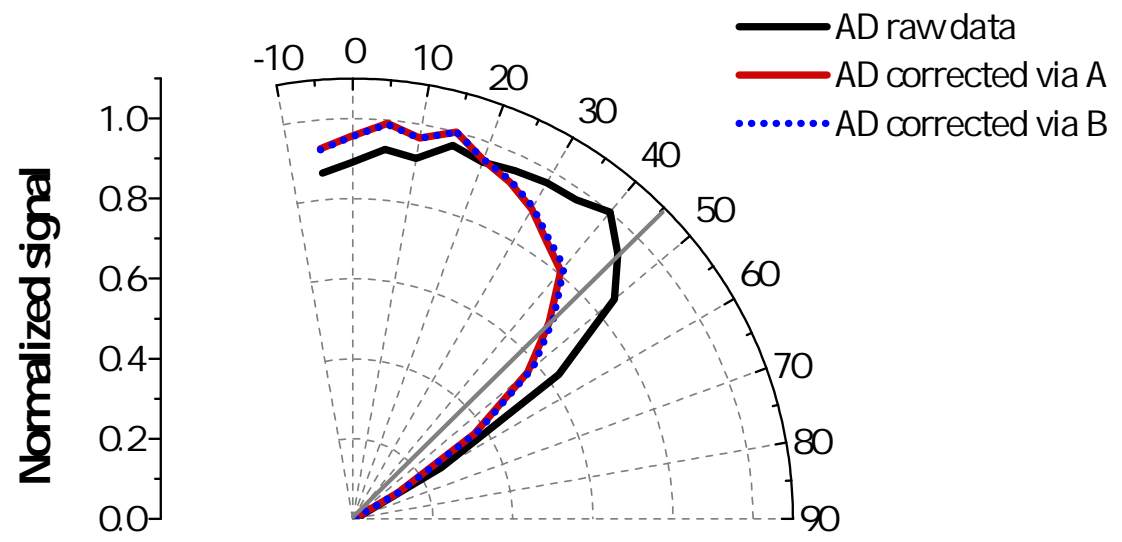

Figure 7.4: Raw (black line) and corrected (red line and blue dotted line) angular distribution spectra of $\mathrm{H}$ atoms scattered from a $\mathrm{Au}(111)$ surface at $E_{\mathrm{i}}=3.33 \mathrm{eV}$ and $\vartheta_{\mathrm{i}}=45^{\circ}$.

red line. With a scattering angle of $\vartheta_{\mathrm{s}}>0^{\circ}$, the diameter (blue line) increases.

For the correction, a few assumptions have to be made. First, it is assumed that the $\mathrm{H} / \mathrm{D}$ atom beam covers the entire crystal. Second, the number of H/D atom collision events with the surface is equal for all surface positions. As a result, the count rate is proportional to the surface area covered by the detector.

In a first approximation (Correction A) the recorded raw count signal $I_{\text {raw }}\left(\vartheta_{\mathrm{s}}\right)$ can be corrected by the cosine of the scattering angle leading to the corrected count signal $I_{\text {cor }}\left(\vartheta_{\mathrm{s}}\right)$.

$$
I_{\text {cor }}\left(\vartheta_{\mathrm{s}}\right)=I_{\text {raw }}\left(\vartheta_{\mathrm{s}}\right) \cdot \cos \left(\vartheta_{\mathrm{s}}\right)
$$

A more sophisticated consideration (Correction B) of the geometrical experimental setup was also performed. The principal code used in Mathematica in order to perform Correction $\mathrm{B}$ is given at the following page.

Figure 7.4 shows raw data from an angular distribution measurement (black line) of $\mathrm{H}$ atoms scattered from $\mathrm{Au}(111)$ at $E_{\mathrm{i}}=3.33 \mathrm{eV}$ and $\vartheta_{\mathrm{i}}=45^{\circ}$ normalized to the signal maximum. The blue dotted line and solid red line represent the corrected angular distributions applying Correction A and B, respectively. The comparison between the raw and the corrected distributions clearly demonstrates that a correction of the raw scattering signal has to be performed as the measured angular distribution (raw Data) significantly overestimates the scattering signal at scattering angles higher than $30^{\circ}$.

No significant differences are visible between both corrected data sets leading to the conclusion that Correction A can be applied for data adjustment of the measured angular distributions. 
$\mathrm{DL}=\mathbf{0 . 5}$; "Diameter Tagging-Laser, $<1 \mathrm{~mm} / \mathrm{mm} "$

DB = 5; "Diamter detector aperture / mm"

DK $=10$; "Diameter crystal"

DBB = 50; "Diameter MCP"

d1 = 165.3; "Distance Aperture-MCP / mm"

d2 = 90; "Distance Aperture-Tagging point /mm"

d3 = 0.7; "Distance Tagging-Surface"

Data "recorded raw AD Data"

$\alpha$, "Sample Polar Angle / rad"

$\vartheta$, "Scattering angle / rad"

$\gamma=90-\vartheta$, "relative Angle: Surface-Detector"

$\mathbf{h}=\mathbf{d} 3 / \operatorname{Cos}[\vartheta]$, "relative mean distance Tagging-Surface viewpoint Detector" LKosinus

$a=d 3 * \operatorname{Tan}[\theta], \quad$ "Distance intercept $d 4-h /$ Surface"

LTangente

$\delta=\operatorname{ArcTan}[(\mathrm{DL}+\mathrm{DB}) / 2 / \mathbf{d} 2]$, "Opening angle detector in-plane"

LArkustangens LÖffnen

$\mathrm{DI}=\mathrm{DL}+\mathbf{h} / \mathbf{d} 2 *(\mathrm{DB}+\mathrm{DL})$, "Diamter opening angle out-of-plane at dist. $\mathrm{h} "$

r1 $=\mathrm{DI} / 2 * \operatorname{Sin}[90+\delta] / \operatorname{Sin}[\gamma-\delta]$, "Upper Radius of $\delta "$

LSinus LSinus

r2 $=\mathrm{DI} / 2 * \operatorname{Sin}[90-\delta] / \operatorname{Sin}[\gamma+\delta], \quad$ "Lower Radius of $\delta "$

$$
\text { LSinus LSinus }
$$

$01=\mathrm{DK} / \mathbf{2}-\mathbf{a}-\mathbf{r} \mathbf{1}$, "must be $>0$, otherwise radius of $\delta>\mathrm{DK} / 2$ "

$\mathrm{DI} 2$ = DB + d3 / d1 * (DB + DBB), "Diameter opening angle out-of-plane at surface"

Okorr = ReplacePart $\left[01\right.$, Position $\left.\left[01, b_{-} / ; b>0\right] \rightarrow 0\right]$,

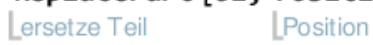

$\mathrm{A} 1=\pi * \mathrm{DI} 2 *(\mathrm{r} \mathbf{1}+\mathrm{r} \mathbf{2}) / 4$, "Observed surface area if $\delta<\mathrm{DK} "$

$\mathrm{A} 2=\pi * \mathrm{DI} 2 *(\mathbf{r} \mathbf{1}+$ Okor $r+\mathrm{r} 2) / 4$, "Observed surface area if $\delta>\mathrm{DK} "$

Discorrect $=$ Data $/$ A2, "Correction of raw AD data" 



\section{Appendix C}

Graphene 2D translational energy and angular distributions 

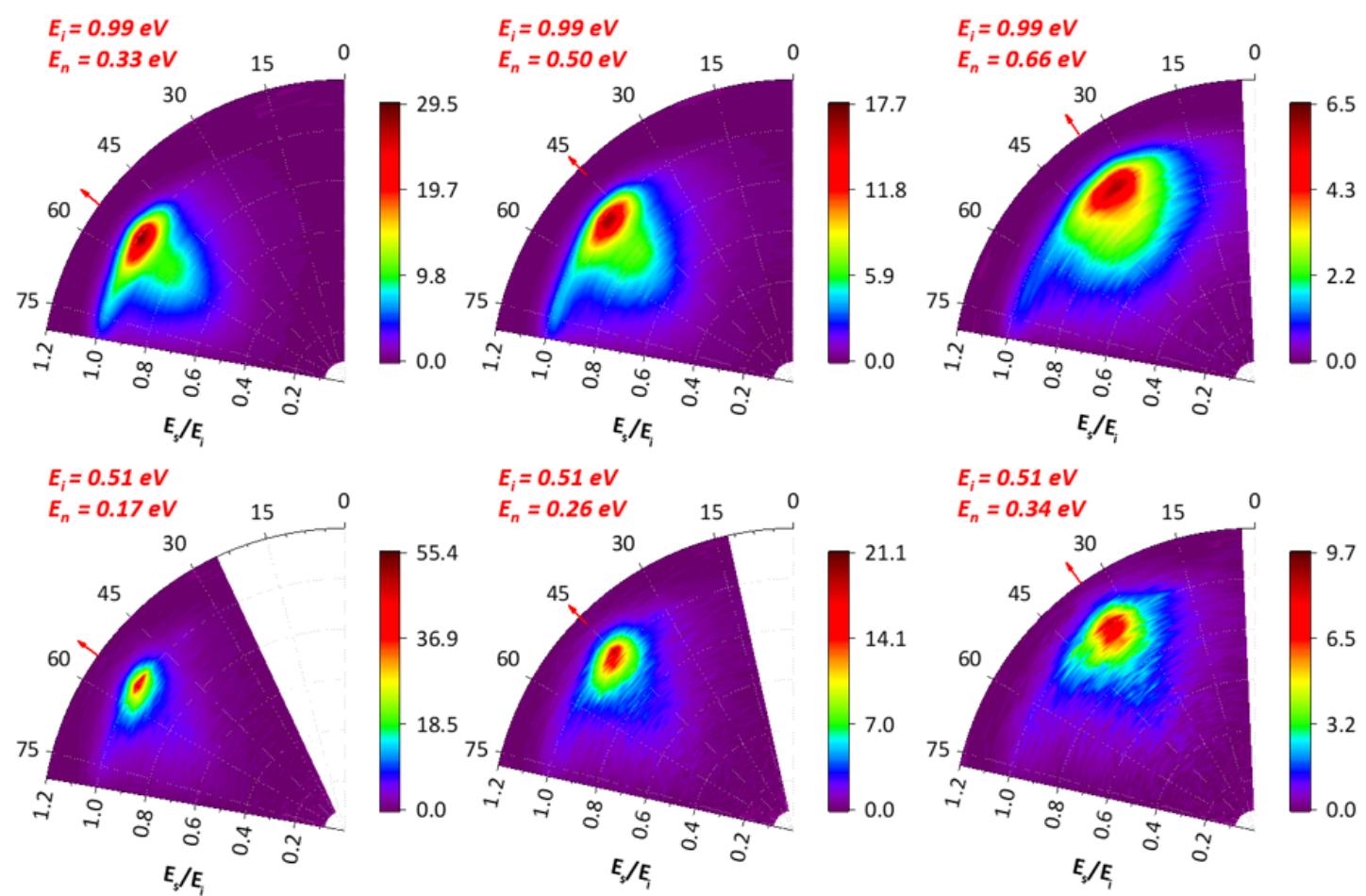

Figure 7.5: Selection of 2D translational energy and scattering angular distributions of $\mathrm{H}$ atoms scattered from rot. $\mathrm{Gr} / \mathrm{Ni}(111)$ measured at different incidence angles at $E_{\mathrm{i}}=0.99 \mathrm{eV}$ (upper panels) and $0.51 \mathrm{eV}$ (lower panels). The red arrows mark the specular scattering angle. The figures are labeled by the incidence energy and corresponding normal incidence energy. 

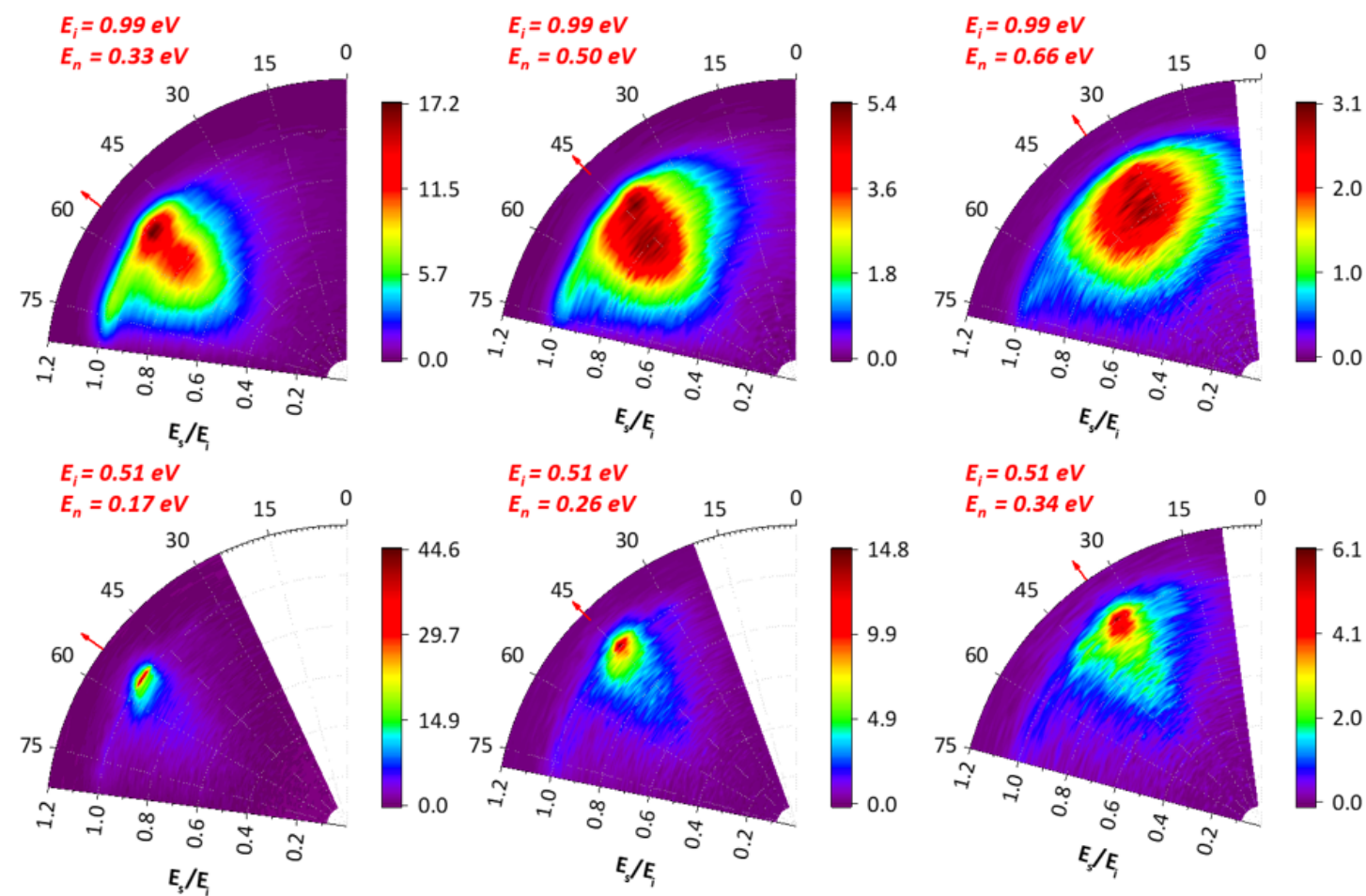

Figure 7.6: Selection of $2 \mathrm{D}$ translational energy and scattering angular distributions of $\mathrm{H}$ atoms scattered from $(1 \times 1) \mathrm{Gr} / \mathrm{Ni}(111)$ measured at different incidence angles at $E_{\mathrm{i}}=0.99 \mathrm{eV}$ (upper panels) and $0.51 \mathrm{eV}$ (lower panels). The red arrows mark the specular scattering angle. The figures are labeled by the incidence energy and corresponding normal incidence energy. 



\section{Appendix D}

\section{Graphene data analysis}

The energy distribution spectra were fitted by Gaussian distribution fits with respect to the incidence and scattering angle in order to separate the different scattering components from one another. The in-plane scattering intensity of each component was calculated by integration over the respective energy distributions. In order to estimate the 3D scattering intensity of each individual component, it was assumed that the angular distribution of the separated components are spherical for in- and out-of-plane measurements. The used Mathematica code is provided in this chapter. As an example, fits of experimental data for rotated graphene on $\mathrm{Ni}(111)$ obtained at an incidence angle of $52^{\circ}$ are shown. 
(*Input Data, Figures: Angular distribution, Energy distribution and 3D plot*)

Tabelle = "Gr120";

Eingabe = Import

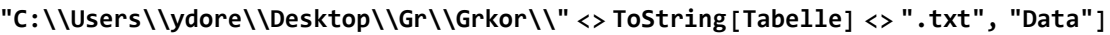

SDL = Dimensions [Eingabe] [ [1] ]

Surface $=$ "rGN";

$i=11 ;\left(*\right.$ Data of incidence angle $52^{\circ} *$ )

$\mathrm{nD}=\mathbf{i}$;

pi $=$ TextString $[$ Eingabe $[[i, 7]]]$;

ps = TextString $[$ Eingabe $[[i, 8]]]$;

Ekinat $=$ TextString $[$ Eingabe $[i, 2]]]$;

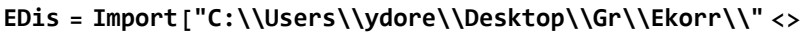

ToString [ Eingabe $[[i, 1]]$, Eingabe $[[i, 2]]$, Eingabe $[[i, 8]]$, Eingabe $[i, 9]]\}]<>$

"_" <> ToString [Eingabe [ [i, 12] ] ] <> ".dat", "Table"];

Angle $=$ Drop [EDis [ [1] ], 1];

$E D=\operatorname{Drop}[$ Transpose $[$ Drop $[$ EDis, 1] $], 1]$;

EDkorr = ReplacePart $\left[E D\right.$, Position $\left[E D, b_{-} /\right.$; b < $\left.\left.<\right] \rightarrow \theta\right]$;

EDM = Transpose [EDkorr $]$;

DD = Dimensions [EDM] ;

EDsuma $=$ Total [ED] * Abs [Angle [ [1] ] - Angle [ [-1] ] ]/Dimensions [Angle] [ [1] ];

(*0liver eData*)

EDsum = ReplacePart [EDsuma, Position [EDsuma, $\left.b_{-} / ; \mathbf{b}<0\right] \rightarrow 0$ ];

Energy $=$ Take $[$ Transpose $[$ Drop $[$ EDis, 1] $], 1][[1]]$;

EDsum1 $=$ Transpose $[\{$ Energy, EDsum $\}]$; (*0liver eData + Energy*)

$\operatorname{AD1}=\{0\}$;

For $[a=1, a \leq$ Dimensions [ED] [ [1] ], $a++,\{$

$\mathrm{g}=\mathrm{ED}[\mathrm{a}]]$,

$\mathrm{F1}=\operatorname{Sum}[\mathrm{g}[[\mathrm{b}]],\{\mathrm{b}, \mathbf{1}, \operatorname{Dimensions}[\mathrm{g}][[1]]\}] *$ Abs [Energy [ [1] ] - Energy [ [2] ] ] ;

AD1 = Append $[A D 1, F 1]$;

\}; ] ;

$A D=\operatorname{Drop}[A D 1,1] ;(* a D a t a *)$

aDataMatrix = Table $[0,\{x, 1, \operatorname{sDL}\},\{y, 1$, Dimensions [Angle $][1]]\}]$;

eDataMatrix $=$ Table $[0,\{x, 1, \operatorname{sDL}\},\{y, 1$, Dimensions [Energy $][[1]]\}]$;

iData $=$ Total [AD2] * Abs [Angle [ [1] ] - Angle [ [-1] ] ]/Dimensions [Angle] [ [1] ] ;

mData $=$ Max $[E D] ;$

AD2 $=$ Transpose $[\{$ Angle,$A D\}] ;(*$ Olivers aData + Angle $*)$

iAngle = Eingabe $[[i, 8]]$;

$\mathrm{dE}=$ Abs [Energy [ [1] ] - Energy [ [2] ] ];

$\mathrm{dA}=$ Abs [Angle [ [1] ] - Angle [ [-1]]]/Dimensions [Angle] [ [1] ];

List [ListPlot [AD2, PlotStyle $\rightarrow$ \{Hue [1], PointSize [0.02] \}],

ListPlot [EDsum, PlotStyle $\rightarrow$ \{Hue [0.7], PointSize [0.02] \}] ]

$\mathbf{k}=$ Flatten [Transpose [Table [Energy, $\{\mathbf{i}, 1$, Dimensions [Angle] [ [1] ] $\}]]$ ];

1 = Flatten [Table [Angle, $\{\mathbf{i}, 1$, Dimensions [Energy] [ [1] ] $\}]$;

heds = Flatten [EDM];

ED3D $=$ Transpose $[$ List $[1, k$, heds $]]$;

ConPlot $=$ ListContourPlot $[$ ED3D, GridLines $\rightarrow$ False, $\{1\}\}$,

GridLinesStyle $\rightarrow$ Directive [Gray, Dashed] , ContourStyle $\rightarrow$ None,

Colorfunction $\rightarrow($ If [\# $\leq 0.01$, Purple, Blend ["Rainbow", \#] ] \&),

PlotLegends $\rightarrow$ Automatic, PlotRange $\rightarrow$ Full, Full, $\{0$, Full $\}$,

PlotLabel $\rightarrow$ Surface Ekinat pi, Clippingstyle $\rightarrow$ \{Blue, Red ,

Labelstyle $\rightarrow$ Directive [Bold, Black, 12] , 
LabelStyle $\rightarrow$ Directive [Black, 16] , FrameStyle $\rightarrow$ Directive [Black, 14], FrameLabel $\rightarrow\left\{" \theta / \circ ", " E_{k i n} / E_{i} "\right\}$, Frame $\rightarrow\{$ True, True, True, True $\left.\}\right]$; Print [

ConPlot]

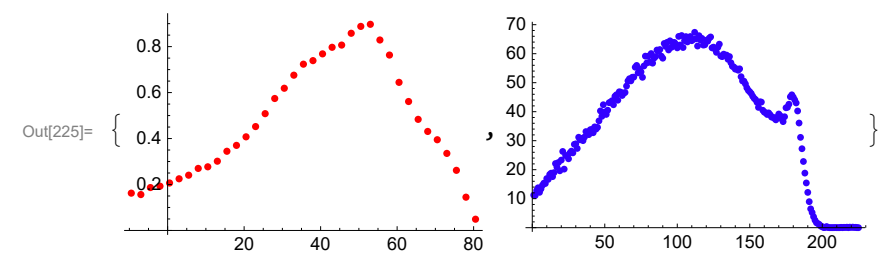

1.9252 rGN

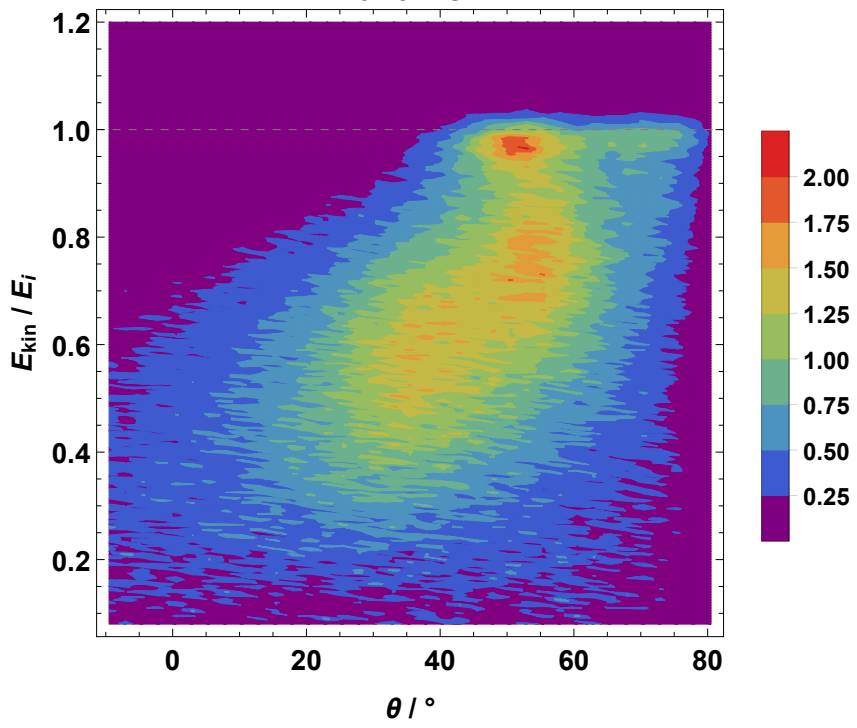

$\ln [611]=(*$ Fit of the energy distribution

summed over all scattering angles*)

emodel1 $=$ ea $1 * \operatorname{Exp}\left[-(x-\text { eb1 })^{\wedge} 2 /(2 *\right.$ ec1 $\left.)\right]$;

emode12 = ea1 $* \operatorname{Exp}\left[-(x-\mathrm{eb} 1)^{\wedge} 2 /(2 *\right.$ ec1 $\left.)\right]+\operatorname{ea} 2 * \operatorname{Exp}\left[-(x-\mathrm{eb} 2)^{\wedge} 2 /(2 * \mathrm{ec} 2)\right] ;$

emodel $3=\operatorname{ea} 1 * \operatorname{Exp}\left[-(x-\text { eb1 })^{\wedge} 2 /(2 *\right.$ ec1 $\left.)\right]+$

ea $2 * \operatorname{Exp}\left[-(x-\text { eb2 })^{\wedge} 2 /(2 *\right.$ ec2 $\left.)\right]+$ ea $3 * \operatorname{Exp}\left[-(x-\text { eb3 })^{\wedge} 2 /(2 *\right.$ ec3 $\left.)\right]$;

emode14 $=$ ea1 $* \operatorname{Exp}\left[-(x-\text { eb1 })^{\wedge} 2 /(2 *\right.$ ec1 $\left.)\right]+\operatorname{ea} 2 * \operatorname{Exp}[-(x-e b 2) \wedge 2 /(2 *$ ec2 $)]+$ ea $3 * \operatorname{Exp}\left[-(x-\text { eb3 })^{\wedge} 2 /(2 *\right.$ ec3 $\left.)\right]+$ ea $4 * \operatorname{Exp}\left[-(x-\text { eb4 })^{\wedge} 2 /(2 *\right.$ ec4 $\left.)\right]$;

emodel $15=\operatorname{ea} 5 * \operatorname{Exp}\left[-(x-\text { eb5 })^{\wedge} 2 /(2 *\right.$ ec5 $\left.)\right]+$ ea1 $* \operatorname{Exp}\left[-(x-\text { eb1 })^{\wedge} 2 /(2 *\right.$ ec1 $\left.)\right]+$ ea2 $2 \operatorname{Exp}\left[-(x-\text { eb2 })^{\wedge} 2 /(2 *\right.$ ec2 $\left.)\right]+$ ea $3 * \operatorname{Exp}\left[-(x-\text { eb3 })^{\wedge} 2 /(2 *\right.$ ec3 $\left.)\right]+$ ea4 * $\operatorname{Exp}\left[-(x-\text { eb4 })^{\wedge} 2 /(2 *\right.$ ec4 $\left.)\right]$;

eFitData $=$ FindFit $\left[\{\text { Energy, EDsum }\}^{\top},\{\right.$ emode15,

$\{0 \leq$ ea5 < Max [EDsum], $\theta \leq$ ea $1<\operatorname{Max}$ [EDsum], $0 \leq$ ea $2<\operatorname{Max}$ [EDsum],

$0 \leq \mathrm{ea3}<\operatorname{Max}$ [EDsum], $0 \leq \mathrm{ea4}<\operatorname{Max}$ [EDsum], $0.1<\mathrm{eb5}<0.38,0.2<\mathrm{eb} 1<0.6$,

$0.75<\mathrm{eb} 2<0.8,0.9<\mathrm{eb} 3<0.93,0.98<\mathrm{eb} 4<1.1,0.01<\mathrm{ec} 5<0.1$,

$0.01<\mathrm{ec} 1<0.1,0.012<\mathrm{ec} 2<0.013,0.002<\mathrm{ec} 3<0.005,0.0004<\mathrm{ec} 4<0.0005\}\}$, \{ea5, eb5, ec5, ea1, eb1, ec1, ea2, eb2, ec2, ea3, eb3, ec3, ea4, eb4, ec4\}, $x$, MaxIterations $\rightarrow$ 500] (*Model?*) 


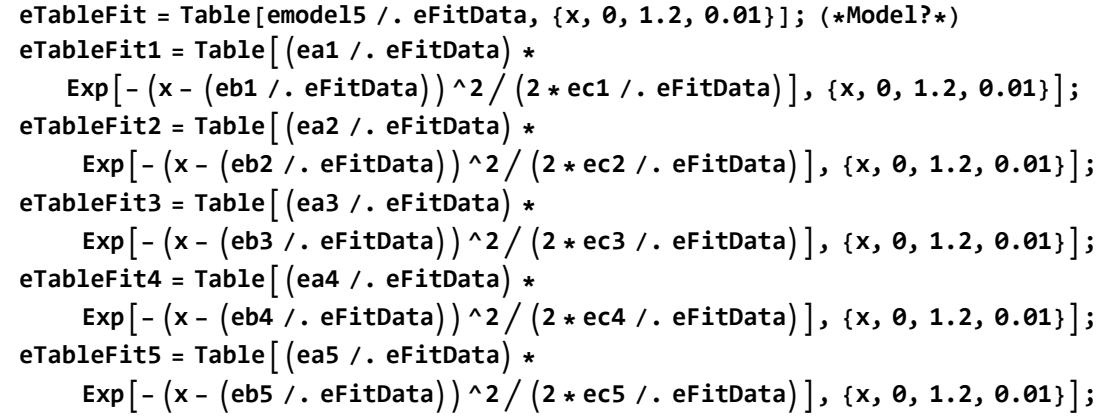


4 | FitGr1_Nifirstappendix.nb

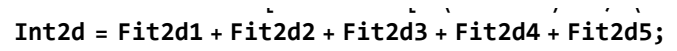

IntList2d $=\operatorname{List}[\mathrm{pr}, \operatorname{Cos}[\mathrm{pr} / 180 * \pi] * \operatorname{Eingabe}[[i, 2]]$,

$\operatorname{Cos}[p c / 180 * \pi] *$ Eingabe $[i, 2]]$, Int2d, Fit2d1, Fit2d2, Fit2d3, Fit2d4, Fit2d5];

$p r=\operatorname{Eingabe}[[i, 7]] ;$

pc $=$ Eingabe $[[i, 8]]$;

Ges2d = \{IntList2d $\}$

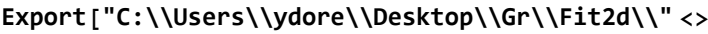

ToString [ $\{$ Surface, Ekinat, pi, ps\}] <> ".dat", Ges2d] ;

EDFits = List [Energy, EDsum, EFit, eTableFit, eTableFit1,

eTableFit2, eTableFit3, eTableFit4, eTableFit5];

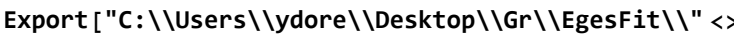

ToString [ $\{$ Surface, Ekinat, pi, ps\}] <> ".dat", EDFits];

Out[66] $=\{$ ea5 $\rightarrow 27.9928$, eb5 $\rightarrow 0.307041$, ec5 $\rightarrow 0.0291436$, ea1 $\rightarrow 50.4274$, eb1 $\rightarrow 0.578792$, ec1 $\rightarrow 0.0224215$, ea $2 \rightarrow 33.0221$, eb2 $\rightarrow 0.783446$, ec $2 \rightarrow 0.012$, ea3 $\rightarrow 18.4433$, eb3 $\rightarrow 0.93$, ec3 $\rightarrow 0.00267039$, ea $4 \rightarrow 25.9127$, eb4 $\rightarrow 0.981792$, ec $4 \rightarrow 0.0005\}$

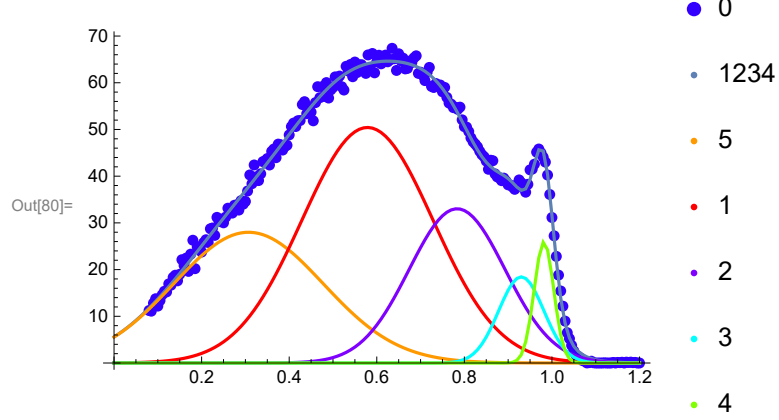

Out $[81]=\{43.4086,18.9262,9.06692,2.38899,1.45241,11.5742\}$

Out[106] $=\{\{52,1.18207,1.30944,43.3811,18.926,9.06681,2.38899,1.45241,11.5469\}\}$ 


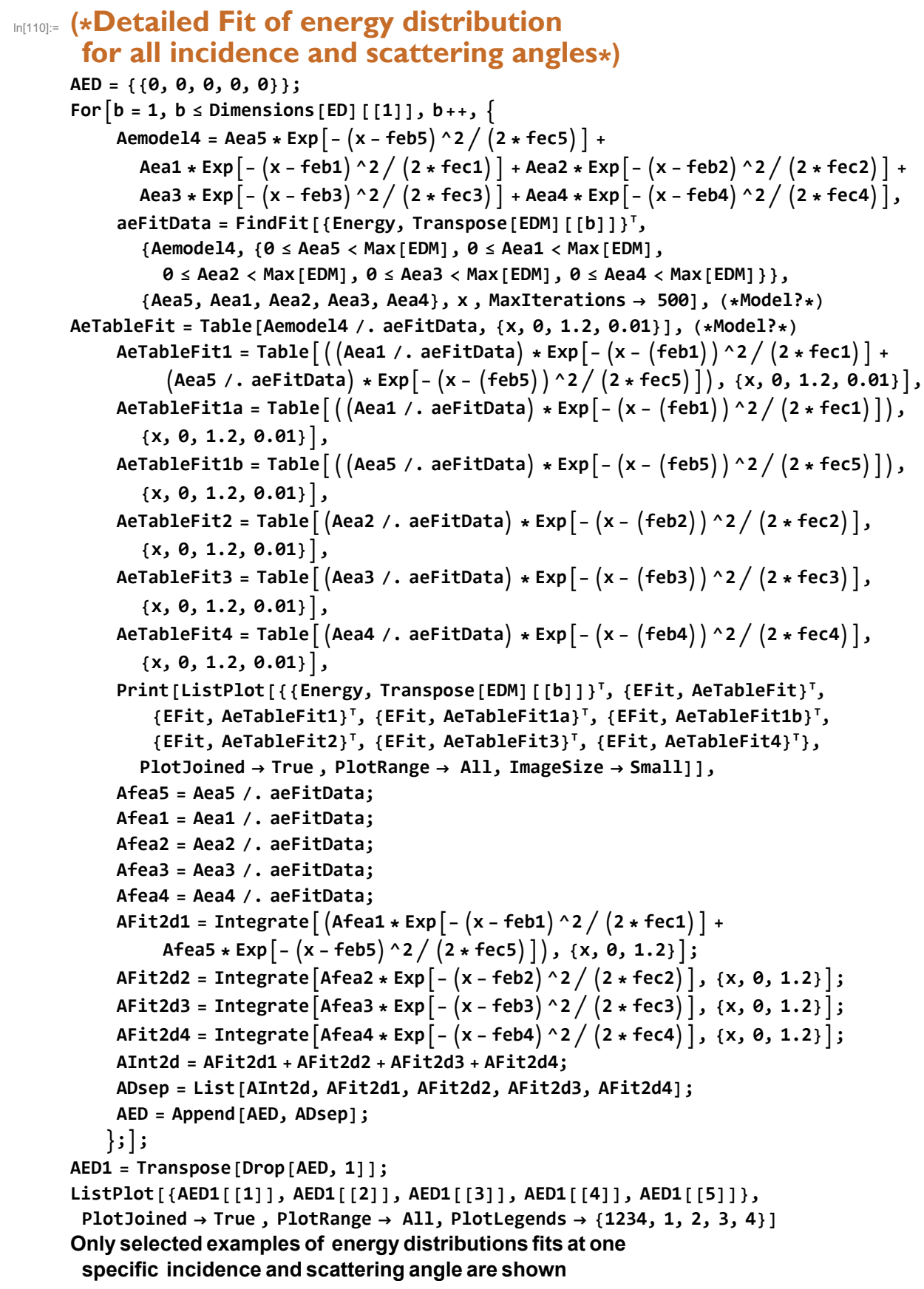


6 | FitGr1_Nifirstappendix.nb
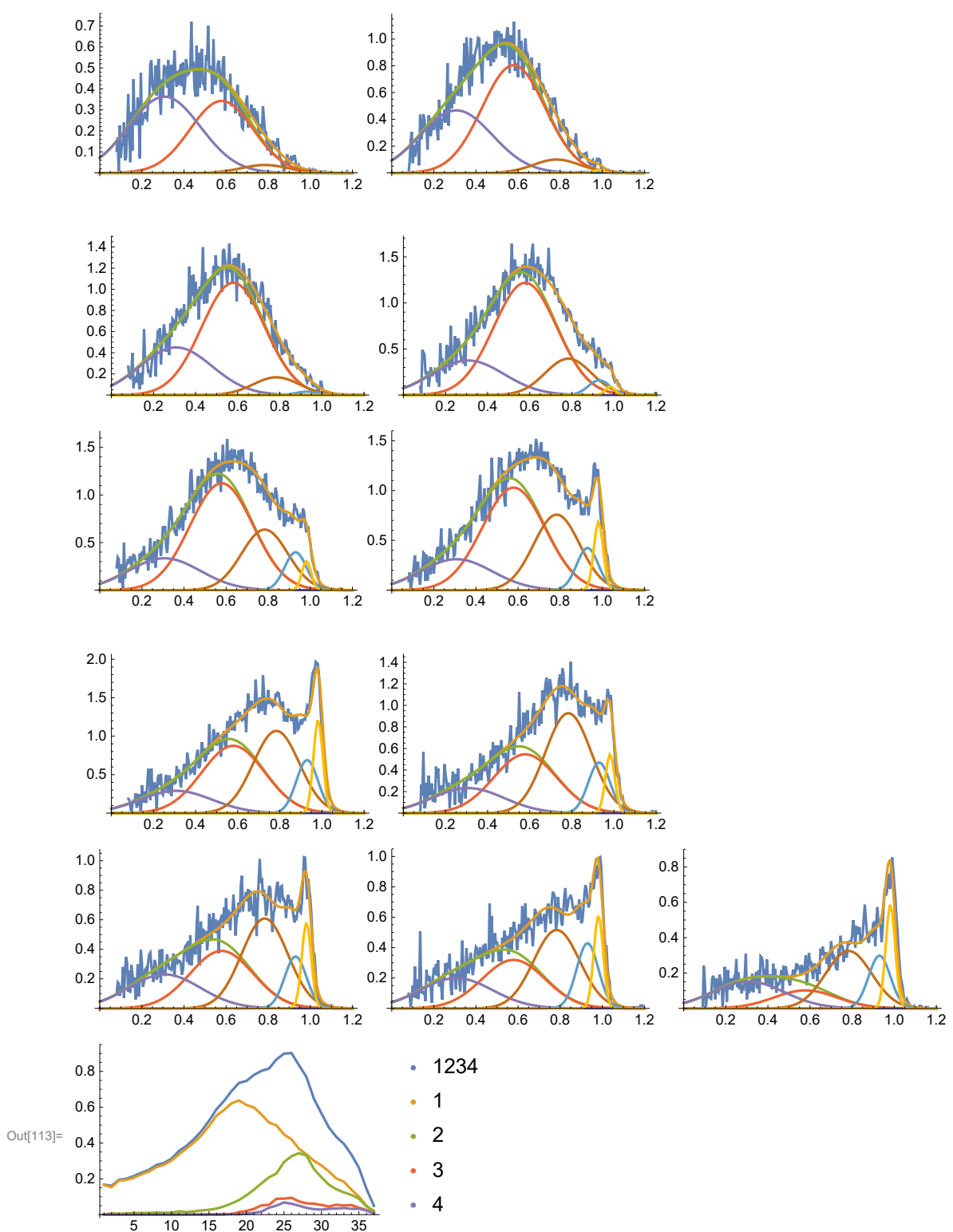

- 1234

- 1

- 2

- 3

\section{(*Fits of angle distributions} of scattering components*)

Aamode13 $=$ Aaa1 $* \operatorname{Exp}\left[-(x-\text { Aab1 })^{\wedge} 2 /(2 *\right.$ Aac1 $\left.)\right]+$

Aaa2 $* \operatorname{Exp}[-(x-A a b 2) \wedge 2 /(2 *$ Aac2 $)]+$ Aaa $3 * \operatorname{Exp}[-(x-A a b 3) \wedge 2 /(2 *$ Aac3 $)]$;

AAaFitData $=$ FindFit $[\{\text { Angle, AED1 [ [2] }]\}^{\top},\{$ Aamodel3,

$\{\theta \leq \mathrm{Aaa} 1<\operatorname{Max}[\mathrm{ED}], \theta<\mathrm{Aab} 1<70,1<\mathrm{Aac} 1, \theta \leq \mathrm{Aaa} 2<\operatorname{Max}[E D], 30<\mathrm{Aab} 2<80,1<\mathrm{Aac} 2\}$, $0 \leq$ Aaa3 < Max [ED], $30<$ Aab3 < 80, $1<$ Aac3 ,

\{Aaa1, Aab1, Aac1, Aaa2, Aab2, Aac2, Aaa3, Aab3, Aac3\}, X, Maxiterations $\rightarrow$ 500] AaTableFit $=$ Table $[$ Aamodel3 $/$. AAaFitData, $\{x,-40,90\}]$; 


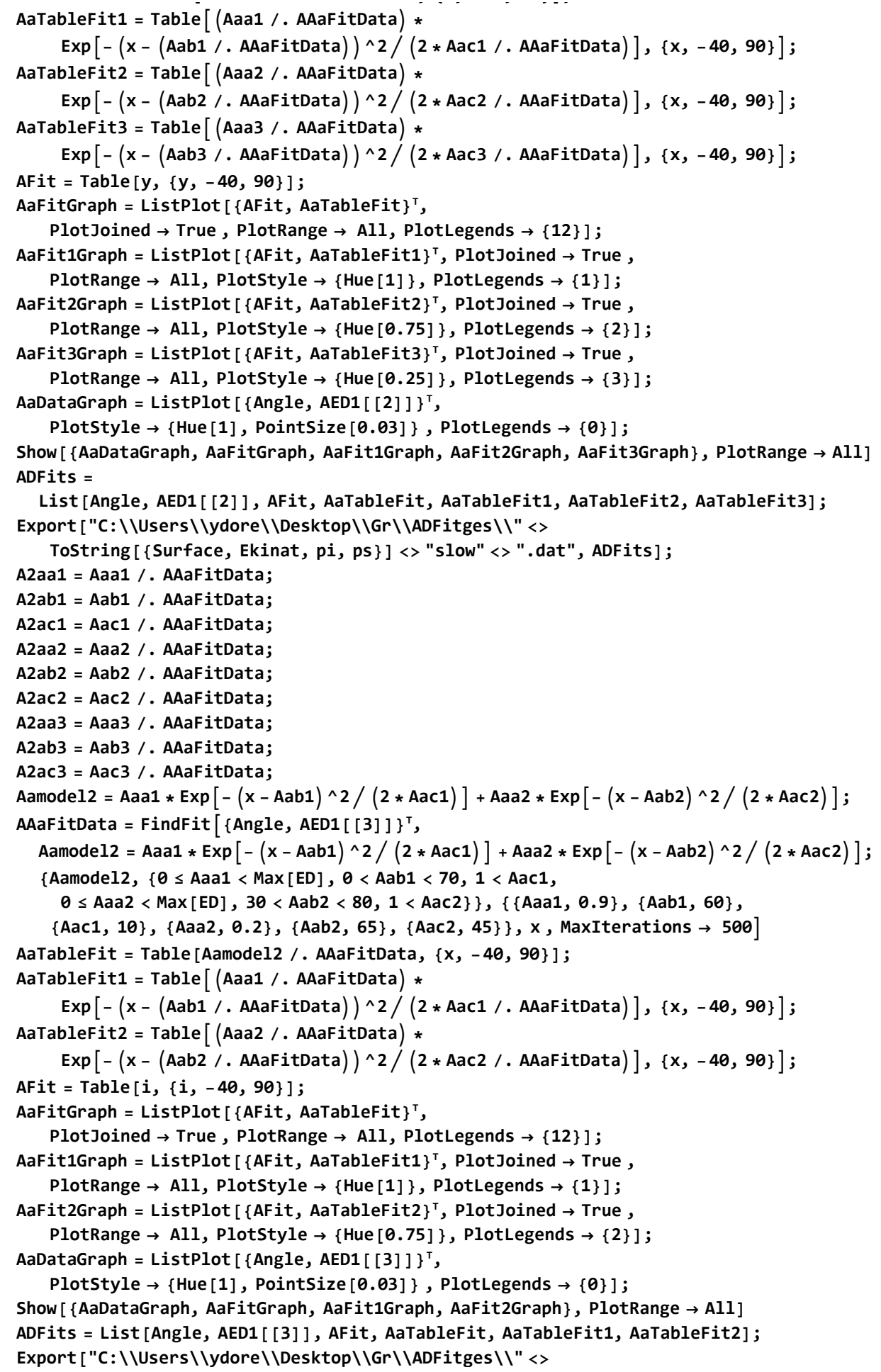


ToString [ $\{$ Surface, Ekinat, pi, ps $\}]<>$ "mid1" <> ".dat", ADFits] ;

A3aa1 = Aaa1 /. AAaFitData;

A3ab1 = Aab1 /. AAaFitData;

A3ac1 = Aac1 /. AAaFitData;

A3aa2 = Aaa2 /. AAaFitData;

A3ab2 = Aab2 /. AAaFitData;

A3ac2 = Aac2 /. AAaFitData;

AAaFitData $=$ FindFit $[\{\text { Angle, AED1 [ [4] }]\}^{\top},\{$ Aamode12, $\{0 \leq$ Aaa1 $<$ Max [ED],

$\theta<$ Aab1 < 70, $1<$ Aac1, $\theta \leq$ Aaa2 < Max [ED], $30<$ Aab2 < 80, $1<$ Aac2 $\}$ \},

$\{\{$ Aaa1, 0.9 $\},\{$ Aab1, 60\}, \{Aac1, 10\}, \{Aaa2, 0.2\}, \{Aab2, 65 $\},\{$ Aac2, 45 $\}$,

$x$, MaxIterations $\rightarrow$ 500]

AaTableFit $=$ Table $[$ Aamodel2 $/$. AAaFitData, $\{x,-40,90\}]$;

AaTableFit1 $=$ Table $[($ Aaa1 $/$. AAaFitData $)$ *

$\operatorname{Exp}\left[-(x-(\text { Aab1 /. AAaFitData }))^{\wedge} 2 /(2 *\right.$ Aac1 /. AAaFitData $\left.\left.)\right],\{x,-40,90\}\right] ;$

AaTableFit2 $=$ Table $[($ Aaa2 $/$. AAaFitData $) *$

$\operatorname{Exp}\left[-(x-(\text { Aab2 /. AAaFitData }))^{\wedge} 2 /(2 *\right.$ Aac2 /. AAaFitData $\left.\left.)\right],\{x,-40,90\}\right] ;$

AFit $=$ Table $[i,\{i,-40,90\}]$;

AaFitGraph $=$ ListPlot $\left[\{\text { AFit, AaTableFit }\}^{\top}\right.$,

PlotJoined $\rightarrow$ True, PlotRange $\rightarrow$ All, PlotLegends $\rightarrow\{12\}]$;

AaFit1Graph $=$ ListPlot $\left[\{\text { AFit, AaTableFit } 1\}^{\top}\right.$, Plot $]$ oined $\rightarrow$ True,

PlotRange $\rightarrow$ All, PlotStyle $\rightarrow$ \{Hue $[1]\}$, PlotLegends $\rightarrow\{1\}]$;

AaFit2Graph $=$ ListPlot $\left[\{\text { AFit, AaTableFit2 }\}^{\top}\right.$, Plot Joined $\rightarrow$ True,

PlotRange $\rightarrow$ All, PlotStyle $\rightarrow$ Hue $[0.75]\}$, PlotLegends $\rightarrow\{2\}]$;

AaDataGraph $=$ ListPlot $[\{$ Angle, AED1 $[4]]\}\}^{\mathrm{T}}$,

PlotStyle $\rightarrow$ \{Hue [1], PointSize $[0.03]\}$, PlotLegends $\rightarrow\{\theta\}]$;

Show [ $\{$ AaDataGraph, AaFitGraph, AaFit1Graph, AaFit2Graph $\}$, PlotRange $\rightarrow$ All]

ADFits = List [Angle, AED1 [ [4] ], AFit, AaTableFit, AaTableFit1, AaTableFit2];

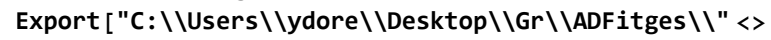

ToString [\{Surface, Ekinat, pi, ps\}] <> "mid2" <> ".dat", ADFits];

A4aa1 = Aaa1 /. AAaFitData;

A4ab1 = Aab1 /. AAaFitData;

A4ac1 = Aac1 /. AAaFitData;

A4aa2 = Aaa2 /. AAaFitData;

A4ab2 = Aab2 /. AAaFitData;

A4ac2 = Aac2 /. AAaFitData;

AAaFitData $=$ FindFit $\left[\{\text { Angle, AED1 }[[5]]\}^{\top},\{\right.$ Aamodel2, $\{\theta \leq$ Aaa1 $<\operatorname{Max}[$ ED $]$,

$\theta<$ Aab1 < 70, $1<$ Aac1, $\theta \leq$ Aaa2 < Max [ED], $30<$ Aab2 < 80, $1<$ Aac2 $\}$ \},

$\{\{$ Aaa1, 0.9 $\},\{$ Aab1, 60\}, \{Aac1, 10\}, \{Aaa2, 0.2\}, \{Aab2, 65 $\},\{$ Aac2, 45$\}\}$,

$X$, MaxIterations $\rightarrow$ 500]

AaTableFit $=$ Table $[$ Aamodel2 $/$. AAaFitData,$\{x,-40,90\}]$;

AaTableFit1 $=$ Table $[($ Aaa1 $/$. AAaFitData $)$ *

$\operatorname{Exp}\left[-(x-(\text { Aab1 /. AAaFitData }))^{\wedge} 2 /(2 *\right.$ Aac1 /. AAaFitData $\left.\left.)\right],\{x,-40,90\}\right] ;$ AaTableFit2 $=$ Table $[($ Aaa2 $/$. AAaFitData $) *$

$\operatorname{Exp}\left[-(x-(\text { Aab2 /. AAaFitData }))^{\wedge} 2 /(2 *\right.$ Aac2 /. AAaFitData $\left.\left.)\right],\{x,-40,90\}\right] ;$

AFit $=$ Table $[i,\{i,-40,90\}]$;

AaFitGraph $=$ ListPlot $\left[\{\text { AFit, AaTableFit }\}^{\top}\right.$,

PlotJoined $\rightarrow$ True, PlotRange $\rightarrow$ All, PlotLegends $\rightarrow\{12\}]$;

AaFit1Graph $=$ ListPlot $\left[\{\text { AFit, AaTableFit1 }\}^{\top}\right.$, Plot]oined $\rightarrow$ True ,

PlotRange $\rightarrow$ All, PlotStyle $\rightarrow$ Hue $[1]\}$, PlotLegends $\rightarrow\{1\}]$;

AaFit2Graph $=$ ListPlot $\left[\{\text { AFit, AaTableFit2 }\}^{\top}\right.$, Plot $]$ oined $\rightarrow$ True,

PlotRange $\rightarrow$ All, PlotStyle $\rightarrow$ \{Hue $[0.75]\}$, PlotLegends $\rightarrow\{2\}]$;

AaDataGraph $=$ ListPlot $[\{\text { Angle, AED1 [ [5] }]\}^{\mathrm{T}}$,

PlotStyle $\rightarrow\{$ Hue $[1]$, PointSize $[0.03]\}$, PlotLegends $\rightarrow\{\theta\}]$;

Show [ $\{$ AaDataGraph, AaFitGraph, AaFit1Graph, AaFit2Graph $\}$, PlotRange $\rightarrow$ All]

ADFits = List [Angle, AED1 [5] ], AFit, AaTableFit, AaTableFit1, AaTableFit2]; 


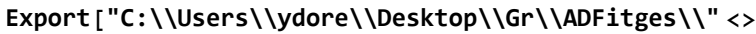

ToString [ $\{$ Surface, Ekinat, pi, ps\}] <> "fast" <> ".dat", ADFits];

A5aa1 = Aaa1 /. AAaFitData;

A5ab1 = Aab1 /. AAaFitData;

A5ac1 = Aac1 /. AAaFitData;

A5aa2 = Aaa2 /. AAaFitData;

A5ab2 = Aab2 /. AAaFitData;

A5ac2 = Aac2 /. AAaFitData;

Fit3D1 = Integrate $\left[\right.$ fea1 $* \operatorname{Exp}\left[-(x-\text { feb1 })^{\wedge} 2 /(2 *\right.$ fec1 $\left.)\right]$ *

$\left(\operatorname{Exp}[-(y) \wedge 2 /(2 * A 2 a c 1)]+\operatorname{Exp}\left[-(y)^{\wedge} 2 /(2 * A 2 a c 2)\right]+\operatorname{Exp}\left[-(y)^{\wedge} 2 /(2 * A 2 a c 3)\right]\right)$,

$\{x, 0,1.2\},\{y,-90,90\}]$;

Fit3D2 = Integrate $\left[\mathrm{fea} 2 * \operatorname{Exp}\left[-(x-\mathrm{feb} 2)^{\wedge} 2 /(2 * \mathrm{fec} 2)\right]\right.$ *

$\left.\left(\operatorname{Exp}\left[-(y)^{\wedge} 2 /(2 * A 3 a c 1)\right]+\operatorname{Exp}\left[-(y)^{\wedge} 2 /(2 * A 3 a c 2)\right]\right),\{x, 0,1.2\},\{y,-90,90\}\right] ;$

Fit3D3 = Integrate $[$ fea $3 * \operatorname{Exp}[-(x-\mathrm{feb} 3) \wedge 2 /(2 *$ fec 3$)] *$

$\left.\left(\operatorname{Exp}\left[-(y)^{\wedge} 2 /(2 * A 4 a c 1)\right]+\operatorname{Exp}\left[-(y)^{\wedge} 2 /(2 * A 4 a c 2)\right]\right),\{x, 0,1.2\},\{y,-90,90\}\right]$

Fit3D4 = Integrate $\left[\right.$ fea4 $* \operatorname{Exp}\left[-(x-\text { feb4 })^{\wedge} 2 /(2 *\right.$ fec4 $\left.)\right]$ *

$\left.\left(\operatorname{Exp}\left[-(y)^{\wedge} 2 /(2 * A 5 a c 1)\right]+\operatorname{Exp}\left[-(y)^{\wedge} 2 /(2 * A 5 a c 2)\right]\right),\{x, 0,1.2\},\{y,-90,90\}\right] ;$

IntList3D $=\{$ List $[\operatorname{Cos}[\mathrm{pr} / 180 * \pi] *$ Eingabe $[[i, 2]]$,

$\operatorname{Cos}[\mathrm{pc} / 180 * \pi] *$ Eingabe $[i, 2]]$, Fit3D1, Fit3D2 + Fit3D3, Fit3D4] $\}$

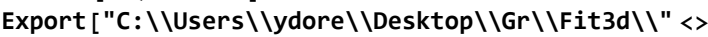

ToString [ Surface, Ekinat, pi, ps\}] <>".dat", IntList3D] ;

$\{$ Aaa $1 \rightarrow 0.224963$, Aab1 $\rightarrow$ 10.0915, Aac1 $\rightarrow$ 493.979, Aaa2 $\rightarrow 0.196598$,

Aab2 $\rightarrow 62.5066$, Aac2 $\rightarrow 97.5895$, Aaa3 $\rightarrow 0.510019$, Aab3 $\rightarrow 37.6219$, Aac3 $\rightarrow 162.173\}$

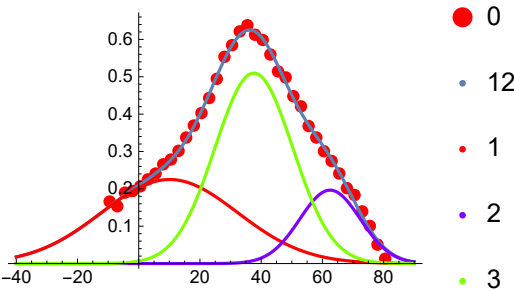

Aaa1 $\rightarrow 0.237208$, Aab1 $\rightarrow 54.5213$, Aac1 $\rightarrow 186.589$,

Aaa2 $\rightarrow 0.111589$, Aab2 $\rightarrow 55.1218$, Aac $2 \rightarrow 16.4608$

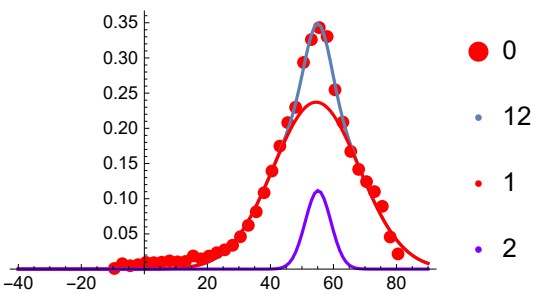

$\{$ Aaa1 $\rightarrow 0.0900004$, Aab1 $\rightarrow 51.5857$, Aac1 $\rightarrow 60.8068$

Aaa2 $\rightarrow 0.0507225$, Aab2 $\rightarrow 71.0207$, Aac2 $\rightarrow 32.2246\}$ 
10 | FitGr1_Nifirstappendix.nb

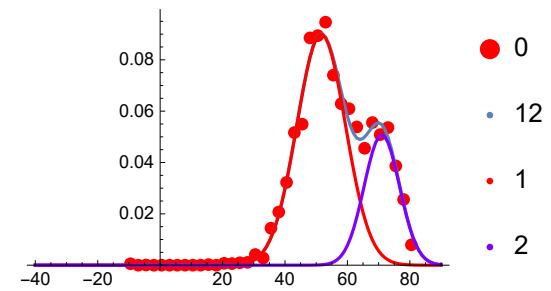

$\{$ Aaa1 $\rightarrow 0.0638224$, Aab1 $\rightarrow 51.3135$, Aac1 $\rightarrow 29.8921$,

Aaa2 $\rightarrow 0.0373704$, Aab2 $\rightarrow 70.3044$, Aac2 $\rightarrow 47.8122\}$

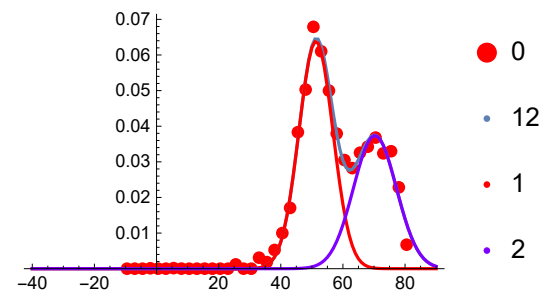

$\{\{1.18207,1.30944,2127.13,483.345,45.0784223408877\}\}$ 



\section{Bibliography}

[1] G. Ertl, "Elementary Steps in Heterogeneous Catalysis", Angew. Chem. Int. Ed. 1990, 29, 1219-1227.

[2] U. Nations, World Population Prospects: The 2017 Revision, Key Findings and Advance Tables. Report, Working Paper No. ESA/P/WP/248, 2017.

[3] H. T. Hwang, A. Varma, "Hydrogen storage for fuel cell vehicles", Curr. Opinion Chem. Eng. 2014, 5, 42-48.

[4] K. W. Kolasinski, Surface Science: Foundations of Catalysis and Nanoscience, John Wiley and Sons, Ltd, UK, 2012.

[5] N. Media, Nobel Prizes, Report, 2018.

[6] A. C. Luntz, The Dynamics of Making and Breaking Bonds at Surfaces, Elsevier, Amsterdam, 2008, pp. 143-254.

[7] K. Golibrzuch, N. Bartels, D. J. Auerbach, A. M. Wodtke, "The dynamics of molecular interactions and chemical reactions at metal surfaces: testing the foundations of theory", Annu. Rev. Phys. Chem. 2015, 66, 399-425.

[8] A. M. Schwartzberg, D. Olynick, "Complex Materials by Atomic Layer Deposition", Adv. Mater. 2015, 27, 5778-84.

[9] R. M. Costescu, D. G. Cahill, F. H. Fabreguette, Z. A. Sechrist, S. M. George, "Ultra-low thermal conductivity in W/Al2O3 nanolaminates", Science 2004, 303, 989-990.

[10] F. Döring, A. Major, C. Eberl, H.-U. Krebs, "Minimized thermal conductivity in highly stable thermal barrier $\mathrm{W} / \mathrm{ZrO} 2$ multilayers", Appl. Phys. A 2016, 122, 872.

[11] S.-J. Ding, H. Hu, C. Zhu, M. F. Li, S. J. Kim, B. J. Cho, D. S. H. Chan, M. B. $\mathrm{Yu}, \mathrm{A}$. Y. Du, A. Chin, D.-L. Kwong, "Evidence and Understanding of ALD HfO2-Al2O3 Laminate MIM Capacitors Outperforming Sandwich Counterparts", IEEE Electron Device Lett. 2004, 25, 681-683. 
[12] I.-S. Park, K.-m. Ryu, J. Jeong, J. Ahn, "Dielectric Stacking Effect of Al2O3 and HfO2 in Metal-Insulator-Metal Capacitor", IEEE Electron Device Lett. 2013, 34, $120-122$.

[13] S. Y. Zhou, G. H. Gweon, A. V. Fedorov, P. N. First, W. A. de Heer, D. H. Lee, F. Guinea, A. H. Castro Neto, A. Lanzara, "Substrate-induced bandgap opening in epitaxial graphene", Nat. Mater. 2007, 6, 770.

[14] C. Lee, X. Wei, J. W. Kysar, J. Hone, "Measurement of the Elastic Properties and Intrinsic Strength of Monolayer Graphene", Science 2008, 321, 385-388.

[15] D. Hollenbach, E. E. Salpeter, "Surface Recombination of Hydrogen Molecules", Astrophys. J. 1971, 163, 155-164.

[16] G. Vidali, J. E. Roser, G. Manicó, V. Pirronello, "A Summary of Experimental Results on Molecular Hydrogen Formation on Dust Grain Analogues", Proc. Int. Astron. Union 2006, 1, 355-364.

[17] L. Schlapbach, A. Züttel, "Hydrogen-storage materials for mobile applications", Nature 2001, 414, 353.

[18] D. C. Elias, R. R. Nair, T. M. G. Mohiuddin, S. V. Morozov, P. Blake, M. P. Halsall, A. C. Ferrari, D. W. Boukhvalov, M. I. Katsnelson, A. K. Geim, K. S. Novoselov, "Control of Graphene's Properties by Reversible Hydrogenation: Evidence for Graphane", Science 2009, 323, 610-613.

[19] S. E. Huber, T. Hell, M. Probst, A. Ostermann, "Numerical investigation of the elastic scattering of hydrogen (isotopes) and helium at graphite (0001) surfaces at beam energies of 1 to $4 \mathrm{eV}$ using a split-step Fourier method", Theor. Chem. Acc. 2013, 132, 1337.

[20] R. Grassi, T. Low, M. Lundstrom, "Scaling of the Energy Gap in PatternHydrogenated Graphene", Nano Lett. 2011, 11, 4574-4578.

[21] D. Haberer, D. V. Vyalikh, S. Taioli, B. Dora, M. Farjam, J. Fink, D. Marchenko, T. Pichler, K. Ziegler, S. Simonucci, M. S. Dresselhaus, M. Knupfer, B. Büchner, A. Grüneis, "Tunable Band Gap in Hydrogenated Quasi-Free-Standing Graphene", Nano Lett. 2010, 10, 3360-3366.

[22] C. Lin, Y. Feng, Y. Xiao, M. Dür, X. Huang, X. Xu, R. Zhao, E. Wang, X.-Z. Li, Z. Hu, "Direct Observation of Ordered Configurations of Hydrogen Adatoms on Graphene", Nano Lett. 2015, 15, 903-908. 
[23] K. S. Novoselov, A. K. Geim, S. V. Morozov, D. Jiang, Y. Zhang, S. V. Dubonos, I. V. Grigorieva, A. A. Firsov, "Electric Field Effect in Atomically Thin Carbon Films", Science 2004, 306, 666-669.

[24] M. P. Andersson, F. Abild-Pedersen, I. N. Remediakis, T. Bligaard, G. Jones, J. Engbaek, O. Lytken, S. Horch, J. H. Nielsen, J. Sehested, J. R. Rostrup-Nielsen, J. K. Norskov, I. Chorkendorff, "Structure sensitivity of the methanation reaction: H2-induced CO dissociation on nickel surfaces", J. Catal. 2008, 255, 6-19.

[25] D. Hollenbach, E. E. Salpeter, "Surface Adsorption of Light Gas Atoms", J. Chem. Phys. 1970, 53, 79-86.

[26] Z. Medina, B. Jackson, "Quantum studies of light particle trapping, sticking, and desorption on metal and graphite surfaces", J Chem Phys 2008, 128, 114704.

[27] G. J. Kroes, M. Pavanello, M. Blanco-Rey, M. Alducin, D. J. Auerbach, "Ab initio molecular dynamics calculations on scattering of hyperthermal $\mathrm{H}$ atoms from $\mathrm{Cu}(111)$ and $\mathrm{Au}(111) "$, J. Chem. Phys. 2014, 141, 054705.

[28] O. Bünermann, H. Jiang, Y. Dorenkamp, A. Kandratsenka, S. M. Janke, D. J. Auerbach, A. M. Wodtke, "Electron-hole pair excitation determines the mechanism of hydrogen atom adsorption", Science 2015, 350, 1346-1349.

[29] H. Jiang, Dynamics of Hydrogen Atoms Scattering from Surfaces, Thesis, GeorgAugust-University Göttingen, 2016.

[30] J. E. Hurst, L. Wharton, K. C. Janda, D. J. Auerbach, "Trapping-desorption scattering of argon from Pt(111)", J. Chem. Phys. 1985, 83, 1376-1381.

[31] A. W. Kleyn, Basic Mechanisms in Atom-Surface Interactions, North-Holland, 2008, pp. 29-52.

[32] M. Born, R. Oppenheimer, "Zur Quantentheorie der Molekeln", Ann. Phys. 1927, $84,457-484$.

[33] J. C. Tully, "Perspective on Zur Quantentheorie der Molokeln", Theor. Chem. Acc. 2000, 103, 173-176.

[34] E. K. Grimmelmann, J. C. Tully, M. J. Cardillo, "Hard-cube model analysis of gas-surface energy accommodation", J. Chem. Phys. 1980, 72, 1039-1043.

[35] J. E. Hurst, L. Wharton, K. C. Janda, D. J. Auerbach, "Direct inelastic scattering Ar from Pt(111)", J. Chem. Phys. 1983, 78, 1559-1581.

[36] R. J.W. E. Lahaye, A. W. Kleyn, S. Stolte, S. Holloway, "The scattering of Ar from $\operatorname{Ag}(111)$ : a molecular dynamics study", Surf. Sci. 1995, 338, 169-182. 
[37] D. Kulginov, M. Persson, C. T. Rettner, D. S. Bethune, "An Empirical Interaction Potential for the Ar/Pt(111) System", J. Phys. Chem. 1996, 100, 7919-7927.

[38] J. C. Tully, "Washboard model of gas-surface scattering", J. Chem. Phys. 1990, 92, 680-686.

[39] A. Groß, Theoretical Surface Science, Springer Verlag, Berlin Heidelberg, 2009.

[40] P. Nieto, E. Pijper, D. Barredo, G. Laurent, R. A. Olsen, E.-J. Baerends, G.-J. Kroes, D. Farías, "Reactive and Nonreactive Scattering of H2 from a Metal Surface Is Electronically Adiabatic", Science 2006, 312, 86-89.

[41] A. Groß, "Reactions at surfaces studied by ab initio dynamics calculations", Surf. Sci. Rep. 1998, 32, 291-340.

[42] A. M. Wodtke, J. C. Tully, D. J. Auerbach, "Electronically non-adiabatic interactions of molecules at metal surfaces: Can we trust the Born-Oppenheimer approximation for surface chemistry?", Int. Rev. Phys. Chem. 2004, 23, 513-539.

[43] A. M. Wodtke, D. Matsiev, D. J. Auerbach, "Energy transfer and chemical dynamics at solid surfaces: The special role of charge transfer", Progr. Surf. Sci. 2008, 83, $167-214$.

[44] H. Nienhaus, "Electronic excitations by chemical reactions on metal surfaces", Surf. Sci. Rep. 2002, 45, 1-78.

[45] H. Nienhaus, H. S. Bergh, B. Gergen, A. Majumdar, W. H. Weinberg, E. W. McFarland, "Direct detection of electron-hole pairs generated by chemical reactions on metal surfaces", Surf. Sci. 2000, 445, 335-342.

[46] H. Nienhaus, H. S. Bergh, B. Gergen, A. Majumdar, W. H. Weinberg, E. W. McFarland, "Electron-Hole Pair Creation at Ag and Cu Surfaces by Adsorption of Atomic Hydrogen and Deuterium", Phys. Rev. Lett. 1999, 82, 446-449.

[47] B. Gergen, H. Nienhaus, W. H. Weinberg, E. W. McFarland, "Chemically Induced Electronic Excitations at Metal Surfaces", Science 2001, 294, 2521-2523.

[48] B. Gergen, S. J. Weyers, H. Nienhaus, W. H. Weinberg, E. W. McFarland, "Observation of excited electrons from nonadiabatic molecular reactions of NO and O2 on polycrystalline Ag", Surf. Sci. 2001, 488, 123-132.

[49] E. Hasselbrink, "How non-adiabatic are surface dynamical processes?", Curr. Opinion Solid State Mater. Sci. 2006, 10, 192-204.

[50] J. R. Trail, D. M. Bird, M. Persson, S. Holloway, "Electron-hole pair creation by atoms incident on a metal surface", J. Chem. Phys. 2003, 119, 4539-4549. 
[51] S. M. Janke, D. J. Auerbach, A. M. Wodtke, A. Kandratsenka, "An accurate fulldimensional potential energy surface for $\mathrm{H}-\mathrm{Au}(111)$ : Importance of nonadiabatic electronic excitation in energy transfer and adsorption", J. Chem. Phys. 2015, 143, 124708 .

[52] A. Kandratsenka, H. Jiang, Y. Dorenkamp, S. M. Janke, M. Kammler, A. M. Wodtke, O. Bünermann, "Unified description of H-atom-induced chemicurrents and inelastic scattering", PNAS 2018, 115, 680-684.

[53] J. V. Barth, H. Brune, G. Ertl, R. J. Behm, "Scanning tunneling microscopy observations on the reconstructed $\mathrm{Au}(111)$ surface: Atomic structure, long-range superstructure, rotational domains, and surface defects", Phys. Rev. B 1990, 42, 9307-9318.

[54] K. Hermann, F. Rammer, "SURFACE EXPLORER software based on BALSAC", Software 2017, http://surfexp.fhi-berlin.mpg.de/.

[55] U. Harten, A. M. Lahee, J. Peter Toennies, C. Woll, "Observation of a soliton reconstruction of $\mathrm{Au}(111)$ by high-resolution helium-atom diffraction", Phys. Rev. Lett. 1985, 54, 2619-2622.

[56] F. Hanke, J. Björk, "Structure and local reactivity of the $\mathrm{Au}(111)$ surface reconstruction", Phys. Rev. B 2013, 87, 235422.

[57] J. Rumble, CRC Handbook of chemistry and Physics, 98th Edition, CRC Press, 2017.

[58] D. A. Papaconstantopoulos, Handbook of the Band Structure of Elemental Solids, Springer, New York, 2015.

[59] S. D. Kevan, R. H. Gaylord, "High-resolution photoemission study of the electronic structure of the noble-metal(111) surfaces", Phys. Rev. B 1987, 36, 5809-5818.

[60] P. Roos, E. Bertel, K. D. Rendulic, "Observation of an sp-derived surface resonance on $\mathrm{Pt}(111)$ indicating the crucial role of surface states in physisorption", Chem. Phys. Lett. 1995, 232, 537-541.

[61] R. H. French, "Electronic Band Structure of Al2O3, with Comparison to Alon and AIN", J. Am. Ceram. Soc. 1990, 73, 477-489.

[62] L. Zhang, H. C. Jiang, C. Liu, J. W. Dong, P. Chow, "Annealing of Al2O3 thin films prepared by atomic layer deposition", J. Phys. D: Appl. Phys. 2007, 40, $3707-3713$. 
[63] J. Ahn, J. W. Rabalais, "Composition and structure of the Al2O3(0001)-(1 x 1) surface", Surf. Sci. 1997, 388, 121-131.

[64] E. A. Soares, M. A. Van Hove, C. F. Walters, K. F. McCarty, "Structure of the $\alpha-\mathrm{Al}_{2} \mathrm{O}_{3}(0001)$ surface from low-energy electron diffraction: $\mathrm{Al}$ termination and evidence for anomalously large thermal vibrations", Phys. Rev. B 2002, 65, 195405.

[65] J. Guo, D. E. Ellis, D. J. Lam, "Electronic structure and energetics of sapphire (0001) and (1102) surfaces", Phys. Rev. B 1992, 45, 13647-13656.

[66] T. Suzuki, S. Hishita, K. Oyoshi, R. Souda, "Structure of $\alpha$-Al2O3(0001) surface and Ti deposited on $\alpha$-Al2O3(0001) substrate: CAICISS and RHEED study", Surf. Sci. 1999, 437, 289-298.

[67] P. J. Eng, T. P. Trainor, G. E. Brown Jr., G. A. Waychunas, M. Newville, S. R. Sutton, M. L. Rivers, "Structure of the Hydrated $\alpha$-Al2O3 (0001) Surface", Science 2000, 288, 1029-1033.

[68] A. H. Castro Neto, F. Guinea, N. M. R. Peres, K. S. Novoselov, A. K. Geim, "The electronic properties of graphene", Rev. Mod. Phys. 2009, 81, 109-162.

[69] D. R. Cooper, B. D'Anjou, N. Ghattamaneni, B. Harack, M. Hilke, A. Horth, N. Majlis, M. Massicotte, L. Vandsburger, E. Whiteway, V. Yu, "Experimental Review of Graphene", ISRN Condensed Matter Physics 2012, 2012, 1-56.

[70] E. Voloshina, Y. Dedkov, "Graphene on metallic surfaces: problems and perspectives", Phys. Chem. Chem. Phys. 2012, 14, 13502-13514.

[71] L. Wirtz, A. Rubio, "The phonon dispersion of graphite revisited", Solid State Commun. 2004, 131, 141-152.

[72] J. Wintterlin, M. L. Bocquet, "Graphene on metal surfaces", Surf. Sci. 2009, 603, $1841-1852$.

[73] W. B. Zhang, C. Chen, P. Y. Tang, "First-principles study for stability and binding mechanism of graphene/Ni(111) interface: Role of vdW interaction", $J$ Chem Phys 2014, 141, 044708.

[74] Z. Fogarassy, M. H. Rümmeli, S. Gorantla, A. Bachmatiuk, G. Dobrik, K. Kamarás, L. P. Biró, K. Havancsák, J. L. Lábár, "Dominantly epitaxial growth of graphene on Ni (111) substrate", Appl. Surf. Sci. 2014, 314, 490-499.

[75] Y. Gamo, A. Nagashima, M. Wakabayashi, M. Terai, C. Oshima, "Atomic structure of monolayer graphite formed on Ni(111)", Surf. Sci. 1997, 374, 61-64. 
[76] D. E. Parreiras, E. A. Soares, G. J. P. Abreu, T. E. P. Bueno, W. P. Fernandes, V. E. de Carvalho, S. S. Carara, H. Chacham, R. Paniago, "Graphene/Ni(111) surface structure probed by low-energy electron diffraction, photoelectron diffraction, and first-principles calculations", Phys. Rev. B 2014, 90, 155454.

[77] S. M. Kozlov, F. Viñes, A. Görling, "Bonding Mechanisms of Graphene on Metal Surfaces", J. Phys. Chem. C 2012, 116, 7360-7366.

[78] F. Bianchini, L. L. Patera, M. Peressi, C. Africh, G. Comelli, "Atomic Scale Identification of Coexisting Graphene Structures on Ni(111)", J. Phys. Chem. Lett. 2014, 5, 467-473.

[79] K. Hitoshi, O. Hidenori, Y. Takashige, G. Yoshihiko, S. Ryutaro, "Structure Analysis of Monolayer Graphite on $\mathrm{Ni}(111)$ Surface by Li+ - Impact Collision Ion Scattering Spectroscopy", Jpn. J. Appl. Phys. 2002, 41, 6149.

[80] G. Bertoni, L. Calmels, A. Altibelli, V. Serin, "First-principles calculation of the electronic structure and EELS spectra at the graphene/Ni(111) interface", Phys. Rev. B 2005, 71, 075402.

[81] A. Tamtögl, E. Bahn, J. Zhu, P. Fouquet, J. Ellis, W. Allison, "Graphene on $\mathrm{Ni}(111)$ : Electronic Corrugation and Dynamics from Helium Atom Scattering", $J$. Phys. Chem. C 2015, 119, 25983-25990.

[82] F. Mittendorfer, A. Garhofer, J. Redinger, J. Klimeš, J. Harl, G. Kresse, "Graphene on $\mathrm{Ni}(111)$ : Strong interaction and weak adsorption", Phys. Rev. B 2011, 84 , 201401.

[83] A. Nagashima, N. Tejima, C. Oshima, "Electronic states of the pristine and alkalimetal-intercalated monolayer graphite/Ni(111) systems", Phys. Rev. B 1994, 50, 17487-17495.

[84] T. Aizawa, R. Souda, Y. Ishizawa, H. Hirano, T. Yamada, K.-i. Tanaka, C. Oshima, "Phonon dispersion in monolayer graphite formed on $\mathrm{Ni}(111)$ and $\mathrm{Ni}(001)$ ", Surf. Sci. 1990, 237, 194-202.

[85] A. Dahal, M. Batzill, "Graphene-nickel interfaces: a review", Nanoscale 2014, 6, $2548-62$.

[86] G. Odahara, S. Otani, C. Oshima, M. Suzuki, T. Yasue, T. Koshikawa, "In-situ observation of graphene growth on Ni(111)", Surf. Sci. 2011, 605, 1095-1098.

[87] R. Addou, A. Dahal, P. Sutter, M. Batzill, "Monolayer graphene growth on Ni(111) by low temperature chemical vapor deposition", Appl. Phys. Lett. 2012, 100, 021601. 
[88] J. Lahiri, T. S Miller, A. J Ross, L. Adamska, I. I. Oleynik, M. Batzill, "Graphene growth and stability at nickel surfaces", New J. Phys. 2011, 13, 025001.

[89] L. L. Patera, C. Africh, R. S. Weatherup, R. Blume, S. Bhardwaj, C. CastellarinCudia, A. Knop-Gericke, R. Schloegl, G. Comelli, S. Hofmann, C. Cepek, "In Situ Observations of the Atomistic Mechanisms of Ni Catalyzed Low Temperature Graphene Growth", ACS Nano 2013, 7, 7901-7912.

[90] L. Schnieder, K. Seekamp-Rahn, F. Liedeker, H. Steuwe, K. H. Welge, "Hydrogen exchange reaction H + D2 in crossed beams", Faraday Discuss. Chem. Soc. 1991, 91, 259-269.

[91] A. B. Alekseyev, H.-P. Liebermann, D. B. Kokh, R. J. Buenker, "On the ultraviolet photofragmentation of hydrogen iodide", J. Chem. Phys. 2000, 113, 6174-6185.

[92] G. Scoles, Atomic and Molecular Beam Methods, Vol. 1, Oxford University Press, New York, Oxford, 1988.

[93] S. R. Langford, P. M. Regan, A. J. Orr-Ewing, M. N. R. Ashfold, "On the UV photodissociation dynamics of hydrogen iodide", Chem. Phys. 1998, 231, 245-260.

[94] P. M. Regan, S. R. Langford, A. J. Orr-Ewing, M. N. R. Ashfold, "The ultraviolet photodissociation dynamics of hydrogen bromide", J. Chem. Phys. 1999, 110, 281.

[95] R. E. Smalley, L. Wharton, D. H. Levy, "Molecular optical spectroscopy with supersonic beams and jets", Acc. Chem. Res. 1977, 10, 139-145.

[96] J. Libuda, H. J. Freund, "Molecular beam experiments on model catalysts", Surf. Sci. Rep. 2005, 57, 157-298.

[97] K. Huber, G. Herzberg, Molecular Spectra and Molecular Structure, Van Nostrand Reinhold Company, New York, 1979.

[98] B. d. Darwent, "Bond Dissociation Energies in Simple Molecules", NSRDS-NBS $1970,31$.

[99] M. N. R. Ashfold, D. H. Mordaunt, S. H. S. Wilson in Advances in Photochemistry, John Wiley and Sons, Inc., 2007, pp. 217-295.

[100] W. Demtröder, Experimentalphysik 3: Atome, Moleküle und Festkörper, Springer Verlag, Berlin Heidelberg, 2010.

[101] W. A. Chupka, "Factors affecting lifetimes and resolution of Rydberg states observed in zero-electron-kinetic-energy spectroscopy", J. Chem. Phys. 1993, 98, $4520-4530$. 
[102] A. ten Wolde, L. D. Noordam, A. Lagendijk, H. B. van Linden van den Heuvell, "Atomic electron wave packets in an electrical field", Phys. Rev. A 1989, 40, 485488.

[103] X. Yang, "State-to-state dynamics of elementary chemical reactions using Rydberg H-atom translational spectroscopy", Int. Rev. Phys. Chem. 2005, 24, 37-98.

[104] G. Hilber, A. Lago, R. Wallenstein, "Broadly tunable vacuum-ultraviolet/extremeultraviolet radiation generated by resonant third-order frequency conversion in krypton", J. Opt. Soc. Am. B 1987, 4, 1753-1764.

[105] K. Yuan, L. Cheng, Y. Cheng, Q. Guo, D. Dai, X. Yang, "Tunable VUV photochemistry using Rydberg H-atom time-of-flight spectroscopy", Rev. Sci. Instrum. 2008, 79, 124101.

[106] R. Hilbig, R. Wallenstein, "Enhanced production of tunable VUV radiation by phase-matched frequency tripling in krypton and xenon", IEEE J. Quantum Electron. 1981, 17, 1566-1573.

[107] J. P. Marangos, N. Shen, H. Ma, M. H. R. Hutchinson, J. P. Connerade, "Broadly tunable vacuum-ultraviolet radiation source employing resonant enhanced sumdifference frequency mixing in krypton", J. Opt. Soc. Am. B 1990, 7, 1254-1263.

[108] L. Meitner, "Über die beta-Strahl-Spektra und ihren Zusammenhang mit der gamma-Strahlung", Z. Phys. 1922, 11, 35-54.

[109] P. Auger, "Sur l'effet photoélectrique composé", J. de Physique et le Radium 1925, 6, 205-208.

[110] L. E. Davis, N. C. MacDonald, P. W. Palmberg, G. E. Riach, R. E. Weber, Handbook of Auger Electron Spectroscopy, Physical Electronics Industries, Inc., Minnesota, 1976.

[111] K. Pussi, R. D. Diehl, Low Energy Electron Diffraction, John Wiley and Sons, Inc., 2002.

[112] A. Patane, N. Balkan, Semiconductor Research: Experimental Techniques, SpringerVerlag, Berlin Heidelberg, 2012.

[113] P. R. Norton, J. A. Davies, T. E. Jackman, "Absolute coverages of CO and O on Pt(111); Comparison of saturation CO coverages on Pt(100), (110) and (111) surfaces", Surf. Sci. 1982, 122, L593-L600.

[114] H. Steininger, S. Lehwald, H. Ibach, "Adsorption of oxygen on Pt(111)", Surf. Sci. 1982, 123, 1-17. 
[115] R. G. Musket, W. McLean, C. A. Colmenares, D. M. Makowiecki, W. J. Siekhaus, "Preparation of atomically clean surfaces of selected elements: A Review", Appl. Surf. Sci. 1982, 10, 143-207.

[116] W. Zhao, J. Gebhardt, F. Spath, K. Gotterbarm, C. Gleichweit, H. P. Steinruck, A. Gorling, C. Papp, "Reversible hydrogenation of graphene on $\mathrm{Ni}(111)$-synthesis of graphone", Chemistry 2015, 21, 3347-58.

[117] Y. Dorenkamp, C. Volkmann, V. Roddatis, S. Schneider, A. M. Wodtke, O. Bünermann, "Inelastic H Atom Scattering from Ultrathin Aluminum Oxide Films Grown by Atomic Layer Deposition on Pt(111)", J. Phys. Chem. C 2018, 122, 10096-10102.

[118] S. M. Georg, A. W. Ott, J. W. Klaus, "Surface Chemistry for Atomic Layer Growth", J. Phys. Chem. 1996, 100, 13121-13131.

[119] Z. A. Sechrist, F. H. Fabreguette, O. Heintz, T. M. Phung, D. C. Johnson, S. M. George, "Optimization and Structural Characterization of W/Al2O3 Nanolaminates Grown Using Atomic Layer Deposition Techniques", Chem. Mater. 2005, $17,3475-3485$.

[120] B. C. Kruger, S. Meyer, A. Kandratsenka, A. M. Wodtke, T. Schafer, "Vibrational Inelasticity of Highly Vibrationally Excited $\mathrm{NO}$ on $\mathrm{Ag}(111)$ ", J. Phys. Chem. Lett. 2016, $7,441-446$.

[121] Y. Huang, C. T. Rettner, D. J. Auerbach, A. M. Wodtke, "Vibrational Promotion of Electron Transfer", Science 2000, 290, 111-114.

[122] B. Mildner, E. Hasselbrink, D. Diesing, "Electronic excitations induced by surface reactions of H and D on gold", Chem. Phys. Lett. 2006, 432, 133-138.

[123] B. Schindler, D. Diesing, E. Hasselbrink, "Electronic Excitations in the Course of the Reaction of $\mathrm{H}$ with Coinage and Noble Metal Surfaces: A Comparison", Z. Phys. Chem. 2013, 227, 1381-1395.

[124] D. P. Engelhart, R. J. V. Wagner, A. Meling, A. M. Wodtke, T. Schäfer, "Temperature programmed desorption of weakly bound adsorbates on $\mathrm{Au}(111)$ ", Surf. Sci. 2016, 650, 11-16.

[125] J. Strömquist, L. Bengtsson, M. Persson, B. Hammer, "The dynamics of H absorption in and adsorption on $\mathrm{Cu}(111)$ ", Surf. Sci. 1998, 397, 382-394.

[126] S. M. Janke, A. Kandratsenka, "personal communication". 
[127] Y. Dorenkamp, H. Jiang, H. Köckert, N. Hertl, M. Kammler, S. M. Janke, A. Kandratsenka, A. M. Wodtke, O. Bünermann, "Hydrogen collisions with transition metal surfaces: Universal electronically nonadiabatic adsorption", J. Chem. Phys. 2018, 148, 034706.

[128] S. M. Janke, Theoretical Description off Hydrogen Atom Scattering of Noble Metals, Thesis, Georg-August University Göttingen, 2016.

[129] M. Kammler, S. M. Janke, A. Kandratsenka, A. M. Wodtke, "Genetic algorithm approach to global optimization of the full-dimensional potential energy surface for hydrogen atom at fcc-metal surfaces", Chem. Phys. Lett. 2017, 683, 286-290.

[130] J. Gottschalck, B. Hammer, "A density functional theory study of the adsorption of sulfur, mercapto, and methylthiolate on $\mathrm{Au}(111)$ ", J. Chem. Phys. 2002, 116, $784-790$.

[131] A. Montoya, A. Schlunke, B. S. Haynes, "Reaction of Hydrogen with Ag(111): Binding States, Minimum Energy Paths, and Kinetics", J. Phys. Chem. B 2006, 110, 17145-17154.

[132] Y. Liu, Y. Huang, Z. Xiao, X. Reng, "Study of Adsorption of Hydrogen on Al, Cu, Mg, Ti Surfaces in Al Alloy Melt via First Principles Calculation", Metals 2017, $7,21$.

[133] J. A. Keith, G. Jerkiewicz, T. Jacob, "Theoretical investigations of the oxygen reduction reaction on $\mathrm{Pt}(111)$ ", Chem. Phys. Chem. 2010, 11, 2779-94.

[134] M. S. Daw, S. M. Foiles, "Theory of subsurface occupation, ordered structures, and order-disorder transitions for hydrogen on $\mathrm{Pd}(111)$ ", Phys. Rev. B 1987, 35, $2128-2136$.

[135] J. K. Nørskov, "Calculated Binding Properties of Hydrogen on Nickel Surfaces", Phys. Rev. Lett. 1982, 48, 1620-1624.

[136] J. E. Angelo, N. R. Moody, M. I. Baskes, "Trapping of hydrogen to lattice defects in nickel", Modelling Simul. Mater. Sci. Eng. 1995, 3, 289-307.

[137] Y. Li, G. Wahnström, "Molecular-dynamics simulation of hydrogen diffusion in palladium", Phys. Rev. B 1992, 46, 14528-14542.

[138] R. Ohrlich, W. Drexel in 4th IAEA Symp. Neutron Inelastic Scattering, Vol. 1, 4th IAEA Symp. Neutron Inelastic Scattering, p. 208. 
[139] V. A. Korshunov, "Determination of the phonon density of states from the thermodynamic functions of a crystal: Nickel, palladium, and platinum", Sov. Phys. J. 1979, 22, 903-905.

[140] Y. Wang, J. J. Wang, W. Y. Wang, Z. G. Mei, S. L. Shang, L. Q. Chen, Z. K. Liu, "A mixed-space approach to first-principles calculations of phonon frequencies for polar materials", J. Phys. Condens. Matter. 2010, 22, 202201.

[141] R. L. Puurunen, "Surface chemistry of atomic layer deposition: A case study for the trimethylaluminum/water process", J. Appl. Phys. 2005, 97, 121301.

[142] T. Aaltonen, M. Ritala, T. Sajavaara, J. Keinonen, M. Leskelä, "Atomic Layer Deposition of Platinum Thin Films", Chem. Mater. 2003, 15, 1924-1928.

[143] S. M. George, "Atomic Layer Deposition: An Overview", Chem. Rev. 2010, 110, $111-131$.

[144] J. A. Scheer, P. Wahlström, P. Wurz, "Scattering of light molecules from Al2O3 surfaces", Nucl. Instr. Meth. Phys. Res. B 2007, 256, 76-80.

[145] J. A. Scheer, P. Wahlström, P. Wurz, "Scattering of light molecules from thin Al2O3 films", Nucl. Instr. Meth. Phys. Res. B 2009, 267, 2571-2574.

[146] H. Lüth, Solid Surfaces, Interfaces and Thin Films, Vol. 5th, Springer, Berlin Heidelberg, 2010, p. 192.

[147] V. Cimalla, M. Baeumler, L. Kirste, M. Prescher, B. Christian, T. Passow, F. Benkhelifa, F. Bernhardt, G. Eichapfel, M. Himmerlich, S. Krischok, J. Pezoldt, "Densification of Thin Aluminum Oxide Films by Thermal Treatments", MSA 2014, 5, 628-638.

[148] G. Krautheim, T. Hecht, S. Jakschik, U. Schröder, W. Zahn, "Mechanical stress in ALD-Al2O3 films", Appl. Surf. Sci. 2005, 252, 200-204.

[149] H. Qin, P. Sutter, G. Zhou, N. Jacobson, "The Crystallization of Amorphous Aluminum Oxide Thin Films Grown on $\operatorname{NiAl}(100) "$, J. Am. Ceram. Soc. 2014, 97, $2762-2769$.

[150] S. M. Janke, M. Pavanello, G.-J. Kroes, D. Auerbach, A. M. Wodtke, A. Kandratsenka, "Toward Detection of Electron-Hole Pair Excitation in H-atom Collisions with $\mathrm{Au}(111)$ : Adiabatic Molecular Dynamics with a Semi-Empirical FullDimensional Potential Energy Surface", Z. Phys. Chem. 2013, 227, 1467-1490. 
[151] C. Steinsiek, P. Shirhatti, J. Geweke, C. Bartels, A. M. Wodtke, "Work Function Dependence of Vibrational Relaxation Probabilities: $\mathrm{No}(\mathrm{V}=2)$ Scattering from Ultra-Thin Metallic Films of $\mathrm{Ag} / \mathrm{Au}(111) "$, J. Phys. Chem. C 2018, 122, 10027-10033.

[152] Y. Wang, H. J. Qian, K. Morokuma, S. Irle, "Coupled cluster and density functional theory calculations of atomic hydrogen chemisorption on pyrene and coronene as model systems for graphene hydrogenation", J. Phys. Chem. A 2012, 116, 7154-60.

[153] X. Sha, B. Jackson, D. Lemoine, B. Lepetit, "Quantum studies of H atom trapping on a graphite surface", J. Chem. Phys. 2005, 122, 14709.

[154] E. R. M. Davidson, J. Klimeš, D. Alfè, A. Michaelides, "Cooperative Interplay of van der Waals Forces and Quantum Nuclear Effects on Adsorption: H at Graphene and at Coronene", ACS Nano 2014, 8, 9905-9913.

[155] E. Bahn, A. Tamtögl, J. Ellis, W. Allison, P. Fouquet, "Structure and dynamics investigations of a partially hydrogenated graphene/Ni(111) surface", Carbon 2017, $114,504-510$.

[156] M. Kammler, A. Kandratsenka, unpublished results.

[157] G. Kresse, J. Hafner, "Ab initiomolecular dynamics for liquid metals", Phys. Rev. $B$ 1993, 4\%, 558-561.

[158] G. Kresse, J. Hafner, "Ab initiomolecular-dynamics simulation of the liquidmetal-amorphous-semiconductor transition in germanium", Phys. Rev. B 1994, 49, 14251-14269.

[159] G. Kresse, J. Furthmüller, "Efficient iterative schemes for ab initio total-energy calculations using a plane-wave basis set", Phys. Rev. B 1996, 54, 11169-11186.

[160] G. Kresse, J. Furthmüller, "Efficiency of ab-initio total energy calculations for metals and semiconductors using a plane-wave basis set", Comput. Mater. Sci. 1996, 6, 15-50.

[161] P. E. Blöchl, "Projector augmented-wave method", Phys. Rev. B 1994, 50, 1795317979.

[162] G. Kresse, D. Joubert, "From ultrasoft pseudopotentials to the projector augmentedwave method", Phys. Rev. B 1999, 59, 1758-1775.

[163] O. K. Andersen, "Linear methods in band theory", Phys. Rev. B 1975, 12, 30603083. 
[164] D. W. Boukhvalov, M. I. Katsnelson, A. I. Lichtenstein, "Hydrogen on graphene: Electronic structure, total energy, structural distortions and magnetism from first-principles calculations", Phys. Rev. B 2008, 77, 035427.

[165] S. M. Janke, D. J. Auerbach, A. M. Wodtke, A. Kandratsenka, "An accurate fulldimensional potential energy surface for $\mathrm{H}-\mathrm{Au}(111)$ : Importance of nonadiabatic electronic excitation in energy transfer and adsorption", J. Chem. Phys. 2015, 143, 124708 . 


\section{Acknowledgement}

First of all, I would like to thank Prof. Dr. Alec M. Wodtke for giving me the opportunity to work in his group, for his support, motivation, brilliant ideas and suggestions that he provided for me during my PhD study.

Next, special thanks to Dr. Oliver Bünermann for all the help he provided throughout my whole $\mathrm{PhD}$ time. As the leader of my $\mathrm{PhD}$ project, he was always available for solving problems. I sincerely appreciate that.

I would also like to thank people who worked on the RAT machine, Dr. Hongyan Jiang, who finished his $\mathrm{PhD}$ thesis on the RAT machine in 2016. And all the Master and Bachelor students, who worked with me on several projects: Hansjochen Köckert, Nils Hertl, Kerstin Krüger and Patrick Floß. I also thank the theoreticians in the group, Dr. Alexander Kandratsenka, Dr. Svenja Janke and Marvin Kammler for their useful discussions, for comparing experimental data with computational results.

I would like to thank Prof. Dr. Dirk Schwarzer for all his support and for being the second member of my thesis committee.

I want to acknowledge Prof. Daniel J. Auerbach for his help in understanding surface scattering over the past years.

I would like to thank Prof. Dr. Xuming Yang and his hole working group for my research stay in China and their support in learning details about the Rydberg Atom Tagging technique. I specially appreciate Dr. Kaijun Yuan, Dr. Zhichao Chen, Shengrui Yu and $\mathrm{Su} \mathrm{Shu}$, for their great collaboration in the laboratory and for their support during my stay in China.

I also thank Prof. Dr. Jörg Behler, Prof. Dr. Jörg Schroeder, Prof. Dr. Jürgen Troe and PD Dr. Thomas Zeuch for being a member of my examination board.

My special thanks belong to all the people in the Wodtke research group and those associated with it for the warm and constructive atmosphere, for all their help, friendship and good company.

I further want to acknowledge the mechanical and electronic work shops at the Institute of Physical Chemistry and our mechanic meister within the workgroup Reinhard Bürsing for his technical support and fast developments. 
Last but not least to my family (especially my parents, my brother and sisters) I am ever so grateful for all the support and happiness they have given me and the faith they have in me.

My friends I would like to thank for all the good time we have spent together and for the loyal friendship they have shown. 